Using Ecological Niche Modelling to Predict Climate Change Responses of Ten Key Fishery Species in Aotearoa New Zealand

BY

\title{
Amber Brooks
}

A thesis submitted to Victoria University of Wellington in partial fulfilment of the requirements for the degree of Master of Science in Ecology and Biodiversity.

Victoria University of Wellington

2020 
This thesis was conducted under the supervision of:

\section{Dr. Peter Ritchie}

Victoria University of Wellington

And

Dr. Matt Dunn

National Institute of Water \& Atmospheric Research Ltd (NIWA) 


\section{Abstract}

The long-term sustainability and security of food sources for an increasing human population will become more challenging as climate change alters growing and harvesting conditions. Significant infrastructure changes could be required to continue to supply food from traditional sources. Fisheries remains the only major protein supply directly harvested from the wild. This likely makes it the most sensitive primary sector to climate change. Overfishing is an additional concern for harvested species. There is a need to anticipate how marine species may respond to climate change to help inform how management might best be prepared for shifting distributions and productivity levels. The most common response of mobile marine species to changes in climate is an alteration of their geographic distributions and/or range shifts. Predicting changes to a species' range could promote a timely development of more sustainable harvest strategies. Additionally, these predictions could reduce potential conflict when different management areas experience increasing or decreasing catches. Ecological Niche Modelling (ENM) is a helpful approach for predicting the response of key fishery species to climate change scenarios.

The overall aim of this research was to use the maximum entropy method, Maxent, to perform ENM on 10 commercially important fishery species, managed under the Quota management system in Aotearoa (New Zealand). Occurrence data from trawl surveys was used along with climate layers from Bio-ORACLE to estimate the species niche and then predict distributions in four different future climate scenarios, called Representative Concentration Pathway Scenarios (RCPS), in both 2050 and 2100. With little consensus over the best settings and way to apply the Maxent method, hundreds of variations were tried for each species, and the best model chosen from trial experimentation.

In general, Maxent performed well, with evaluation metrics for best models showing little omission error and good discriminatory ability. There was, however, considerable variation between the different species responses to the future climate scenarios. Consistent with other studies, species able to tolerate sub-tropical or temperate conditions tended to expand southward, while subantarctic species generally contracted within their preferred environment. The increasing emissions or 'business as usual' climate change scenario consistently presented the most extreme difference from modern predictions. Northern regions of prediction, where sub-tropical or temperate species increased in probability of presence, were often highly uncertain due to novel conditions in future environments. Southern regions were usually less uncertain. Surface temperature consistently influenced base models more so than any other covariates considered, with the exception of bathymetry. 
Some predictions showed common areas of relative stability, such as hoki and ling on the southern Chatham Rise, potentially indicating future refugia. The preservation of habitats in the putative refugia may be important for long-term fisheries resilience. Furthermore, most species that showed large predicted declines are currently heavily harvested and managed. Overfishing could compound the effects of climate change and put these fisheries at serious risk of collapse. Identification of potential refugial areas could aid strategy adjustments to fishing practise to help preserve stock viability. Additionally, when some species shift, there are areas where new fisheries may emerge.

This study offers a perspective of what future distributions could be like under different climate scenarios. The ENM predicts that the 'business as usual' scenario, where 'greenhouse gas' emissions continue to rise throughout the century, will have a negative impact on multiple aspects of distribution. However, in a reduced emissions scenario, less extreme range shifts are predicted. This study has provided a predictive approach to how fisheries in Aotearoa might change. The next step is to determine whether there is any evidence for the beginning of these changes and to consider how fisheries might best adapt. 


\section{Acknowledgements}

First and foremost, I'd like to thank both of my supervisors, Dr. Peter Ritchie and Dr. Matt Dunn, both of whom contributed insightful perspectives that shaped the way I thought about this project. Their encouragement, advice and feedback has been invaluable. Additionally, l'd like to thank those associated with collecting, collating and permitting the use of, the dataset used in this study, including Fisheries New Zealand, Ministry for Primary Industries, and the National Institute of Water and Atmospheric Research.

I would also like to thank the people I have met and worked alongside throughout the completion of this study, in particular everybody in Peter Ritchie's research group. Leah, Yvan, Tom and Alex, all made my time during study more enjoyable and often had helpful advice despite our different fields of research.

Finally, I'd like to thank my family and friends that have supported me over the last two years. For all the kind words and cooked meals that were shared with me, I am incredibly grateful. I owe special thanks to my mum, Ana Brooks, whose support was immensely helpful and greatly appreciated during the completion of this project. 


\section{Table of Contents}

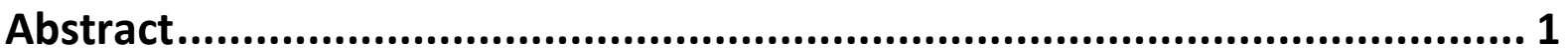

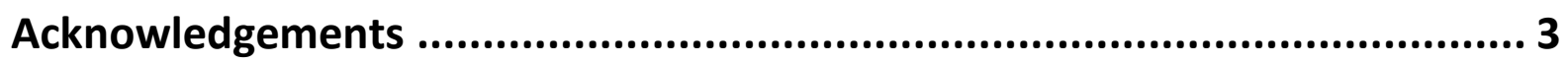

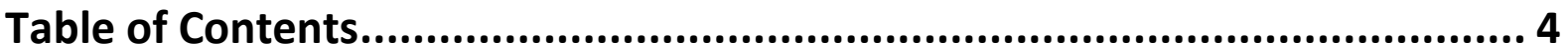

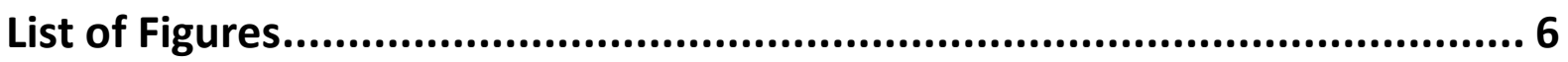

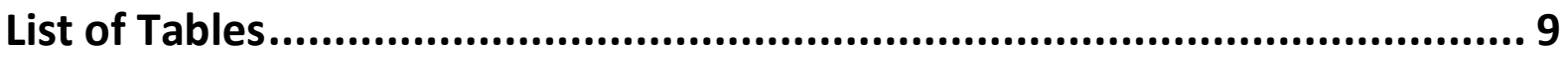

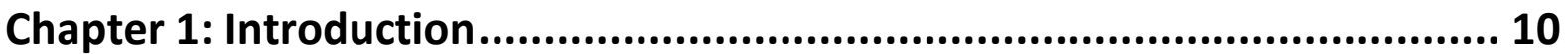

1.1: Climate Change Impacts on the Marine Environment..............................................10

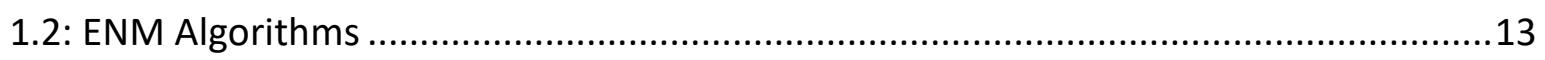

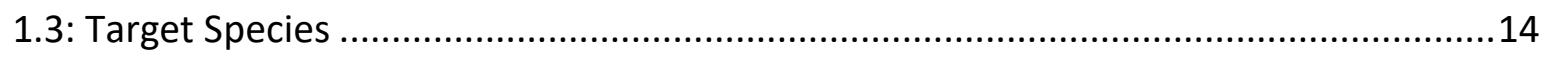

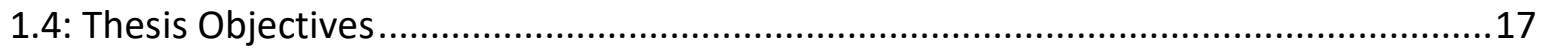

Chapter 2: Methods ................................................................................. 18

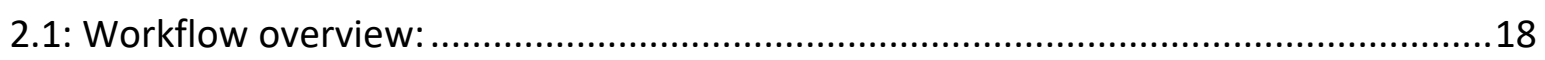

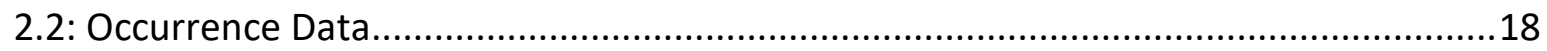

2.2.1: Preparing Occurrence Data ...................................................................... 18

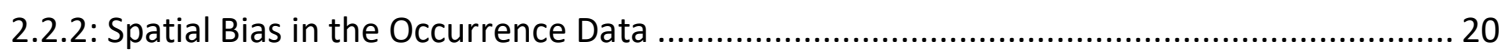

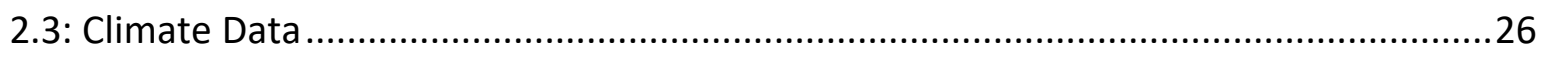

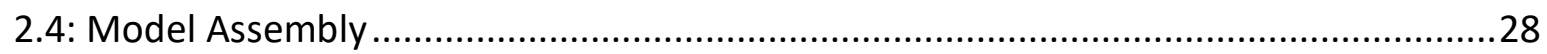

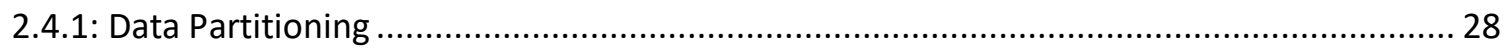

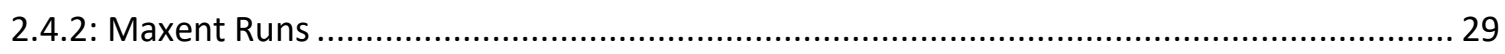

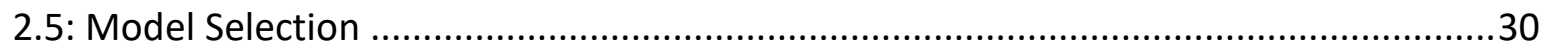

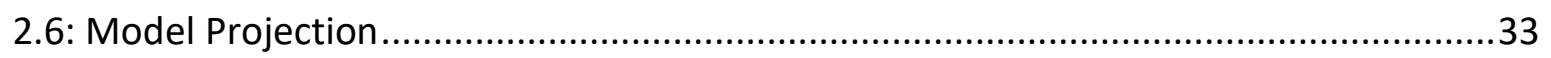

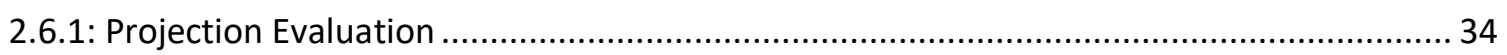

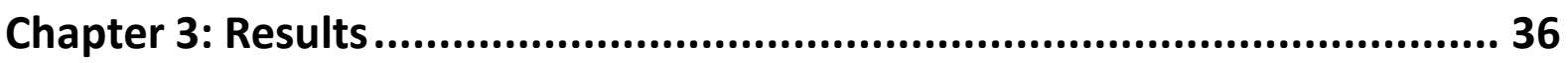

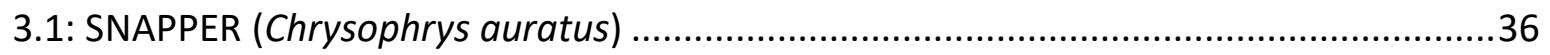

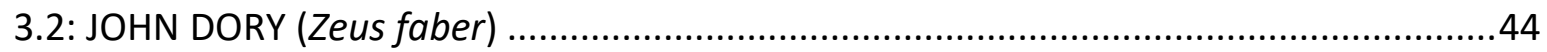

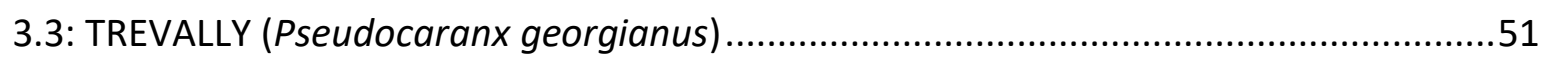

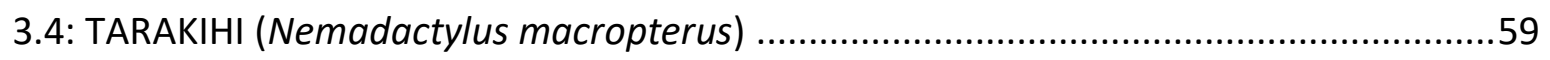

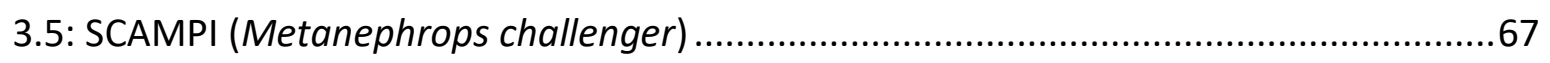

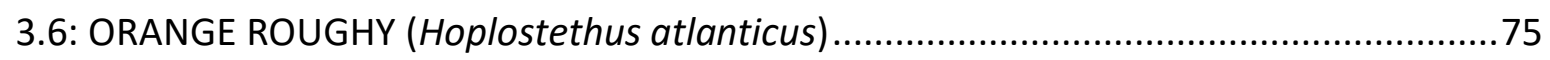

3.7 SOUTHERN BLUE WHITING (Micromesistius australis) ................................................8

3.8: HOKI (Macruronus novaezelandiae) ......................................................................91

3.9: LING (Genypterus blacodes) ..............................................................................99 
3.10: New Zealand ARROW SQUID (Nototodarus sloanii) ...........................................107

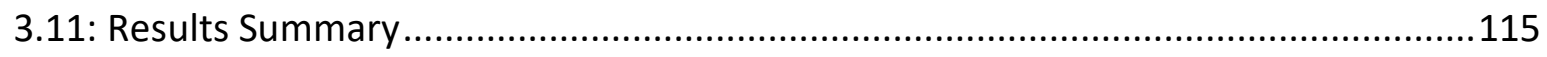

Chapter 4: Discussion ...............................................................120

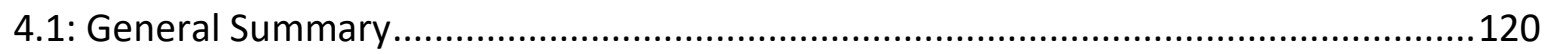

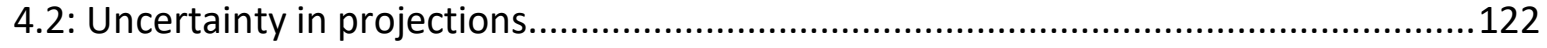

4.2.1: Incomplete and implausible temperature responses.................................................. 122

4.2.2: Novel climates due to variables with values outside of the training range ..................... 125

4.3: Implications of Different Climate Scenarios..........................................................127

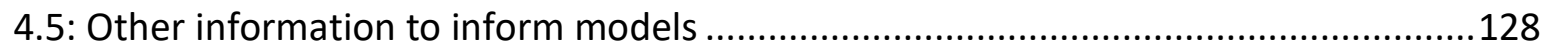

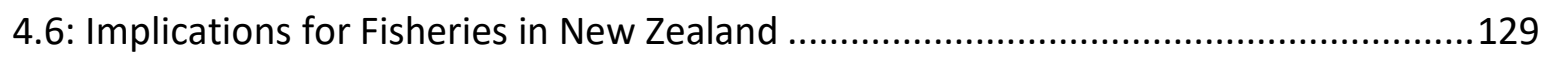

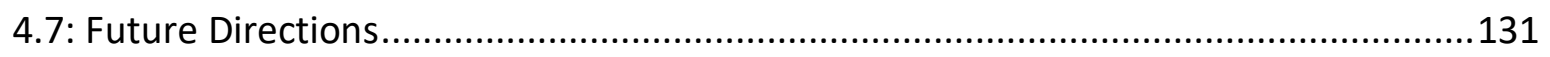

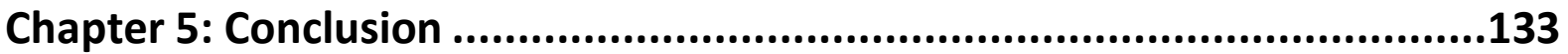

References.................................................................................134

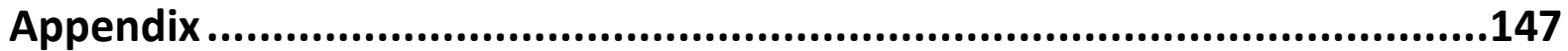




\section{List of Figures}

Figure 2.1: Overview of the methods

Figure 2.2: Total geographic coverage of the occurrence data set

Figure 2.3: Total number of records in the occurrence data set per year caught

Figure 2.4: Maximum depth of records $(A)$ and minimum depth of records $(B)$ in the total occurrence data set.

Figure 2.5: Example of the different background adjustment methods used in this study

Figure 3.1.1: Snapper; Six best Maxent models projected on modern climate layers

Figure 3.1.2: Snapper; Recorded modern distribution and probability distribution projections onto the modern climate and each of the four climate scenarios RCP 2.6, 4.5, 6.0 and 8.5 in 2050 and 2100

Figure 3.1.3: Snapper; MESS Maps of RCPS 2.6 in 2050 and RCPS 8.5 in 2100. MESS maps for all climate scenarios can be found in Appendix D.1

Figure 3.1.4: Snapper; Predictor response curves indicating how variables used for training affected the base model

Figure 3.1.5: Snapper; Predictor variable contribution to the base model

Figure 3.1.6: Snapper; Limiting factors plots of Modern RCPS 8.5 in 2100 climates

Figure 3.2.1: John dory; Six best Maxent models projected on modern climate layers

Figure 3.2.2: John dory; Recorded modern distribution and probability distribution projections onto the modern climate and each of the four climate scenarios RCP 2.6, 4.5, 6.0 and 8.5 in 2050 and 2100

Figure 3.2.3: John dory; MESS Maps of RCPS 2.6 in 2050 and RCPS 8.5 in 2100. MESS maps for all climate scenarios can be found in Appendix D.2

Figure 3.2.4: John dory; Predictor response curves indicating how variables used for training affected the base model

Figure 3.2.5: John dory; Predictor variable contribution to the base model

Figure 3.2.6: John dory; Limiting factors plots of Modern RCPS 8.5 in 2100 climates

Figure 3.3.1: Trevally; Six best models projected on modern climate layers

Figure 3.3.2: Trevally; Recorded modern distribution and probability distribution projections onto the modern climate and each of the four climate scenarios RCP 2.6, 4.5, 6.0 and 8.5 in 2050 and 2100

Figure 3.3.3: Trevally; MESS Maps of RCPS 2.6 in 2050 and RCPS 8.5 in 2100. MESS maps for all climate scenarios can be found in Appendix D.3

Figure 3.3.4: Trevally; Predictor response curves indicating how variables used for training affected the base model

Figure 3.3.5: Trevally; Predictor variable contribution to the base model 
Figure 3.3.6: Trevally; Limiting factors plots of Modern RCPS 8.5 in 2100 climates

Figure 3.4.1: Tarakihi; Six best Maxent models projected on modern climate layers

Figure 3.4.2: Tarakihi; Recorded modern distribution and probability distribution projections onto the modern climate and each of the four climate scenarios RCP 2.6, 4.5, 6.0 and 8.5 in 2050 and 2100

Figure 3.4.3: Tarakihi; MESS Maps of RCPS 2.6 in 2050 and RCPS 8.5 in 2100 . MESS maps for all climate scenarios can be found in Appendix D.4

Figure 3.4.4: Tarakihi; Predictor response curves indicating how variables used for training affected the base model.

Figure 3.4.5: Tarakihi; Predictor variable contribution to the base model

Figure 3.4.6: Tarakihi; Limiting factors plots of Modern RCPS 8.5 in 2100 climates.

Figure 3.5.1: Scampi; Six best Maxent models projected on modern climate layers

Figure 3.5.2: Scampi; Recorded modern distribution and probability distribution projections onto the modern climate and each of the four climate scenarios RCP 2.6, 4.5, 6.0 and 8.5 in 2050 and 2100

Figure 3.5.3: Scampi; MESS Maps of RCPS 2.6 in 2050 and RCPS 8.5 in 2100. MESS maps for all climate scenarios can be found in Appendix D.5

Figure 3.5.4: Scampi; Predictor response curves indicating how variables used for training affected the base model.

Figure 3.5.5: Scampi; Predictor variable contribution to the base model

Figure 3.5.6: Scampi; Limiting factors plots of Modern RCPS 8.5 in 2100 climates

Figure 3.6.1: Orange roughy; Six best Maxent models projected on modern climate layers

Figure 3.6.2: Orange roughy; Recorded modern distribution and probability distribution projections onto the modern climate and each of the four climate scenarios RCP 2.6, 4.5, 6.0 and 8.5 in 2050 and 2100

Figure 3.6.3: Orange roughy; MESS Maps of RCPS 2.6 in 2050 and RCPS 8.5 in 2100. MESS maps for all climate scenarios can be found in Appendix D.6

Figure 3.6.4: Orange roughy; Predictor response curves indicating how variables used for training affected the base model.

Figure 3.6.5: Orange roughy; Predictor variable contribution to the base model

Figure 3.6.6: Orange roughy; Limiting factors plots of Modern RCPS 8.5 in 2100 climates

Figure 3.7.1: Southern blue whiting; Six best Maxent models projected on modern climate layers

Figure 3.7.2: Southern blue whiting; Recorded modern distribution and probability distribution projections onto the modern climate and each of the four climate scenarios RCP 2.6, 4.5, 6.0 and 8.5 in 2050 and 2100

Figure 3.7.3: Southern blue whiting; MESS Maps of RCPS 2.6 in 2050 and RCPS 8.5 in 2100 . MESS maps for all climate scenarios can be found in Appendix D.7 
Figure 3.7.4: Southern blue whiting; Predictor response curves indicating how variables used for training affected the base model.

Figure 3.7.5: Southern blue whiting; Predictor variable contribution to the base model

Figure 3.7.6: Southern blue whiting; Limiting factors plots of Modern RCPS 8.5 in 2100 climates

Figure 3.8.1: Hoki; Six best Maxent models projected on modern climate layers

Figure 3.8.2: Hoki; Recorded modern distribution and probability distribution projections onto the modern climate and each of the four climate scenarios RCP 2.6, 4.5, 6.0 and 8.5 in 2050 and 2100

Figure 3.8.3: Hoki; MESS Maps of RCPS 2.6 in 2050 and RCPS 8.5 in 2100 . MESS maps for all climate scenarios can be found in Appendix D.8

Figure 3.8.4: Hoki; Predictor response curves indicating how variables used for training affected the base model.

Figure 3.8.5: Hoki; Predictor variable contribution to the base model

Figure 3.8.6: Hoki; Limiting factors plots of Modern RCPS 8.5 in 2100 climates

Figure 3.9.1: Ling; Six best Maxent models projected on modern climate layers

Figure 3.9.2: Ling; Recorded modern distribution and probability distribution projections onto the modern climate and each of the four climate scenarios RCP 2.6, 4.5, 6.0 and 8.5 in 2050 and 2100

Figure 3.9.3: Ling; MESS Maps of RCPS 2.6 in 2050 and RCPS 8.5 in 2100 . MESS maps for all climate scenarios can be found in Appendix D.9

Figure 3.9.4: Ling; Predictor response curves indicating how variables used for training affected the base model.

Figure 3.9.5: Ling; Predictor variable contribution to the base model

Figure 3.9.6: Ling; Limiting factors plots of Modern RCPS 8.5 in 2100 climates

Figure 3.10.1: Arrow squid; Six best Maxent models projected on modern climate layers

Figure 3.10.2: New Zealand arrow squid; Recorded modern distribution and probability distribution projections onto the modern climate and each of the four climate scenarios RCP 2.6, 4.5, 6.0 and 8.5 in 2050 and 2100

Figure 3.10.3: Arrow squid; MESS Maps of RCPS 2.6 in 2050 and RCPS 8.5 in 2100. MESS maps for all climate scenarios can be found in Appendix D.10

Figure 3.10.4: Arrow squid; Predictor response curves indicating how variables used for training affected the base model.

Figure 3.10.5: Arrow squid; Predictor variable contribution to the base model

Figure 3.10.6: Arrow squid; Limiting factors plots of Modern RCPS 8.5 in 2100 climates

Figure 3.11.1: Percentage change in probability of presence from modern projections, according to three different thresholds of habitat preference, for each climate scenario in 2050 and 2100 


\section{List of Tables}

Table 2.1: Number of records for each target species before and after rarefication

Table 2.2: Trail codes for Maxent runs showing the combinations methods used

Table 3.1.1: Snapper; ENMeval evaluation metrics of six best models

Table 3.1.2: Snapper; Change in probability of presence values compared to the modern projection for each of the four climate scenarios 2050 and 2100

Table 3.2.1: John dory; ENMeval evaluation metrics of six best models

Table 3.2.2: John dory; Change in probability of presence values compared to the modern projection for each of the four climate scenarios 2050 and 2100

Table 3.3.1: Trevally; ENMeval evaluation metrics of six best models

Table 3.3.2: Trevally; Change in probability of presence values compared to the modern projection for each of the four climate scenarios 2050 and 2100

Table 3.4.1: Tarakihi; ENMeval evaluation metrics of six best models

Table 3.4.2: Tarakihi; Change in probability of presence values compared to the modern projection for each of the four climate scenarios 2050 and 2100

Table 3.5.1: Scampi; ENMeval evaluation metrics of six best models

Table 3.5.2: Scampi; Change in probability of presence values compared to the modern projection for each of the four climate scenarios 2050 and 2100

Table 3.6.1: Orange roughy; ENMeval evaluation metrics of six best models.

Table 3.6.2: Orange roughy; Change in probability of presence values compared to the modern projection for each of the four climate scenarios 2050 and 2100

Table 3.7.1: Southern blue whiting; ENMeval evaluation metrics of six best models

Table 3.7.2: Southern blue whiting; Change in probability of presence values compared to the modern projection for each of the four climate scenarios 2050 and 2100

Table 3.8.1: Hoki; ENMeval evaluation metrics of six best models

Table 3.8.2: Hoki; Change in probability of presence values compared to the modern projection for each of the four climate scenarios 2050 and 2100

Table 3.9.1: Ling; ENMeval evaluation metrics of six best models

Table 3.9.2: Ling; Change in probability of presence values compared to the modern projection for each of the four climate scenarios 2050 and 2100

Table 3.10.1: Arrow squid; ENMeval evaluation metrics of six best models

Table 3.10.2: Arrow squid; Change in probability of presence values compared to the modern projection for each of the four climate scenarios 2050 and 2100 


\section{Chapter 1: Introduction}

\section{1: Climate Change Impacts on the Marine Environment}

Anthropogenic climate change has become the forefront of most research globally, is topical in mainstream media, and is the source of much political debate. Anticipating changes in global climate and impacts may better enable society to prepare and understand what will be required to adapt. Discussions of climate change impacts have been disproportionately focussed on terrestrial ecosystems and species, while impacts on the marine environment has often been under-reported (Turra et al., 2016; Donelson et al., 2019; Frost et al., 2017; Robinson et al., 2011). Recently however, concerns have been raised in mainstream media reports about the effects of global climate change on marine environments (Beck, 2018; May, 2019; Neilson, 2019; Stevens \& Noll, 2019) and a 2019 study by Cheng et al. (2019) suggested that the marine environment is being impacted to a larger extent than was previously expected. Impacts on the marine environment include, but are not limited to, rapid ocean warming, rising sea levels, ocean acidification, declining oxygen levels, and primary productivity shifts (Cheng et al., 2019; Free et al., 2019, Law et al., 2017). There is an urgent need to understand the implications of these changes on marine species ecosystems, especially those that support valuable fisheries.

Species that cannot tolerate changes to their environmental conditions usually respond by shifting their geographic range, including withdrawal to areas of refugia, and/or adapt to the altered conditions (Donelson et al., 2019; Nogués-Bravo et al., 2018). In the absence of these types of responses, extirpation or extinction of species or populations may occur (Nogués-Bravo et al., 2018). Phenotypic plasticity or altering phenology may permit species to temporarily persist in an area and avoid moving or adapting (Crozier \& Hutchings, 2014). However, persistence through extreme environmental change will likely require more permanent adjustment and/or a combination of these responses (Crozier \& Hutchings, 2014; Donelson et al., 2019). The current rate of the changing climate is not conducive to adaption unless rapid, a possibility reserved for species with high reproductive rates and short generation times or ' $r$-selected' life histories (Colautti \& Barrett, 2013; Crozier \& Hutchings, 2014; Reznick \& Ghalambor, 2001). This, and the mobile nature of most marine organisms, often undertaking long distance migrations or being dispersed by pelagic larvae in ocean currents, suggests distribution shifts will more frequently be observed as responses of marine species to climate change (Donelson et al., 2019; Hiddink \& Ter Hofstede, 2008).

Many marine species' distributions are dynamic and strongly linked to temperature preferences (Cheung et al., 2012; Sunday et al., 2012). Although ocean warming will have varied effects on different marine taxa (Free et al., 2019), latitudinal and depth range shifts in response to ocean 
warming have already been observed numerous times (Cheung et al., 2012; Dambach \& Rödder, 2011; Jung et al., 2014; Morley et al., 2018; Perry et al., 2005). Mostly poleward shifts have been observed and are expected to increase, with species in sup-polar regions at high risk of extinction due to invasion and competition from species currently in temperate and tropical regions (Cheung et al., 2009; Cheung et al., 2013; Morley et al., 2018). In this case, this pattern would result in an increasing dominance of warm water species, a phenomenon called "tropicalisation" (Cheung et al., 2012; Cheung et al., 2013). These large scale redistributions are likely in response to physiological stress or change in food availability due to higher temperatures (Plagányi, 2019). Other factors associated with ocean warming, such as ocean acidification and reduced oxygen concentration, are expected to compound warming effects (Hofmann \& Schellnhuber, 2009). The resulting changes in species composition and richness will likely substantially impact fisheries worldwide (Cheung et al., 2009; Dambach \& Rödder, 2011). As species shift across current management boundaries, conflicts and management disputes may arise over catch allocations (Bell et al., 2014; Miller \& Munro, 2004). Furthermore overfishing of shifted populations would further compromise the resilience of species already contracting in response to warmer temperatures (Free et al., 2019).

Global productivity of marine fishes has declined and is expected to continue to do so as a result of climate change and other anthropogenic factors (Free et al., 2019). However, changes in productivity are expected to vary in different regions (Blanchard et al., 2012; Cheung et al., 2016; Moore et al., 2018). Decline of fisheries productivity coinciding with human population increases will almost certainly reduce per-person seafood availability (Plagányi, 2019). In future, suitable responses to redistribution of fisheries worldwide could alleviate some pressure on fisheries. Implementation of effective management strategies and adaptation requires better understanding of fisheries' responses to climate change (Cheung et al., 2013). Anticipating redistribution of key fishery species is a first step in achieving this. Tools such as Ecological niche modelling (ENM) may allow better anticipation of species shifts in response to climate change. ENM has the potential to aid development of sustainable management practices in response to changing climates.

ENM or Species Distribution Modelling (SDM)* allows exploration of possible range shifts and species overlaps by predicting species' distributions based on correlations between environmental covariates and species' occurrences. ENM has also been used to aid species delimitation (Raxworthy et al., 2007), invasive species studies (Thuiller et al., 2005), conservation planning (Lindsay et al., 2016; Moore et al., 2016), phylogenetics (Graham et al., 2004) and population genetics studies (Mestre et al., 2015; McCallum et al., 2014). See Graham et al. (2006) and Martinez-Meyer et al. (2004) for other purposes. Increased use of ENM to predict species' responses to future environments has coincided with increased data accessibility, technological advancements and rising 
concerns about climate change. Unfortunately, far fewer marine ENM studies have been done than terrestrial studies (Robinson et al., 2011), and in 2017 only 17\% of these had been done specifically as climate change investigations (Robinson et al., 2017). ENM as a tool to predict responses to climate change in the marine realm is an underutilised resource.

Lack of marine ENM studies could be attributed to the difficulty in collecting occurrence data compared to terrestrial environments (Kaschner et al., 2006; Mannocci et al., 2018; Tyberghein et al., 2012). Challenges in the marine environment that restrict collection, such as clarity and depth, have resulted in marine surveys historically falling behind the terrestrial ones (Costello et al., 2010; Zhang \& Vincent, 2017). Most marine ENM studies have been performed in temperate northern regions, particularly the Northern Atlantic where survey effort is substantial (Breece et al., 2016; Bruge et al. 2016; Robinson et al., 2017). There has been a lack of ENM studies in other regions with high survey effort for fisheries monitoring, such as South Africa and parts of South America (Robinson et al., 2017). Additionally, in regions likely to be substantially affected by potential poleward shifts, such as tropical waters around Indonesia and Africa, ENM has rarely been utilised (Barros et al., 2014). This is likely due to wealth and institute density as well as lower survey effort. Furthermore, few marine ENM have been done specific to the Arctic, despite predicted substantial community composition changes species (Cheung et al., 2009; Cheung et al., 2013; Morley et al., 2018). In 2017 only 10\% of all marine ENM studies had been in Australasia and only 4 out of 236 marine ENM studies done worldwide were specific to New Zealand. None of the New Zealand studies were investigations into climate change responses (Robinson et al., 2017).

Further reservations to apply ENM to marine environments may be due to lack of fossil records or genetic data from the marine environment, which are often used in terrestrial studies to corroborate predictions (Gavin et al., 2014). However, marine environments are more mobile than the terrestrial, from tides to ocean currents, and therefore supports greater potential for long distance dispersal (Carr et al., 2003; L. Robinson et al., 2011). Most marine organisms generally disperse farther and faster than terrestrial species and are more likely to occupy a greater amount of the area available with suitable habitat (Donelson et al., 2019; L. Robinson et al., 2011). As a result, ENM may be expected to predict marine species' future distributions better than those of terrestrial organisms. Additionally, marine environmental data and can be obtained from broad-scale remotely sensed or modelled data sources, a benefit not as applicable for terrestrial studies, which are required frequently to include microclimate information (Robinson et al., 2011). 
*The terms ENM and SDM model are often used interchangeably. What the distinction is and whether it is important appears an unresolved topic of debate (Peterson 2006; Peterson 2012; Elith \& Leathwick 2009; Franklin 2010; Sillero 2011; Araújo \& Peterson 2012; Warren 2012). Although the aim of this study is not to characterise the ecological niche but to estimate the potential distributions, doing so requires niche estimates based on a set of variables (Peterson, 2012). Furthermore, interpreting how environmental changes may elicit a species' response and influence distributions relies on niche theory because it assumes a correlation between the variables used and species' potential geographic distributions (Peterson, 2012, Wiens et al., 2009). Thus, the phrase ENM is used rather than SDM in this thesis.

\section{2: ENM Algorithms}

A wide variety of ENM algorithms are now available and easily accessible. These include algorithms that use Presence Absence (PA) data such as generalised linear models (Nelder \& Wedderburn, 1972; Zuur et al., 2010), generalised additive models (Zuur et al., 2010) and artificial neural networks (D’heygere et al., 2006; Fukuda et al., 2013). Others are able to utilise Presence Only (PO) data such as BIOCLIM (Booth 1985; Booth et al., 2014; Parra et al., 2004), DOMAIN (Carpenter et al., 1993; Segurado \& Araujo, 2004), Maxent (Phillips et al., 2006; Phillips et al., 2004), and GARP (Anderson, 2003; Peterson, 2001). PA data contains both species presence and absence information and is usually collected in a systematic and targeted manner. PO data does not contain absence information and is usually more inconsistent and spatially biased than PA data. PO data however is more easily collected and is readily available from museums, herbariums, and online sources like the Global Biodiversity Information Facility (GBIF; http://www.gbif.org) dataset (Elith et al., 2006). Algorithms that utilise PO data are therefore more easily utilised and PO data is a valuable resource, given the challenges of using it are overcome (Elith et al., 2006).

In 2017, the most used ENM software was Maxent, the popularity of which has substantially increased since its introduction in 2006 (Gobeyn et al., 2019; Morales et al., 2017; Phillips et al., 2006). Maxent is a maximum entropy based machine learning algorithm that uses presence and background data to predict the probability distribution of a species based on a given set of environmental variables (Manzoor et al., 2018). Although less mature, Maxent has often performed well compared with other ENM methods (Derville et al., 2018; Elith \& Graham, 2009; Elith et al., 2006; Phillips et al., 2006; Ray et al., 2018; Shabani et al., 2016; Tarkesh \& Jetschke, 2012) particularly on small sample sizes (Papeş \& Gaubert, 2007; Pearson et al., 2007). However, there has been particular contention around whether Maxent or GARP (Genetic Algorithm for Rule-set Prediction) is superior (Chikerema et al., 2017; Peterson et al., 2007; Ray et al., 2018; Terribile \& Diniz-Filho, 2010). Most studies seem to favour Maxent and claim it achieves better predictions 
(Elith \& Graham, 2009; Phillips et al., 2006; Ray et al., 2018; Tarkesh \& Jetschke, 2012). Those that favour GARP either only use AUC statistics to evaluate models (Terribile \& Diniz-Filho, 2010), an evaluation metric that has been criticised when used exclusively (Lobo et al., 2008; Peterson et al., 2008), or received criticism for incorrect use of the Maxent (Peterson et al., 2007; Phillips, 2008). This was usually related to background data use, insufficient replicates or failing to utilise the flexibility of Maxent by relying on default settings (Anderson, 2015; Anderson \& Gonzalez, 2011; Peterson et al., 2007; Phillips, 2008). Studies favouring Maxent often explored more settings and features of the software (Elith \& Graham, 2009). Without considering key settings that can be altered to suit the data, the utility of Maxent is often underestimated (Anderson \& Gonzalez, 2011; Chikerema et al., 2017). Furthermore, ENM studies in general rarely attempt to estimate uncertainty in their predictions, reducing the ability to identify methods or models that produce more or less robust predictions (Cheung et al., 2016; Morley et al., 2018; Planque et al., 2011).

\section{3: Target Species}

This thesis considers a range of species managed under the New Zealand Quota Management System (QMS), in New Zealand (New Zealand) for which their occurrence records are regularly collected during fisheries research trawl surveys by the National Institute of Water and Atmosphere Research Ltd. (NIWA). These species were chosen due to their prevalence in literature, varied life histories and other biological traits, and diverse temperature and depth preferences, so as to have a range of species to compare and contrast. Target species include inshore species; snapper, john dory, trevally and tarakihi usually found at depths of less than $200 \mathrm{~m}$, and a number of deep-water species such as scampi, orange roughy, hoki, ling, southern blue whiting, and a species of New Zealand arrow squid. Each of these species are recognised as important fisheries in New Zealand and many have recreational and/or cultural value (Fisheries New Zealand, 2018). Species are listed with their most commonly used name, Māori and scientific names respectively. Where the common and Māori names were the same only this was used.

\section{Snapper or tāmure (Chrysophrys auratus Forster, 1801)}

Snapper are in the Sparidae family, made up of 38 Genera, and 159 species, distributed throughout tropical and temperate Atlantic, Indian, and Pacific Oceans (Paul, 1986). Snapper are one of the most commercially valuable inshore species in New Zealand, distributed mainly from the top of $\mathrm{NI}$ to top of SI (Parsons et al., 2014). Snapper are mostly prevalent in warm waters, and appear to have increased growth, survival and recruitment success in warmer waters (Fielder et al., 2005; Francis, 1993; Parsons et al., 2014). The same species is also found around coastal Australia and some Pacific Islands (Ashton et al., 2019; Sumpton et al., 2008). They are demersal fish with a depth range down 
to $200 \mathrm{~m}$ but are typically present in $15-60 \mathrm{~m}$ (Parsons et al., 2014; Smith et al., 1978). Snapper are relatively slow growing and long lived, reaching maturity at around 3-4 years and may live for up to 60 years (Parsons et al., 2014).

\section{Hoki (Macruronus novaezelandiae Hector, 1871)}

Hoki are in the Merlucciidae family, made up of 24 species and 5 Genera, distributed throughout the Atlantic, eastern Pacific, Tasmania and New Zealand, often in sub-antarctic waters (Alyling \& Cox, 1982). As an abundant commercial finfish species in New Zealand waters, hoki is New Zealand's largest fishery and is exported all over the world (Dunford et al., 2015; McKenzie, 2017). Hoki are widely distributed throughout New Zealand Exclusive Economic Zone at depths from 50 - 1000m, although they are typically caught between $400-600 \mathrm{~m}$ in southern regions, preferring cooler water temperatures (Hamer et al., 2012). Hoki are relatively fast-growing, reaching maturity at 3-5 years and living for up to 25 years (McKenzie, 2017).

\section{Orange roughy or nihorota (Hoplostethus atlanticus Collett, 1889)}

Orange roughy are part of the Trachichthyidae family, consisting of 49 species and 8 genera distributed in temperate oceans worldwide (Tingley \& Dunn, 2018). Orange roughy are widely distributed globally but absent from northern Indian and Pacific Oceans (Branch, 2001; Kulka et al., 2003; Laptikhovsky, 2008; Roberts et al., 2015; Varela et al., 2013). In New Zealand they are widespread from depths of 450-1800m, although usually caught between 700-1300m (Branch, 2001). Orange roughy are slow growing and exceptionally long lived, known to live well over 100 years (Andrews \& Tracey, 2003; Andrews et al., 2009; Tingley \& Dunn, 2018). Orange roughy don't tend to reach maturity until 30-40 years, and have low fecundity (Branch, 2001; Tingley \& Dunn, 2018).

\section{Tarakihi (Nemadactylus macropterus Forster, 1801)}

Tarakihi are part of the Cheilodactylidae family, with 27 species and 4 genera, distributed in subtropical and temperate waters both northern and southern hemispheres (Roberts et al., 2015). Tarakihi are widespread and commercially important marine fish in New Zealand and southern Australia (Burridge \& Smolenski, 2003). They are typically found on the continental shelf at depths of 80-100m but known depth range is from 10 up to 500m (Beentjes, 2011; Burridge \& Smolenski, 2003; McKenzie et al., 2017). Tarakihi have high fecundity, are relatively fast-growing, mature at 4-6 years and live upwards of 35 years (Burridge \& Smolenski, 2003; McKenzie et al., 2017).

Trevally or araara (Pseudocaranx georgianus Cuvier, 1833) 
Trevally are part of the Carangidae family, with 146 species and 30 genera, distributed in tropical waters in the Atlantic, Indian, and Pacific oceans (Roberts et al., 2015). Trevally are one of New Zealand's most important commercial inshore fish species (Fisheries New Zealand, 2018). Found in northern New Zealand and other areas throughout the world, although there is much confusion and inconsistency where naming of this species is concerned throughout the world (Fisheries New Zealand, 2018). Trevally are most common at depths of approximately $80 \mathrm{~m}$, although their depth range is thought to be $10-238 \mathrm{~m}$ (Mundy, 2005). Trevally can reach over 40 years of age (Fisheries New Zealand, 2018).

\section{Scampi or kōurarangi (Metanephrops challenger Balss, 1914)}

Scapi are part of the Nephropidae family, with 18 genera and 118 species distributed throughout both southern and northern hemispheres (Bell et al., 2013; Tshudy, 2003). In New Zealand, scampi are of high value, particularly as an exported species (Tuck et al., 2015; Van der Reis et al., 2018). They are generally found at depths of $200-600 \mathrm{~m}$ on muddy areas of the continental slope around eastern and western New Zealand, including the Chatham Islands (Bell et al., 2013; Major \& Jeffs, 2018; Tshudy, 2003; Tuck et al., 2015). Scampi can live for up to 15 years, reaching maturity at 3-4 years (Cryer \& Oliver, 2001), and are known to have low fecundity (Phillips, 2008).

\section{John dory or kuparu (Zeus faber Linnaeus, 1758)}

John dory are part of the Zeidae family, with 6 species and 2 genera, distributed in the Atlantic, Indian, and Pacific Oceans (Heemstra, 1980). John dory is distributed widely worldwide, usually found less than 200 m deep (Maravelias et al., 2007; Radford et al., 2018). In New Zealand they are distributed mainly around the NI and northern SI (Dunn \& Jones, 2013). John dory have been found to live up to eight years, in New Zealand (Caton \& McLoughlin, 2000) and mature at 4-5 years (Ismen et al., 2013).

\section{Southern blue whiting (Micromesistius australis Norman, 1937)}

Southern blue whiting are in the family Gadidae, which has 23 species and 13 genera distributed in sub-antarctic water in the Arctic, Atlantic and Pacific oceans at depths of 0-800m (Alyling \& Cox, 1982). Southern blue whiting occur in sub-antarctic waters off South America and southeast of New Zealand (Hanchet, 1999; O'Driscoll et al., 2016). New Zealand southern blue whiting are a major fishery with substantial annual landings (Fisheries New Zealand, 2018; O'Driscoll et al., 2016). Southern blue whiting are known to aggregate at 200-500m (O'Driscoll et al., 2016). They usually live to around 15 years but have been found to live up to 25 , and mature between 2-4 years (Fisheries New Zealand, 2018). 
Ling are part of the family Ophidiidae, with 258 species and 50 genera, found in the Atlantic, Indian and Pacific oceans (Roberts, 2015). Ling are found in depths of $100-800 \mathrm{~m}$ in the southern Pacific and Atlantic oceans (Dunn et al., 2010). The maximum recorded age for ling in New Zealand is 46 although they don't often live over 30 years (Dunn et al., 2010; Horn, 2005) and have relatively low fecundity compared to other deep water species (Paredes \& Bravo, 2005). Hoki, arrow squid and scampi are common components of their diet in New Zealand (Fisheries New Zealand, 2018).

\section{New Zealand arrow squid or wheketere (Nototodarus sloanii Gray, 1849)}

Arrow squid are part of the family Ommastrephidae, with 11 Genera and 21 species, found in all oceans of the world (Jereb \& Roper, 2005; Roper et al., 2010). N. sloani is endemic to New Zealand and found south of the convergence zone up to 600m depth (Fisheries New Zealand, 2018). Juveniles are found in shallower waters of $<200 \mathrm{~m}$ (Dunn, 2009). They live to around 1 year, have rapid growth and mature around 200 days (Dunn, 2009). There is a second closely related species, $N$. gouldi, which closely resembles N.solani, making it difficult to differentiate (Fisheries New Zealand, 2018). N. gouldi is generally found further north and on the west coast (Fisheries New Zealand, 2018). Although only N. sloanii was modelled in this thesis, discussion of how mistaken identification between these two species may have affected results has been included.

\section{4: Thesis Objectives}

The overall objective of this study is to establish a better understanding of how some New Zealand marine species included in the New Zealand Quota Management System (QMS) may respond to future climate change. The specific aims of this thesis were as follows:

- To appropriately collate and examine marine climate and fish occurrence data for use with the Maxent modelling method.

- To perform ENM on each target species under different parameters in order to find a model that best represented the ecological niche of the target species.

- To project best models for each species onto four different future climate scenarios (Representative Concentration Pathway Scenarios) in order to visualise their response.

- To consider the validity and reliability of the future predictions. 


\section{Chapter 2: Methods}

\section{1: Workflow overview:}

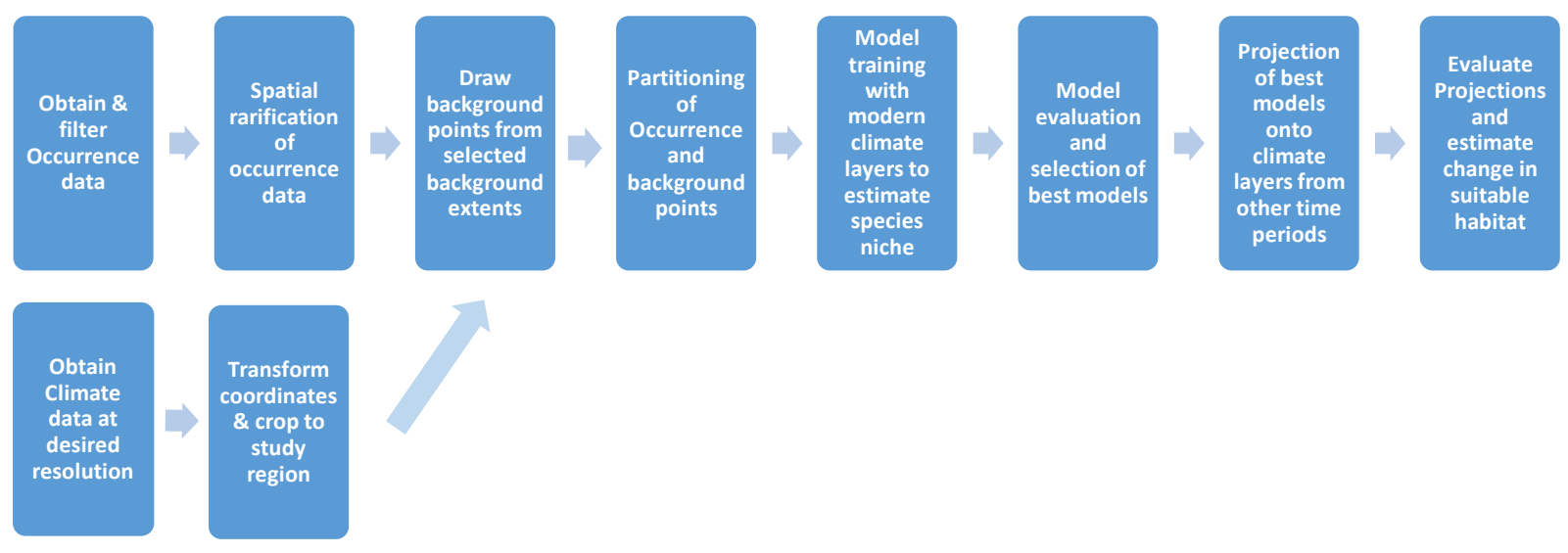

Figure 2.1: Overview of the methods

\section{2: Occurrence Data}

\subsection{1: Preparing Occurrence Data}

Occurrence data for New Zealand marine fishery species were obtained from the fisheries New Zealand database, which holds operational details, catch, and biological sampling data for all government-commissioned, research trawl surveys. Summary of the major survey series within this dataset can be found in Beentjes and Stevenson (2008); Dunn, Rickard, Sutton, and Doonan (2009); Morrison, Stevenson, and Hanchet (2001); O'Driscoll, MacGibbon, Fu, Lyon, and Stevens (2011); and Stevenson and MacGibbon (2018). These data were provided by NIWA (M. Dunn). The total geographical coverage of this dataset is presented in Figure 2.2. This dataset contained 660,905 catch records of 1,892 different marine species caught between 1960 and 2017, although most were caught after 1980 (Figure 2.3, Appendix A.1). Depth of records ranged from $2 \mathrm{~m}$ to $2730 \mathrm{~m}$ (Figure 2.4). Any record with an assigned depth that when plotted with depth contours appeared to be an error, was removed. Full summary information of these occurrence records can be found in Appendix A.2.

Using R Studio version 3.5.1, occurrence records were filtered and only those collected from bottom trawls and high opening bottom trawls were kept, so that 579,435 records remained. Records where other gear methods were used were excluded. Only records with excellent or satisfactory gear performance, as per the trawl database documentation by Mackay (2011), were kept so that 
545,077 remained. Records with NA or zeros as coordinates were also removed so that the final total number of records in the entire dataset was 543,909. This filtering was done for consistency of geographic comparisons and to retain only high-quality data. The dataset was then subset by species into separate files for each of the ten target species. The files were then reduced to only the coordinate information for each species, in order to produce the necessary input files for the MAXENT modelling software (Phillips et al., 2004). A summary of the number of occurrence records for each species, before further processing is provided in Table 2.1.

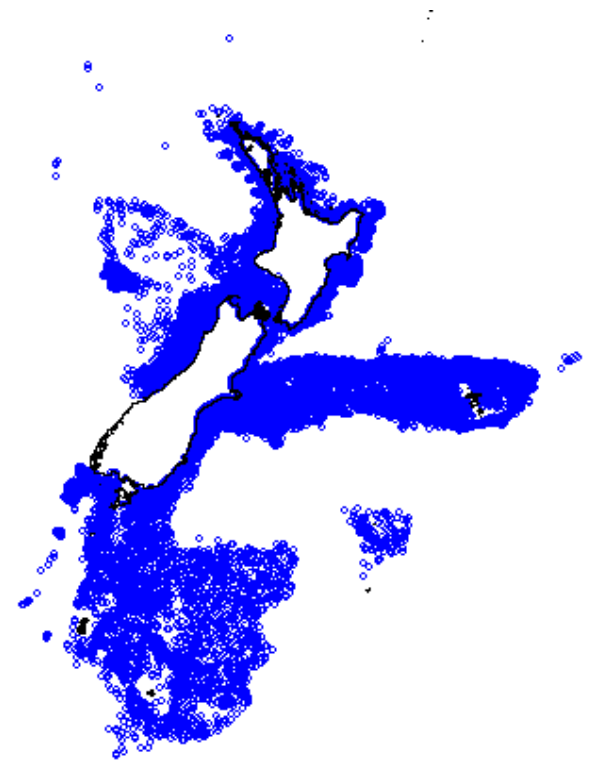

Figure 2.2: Total geographic coverage of occurrence of species records in the New Zealand region from the MPI trawl dataset (provided by NIWA, M. Dunn).

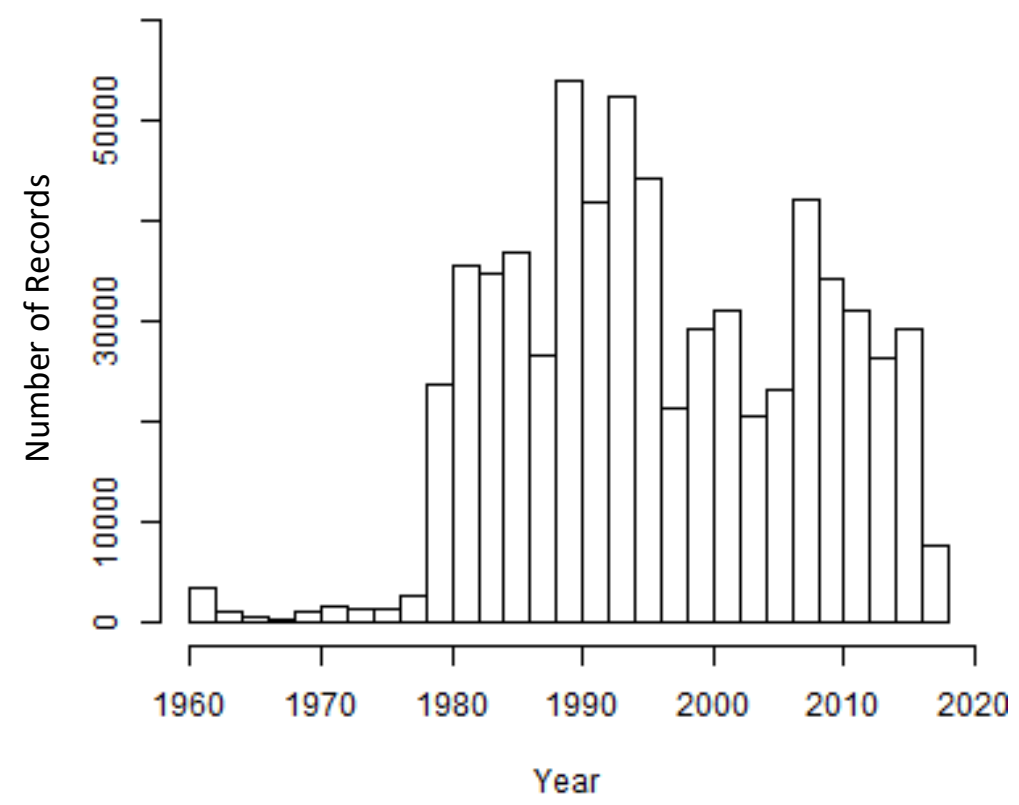

Figure 2.3: Total number of occurrence records ( $y$ axis) collected per year ( $x$ axis) from the New Zealand region from the MPI trawl dataset (provided by NIWA, M. Dunn). 

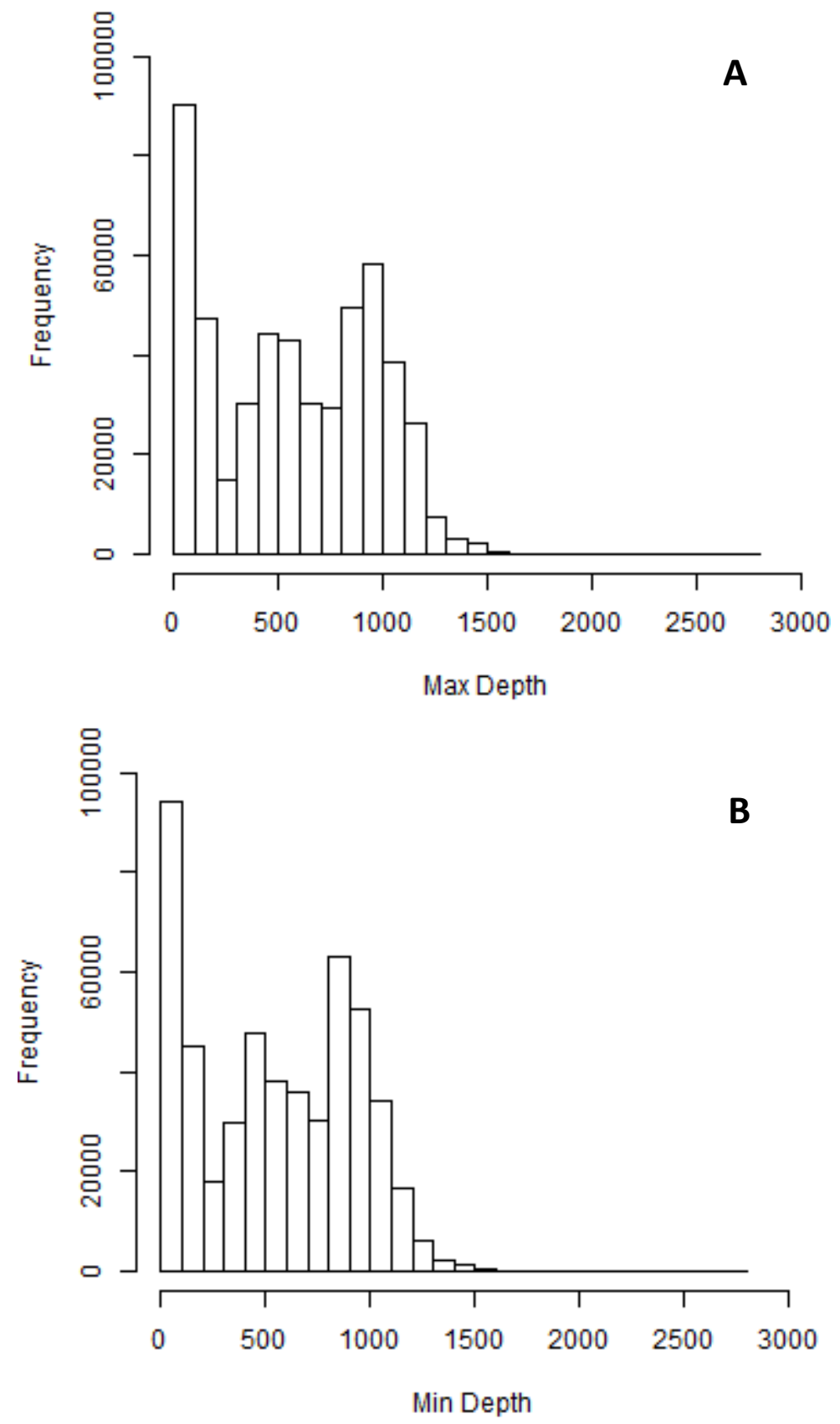

Figure 2.4: Maximum depth of records (A) and minimum depth of records (B) in New Zealand, with depth (x axis) and frequency of occurrence (y axis), of records in the total occurrence dataset provided by NIWA (M. Dunn).

\subsection{2: Spatial Bias in the Occurrence Data}

\section{Assumptions about occurrence data}

A key assumption in ENM is that occurrence data points are spatially independent from each other.

The presence of sampling bias violates this assumption (Boria et al., 2014; Carsten et al., 2007).

Unstructured sampling effort and recurrent sampling of more accessible areas are common in PO 
datasets and result in biased data (Fithian et al., 2015; Fourcade et al., 2014). In the marine environment, sample bias may arise due to higher levels of sampling inland, as it is more easily accessible, or because of management priorities. Locations distant from land may be less often sampled for practical reasons (Corkeron et al., 2011; Derville et al., 2018). Furthermore areas where important commercial species do not commonly occur are likely to be rarely sampled. Sampling bias promotes environmental bias by over-representing environmental conditions associated with regions (or time periods) that encompass clustered records (Aiello-Lammens at al., 2015b; Boria et al., 2014; R. J. Hijmans et al., 2000; Kadmon et al., 2004; Reddy \& Dávalos, 2003). In ENM this creates models over-fit to environmental conditions associated with clustered records and leads to erroneous estimates of both optimum conditions and variable response and influence (Boria et al., 2014). Temporal bias may also be introduced if surveys are consistently always done at similar times, for example only during certain seasons or in 'good' weather (Corkeron et al., 2011; Derville et al., 2018; Elith et al., 2011). On further inspection, these data appeared to be relatively consistent with year-round sampling (Appendix 2.3).

Similarly, it is assumed in ENM that the sampled area reflects the entire range of conditions the target species can tolerate (Anderson, 2015). If sampling bias results in omission of large areas of a species' range this assumption is unreasonable as these areas will be mistaken for regions that aren't inhabited due unfavourable conditions. This often produces overly conservative estimates of suitable habitat, due to a narrower range of environmental conditions being associated with occurrence records (also known as overfitting) (Radosavljevic \& Anderson, 2014). The following sections explain how sampling bias was addressed this study, so that these assumptions were more acceptable. However, assuming the full range of areas with tolerable conditions are occupied may also be unrealistic if dispersal barriers have prevented occupation of suitable areas. This study assumed few or no dispersal barriers currently operating in the marine environment (that are not included in the investigated variable set) substantial enough to have majorly restricted any of the target species' ability to inhabit its full range of tolerable environment. Therefore, once accounting for spatial bias had been attempted, it was assumed that the occurrence dataset represented spatially independent geographic localities associated with the full range of environmental conditions tolerable for each species.

It is further assumed that within the species range niche characteristics are similar, so that environmental conditions associated with occurrences are within the same tolerance range. If species are genetically isolated or have begun to isolate and adapt to different conditions this may be an issue. An ideal species for ENM is one with panmixia throughout its range (Radosavljevic \& 
Anderson, 2014). The ability of the occurrence data to accurately represent the environmental niche is compromised if records were mislabelled, species were misidentified, coordinates were entered incorrectly, or if occurrence localities were not from a source habitat. If occurrences were from a sink habitat, the associated conditions would not represent those necessary to maintain its population without emigration and may therefore exaggerate tolerance ranges (Phillips et al., 2006). Large datasets including repeated surveys, such as the one used here, reduce the influence of records with errors such as these, given there are few in the dataset.

\section{Addressing spatial bias: Method 1 - Adjusting Background Extent}

There are a variety of methods available to attempt to account for sampling bias and ensure key assumptions are met (Mateo et al., 2010; Steven J Phillips et al., 2009; Syfert et al., 2013; VanDerWal et al., 2009). One common way is to adjust the background (BG) samples that Maxent takes (Anderson \& Gonzalez, 2011; Anderson \& Raza, 2010; Barve et al., 2011; Elith et al., 2011; Fourcade et al., 2014; Mateo et al., 2010; Phillips et al., 2009). Due to utilising PO, rather than PA data, Maxent uses BG points during model training instead of absence points (Elith et al., 2011). Maxent compares the relationship between environmental variables at locations occupied by the species with independently and randomly selected BG locations, where species presence is unknown (Merow et al., 2013; Muscarella et al., 2014). Ability to differentiate between presence and background locations based on environmental conditions largely determines the model quality (Merow et al., 2013).

By default, Maxent randomly extracts BG points from the entire study region and every pixel has the same probability that a BG point will be selected from it (Elith et al., 2011; Merow et al., 2013). This assumes the whole area was available for sampling and that the species were equally likely to be found anywhere within the region (Elith et al., 2011; Merow et al., 2013). These assumptions do not hold true given sampling bias in the dataset. To mitigate this, the area where BG points are taken from can be adjusted (Merow et al., 2013; Phillips \& Dudík, 2008).

Adjusting the area where BG points are taken from allows the user to exclude areas where there were suitable conditions but species had not been recorded either due to sampling bias or dispersal barriers (Anderson, 2015; Anderson \& Raza, 2010; Barve et al., 2011). As well as the default entire background (EBG) method, two established approaches of adjusting BG areas were trialled: the Target Group Background (TGBG) method (Anderson, 2003; Phillips et al., 2006; Ponder et al., 2001) and the Restricted Background (RBG) method (Phillips, 2008). 


\section{$\underline{\text { Target Group Background }}$}

This method uses collective occurrence records for many similar species (the target group), obtained via the same method, from the same database, under the assumption that those surveys would have recorded the focus species had it occurred there (Anderson, 2015; Anderson et al., 2003; Dudík et al., 2006; Fitzpatrick et al., 2013; Phillips et al., 2009; Ponder et al., 2001; Syfert et al., 2013). Based on the occurrence records of the target group, the sampling distribution can be estimated. This permits BG data to be taken only from the area that was sampled, rather than the whole region. This reduces the potential for $B G$ points to be taken from areas with suitable conditions but no presence records, thus reducing the tendency to over fit to environmental conditions associated with sampled areas (Phillips \& Dudík, 2008). When using this method, the BG sample theoretically reflects and therefore counteracts bias in the presence data (Dudík et al., 2006; Phillips \& Dudík, 2008; Reddy \& Dávalos, 2003; Zaniewski et al., 2002). Others have found the TGBG method significantly improves model performance and reduced the effect of sampling bias (Elith \& Leathwick, 2007; Elith et al., 2011; Phillips \& Dudík, 2008). Here, the sampled area was estimated using the occurrence records of all marine species in the trawl database as the target group, filtered as previously mentioned for gear use and quality control. A number of coordinates associated with occurrence records in this target group were then randomly selected to be used as BG points in model training to apply the TGBG method (Figure 2.5B). A file was constructed from these coordinates for input into Maxent. Therefore, environmental conditions at the occurrence coordinates associated with the target species were contrasted with the environmental conditions at BG coordinates associated with the target group during model training when this BG method was implemented.

\section{Restricted Background}

This method is another way to adjust the area where BG points are taken from. This involved creating circular bounding buffer areas around each occurrence point, at a certain user-defined distance from each point, then merging these areas by dissolving interior lines so that an overall area encompassing all points remained (Phillips et al., 2009). BG points were randomly selected from within the merged region rather than from the entire study area. In this way, the area from which BG points could be taken was restricted. This method allows the BG data to better reflect the occurrence data bias, which encourages better distinction between presence and BG points (Phillips et al., 2009).

Variability in MaxEnt predictions produced with different background extents has been comprehensively documented (Anderson \& Gonzalez, 2011; Anderson \& Raza, 2010; Baasch et al., 2010; Barve et al., 2011; Elith et al., 2010; Elith et al., 2011; Giovanelli at al., 2010; Merow et al., 2013; Phillips et al., 2009; VanDerWal et al., 2009; Yates et al., 2010). A more limited BG extent 
encourages better differentiation between conditions in occupied and unoccupied areas, and therefore better recognition of distribution limiting conditions (Phillips, 2008). However, having more local BG points increases model complexity and therefore extrapolation when projecting the model to a different environment (Elith et al., 2011; VanDerWal et al., 2009). In this study three different restricted background extents were trailed to find an optimal distance: $10 \mathrm{~km}$ (RBG1), 50km (RBG2) and 100km (RBG3). The three distances here were compared with the TGBG and EBG methods (Figure 2.5). Although the recommended restricted distance is one that reflects the average dispersal capability of the focal species, these data are not always available (Zeng et al., 2016). Additionally, it was more feasible to trial the same set for each species, than to change it for each species being modelled in this study.

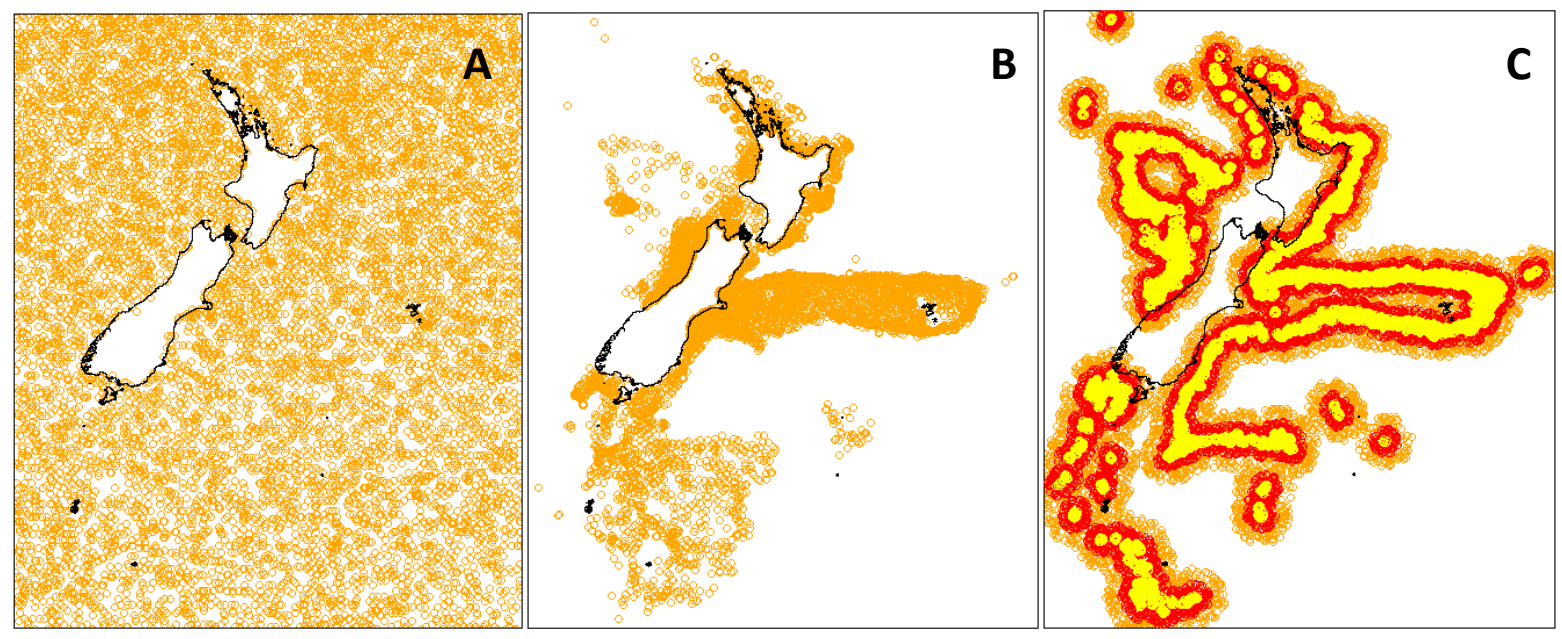

Figure 2.5: Plots of the New Zealand region with examples of the different BG adjustment methods; The circles indicate points that were randomly selected as BG points dependant on the method used. A: EBG (Default BG of entire study region), B: TGBG, C: Three different RBG extents around orange roughy occurrence points as an example; Yellow = RBG1 $(10 \mathrm{~km})$, Red = RBG2 $(50 \mathrm{~km})$, Orange $=\operatorname{RBG3}(100 \mathrm{~km})$.

\section{Addressing spatial bias: Method 2 - Spatial rarefication}

Spatial rarefication is another method commonly used to reduce sampling bias in presence only datasets (Boria et al., 2014; Fourcade et al., 2014). This involved adjusting the occurrence data itself before using it in the model. Spatial rarefication was chosen rather than spatial thinning due to computational constraints of thinning on large datasets. The R package spThin was used to conduct spatial rarefication, using the function spRarefy (Aiello-Lammens et al., 2015a; Aiello-Lammens et al., 2015b). Spatial rarefication was implemented for each species dataset, reducing occurrence points to only ones a certain, user defined distance away from each other (Boria et al., 2014). This was done by selecting a single record from each grid cell randomly in replicates of 10 . Increasing the number of replicates did not change the points selected or number of records that remained. 
Spatial rarefication was conducted due to the large variation in the density of records between sampled areas (see Figure 2.2). If this was not corrected the model fit would favour the conditions where density of records was high and down weight conditions where there were fewer occurrence points. The aim of rarefication was to reduce effects of overrepresented localities in the dataset without reducing the signal of areas with suitable habitat (Aiello-Lammens et al., 2015(b); Anderson, 2015). Rarefication should result in more equal weight given to all areas and reduce the effect of clustered records, which place artificial importance on the associated environment. Too much rarefication however may lead to smoothing the distribution of tolerable conditions and therefore overestimation of probability of presence (POP) at the edges of distributions, as well as underestimation of POP where conditions are most preferred (Fourcade et al., 2014).

The distance at which points are set away from each by rarefication other should depend on the precision at which the data was collected and what is computationally feasible (Jeffery Hanson, University of Queensland, email correspondence). In this case, a distance of $10 \mathrm{~km}$ was selected because as the distance between the surveys tows that collected the data was often 3 nautical miles (5.556 km), following the standard operating procedure (Hurst et al., 1992; Stevens, 2014). If each tow was $\sim 5 \mathrm{~km}$, the midpoint has to be at least $\sim 5 \mathrm{~km}$ away in order for there to be no overlap between tows ( 1 tow per 6 n.mile), so the minimum distance between each tow would have been roughly $10 \mathrm{~km}$ (Aiello-Lammens et al., 2015b). Therefore, this distance was selected as the maximum distance at which points should be set apart from each other. Finer spatial rarefication at $5 \mathrm{~km}$ and $1 \mathrm{~km}$, was also trailed (Table 2.1).

Although rarefication deals with geographically clustered records it cannot account for the omission of areas of suitable habitat. Therefore, simultaneously using rarefication and different background adjustment methods (such as previously discussed TGBG or restricted BG methods) is potentially an effective method to reduce the effect of sample bias on models. Therefore combinations of these methods were trailed for each species. See Table 2.2 for all combinations of methods used. Effective reduction in sampling bias should result in a model with less overfitting and better predictive performance on independent evaluation data (Boria et al., 2014). 
Table 2.1: Number of records for each target species before and after rarefication:

\begin{tabular}{ccccc}
\hline Species & No rarefication & $\begin{array}{c}1 \mathrm{~km} \\
\text { rarefication }\end{array}$ & $\begin{array}{c}5 \mathrm{~km} \\
\text { rarefication }\end{array}$ & 10km rarefication \\
\hline Hoki & 14250 & 13137 & 9483 & 6535 \\
Snapper & 3828 & 3099 & 1905 & 1171 \\
Tarakihi & 5249 & 5001 & 3732 & 2404 \\
Trevally & 1623 & 1368 & 964 & 683 \\
Scampi & 1535 & 1483 & 1286 & 1023 \\
Orange roughy & 9605 & 7554 & 3652 & 2093 \\
Southern Blue Whiting & 1675 & 1654 & 1562 & 1427 \\
John Dory & 3951 & 3360 & 2247 & 1402 \\
Ling & 10967 & 10520 & 8504 & 6106 \\
Arrow Squid & 4522 & 4338 & 3702 & 2854 \\
\hline
\end{tabular}

Table 2.2: Trial codes for Maxent runs showing the combinations of background methods, including Entire Background (EBG), three different Restricted Backgrounds (RBG) and Target Group Background (TGBG), and species occurrence data variations, including all records (no adjustment to occurrence records), removal of duplicates, and three spatial rarefication variations, used in Maxent trials. These are referred to throughout the results section in chapter three.

\begin{tabular}{llllll}
\hline BG method & All records & $\begin{array}{l}\text { Duplicates } \\
\text { not included }\end{array}$ & $\begin{array}{l}\text { Spatial } \\
\text { rarefication } \\
(10 \mathrm{~km})\end{array}$ & $\begin{array}{l}\text { Spatial } \\
\text { rarefication } \\
(5 \mathrm{~km})\end{array}$ & $\begin{array}{l}\text { Spatial } \\
\text { rarefication } \\
(1 \mathrm{~km})\end{array}$ \\
\hline EBG & $1 \mathrm{a}$ & $2 \mathrm{a}$ & $3 \mathrm{a}$ & $4 \mathrm{a}$ & $5 \mathrm{a}$ \\
RBG 1 $(10 \mathrm{~km})$ & $1 \mathrm{~b}$ & $2 \mathrm{~b}$ & $3 \mathrm{~b}$ & $4 \mathrm{~b}$ & $5 \mathrm{~b}$ \\
RBG 2 $(50 \mathrm{~km})$ & $1 \mathrm{c}$ & $2 \mathrm{c}$ & $3 \mathrm{c}$ & $4 \mathrm{c}$ & $5 \mathrm{c}$ \\
RBG 3 $(100 \mathrm{~km})$ & $1 \mathrm{~d}$ & $2 \mathrm{~d}$ & $3 \mathrm{~d}$ & $4 \mathrm{~d}$ & $5 \mathrm{~d}$ \\
TGBG & $1 \mathrm{e}$ & $2 \mathrm{e}$ & $3 \mathrm{e}$ & $4 \mathrm{e}$ & $5 \mathrm{e}$ \\
\hline
\end{tabular}

\section{3: Climate Data}

\section{Obtaining and Preparing Climate layers}

Marine data for the modern and predicted future climates were obtained from Bio-ORACLE

(http://www.bio-oracle.org/downloads-to-email.php) (Assis et al., 2018; Tyberghein et al., 2012).

These data were available at a resolution of $5 \mathrm{arc}-\mathrm{min}$. It has been suggested that higher resolution climate layers with finer grain sizes commonly give more accurate models, (Connor et al., 2018; Manzoor et al., 2018; Scales et al., 2017; Song et al., 2013) however this was all that was readily available and easily accessible. Data for 2040-2050 and 2090-2100 were downloaded for each different Representative Concentration Pathway Scenario (RCPS). These four climate scenarios are 
RCPS 2.6, a decline in greenhouse gas concentrations, RCPS 4.5 and RCPS 6.0 stabilisation of greenhouse gas concentrations, and the RCPS 8.5, an increasing emissions scenario (Van Vuuren et al., 2011). Bio-oracle provides both surface and deep sea environmental layers, however only surface layers were selected for consistency across models, and because deap sea environmental data has poorer collection coverage. Sea Surface Temperature (ST) and Surface Salinity (SS) covariates were downloaded (12 variables in total). Modern data were also taken from Bio-ORACLE of the same nature as the future data ( 5 arc-min, ST \& SS covariates). Modern bathymetry climate layers were downloaded from MARSPEC (http://marspec.weebly.com/modern-data.html)(Sbrocco, 2014), and added to all climate sets as depth data were not available on Bio-ORACLE.

It is important to consider which environmental factors are used to train ENMs. The model assumes they are ecologically relevant to, independent of and have temporal correspondence to, species presences (Anderson, 2015; Phillips et al., 2006; Werkowska et al., 2017). Temperature and salinity were chosen as variables to use in this study as both have frequently been linked to marine species distributions (Balzano et al., 2010; Golikov et al., 2013; Khan et al., 2013; Kimmerer, 2002; Kültz, 2015; Lehtonen et al., 2016; Smyth \& Elliott, 2016). Including more variables often increases complexity and propensity for overfitting and complicates interpretation, while adding little further information to predictions (Werkowska et al., 2017). Furthermore, transferability of models when projecting to different time periods decreases with additional variables (Werkowska et al., 2017). For this reason, and for time and computational feasibility reasons, other variables from the large selection available from Bio-ORACLE were not included. Furthermore, predictor variables with varied effect on species distributions across the modelled region such as temperature average of the warmest month were omitted, as recommended by Peterson (2006) and Phillips et al., (2006).

Temporal correspondence between the occurrence dataset and the climate data must also be considered. Temperature and salinity datasets were assembled from climate data collected between 2002-2009 (Tyberghein et al., 2012). Although occurrence data was drawn from a larger time scale (1960-2017), it was assumed that two still relatively correspond, as most records have been collected between 1980 and 2017. Because of this and for the purpose of retaining as many records as possible, occurrence data were not further filtered to produce a narrower collection date range. However, it is acknowledged that it is possible that there have been changes in distributions or environmental responses over this time period. A species could be absent or depleted from an area in some years where it recently resided due to depletion of fish stocks from fishing, or other distributional fluctuations over this time period.

Multicolinearilty between variables is often raised as an issue in ENM (Werkowska et al., 2017). Although collinearity does not considerably compromise model quality when using Maxent (De 
Marco \& Nobrega, 2018), it does complicate interpretation of response curves and variable contributions (Werkowska et al., 2017). Correlations between variables were assessed using the ENMTools R package (Warren et al., 2010). Almost all ST and SS variables were highly correlated with each other (>0.85), except for SS range and ST Range (Appendix B.1 \& B.2). Because the aim was to investigate long-term change, mean SS and ST covariates were kept, and all other variables correlated with them discarded. SS range and ST range were also kept as these were not highly correlated with other variables and some species are influenced more by temperature extremes than averages as drivers of range shifts (Grieve et al., 2016; Hare et al., 2010; Hare et al., 2012; Morley et al., 2018a). Thus, a reduced variable set was assembled that included bathymetry, SS Mean, SS Range, ST Mean, and ST Range. Although this set of environmental variables may not be sufficient to describe all parameters of the fundamental niche for the study species, they will allow approximate estimation of geographic distributions based on a few key variables (Phillips et al., 2006).

All climate layers were subject to cropping and transformation in R Studio version 3.5.1. All downloaded climate data was in -180 to 180 format longitudinal format. In order to preserve all of New Zealand marine space in the models and avoid cut-off at the 180 International Date Line, these had to be converted to 0 to 360 longitudinal formats. These were then cropped to include only the desired study region and the final raster layers were able to be used as inputs for the Maxent algorithm. Summary plots for the climate layers used in this study can be found in Appendix C.

\section{4: Model Assembly}

\subsection{1: Data Partitioning}

A 'masked geographically structured' data partitioning method was used where both occurrence and BG points were partitioned by geographic space. Occurrence and BG points were partitioned into four bins based on their position relative to latitude and longitude lines that divided occurrence localities as equally as possible (Muscarella et al., 2014; Radosavljevic \& Anderson, 2014). After partitioning, four models were built iteratively for each combination of settings, using three bins for model training and the withheld bin for testing. Therefore, the calibration localities could not be next to or in the same geographic cluster as, evaluation localities and thus were independent from each other as recommended by Radosavljevic \& Anderson (2014). This addresses the spatial autocorrelation issue that often arises when using random cross validation. In random cross validation evaluation data may be taken from areas of clustered localities (ie. due to sampling bias), and thus its independence from the calibration data is compromised. This often leads to overinflation of performance values and doesn't address spatial bias (Boria et al., 2014; Roberts et al., 2017). The background localities in the same geographic area as the bin holding testing localities 
were not included in the training phase (Phillips, 2008; Phillips \& Dudík, 2008; Radosavljevic \& Anderson, 2014). This method is called 'spatial blocking' and is often used to reduce spatialautocorrelation between testing and training points (Boria et al., 2014; Hijmans \& Elith, 2013; Wenger \& Olden, 2012; Muscarella et al., 2014; Radosavljevic \& Anderson, 2014; Veloz, 2009). Spatial blocking is recommended when model transfer across time or space is required, as it offers more accurate extrapolation to conditions outside of those not used in model training (Wenger \& Olden, 2012; Muscarella et al., 2014; Zeng et al., 2016). The block method was implemented using the R package ENMeval (described below) using the ENMevaluate function (Muscarella et al., 2014).

\subsection{2: Maxent Runs}

The MaxEnt software (version 3.4.1) downloaded from

https://biodiversityinformatics.amnh.org/open source/maxent/ was used to perform ENM. It is necessary to perform species specific tuning when using Maxent by trialling a variety of different combinations of settings for each species (Morales et al., 2017; Phillips \& Dudík, 2008). The two main parameters in Maxent that influence model results are regularization and choice of feature class (fc). Regularisation influences model complexity and affects how closely fit the model is. An increase in the regularisation multiplier (RM) penalises complexity and constrains potential for overfitting (Merow et al., 2013; Phillips et al., 2006; Phillips \& Dudík, 2008; Phillips et al., 2004; Warren \& Seifert, 2011). Feature class refers to the type of mathematical transformation of variables for modelling of complex relationships (Elith et al., 2010). For further detail on feature classes see Elith et al. (2011).

To run MaxEnt trials the R package ENMeval was used. ENMeval implements Maxent via the dismo package and allows users to test a variety of combinations of RMs and fcs and simplifies the process of comparing many models at a time (Hijmans et al., 2011; Muscarella et al., 2014). Each ENMeval trail was run in parallel with RM values of 0.5-8 in increments of 0.5 , and a variety of combinations of fcs including Linear (L), Quadratic (Q), Hinge (H), Product $(P)$ and Threshold $(T)$ feature classes. These were 'L','LQ','H','LQH','LQP', 'LQPT','LQPH', and 'LQHPT'.

For each trial, 10,000 back ground points were randomly selected from the specified background extent as this typically achieves optimal performance (Elith et al., 2006). It is suggested that more BG points should be taken for larger datasets as more BG points typically equates to improved model predictive performance (Elith et al., 2006). Because all species datasets used here had under 10,000 records or just over increasing the number of BG points from 10,000 would mostly be redundant (Dudik et al., 2007). 
ENMeval runs were performed using the ENMevaluate function on the five different BG treatments (EBG, TGBG, \& three different RBG extents) with five different occurrence datasets (all localities, no duplicates, and three spatially rarefied by different distances). Table 2.2 shows a summary of these method combinations used. Each run was performed using the modern climate layers obtained from Bio-ORACLE. Sixteen RMs and eight fc combinations on these 25 different treatments resulted in a total of 3200 models run per species, 32,000 overall.

\section{5: Model Selection}

\section{Evaluation Metrics}

After each ENMevaluate call, the function produced a variety of evaluation metrics for each combination of settings. One of the most important of these is the Akaike Information Criterion (AICC). The AICc is a model selection uncertainty metric and reflects both goodness of fit and complexity. AICC is the version of AIC that was developed to correct for small sample size, and reduce the chances of the model being over fitted (Brewer et al., 2016). It is used to find optimal level of complexity and gives an overall indication of model quality (Radosavljevic \& Anderson, 2014; Warren \& Seifert, 2011). The philosophy underlying the AICc metric is that models that fit that data are rewarded while unnecessary parameters are penalised (Warren \& Seifert, 2011). Complexity is estimated based on the lambda file produced at the end of each model run, in which all the parameters with a nonzero weight are counted to give the number of parameters used (Warren \& Seifert, 2011). AICc is calculated on the full set of localities (both train and test) so is not affected by partitioning method (Muscarella et al., 2014; Warren \& Seifert, 2011). Models with a delta( $\Delta$ ) AICC value under two have substantial support and a $\triangle \mathrm{AICC}$ of 0 is indicative of the 'best' models (Burnham \& Anderson, 2004).

Another important evaluation metric produced is the 'mean AUC', which refers to the Area Under the operator Curve. This is based on the testing data (withheld from model construction), averaged across all partitioned bins ( $A \cup C_{\text {test }}$ ) (Warren \& Seifert, 2011). $A \cup C_{\text {test }}$ is calculated in each iteration on the full set of background localities (Radosavljevic \& Anderson, 2014). This presents the model's ability to distinguish between conditions at occurrence and background localities, as it gives an estimate of the probability that a randomly chosen presence locality would be ranked above a randomly chosen absence locality (Boria et al., 2014; Muscarella et al., 2014; Phillips \& Dudík, 2008; Phillips et al., 2004; Radosavljevic \& Anderson, 2014). An AUC score of 0.5 or under indicates that the probability a randomly chosen presence is ranked above a randomly chosen background point is no better than random (Phillips \& Dudík, 2008). The closer to 1 the AUC value is, the better the discriminatory ability. Usually, values above 0.75 are considered acceptable (Phillips \& Dudík, 2008). 
The AUC value for the full model, based on data used in model training, is also given by ENMevaluate $\left(A \cup C_{\text {train }}\right)$ (Warren \& Seifert, 2011).

AUC does not allow comparisons between models with different study regions, backgrounds, species, or test data, only between similar models having different settings (such as RMs and fcs) (Merow et al., 2013; Radosavljevic \& Anderson, 2014). AUC is a threshold independent measure of discriminatory ability but gives no indication of model fit (Lobo et al., 2008; Peterson et al., 2011; Phillips et al., 2006; Phillips et al., 2004). AUC therefore tends to favour more complex models (Fourcade et al., 2014; Radosavljevic \& Anderson, 2014; Warren \& Seifert, 2011). Therefore, it is important to select models with low overfitting before assessing AUC values.

ENMeval produces three metrics for estimating overfitting. The first is given as the 'mean $A \cup C_{D I F F}$ ' and is the calculated difference between calibration ( $A \cup C_{\text {train }}$ ) and evaluation ( $\left.A \cup C_{\text {test }}\right) A \cup C$ scores, averaged across all bins (Boria et al., 2014; Warren \& Seifert, 2011). Large difference between calibration and evaluation AUC equates to models over fit to the training data (Boria et al., 2014). This is because over fit models frequently perform well on calibration data, but not on evaluation data. If the difference is minimised, so is the potential for overfitting (Warren \& Seifert, 2011).

The other two metrics produced are threshold-dependent omission rates. Omission rates are indicative of the proportion of test localities that are omitted from the model, based on the threshold used (Boria et al., 2014). If more localities have been omitted than expected, the model is over fit (Boria et al., 2014; Radosavljevic \& Anderson, 2014; Shcheglovitova \& Anderson, 2013). Therefore, lower omission indicates better discrimination between suitable and unsuitable areas and generally higher model performance (Boria et al., 2014). Two kinds of omission rates are presented by ENMeval. The first is the minimum training presence threshold $\left(\mathrm{OR}_{\mathrm{MTP}}\right)$, presented by ENMeval as 'mean ORmin', which sets the threshold at the lowest value of prediction for any pixel with a calibration locality (the training locality with the lowest predicted value) and so calculates omission rate on all the data. Thus there is an expected omission rate of $0 \%$ (Boria et al., 2014). An ORMTP value over zero indicates a level of overfitting. The second is the $10 \%$ calibration omission rate (mean $\mathrm{OR}_{10}$ ), which sets a threshold at a value that excludes $10 \%$ of training localities with the lowest predictions, giving an expected omission rate of 0.10 (Boria et al., 2014). Therefore, a mean $\mathrm{OR}_{10}$ above 0.10 , indicates some level of over fitting (Pearson et al. 2007).

\section{Base model selection}

The methods generated hundreds of alternative models for each species. The best model for each species was chosen based on two main points of consideration. The fist was based on the evaluation metrics and the other was based on biological interpretation of the models. Firstly, models were 
filtered so that only those with a $\triangle \mathrm{AICC}$ of 0 were considered. Of these, models were selected based on those with the lowest overfitting metrics $\left(O R_{10}, O R_{M T P}\right.$, and $\left.A \cup C_{D I F F}\right)$. OR $R_{M T P}$ was frequently so low in all models filters thus far that had little relevance during model selection. Then, only those with the highest discriminatory ability ( $A \cup C_{\text {test }}$ ) were kept. In order to follow a species-specific selection process, and because evaluation metrics varied between species, the criteria for best models changed on a case-by-case basis to be more or less strict. Thus, the selection criteria was slightly different for each species, as is detailed further in the results. $\triangle A I C C$ scores and AUC scores had selection criteria that was always the same, but the criteria for selecting those with low overfitting metrics varied considerably depending on the species. Six models were identified as the relative 'best' models for each species. These were subject to further analysis in order to select a base model to use for the climate change investigations.

A biological interpretation approach was subsequently adopted to investigate which models had best captured the niche of each species. The six models with the 'best' evaluation metrics were projected onto modern climate layers to visually inspect which best reflected the known current distribution of the target species. This was checked by expert opinion where possible, but alternatively density maps created with occurrence records were used as comparisons. Using current distributions to check model predictions assumes entire distributions are known and was therefore only used as an approximate guideline. Although over prediction of distributions was difficult to dispute, under prediction was generally an obvious error and could often be interpreted as a sign of overfitting. Analysis of the response curves further informed choice of base model. Models that showed no response to climate variables were excluded, provided the next met model was not substantially more over fit to the training data.

This process reflects recommendations that low overfitting should be a primary criterion before discriminatory ability is taken into account, and that ecological interpretation is an important factor to consider when choosing models (Derville et al., 2018; Radosavljevic \& Anderson, 2014;

Shcheglovitova \& Anderson, 2013). This allowed selection of base models that were not overly complex, had sufficient ability to distinguish preferred areas from not preferred areas, and will have better transferability to other time periods (Warren \& Seifert, 2011, Werkowska et al., 2017).

\section{Variable Influence}

Variable influence and contribution to models was investigated by analysing response curves, permutation importance and limiting factor plots. Response curves indicated how the model responded to the co-variates and permutation importance indicated how much the variables contributed to the models. Permutation importance was used rather than percent contribution 
because permutation importance is only measured from the final model rather than the path taken to obtain it (Phillips, 2006). Permutation importance is calculated by measuring how much AUC drops when the model is re-evaluated on each variable in turn, with its values randomly permuted throughout the training region to remove the effect of any environmental gradient or pattern they exhibit. The more the AUC score dropped, the more the variable contributed to the model.

Limiting factor plots were constructed independently of the models using the R package Rmaxent (Baumgartner, 2018). These plots indicate the most limiting factor at each grid cell. This was calculated by giving each variable a new value at each cell equal to that of the mean value of that variable across the entire training region. If the predicted suitability increased as a result of this value change, more so than when any other variable is subject to the same treatment, then that variable was mostly responsible for limiting suitability in that grid cell and was thus the most limiting factor (Elith et al., 2010). Limiting factor plots were created for modern climates and the future climate under RCPS 8.5, to contrast the two most different climate effects on distributions. This was done for each species.

\section{6: Model Projection}

After model training on modern climate layers to estimate the climatic niche, the selected base models for each species were used for projections onto the four sets of future climate layers, in 2050 and 2100. These projections presented Probability Of Presence maps (POP) for each species. It is implied that areas with high POP are areas with the most suitable habitat, or areas where that species will most likely be distributed based on the assumptions about the model. Thus for ease of interpretation, the phrases 'potential distributions' and 'areas of suitable habitat' are used interchangeably to describe areas with high POP in this thesis.

Successful estimation of the modern species distribution by the model does not guarantee successful projection to different climates as the model may have poor transferability (Elith et al., 2010). It is therefore important to critically evaluate ENM projections to different time periods or geographic regions. Projecting models to different time periods with different climates relies on good transferability and several assumptions. It is assumed that the relationship between the species and the variables used to train the models does not change between time periods (Anderson, 2015). This is also referred to as assuming niche conservatism or no niche evolution. The quicker environments change, the potential for sufficient adaptation is reduced, so the validity of this assumption may vary with climate scenario (Hoffmann \& Sgro, 2011). When interpreting the predicted distributions, it is further assumed that other factors influencing species distributions including biotic interactions do not change between time periods. This is a harder assumption to 
endorse due to the complexity of these interactions. Additionally, it is assumed that the constructed future climate datasets used to project models are as accurate as possible.

\subsection{1: Projection Evaluation}

\section{Extrapolation}

Because model assembly used recent climatic conditions to train the model when it is projected to a different time period, and thus onto different variables, it is possible that these variables have values outside of the range on which the model was trained. Despite this, projection occurs as though the base model is being projected onto the same climate layers used for training. Therefore, MaxEnt may predict outside of the range of values it encountered during training. This process is called extrapolation (Phillips et al., 2006). How well it does this depends on how well the occurrence data represented the environmental conditions within the species preferred habitat. Spatially and temporally extensive occurrence records are advantageous for this purpose.

By default, MaxEnt uses clamping to limit extrapolation by capping predictions to environmental conditions with values outside of the range of those used in training. Clamping is done so no values higher than the highest value and lower than the lowest value used in training remain in the climate layers that the model is being projected onto (Elith et al., 2011; Phillips et al., 2006). This clamps the predicted responses to the most alike conditions in the modern climate calibration data (Anderson \& Raza, 2010; Phillips et al., 2006).

Although this is the recommended standard practice, clamping itself can be problematic (Phillips et al., 2006). For example, clamping fixes response to temperatures higher than those encountered in training so that response is equal to response to the highest training temperature, and vice versa for lower temperatures. Therefore, temperatures far out of the range of those used in model training could are assigned the same predicted response as the most extreme temperatures used during model training. This may not be a realistic representation of species response to these new conditions and is referred to as the 'problem of novel climate conditions.' Presence of clamping could be identified from the covariate response curves.

\section{$\underline{\text { Uncertainty }}$}

To assess where there were novel conditions, and how they influenced future climates, Multivariate Environmental Similarity Surface (MESS) maps were produced (Elith et al., 2010; Radosavljevic \& Anderson, 2014). MESS maps measure the similarity between the future environment and the training sample (Elith et al., 2010) and present this as estimates of relative uncertainty throughout the study region by assigning negative or positive values. Thus the similarity in climatic variables between any given locality in the projection dataset and the localities in the training dataset was 
assessed (Elith et al., 2010). Negative values indicate sites where at least one variable had a value outside of the range encountered in the training data, thereby indicating novel conditions (conditions not experienced during model training). Conversely, the more positive the value the more similar the conditions were to those encountered during training. For each species, MESS maps for each climate scenario were produced. MESS analysis, in conjunction with response curves, were used to guide interpretations of the future climate predictions and identify how and where novel conditions affected predictions (Carneiro et al., 2016; Elith et al., 2010).

\section{Change in POP calculations}

Each projection map presented a continuous scale of probability of presence (POP) from 0-1. In order to estimate differences between different climate scenarios, this scale was split and assigned evenly into nine different 'bins' in increments of 0.1 (excluding all sites with a POP of <0.1) so that the proportion of area on each map that fell into each bin could be calculated. The proportion of area within one bin was calculated as a proportion of the whole study region. The proportion of sites within each bin was calculated for all modern and future projections. A percentage change for all future projections was then calculated as the change in proportion of area that fell into each POP bin from the modern projection for that species. This was done to show the amount of habitat of different qualities or POP rankings that was gained or lost in response to different climate scenarios. All calculations were performed in R Studio version 3.5.1. 


\section{Chapter 3: Results}

This chapter presents results of the ten focal species, organised by species. It starts with the inshore species snapper, john dory, trevally and tarakihi then continues to the deep-water species scampi, orange roughy, southern blue whiting, hoki, ling and arrow squid. This section duplicates analyses for each species. An overall summary and a cross-species comparison of results is described in the final section (3.11: Results Summary). Full results of all ENMeval runs that produced thousands of different models for each species can be found in a dropbox folder accessed by following this link: https://www.dropbox.com/sh/ebhbskzka9ebm0t/AADxJWY6BrGK2bAcuaANFI4ha?dl=0

\section{1: SNAPPER (Chrysophrys auratus)}

\section{$\underline{\text { Model Selection }}$}

Of all models generated, $\triangle \mathrm{AIC}$ values varied form 0 - 3145.1. Only models with a $\triangle \mathrm{AIC}$ of 0 were selected. Train AUC scores varied from $0.6-0.98$ and test AUC from $0.61-0.98$. To select models with good discriminatory ability, only those $>0.75$ were selected. To select models with low overfitting, average test $\mathrm{OR}_{10}$ values varied from $0.06-0.23$ but only those that were $<0.15$ were selected and average $A \cup C_{\text {DIFF }}$ varied from $0.001-0.113$ but only those $<0.05$ were selected. Only the models that met these criteria were considered for base model selection (Table 3.1.1). When projected as modern probability distributions, all predictions appeared relatively similar (Figure 3.1.1). When compared to the contemporary known distribution (Figure 3.1.2A), models $3 \mathrm{c} 10$ and 3d10 (Figure 3.1.0A\&B) over predicted snapper distribution around the top of the $\mathrm{Sl}$, while models $5 \mathrm{~d} 11$ and $5 \mathrm{~d} 12$ (Figure 3.1.1E\&F) appeared to under predict around the lower NI. Of the remaining two, model 3 e70 (Figure 3.1.1C) showed lower overfitting values than all other models $\left(\mathrm{OR}_{10}=0.072\right.$, $\left.A U C_{\text {DIFF }}=0.010\right)$ and was the only model that didn't exceed the $10 \% \mathrm{OR}_{10}$ threshold recommended by Muscarella et al. (2014). Model $3 e 70$ also had relatively good discriminatory ability with high AUC train and test scores above 0.9. Independent expert advice suggested model 3e70 was slightly more representative of the contemporary distribution (M Dunn, NIWI, pers. Comm). Model 3e70 was therefore selected from the six models as the base model for snapper projections. 

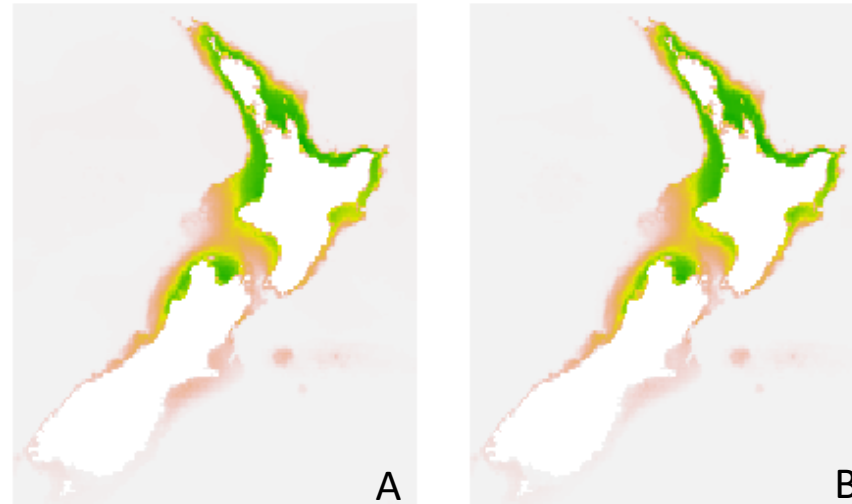

B

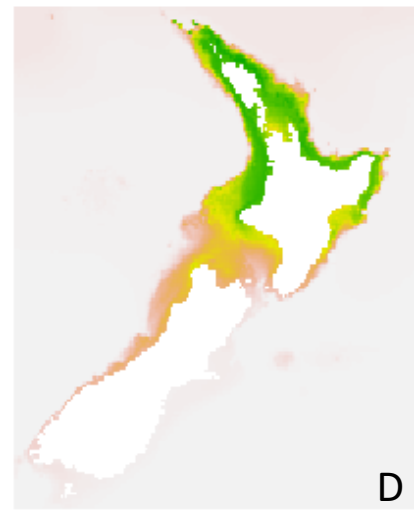

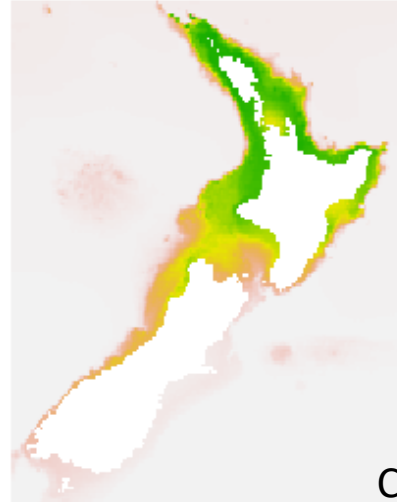

C

Figure 3.1.1: Snapper; Six best Maxent models projected on modern climate layers; 3c10 (A), 3d10 (B), 3e70 (C), $4 \mathrm{e} 28(\mathrm{D}), 5 \mathrm{~d} 11$ (E), $5 \mathrm{~d} 12$ (F). Colours reflect POP estimates between 0-1. Darker green indicates higher POP and red the reverse. 
All 2050 RCPS projections were similar (Figure 3.1.2B-E). In the modern projection snapper were essentially absent from the South Island (SI), expect for a small area on the N/W corner. All 2050 projections showed a moderate extension just over halfway down the West Coast of the SI, where POP increased from $<0.4$ to $>0.7$ in all RCPS. Around the entire North Island (NI) the POP was also predicted to increase substantially. In all future climate scenarios, areas with highly preferable climatic conditions, which therefore had the highest POP values, increased the most ( $P O P>0.9$ by $300.04-412.88 \%$ ), while areas with lower POP between 0.6 - 0.9 values increased comparatively less. Furthermore, the proportion of areas with POP values $<0.6$ decreased in all RCPS (Table 3.1.2).

The 2050 MESS maps for all RCPS displayed little relative uncertainty (Appendix D.1). Relative certainty was highest in the RCPS 2.6 prediction particularly around the Chatham rise, Cook Strait and West Coast of the SI (Figure 3.1.3A). Most relative uncertainty was around the north of the $\mathrm{NI}$ in RCPS 8.5.

\section{$\underline{2100 \text { Model Projections }}$}

The 2100 predictions showed more variation than the 2050 predictions, although similar patterns were observed (Figure 3.1.2G-J). A similar extension down the SI West Coast and general increase in POP around the NI was present in all 2100 RCPS, but these observations were more apparent in scenarios that deviated further from modern conditions. In the most 'extreme' scenario RCPS 8.5, the probability distribution extended to the lower SI and to Stewart Island. Unlike the other scenarios, the RCPS 8.5 showed POP of snapper exceeding 0.5 around the bottom of the SI. POP along the east coast of the SI was still very low, even in the more extreme scenarios. Other notable places of predicted POP increase was the Hauraki Golf, Hawke Bay and Cook Strait/South Taranaki bight areas. Similarly to the 2050 predictions, in all scenarios areas of high POP increased the most (POP >0.9 by $220.46-897.95 \%$ ), while areas with lower POP between $0.6-0.9$ increased comparatively less. Most striking was the $897.95 \%$ increase in the proportion of areas with a POP of $>0.9$ in RCPS 8.5 (Table 3.1.2). The proportion of areas with POP values $<0.5$ decreased in all RCPS (Table 3.1.1).

The 2100 MESS maps displayed more relative uncertainty than the 2050 MESS maps (Appendix D.1). Most relative uncertainty occurred around northern New Zealand. Uncertainty was most prevalent in the RCPS 8.5 prediction (Figure 3.1.3B), particularly from Hawke Bay northward. 


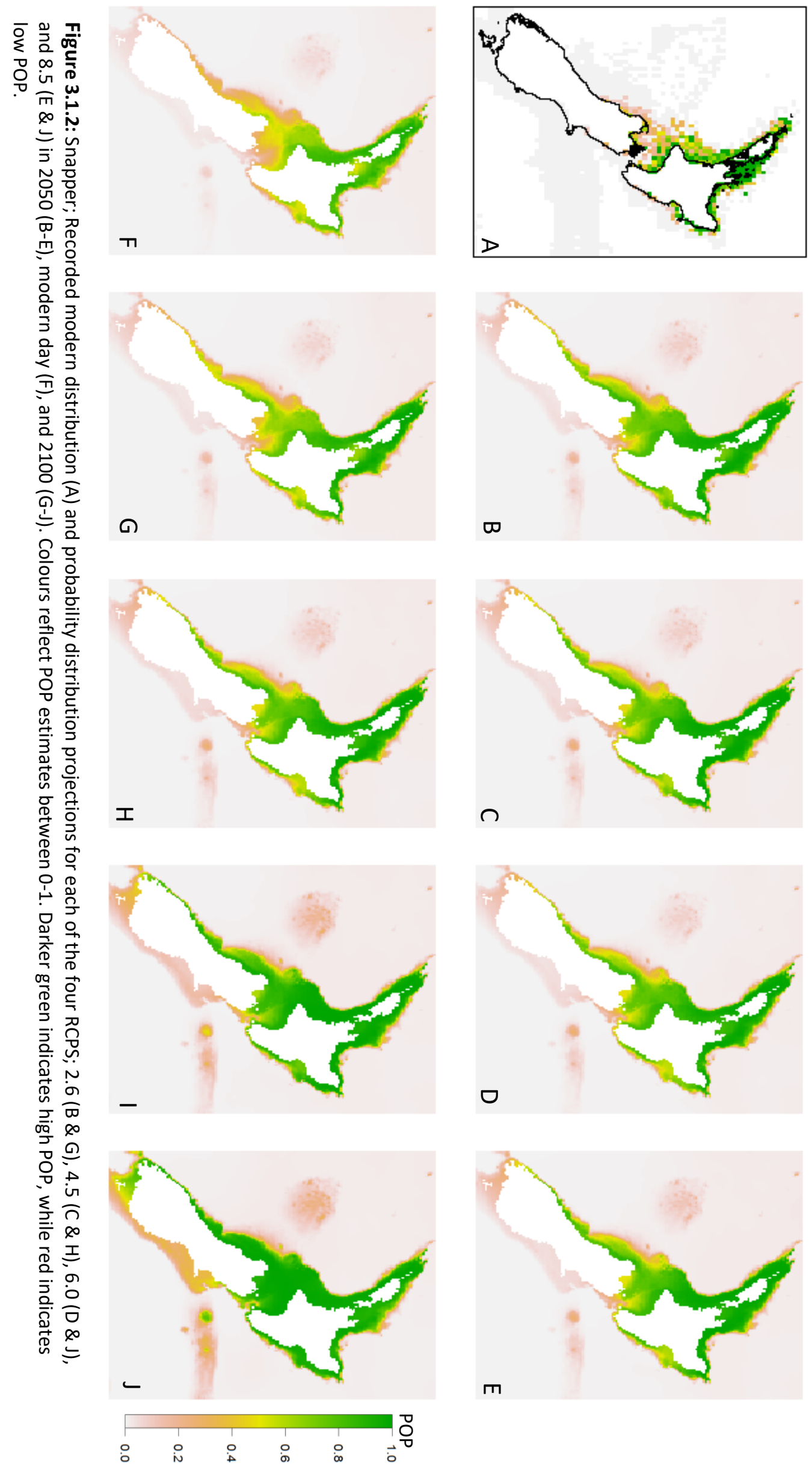


Table 3.1.2: Snapper; Change in POP values compared to the modern projection for each of the four climate scenarios 2050 and 2100 .

\begin{tabular}{ccccccccc}
\hline \multicolumn{9}{c}{$\mathbf{2 0 5 0}$} \\
POP & RCP2.6 & RCP4.5 & RCP6.0 & RCP8.5 & RCP2.6 & RCP4.5 & RCP6.0 & RCP8.5 \\
\hline $\mathbf{0 . 9}$ & 342.727 & 386.731 & 300.041 & 412.881 & 220.458 & 333.099 & 564.953 & 897.946 \\
$\mathbf{0 . 8}$ & 28.993 & 41.423 & 38.059 & 51.283 & 10.789 & 37.736 & 95.196 & 135.596 \\
$\mathbf{0 . 7}$ & 32.578 & 33.618 & 33.923 & 36.447 & 2.76 & 29.956 & 53.6 & 74.732 \\
$\mathbf{0 . 6}$ & 11.969 & 9.708 & 10.473 & 10.119 & 0.185 & 6.456 & 16.97 & 29.562 \\
$\mathbf{0 . 5}$ & -2.819 & -4.766 & -2.836 & -5.065 & -6.049 & -6.432 & -4.937 & 5.7 \\
$\mathbf{0 . 4}$ & -13.309 & -15.049 & -12.255 & -15.74 & -11.912 & -15.568 & -17.434 & -7.137 \\
$\mathbf{0 . 3}$ & -22.16 & -24.026 & -21.734 & -25.136 & -18.503 & -24.314 & -25.348 & -9.917 \\
$\mathbf{0 . 2}$ & -27.519 & -29.088 & -26.47 & -29.231 & -22.955 & -29.141 & -28.039 & -9.667 \\
$\mathbf{0 . 1}$ & -25.489 & -25.807 & -24.226 & -24.608 & -21.451 & -25.768 & -13.974 & -1.025 \\
\hline
\end{tabular}
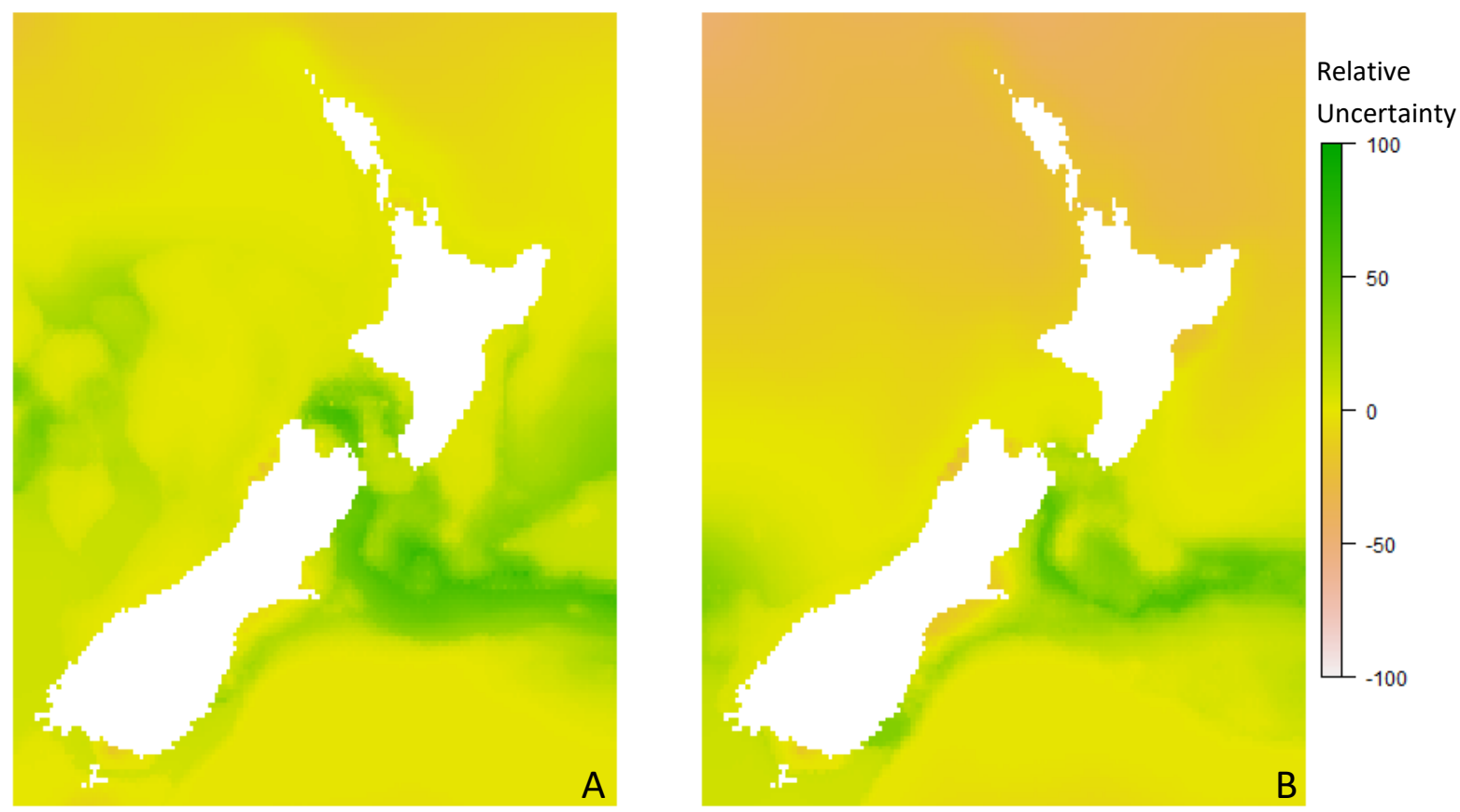

Figure 3.1.3: Snapper; MESS Maps of RCPS 2.6 2050 (A) and RCPS 8.52100 (B). MESS maps for all climate scenarios can be found in Appendix D.1. Negative sites indicate most relative uncertainty and positive sites indicate most relative certainty. 


\section{Variable Influence}

The base model predicted that snapper were virtually absent at depths below $-500 \mathrm{~m}$ and had increased POP with mean temperature (Figure 3.1.4). Information beyond 20 degrees was outside of the range of the data so a constant value was assumed and POP was fixed at 20 degrees at about 0.75. Temperature and salinity range had very little effect, and mean salinity did not contribute (Figure 3.1.4, Figure 3.1.5).

The limiting factor plots showed that the main climatic variable limiting contemporary snapper distribution was surface temperature (ST) mean from the mid NI southward (Figure 3.1.6A). In the RCPS 8.5100 limiting factor plot, this substantially shifted so that ST mean was the limiting factor in only the most south-eastern coastal regions of the SI (Figure 3.1.6B). Instead, the limiting climatic variable throughout most of coastal New Zealand in RCPS 8.5 was ST range.
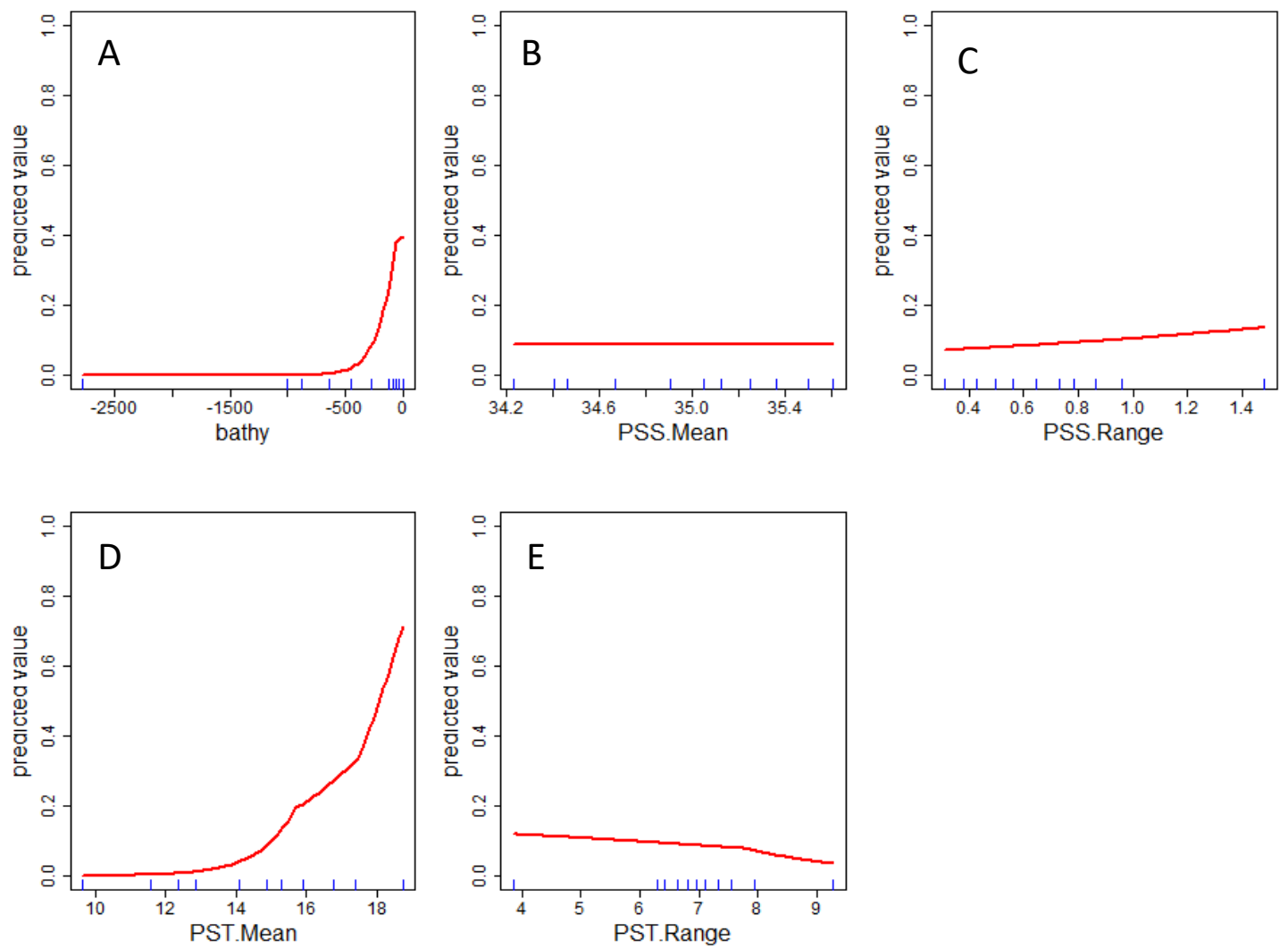

Figure 3.1.4: Snapper; Predictor response curves indicating how variables used for training affected the base model. Model predictions (red lines) and observations (blue data rug of deciles) are shown. The variables are bathymetry (A), surface salinity mean (B), surface salinity range (C), surface temperature mean (D), and surface temperature range $(E)$. 


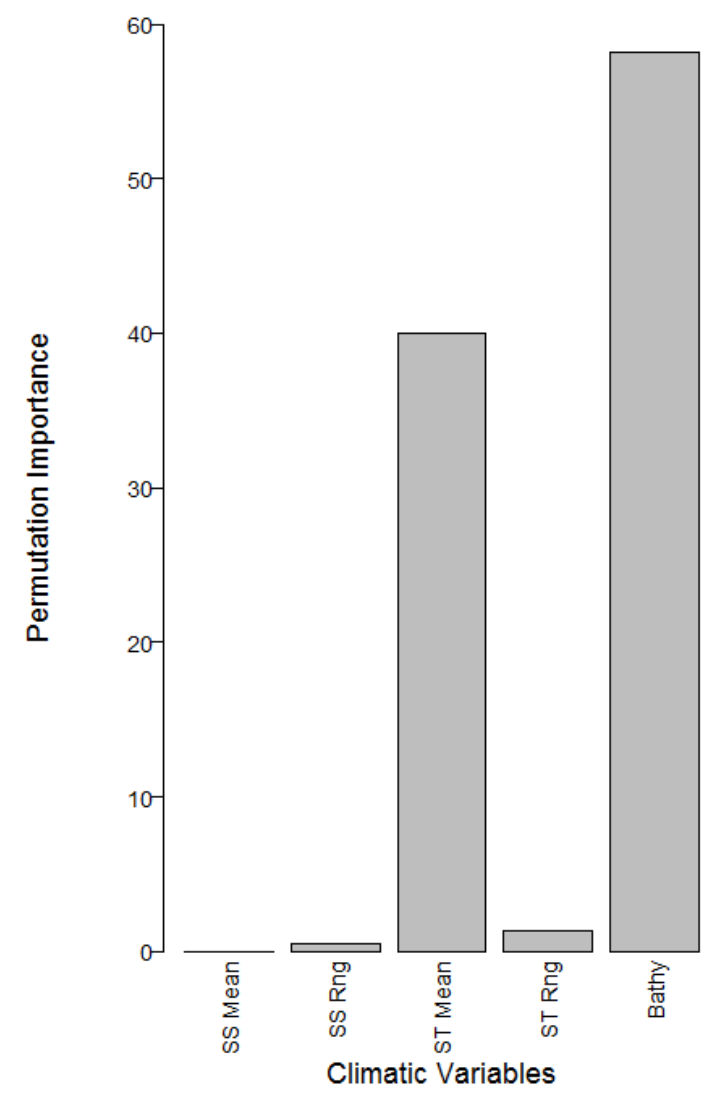

Figure 3.1.5: Snapper; Predictor variable contribution based on permutation importance (Phillips, 2006). Variable names are shortened here to fit: SS = Surface salinity, ST = Surface temperature, Rng = range, Bathy = bathymetry.
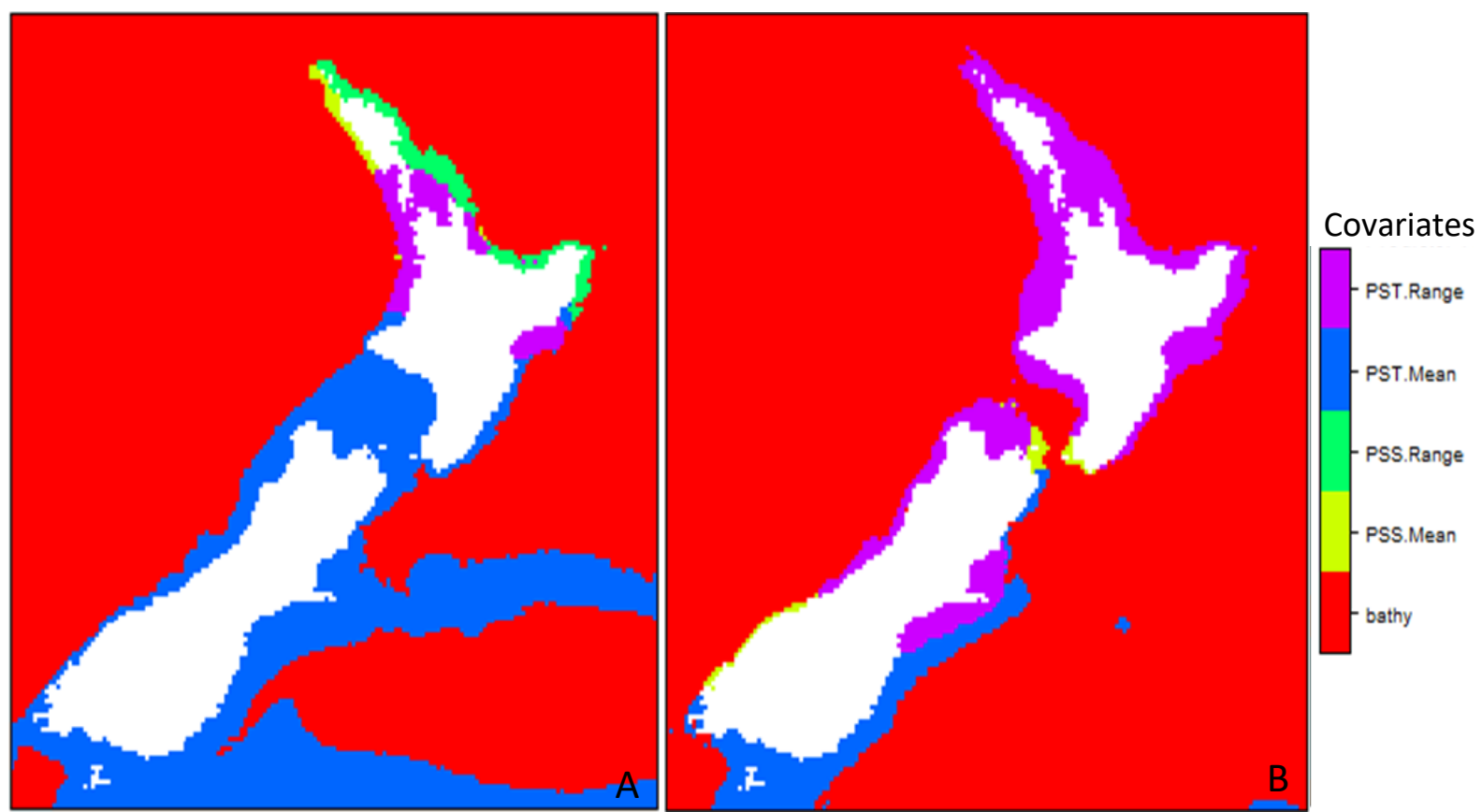

Figure 3.1.6: Snapper; Limiting factors plots of Modern (A), and RCPS 8.5 in 2100 (A) climates. Colours indicate the most limiting variable to distribution in that area. 


\section{2: JOHN DORY (Zeus faber)}

\section{$\underline{\text { Model Selection }}$}

Of all models generated, $\triangle \mathrm{AIC}$ values varied from 0 - 3505.71. Only models with a $\triangle \mathrm{AIC}$ of 0 were selected. Train AUC scores varied from 0.59 - 0.97 and test AUC varied from 0.56 - 0.97 . To select models with good discriminatory ability only those $>0.75$ were selected. To select models with low overfitting, average test $\mathrm{OR}_{10}$ values varied from $0.05-0.27$ but only those that were $<0.16$ were selected and average $A \cup C_{D I F F}$ varied from $0-0.135$ but only those $<0.05$ were selected. Only the models that met these criteria were considered for base model selection (Table 3.2.1). All selected models had high discriminatory ability (>0.9). When projected onto modern climate layers, four models appeared to under-represent contemporary john dory distribution (Figure 3.2.1; A, B, C \& F), while one over estimated this (Figure 3.2.1D). The remaining model 4e34 (Figure 3.2.1E), reflected the modern distribution well, and had comparatively low overfitting values, $\left(A \cup C_{D I F F}=0.019,0 R_{10}=\right.$ 0.129). Therefore, model $4 \mathrm{e} 34$ was selected as the base model for future predictions.

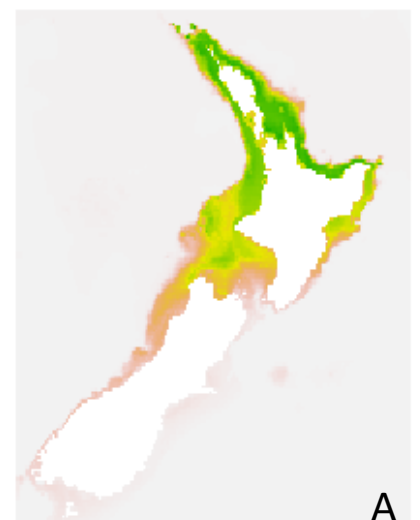

A

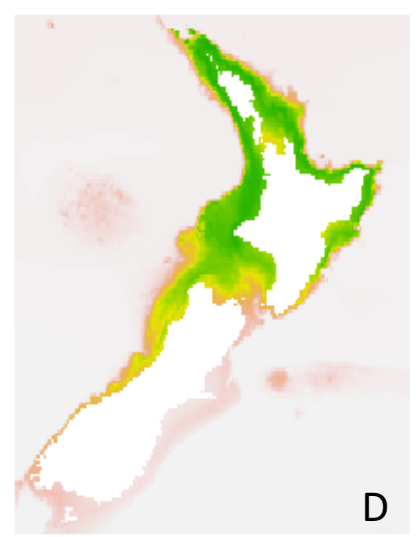

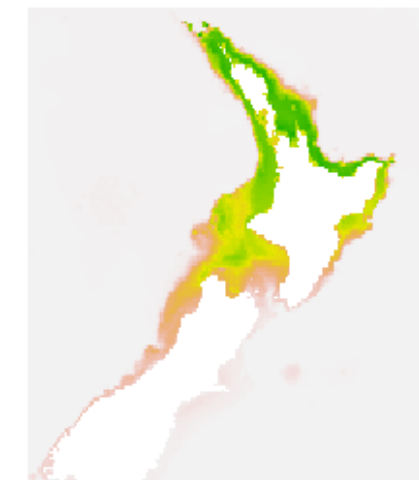

B

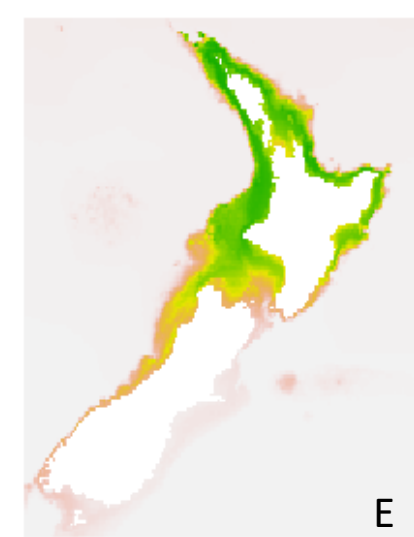

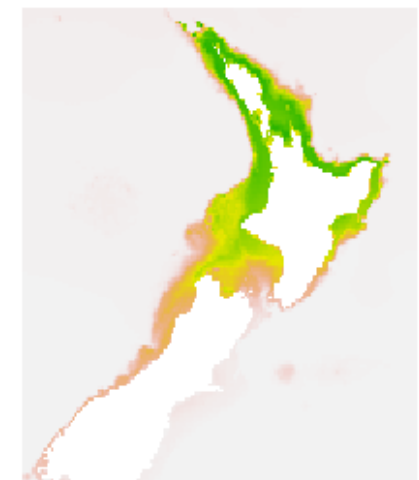

C

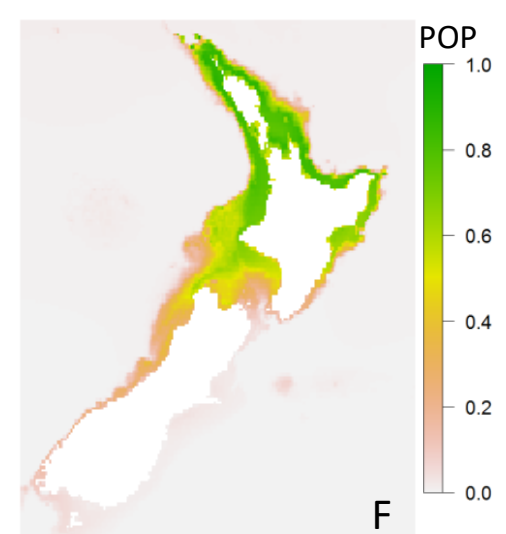

Figure 3.2.1: John dory; Six best Maxent models projected on modern climate layers; 1e5 (A), 1e6 (B), 2 e16 (C), $3 e 69(D), 4 e 34(E), 5 e 21(F)$. Colours reflect probability of presence estimates between $0-1$. Darker green indicates higher POP and red the reverse. 


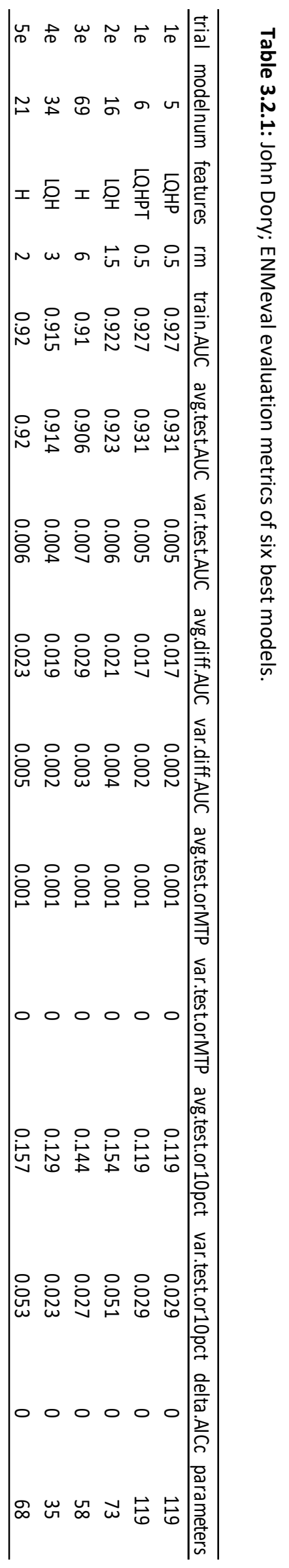




\section{Model Projections}

All 2050 RCPS projections were relatively similar (Figure 3.2.2B-E). All 2050 projections showed extension down the West Coast of the SI to roughly the same degree. POP consistently increased throughout the potential john dory range. In all climate scenarios, areas with highly preferable climatic conditions, (and therefore had the highest POP values), increased the most (POP $>0.9$ by $302.60-484.93 \%)$, while areas with lower POP increased much less or declined. The proportion of areas with POP values $<0.7$ decreased in all RCPS (Table 3.2.2).

The 2050 MESS maps for all RCPS displayed little relative uncertainty (Appendix D.2, Figure 3.2.3A). Most uncertainty was in the northern regions around the NI. In the more extreme RCPS 8.5, regions with relatively high uncertainty also included the Hauraki Gulf and Hawke Bay. Areas with particularly high relative certainty in most RCPS 2050 MESS maps were the Chatham Rise, through Cook Strait, to the West side of the SI.

\section{$\underline{2100 \text { Model Projections }}$}

Results for the 2100 predictions were more varied than the 2050 predictions, although similar patterns were observed (Figure 3.2G-J). There was again an increase in areas with high POP and decrease in areas with low POP. Areas with a POP $>0.9$ increased by $272.78-1126.02 \%$ and areas with a POP of $>0.5$ decreased by $3.34-19.93 \%$. Again, suitable conditions around the NI were maintained and POP generally increased in all predictions. Probable distribution extended further down the West Coast of the SI and reached Stewart Island in both RCPS 6.0 and 8.5. RCPS 8.5 also showed a substantial increase in POP on the east coast of the SI.

The 2100 MESS maps showed slightly more relative uncertainty, particularly in the most extreme scenario, RCPS 8.5 (Figure 3.2.3B, Appendix D.2). Most uncertainty was from the top of the $\mathrm{NI}$ to the middle of the $\mathrm{NI}$, including Hawke Bay. Other areas with relatively high uncertainty included the bays on either side of Banks Peninsular on the East Coast of the $\mathrm{SI}$, and around Karamea Bight at the north of the SI. 


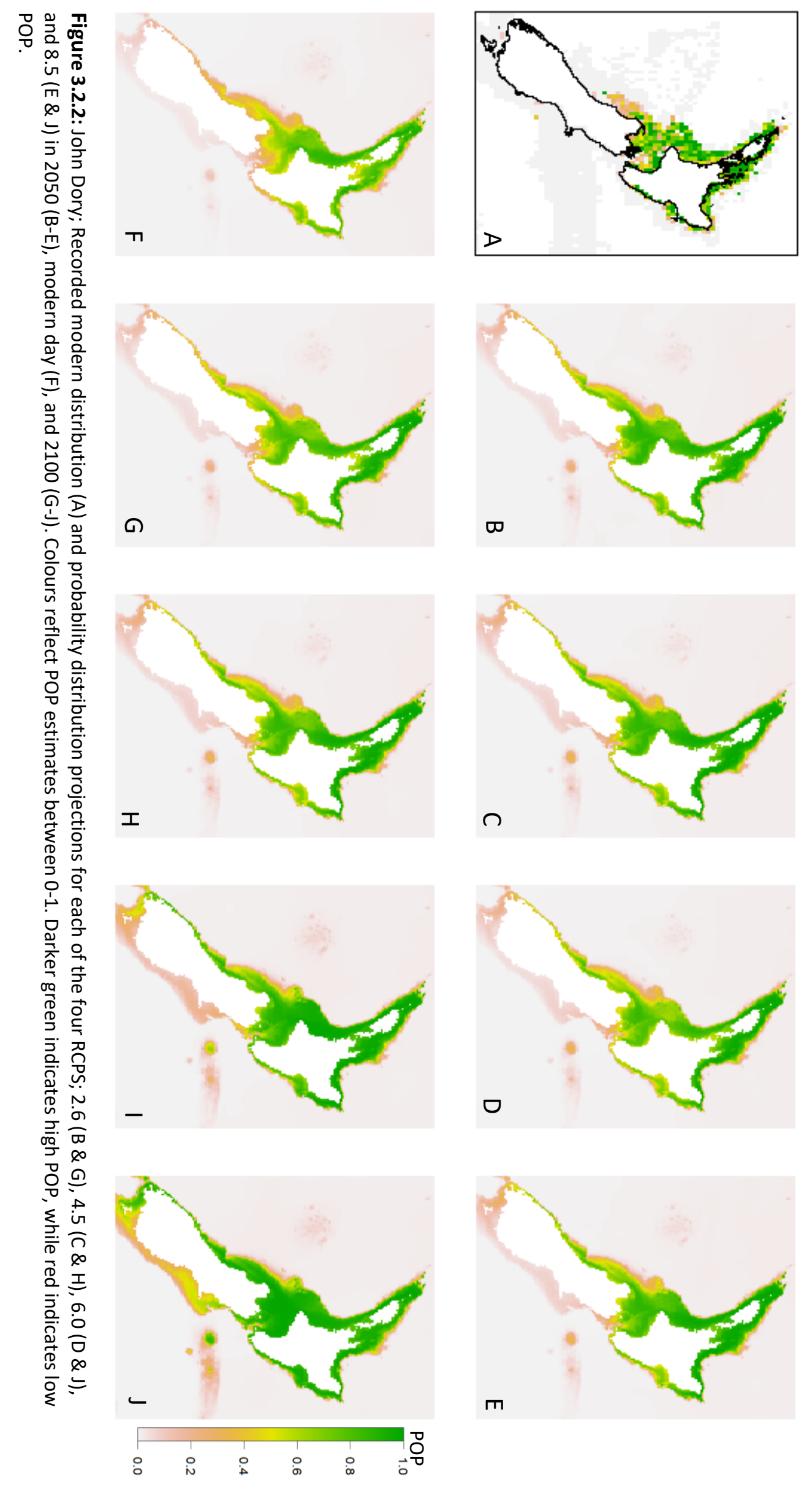


Table 3.2.2: John dory; Change in POP values compared to the modern projection for each of the four climate scenarios 2050 and 2100.

\begin{tabular}{lllllllll}
\hline \multicolumn{9}{c}{$\mathbf{2 0 5 0}$} \\
POP & RCP2.6 & RCP4.5 & RCP6.0 & RCP8.5 & RCP2.6 & RCP4.5 & RCP6.0 & RCP8.5 \\
\hline $\mathbf{0 . 9}$ & 340.203 & 474.433 & 302.599 & 484.93 & 272.781 & 437.77 & 796.408 & 1126.016 \\
$\mathbf{0 . 8}$ & 58.057 & 57.812 & 21.086 & 61.044 & 25.887 & 50.126 & 102.45 & 176.681 \\
$\mathbf{0 . 7}$ & 9.997 & 7.095 & -6.614 & 1.923 & -3.677 & 2.897 & 15.359 & 46.061 \\
$\mathbf{0 . 6}$ & -6.92 & -11.133 & -8.203 & -15.45 & -11.021 & -12.177 & -10.095 & 8.46 \\
$\mathbf{0 . 5}$ & -15.466 & -19.986 & -13.028 & -23.344 & -15.997 & -19.934 & -22.17 & -3.343 \\
$\mathbf{0 . 4}$ & -21.867 & -26.967 & -18.859 & -29.815 & -20.463 & -26.297 & -29.501 & -9.199 \\
$\mathbf{0 . 3}$ & -26.932 & -31.388 & -23.849 & -34.545 & -24.804 & -30.7 & -34.178 & -12.853 \\
$\mathbf{0 . 2}$ & -29.288 & -33.296 & -25.618 & -35.869 & -26.457 & -32.388 & -35.04 & -15.585 \\
$\mathbf{0 . 1}$ & -28.527 & -32.571 & -24.374 & -34.452 & -26.009 & -31.465 & -27.377 & -15.599 \\
\hline
\end{tabular}
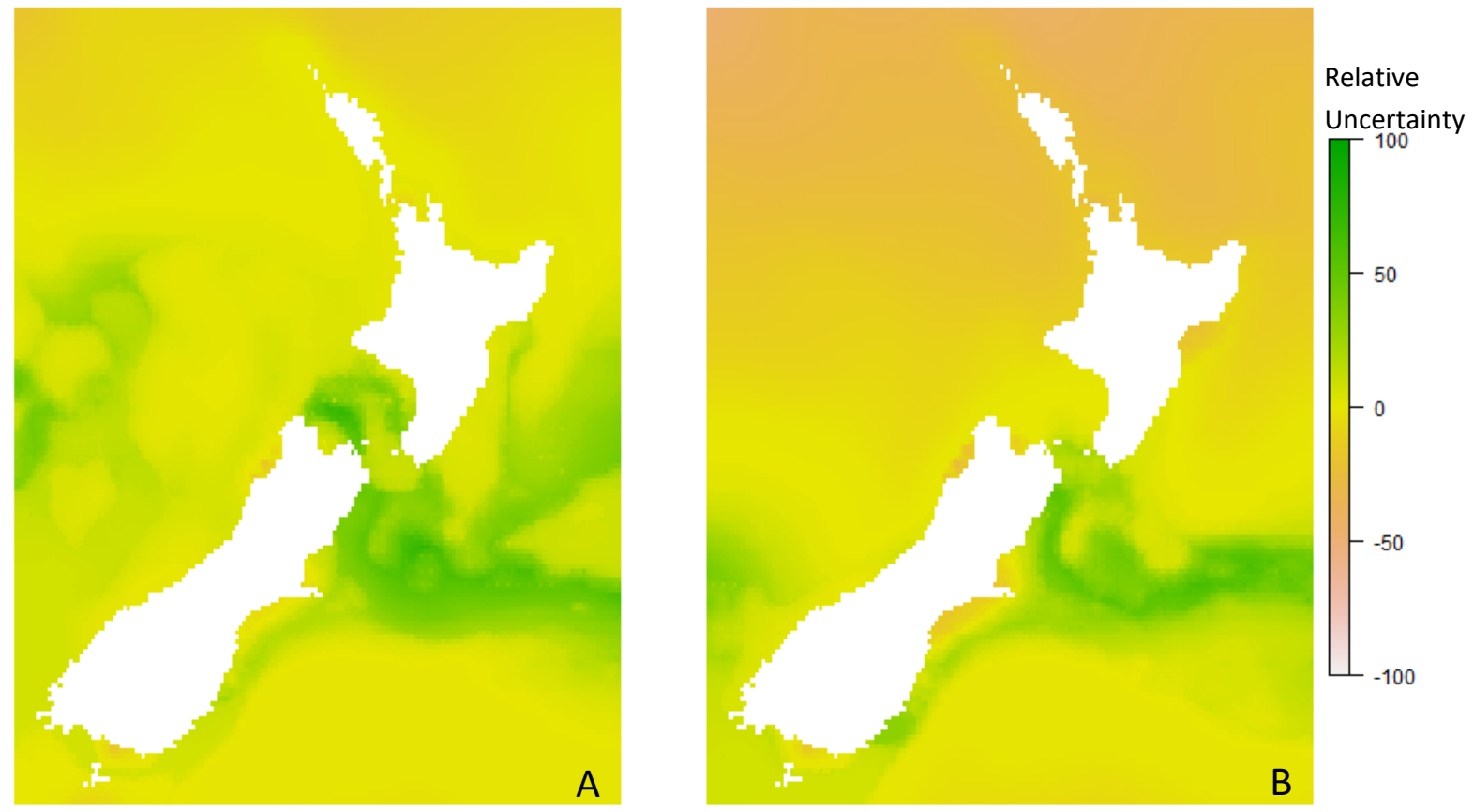

Figure 3.2.3: John dory; MESS Maps of RCPS 2.62050 (A) and RCPS 8.52100 (B). MESS maps for all climate scenarios can be found in Appendix D.2. Negative sites indicate most relative uncertainty and positive sites indicate most relative certainty. 


\section{Variable Influence}

The base model predicted john dory were absent below -500m and had increased POP with mean temperature (Figure 3.4). Information beyond 19 degrees was outside of the range of data used to train the model so a constant value was assumed and POP was fixed at 19 degrees at about 0.95. ST mean contributed most to the model, other climatic variables had comparatively little contribution (Figure 3.4 and 3.5).

The modern liming factor plot (Figure 3.6A), showed ST mean was main the limiting climatic variable for contemporary john dory distribution, particularly in regions south of the mid NI. In the future limiting factor plot the area limited by ST mean was substantially reduced and confined to the lower eastern SI regions (Figure 3.6B). Instead, the limiting climatic variable in regions north of the mid SI and the majority of the future limiting factor plot was ST range.
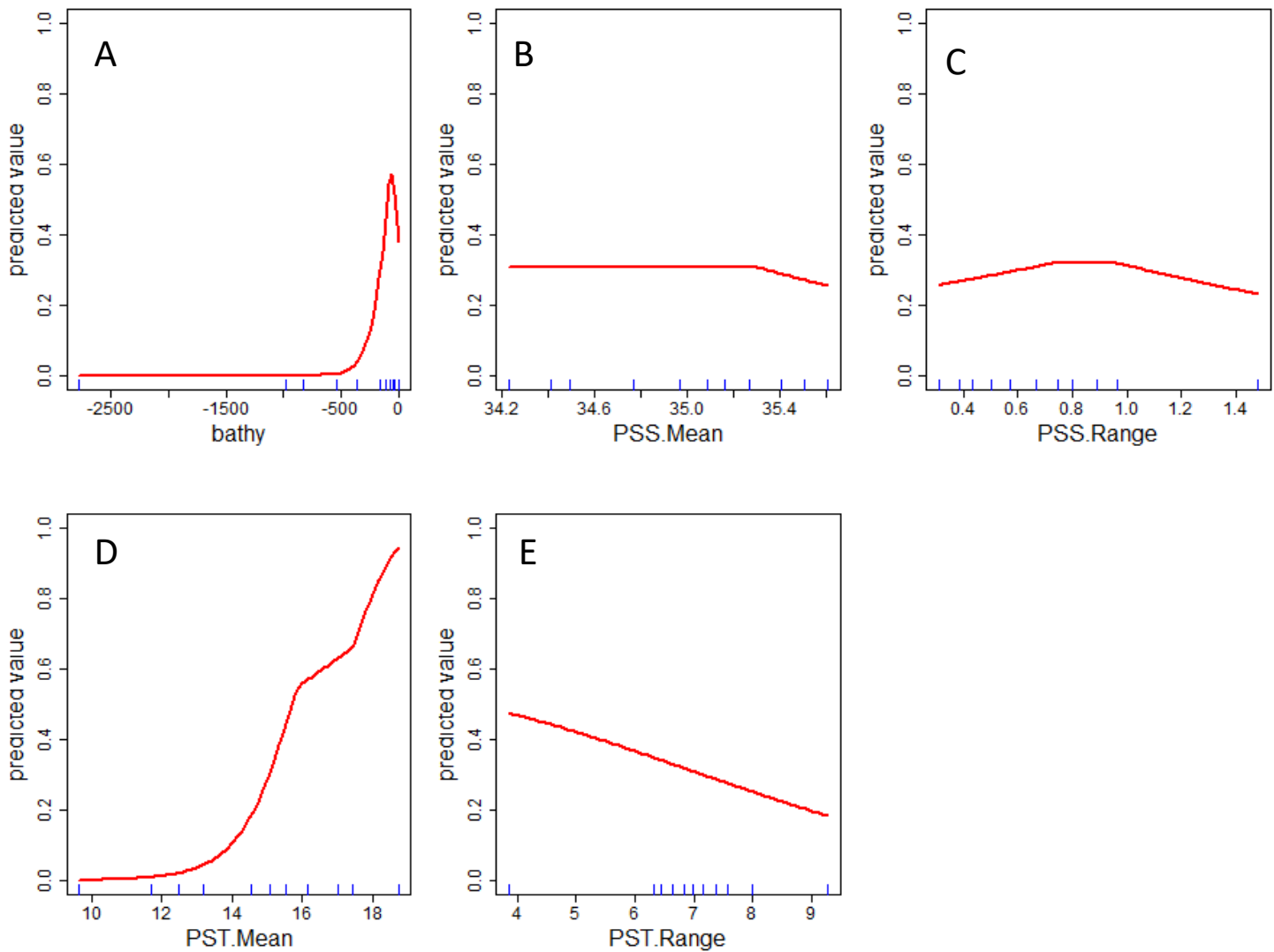

Figure 3.2.4: John dory; Predictor response curves indicating how variables used for training affected the base model. Model predictions (red lines) and observations (blue data rug of deciles) are shown. The variables are bathymetry $(A)$, surface salinity mean $(B)$, surface salinity range $(C)$, surface temperature mean $(D)$, and surface temperature range $(\mathrm{E})$. 


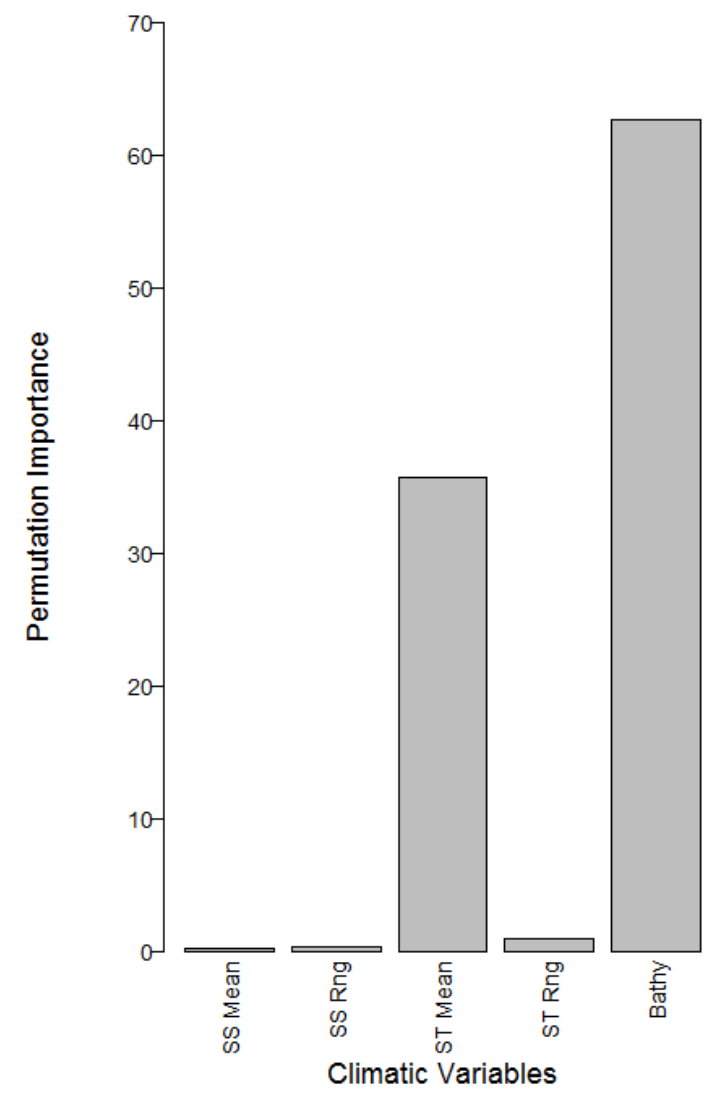

Figure 3.2.5: John dory; Predictor variable contribution based on permutation importance (Phillips, 2006).

Variable names are shortened here to fit: SS = Surface salinity, ST = Surface temperature, Rng = range, Bathy = bathymetry.
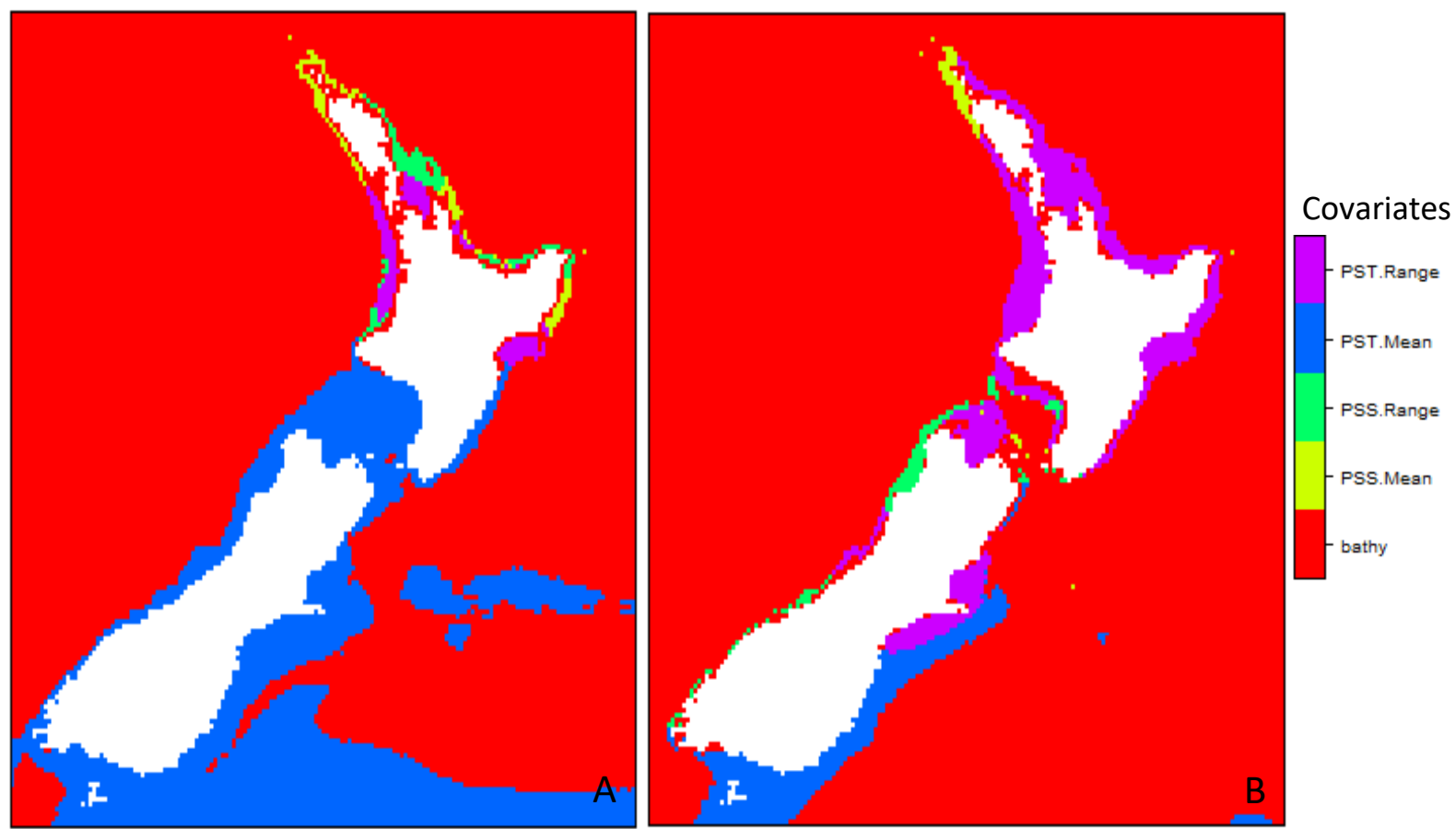

Figure 3.2.6: John dory; Limiting factors plots of Modern (A), and RCPS 8.5 in 2100 (A) climates. Colours indicate the most limiting variable to distribution in that area. 


\section{3: TREVALLY (Pseudocaranx georgianus)}

\section{$\underline{\text { Model Selection }}$}

Models selected are shown in table 3.1. $\triangle$ AIC values varied form $0-1482.2$, only models with a $\triangle A I C$ of 0 were selected. Train AUC scores varied from 0.59 - 0.98 and test AUC from $0.62-0.98$. To select models with good discriminatory ability only those $>0.75$ were selected. To select models with low overfitting average test $\mathrm{OR}_{10}$ values varied from $0.036-0.19$ but only those that were $<0.11$ were selected and $A \cup C_{D I F F}$ varied from $0.001-0.079$ but only those $<0.05$ were selected. When projected, the selected models each matched the contemporary distribution relatively well (Figure 3.3.1) although, models $2 \mathrm{~d} 16$ (Figure 3.3.1B), and $5 \mathrm{~d} 10$ (Figure 3.3.1E) underestimated distribution in the southern NI and slightly overestimated around the Hauraki Gulf. Unfortunately, these two models were the only ones where climatic variables had any influence. Selecting models where an environmental response was detected rather than those where it was not (models 1e28, 2e27, 4e2, 5e27) did not require a substantial trade-off in model quality as all six selected best models high discriminatory ability $\left(A \cup C_{\text {TRAIN }}\right.$ and $A U C_{\text {TEST }}$ both $>0.9$ ) and low overfitting, with $\mathrm{OR}_{10}$ values $\left(\mathrm{OR}_{10}=\right.$ 0.076 - 0.107 ) barely exceeding the $10 \% \mathrm{OR}_{10}$ threshold recommended by Muscarella et al. (2014) (Table 3.3.1). Model $2 \mathrm{~d} 16$ had slightly lower overfitting metrics ( $A \cup C_{\mathrm{DIFF}}=0.012, \mathrm{OR}_{10}=0.09$ ) (Table 3.3.1). Model $2 \mathrm{~d} 16$ was therefore selected as the base model. 


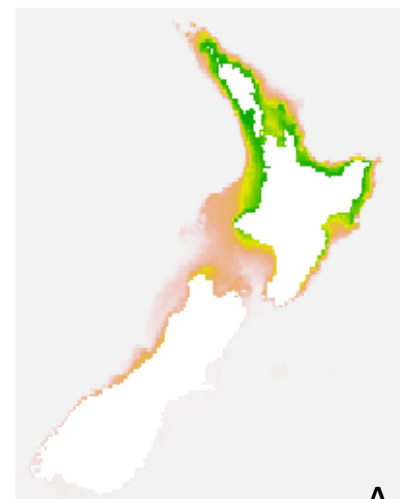

A

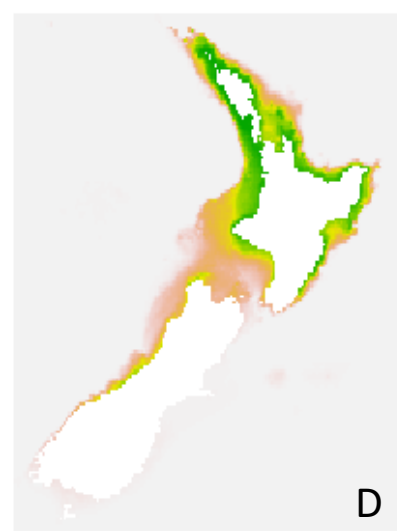

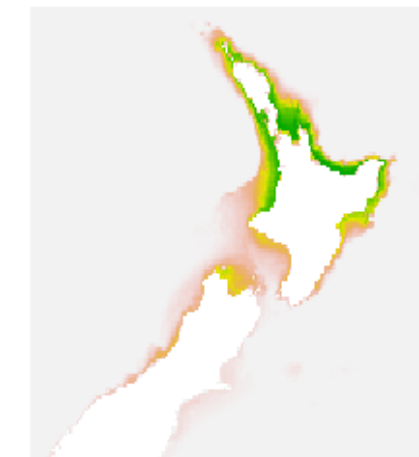

B

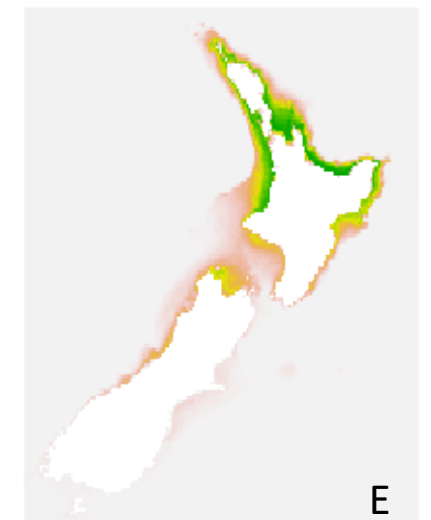

C

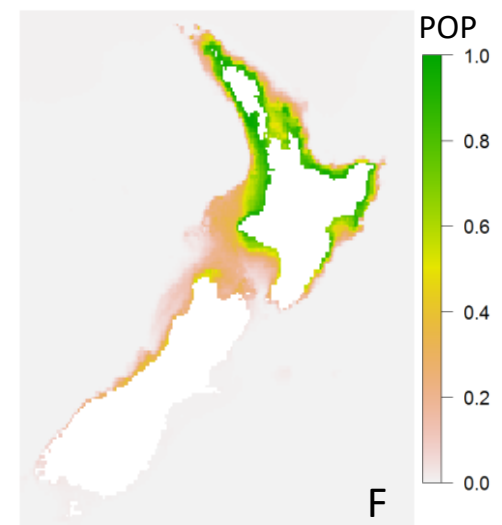

Figure 3.3.1: Trevally; Six best Maxent models projected on modern climate layers; $1 \mathrm{e} 28$ (A), $2 \mathrm{~d} 16$ (B), $2 \mathrm{e} 27$ (C), 4e2 (D), $5 \mathrm{~d} 10$ (E), $5 \mathrm{e} 27$ (F). Colours reflect probability of presence estimates between 0-1. Darker green indicates higher POP and red the reverse. 


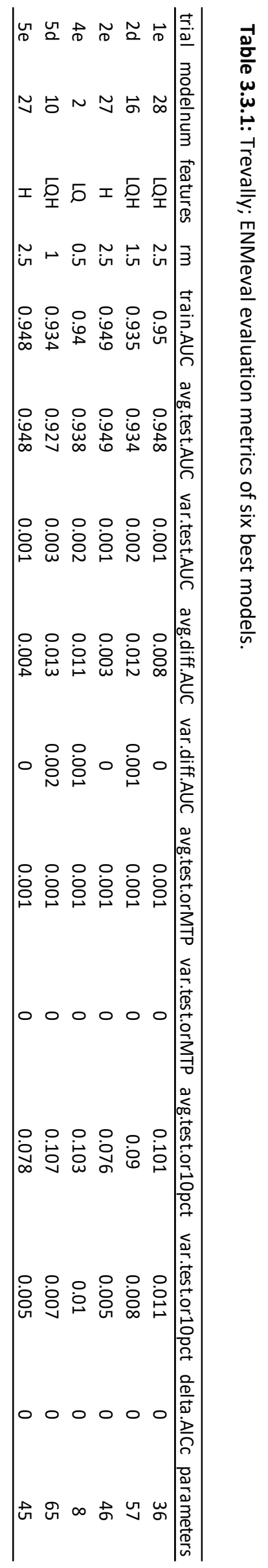




\section{Model Projections}

Each of the 2050 RCPS predictions suggested southward shifts for trevally distributions (Figure 3.3.2B-E). Shift extents were more substantial the more the climate scenario deviated from the contemporary conditions. RCPS 8.5 had the most notable changes from the contemporary prediction. POP in the northern regions decreased from $>0.8$ to $<0.4$ in some areas, while POP increased equivalently around the southern $\mathrm{NI}$ and top of the SI. In RCPS 4.5, 6.0 and 8.5 a slight increase in POP down the west coast of the SI was also visible. In all RCPS, except RCPS 6.0 areas with highly preferable climatic conditions, and therefore had the highest POP values, decreased the most, while areas with lower POP decreased much less or increased. The proportion of area occupied by a POP value of $>0.9$ decreased by $22.29-51.00 \%$, and in the case of RCPS 6.0 increased by $1.01 \%$. Areas with a POP value of $>0.5$ decreased by $6.98-$ $24.38 \%$, and in the case of RCPS 6.0 increased by $1.88 \%$ (Table 3.3.2).

The 2050 MESS maps for each RCPS looked similar, with most relative uncertainty in the southern region at the bottom of the $\mathrm{SI}$ (Figure 3.3.3A, Appendix D.3). Areas with particularly high certainty were regions on the eastern side of the $\mathrm{NI}$ and in the Cook Strait.

\section{$\underline{2100 \text { Model Projections }}$}

2100 RCPS projections had considerably more variation than 2050 projections (Figure 3.3.2G-J). The areas with suitable conditions in the RCPS 2.62100 projection appeared very similar to the 2050 projection. Despite this, decrease in areas with high POP was not as dramatic so not as much quality suitable habitat was lost in the 2100 prediction as it was in the 2050 prediction. In 2050 POP between 0.8 and 0.9 decreased by $45 \%$, while areas with the same POP only decreased by $12.74 \%$ in the 2100 prediction (Table 3.3.2). This was the case for all RCPS in 2100, except for RCPS 8.5, where areas with high POP were dramatically reduced. In this scenario, areas with a POP of $>0.9$ decreased by $91.05 \%$. The RCPS 4.5 and RCPS 6.02100 projections showed similar but more substantial southwards shifts of suitable conditions to the 2050 predictions. In RCPS 6.0 and 8.5, POP was more substantially reduced in the top half of the NI. Like the 2050 predictions, in RCPS 4.5 and to a greater extent in RCPS 8.5, highly preferable conditions were reduced more than average or low quality conditions. Hawke Bay was the only area that has a consistently high POP (>0.7) in all predictions, except RCPS 8.52100.

The 2100 MESS maps showed similar relative uncertainty patterns to the 2050 MESS maps (Appendix D.3), with the exception of the most extreme scenario RCPS 8.5 (Figure 3.3.3B). This showed considerably more uncertainty from the top of the NI to the middle of the NI, including Hawke Bay. 


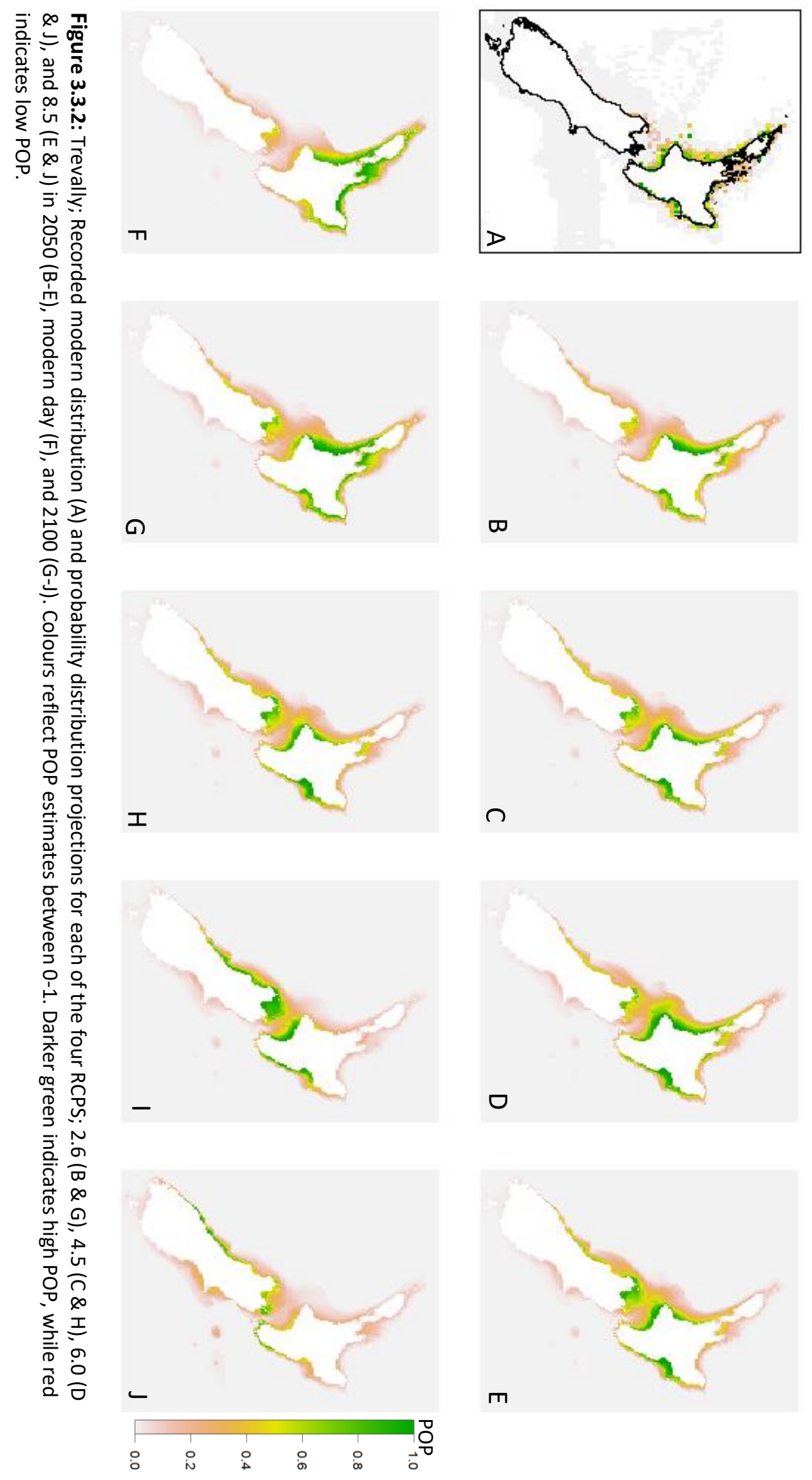


Table 3.3.2: Trevally; Change in POP values compared to the modern projection for each of the four climate scenarios 2050 and 2100.

\begin{tabular}{lllllllll}
\hline \multicolumn{7}{c}{$\mathbf{2 0 5 0}$} \\
POP & RCP2.6 & RCP4.5 & RCP6.0 & RCP8.5 & RCP2.6 & RCP4.5 & RCP6.0 & RCP8.5 \\
\hline $\mathbf{0 . 9}$ & -41.943 & -51.001 & 1.013 & -22.288 & -3.143 & -40.115 & 4.298 & -91.052 \\
$\mathbf{0 . 8}$ & -44.998 & -36.939 & -3.868 & -24.604 & -12.739 & -33.072 & 9.787 & -82.198 \\
$\mathbf{0 . 7}$ & -40.499 & -35.329 & -15.621 & -26.734 & -8.806 & -35.655 & -5.539 & -78.516 \\
$\mathbf{0 . 6}$ & -37.093 & -25.507 & -7.457 & -18.53 & 1.386 & -27.512 & -10.825 & -72.788 \\
$\mathbf{0 . 5}$ & -24.382 & -15.355 & 1.879 & -6.983 & 9.457 & -15.624 & -16.094 & -68.715 \\
$\mathbf{0 . 4}$ & -16.023 & -7.849 & 11.777 & -4.141 & 12.758 & -9.896 & -16.541 & -63.935 \\
$\mathbf{0 . 3}$ & -10.517 & -0.822 & 16.636 & -0.154 & 14.865 & -3.393 & -18.535 & -55.468 \\
$\mathbf{0 . 2}$ & -2.746 & 6.922 & 18.347 & 5.308 & 21.186 & 4.381 & -20.396 & -41.216 \\
$\mathbf{0 . 1}$ & 2.208 & 9.64 & 12.927 & 7.107 & 13.942 & 8.862 & -15.676 & -18.163 \\
\hline
\end{tabular}
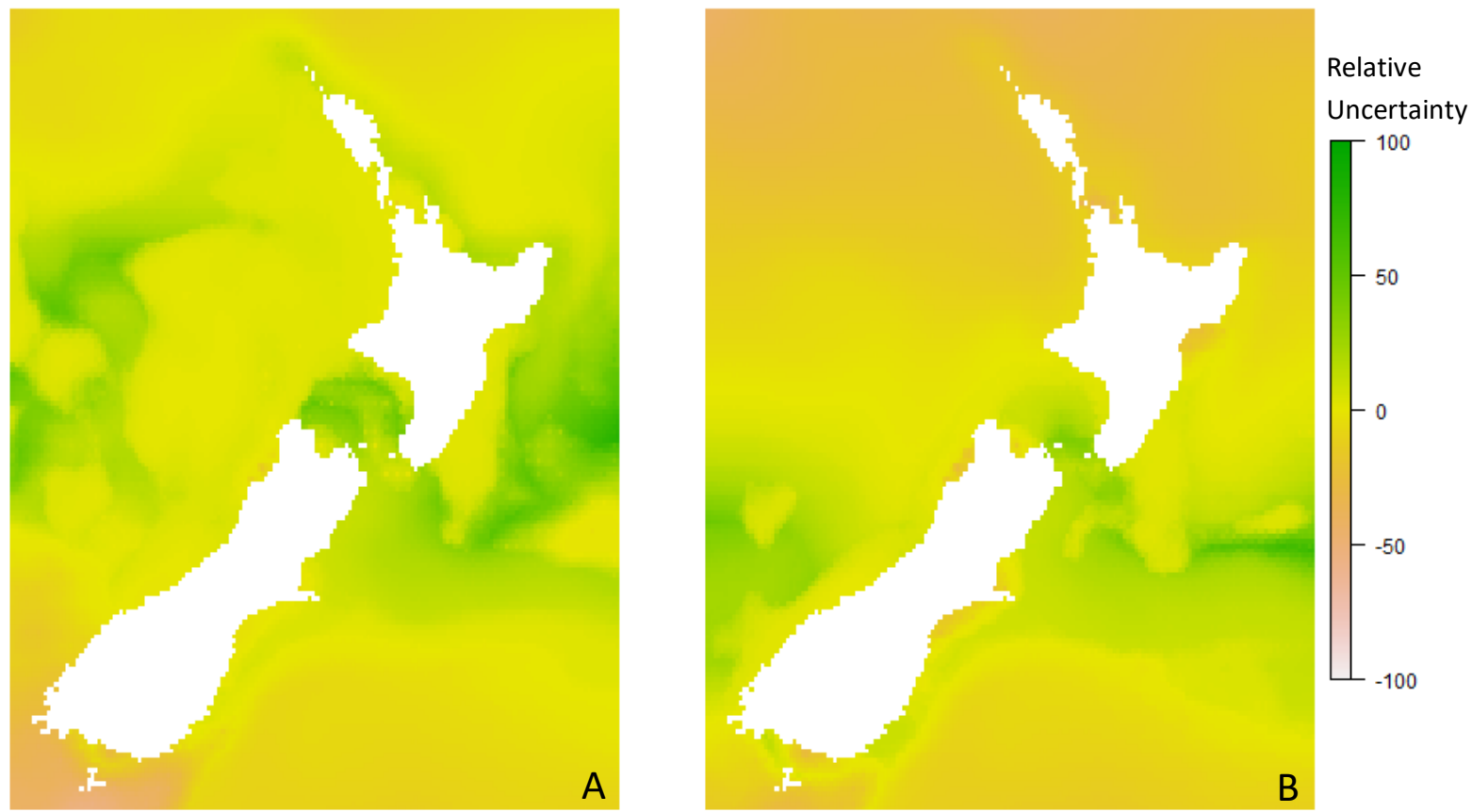

Figure 3.3.3: Trevally; MESS Maps of RCPS 2.62050 (A) and RCPS 8.52100 (B). MESS maps for all climate scenarios can be found in Appendix D.3. Negative sites indicate most relative uncertainty and positive sites indicate most relative certainty. 


\section{Variable Influence}

The base model predicted trevally were absent below $-500 \mathrm{~m}$ and had slightly increased POP at mean temperatures between 16-18 degrees and at mean salinity between 34.8 - 35.6psu (Figure 3.3.4). Salinity range had no clear effect and temperature range slightly increased POP. All climatic variables had limited contribution to the model, however mean temperature contributed the most of out of these (Figure 3.3.5).

The modern liming factor plot (Figure 3.3.6A), showed ST mean was main the climatic limiter for contemporary trevally distribution, particularly in coastal regions south of the mid NI. The future limiting factor plot changed considerably so that the for coastal regions around the NI, Cook Strait, and half way down the west coast of the SI, were are mainly limited by ST mean (Figure 3.3.6B). Furthermore, the climatic variable limiting distribution on the east coast and southern New Zealand was mainly SS mean, and ST range around the S/W corner.
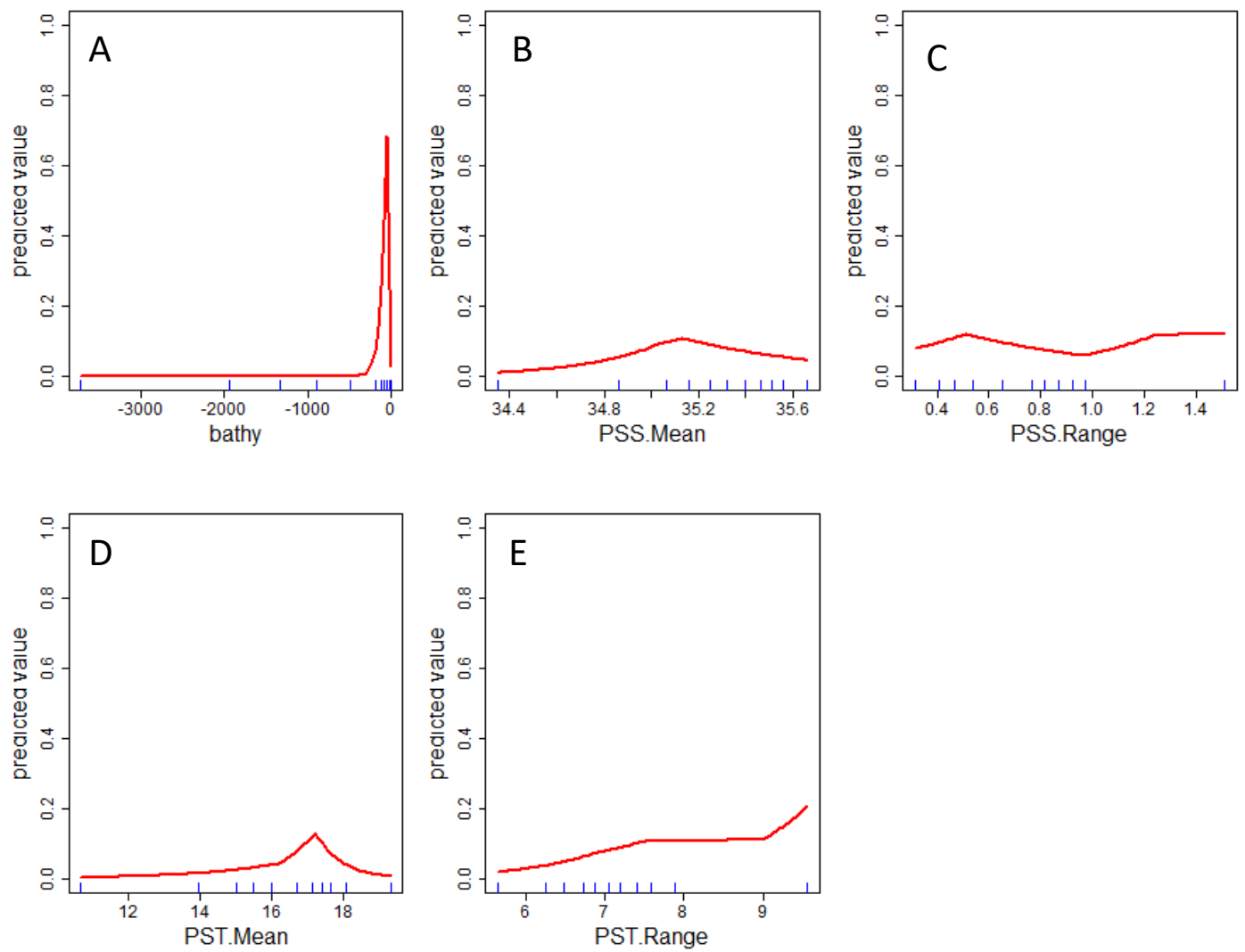

Figure 3.3.4: Trevally; Predictor response curves indicating how variables used for training affected the base model. Model predictions (red lines) and observations (blue data rug of deciles) are shown. The variables are bathymetry $(A)$, surface salinity mean $(B)$, surface salinity range $(C)$, surface temperature mean $(D)$, and surface temperature range $(\mathrm{E})$. 


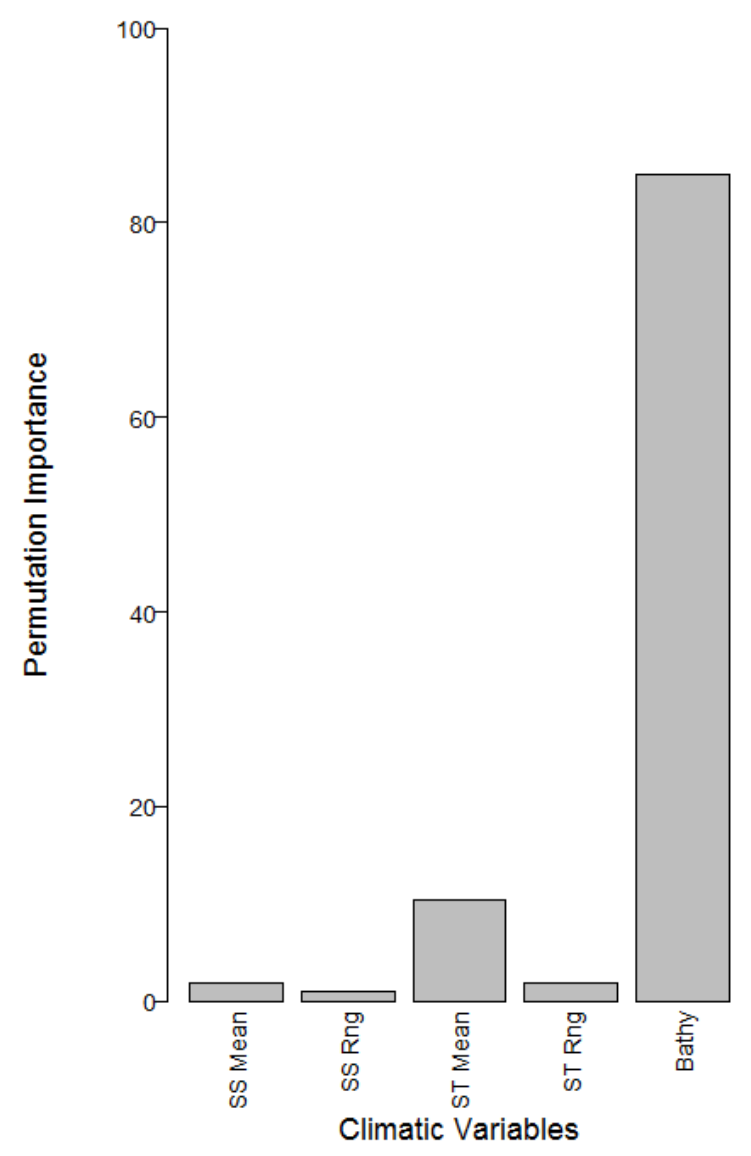

Figure 3.3.5: Trevally; Predictor variable contribution based on permutation importance (Phillips, 2006).

Variable names are shortened here to fit: SS = Surface salinity, ST = Surface temperature, Rng = range, Bathy = bathymetry.

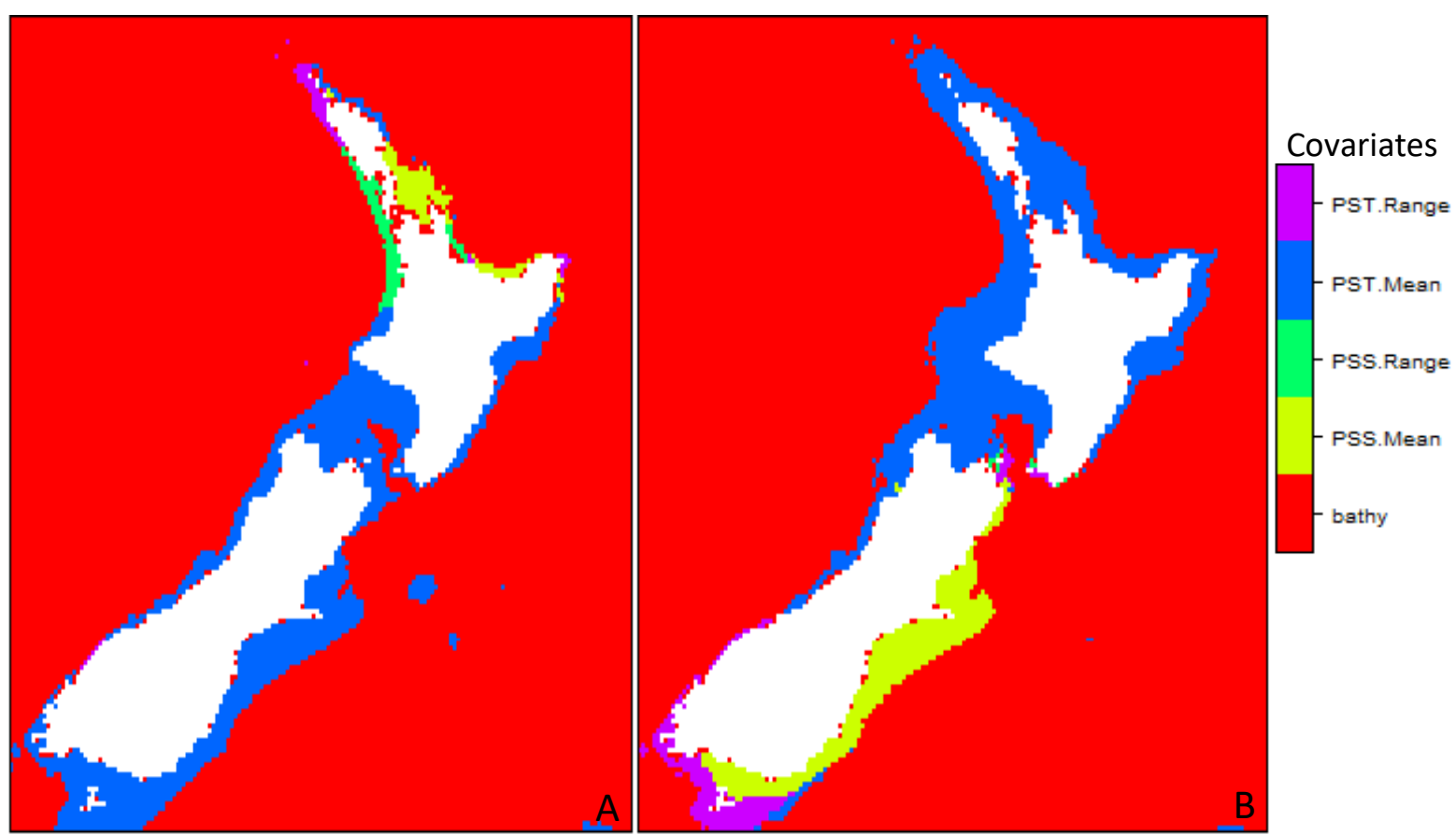

Figure 3.3.6: Trevally; Limiting factors plots of Modern (A), and RCPS 8.5 in 2100 (A) climates. Colours indicate the most limiting variable to distribution in that area. 


\section{4: TARAKIHI (Nemadactylus macropterus)}

\section{$\underline{\text { Model Selection }}$}

Models selected are shown in table 3.4.1. $\triangle$ AIC values varied form 0 - 12671.95. Only models with a $\triangle A I C$ of 0 were selected. Train AUC scores varied from $0.57-0.97$ and test AUC from $0.58-0.97$. To select models with good discriminatory ability only those $>0.75$ were selected. To select models with low overfitting average test $\mathrm{OR}_{10}$ values varied from $0.04-0.27$ but only those that were $<0.13$ were selected and average $A \cup C_{D I F F}$ varied from $0-0.09$ but only those $<0.05$ were selected. When selected models that fit these criteria were projected onto modern climates, there was notable variation between the probability distributions (Figure 3.4.1). All models somewhat correctly predicted the contemporary populations around Cook Strait and the upper SI, and the east coast of the $\mathrm{SI}$ but their predictions around the upper $\mathrm{NI}$, west coast of the $\mathrm{NI}$ and lower SI varied considerably. Models $3 \mathrm{c} 4$ and $3 \mathrm{~d} 9$ (Figure 3.4.1B\&C) appeared to resemble tarakihi distribution slightly better than the other projections, particularly in the furthermost northern and southern regions. Model 3d9 had higher discriminatory ability than $3 c 4\left(A \cup C_{\text {TRAIN }} \& A U C_{\text {TEST }}=0.88\right)$ and lower overfitting than $3 c 4$ and all other models $\left(A \cup C_{D I F F}=0.007, \mathrm{OR}_{10}=0.08\right)$ (Table 3.4.1). Model $3 \mathrm{~d} 9$ was therefore chosen as the base model for future projections. 


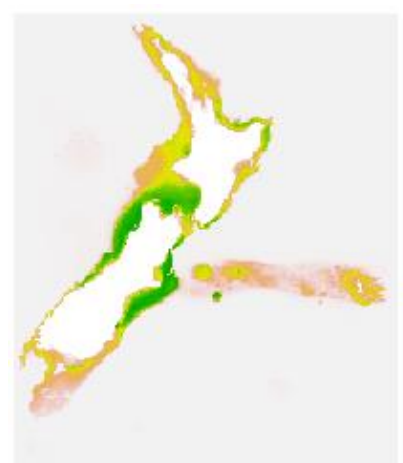

A

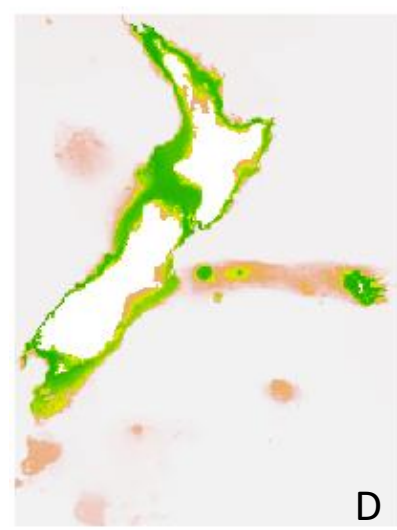

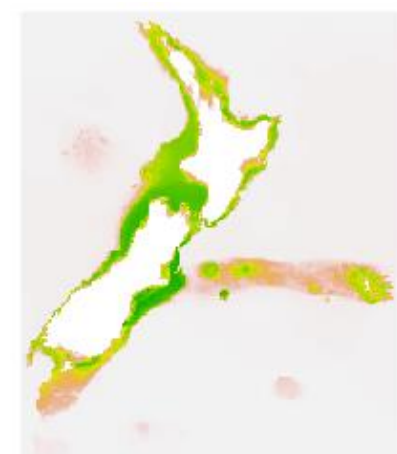

B

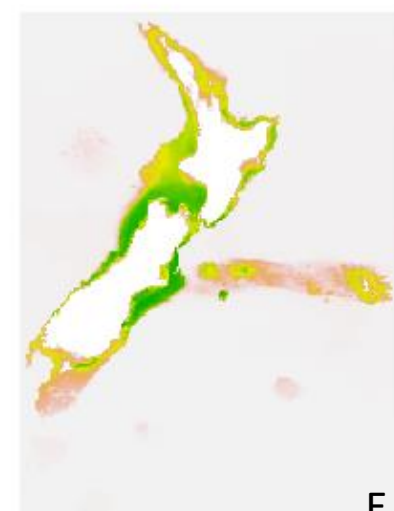

E

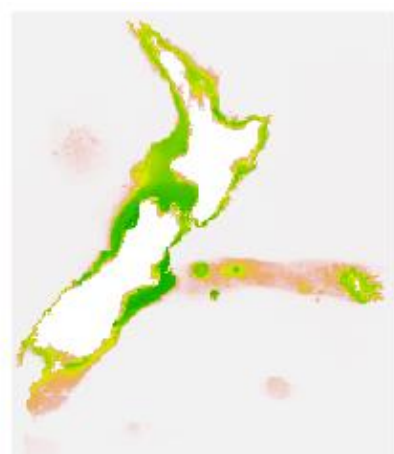

C

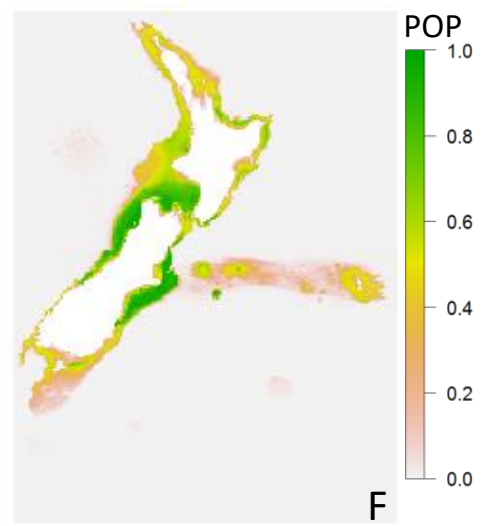

Figure 3.4.1: Tarakihi; Six best Maxent models projected on modern climate layers; $1 d 5$ (A), 3c4 (B), 3d9 (C), $3 e 69(D), 4 c 3(E), 4 d 5(F)$. Colours reflect probability of presence estimates between 0-1. Darker green indicates higher POP and red the reverse. 


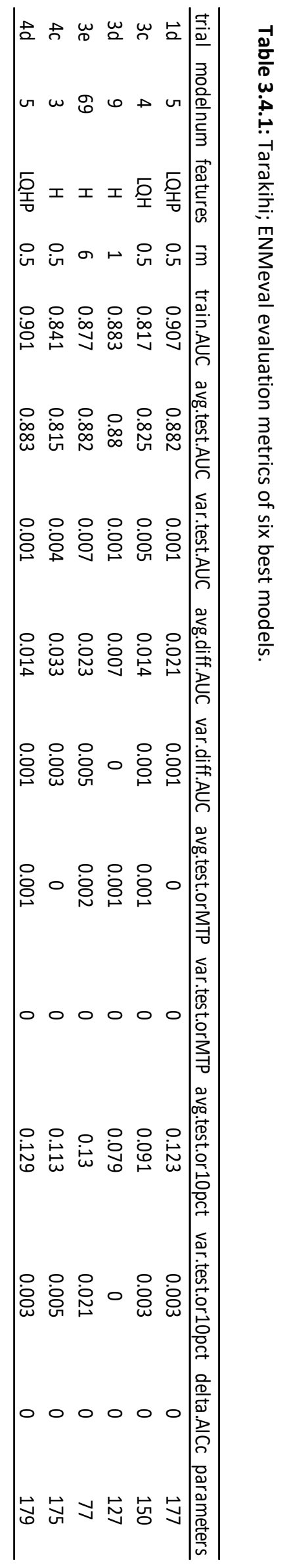




\section{Model Projections}

The 2050 tarakihi predictions generally showed an increase in POP in most regions, with the exception of the northern $\mathrm{NI}$ regions (Figure 3.4.2B-E). In all climate scenarios high POP areas contracted southward from the top of the NI. This was most substantial in the RCPS 8.5 prediction. In RCPS 4.5, 6.0 and 8.5, the potential distribution also extended further southward, past the bottom of the SI and Stewart Island. In all RCPS, Banks Peninsular and the mid-east coast of the SI, along with Cook Strait and the top to middle of the SI West Coast, remained stable areas with high POP. Overall areas with a POP of $>0.9$ increased by $77.54-311.16 \%$ while all areas with a POP of $>0.5$ decreased slightly or stayed the same (Table 3.4.2).

All four RCPS 2050 MESS maps looked similar and showed little relative uncertainty (Appendix D.4, Figure 3.4.3A). Relative certainty was highest around the South-Western areas of New Zealand, in the Cook Strait, and along the Chatham Rise, indicating these were areas where the predicted 2050 conditions most closely represented contemporary climate for the base model.

\section{Model Projections}

The 2100 predictions reflected similar patterns to the 2050 predictions in each RCPS but effects were more pronounced in RCPS 4.5, 6.0 and 8.5 (Figure 3.4.2G-J). These RCPS displayed further southward contraction of high POP from the NI. Areas with high POP receded from the NI almost entirely in the most extreme RCPS 8.5. POP increased around the Chatham Islands in scenarios 4.5, 6.0 and 8.5. Overall areas with a POP of $>0.9$ increased by $82.54-167.25 \%$. In RCPS 6.0 this increase was much less than the $311 \%$ increase in the 2050 prediction. Areas with a POP of $>0.5$ decreased by 6.49 $18.22 \%$ (with the exception of the RCPS 2.6 which increased by $0.28 \%$ ). As was with the 2050 predictions, area with highly preferable conditions for tarakihi increased, while area with less preferable conditions decreased.

The MESS maps also showed more variation in 2100 than 2050, with the RCPS 8.5 showing a greater amount of relative uncertainty, while the other MESS maps showed a similar uncertainty patterns as the 2050 MESS maps (Appendix D.4). Most uncertainty was in the RCPS 8.5 scenario around the northern areas (Figure 3.4.3B). 


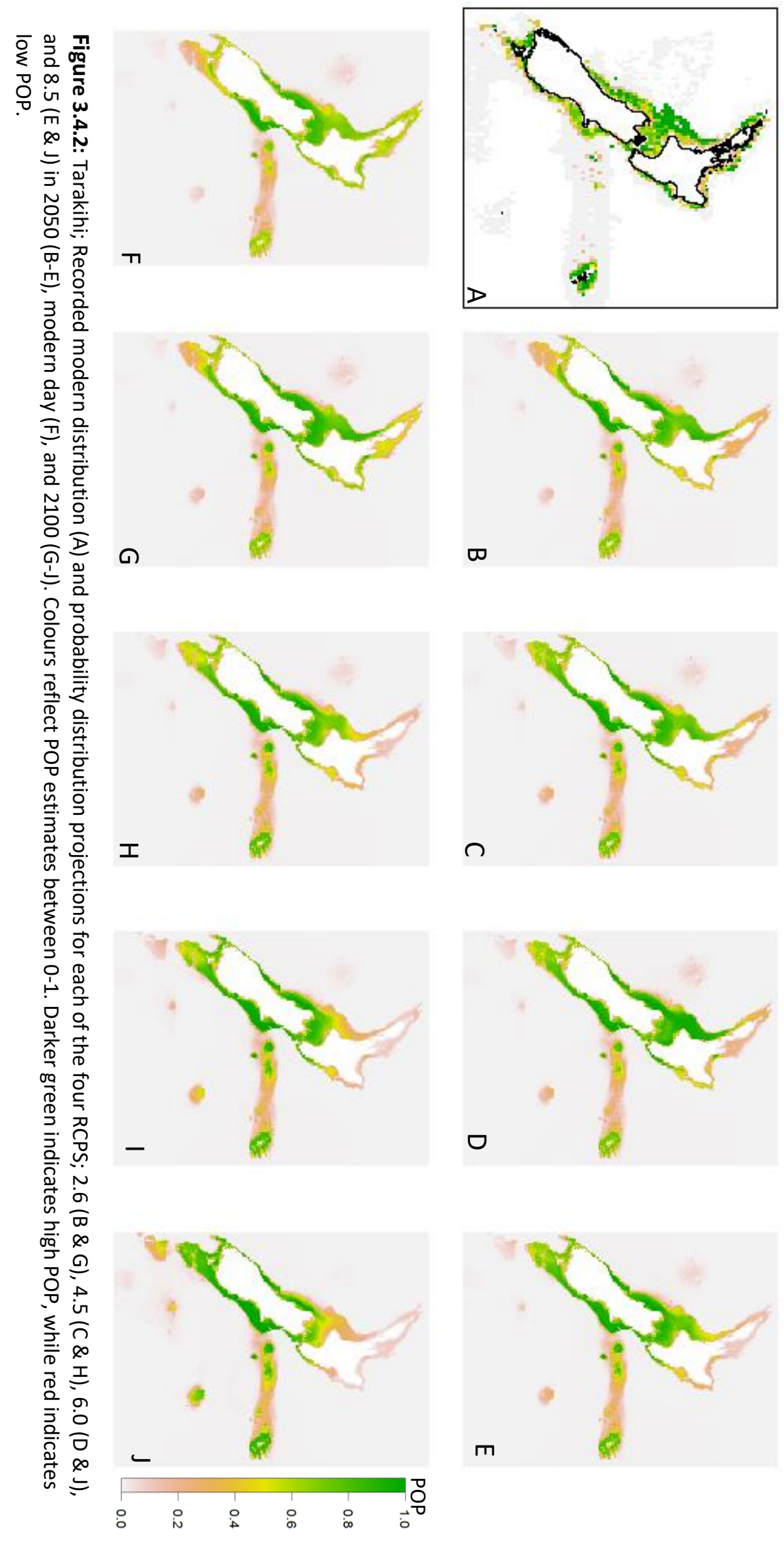


Table 3.4.2: Tarakihi; Change in POP values compared to the modern projection for each of the four climate scenarios 2050 and 2100.

\begin{tabular}{lllllllll}
\hline & \multicolumn{9}{c}{$\mathbf{2 0 5 0}$} & \multicolumn{4}{c}{$\mathbf{2 1 0 0}$} \\
POP & RCP2.6 & RCP4.5 & RCP6.0 & RCP8.5 & RCP2.6 & RCP4.5 & RCP6.0 & RCP8.5 \\
\hline $\mathbf{0 . 9}$ & 77.54 & 97.725 & 311.161 & 144.73 & 82.542 & 87.588 & 162.342 & 167.253 \\
$\mathbf{0 . 8}$ & 35.168 & 57.645 & 122.528 & 71.775 & 79.087 & 35.919 & 90.324 & 79.068 \\
$\mathbf{0 . 7}$ & 35.235 & 55.402 & 68.245 & 58.201 & 48.358 & 34.879 & 46.961 & 37.438 \\
$\mathbf{0 . 6}$ & -4.752 & 12.546 & 15.199 & 10.111 & 6.516 & -0.84 & -1.692 & -9.611 \\
$\mathbf{0 . 5}$ & -13.537 & -0.065 & 1.617 & -1.2 & 0.283 & -6.485 & -9.655 & -18.222 \\
$\mathbf{0 . 4}$ & -10.46 & -2.178 & -2.516 & -2.382 & 4.013 & -5.689 & -8.355 & -16.49 \\
$\mathbf{0 . 3}$ & -5.422 & -3.455 & -3.607 & -3.595 & 3.317 & -5.049 & -5.928 & -12.474 \\
$\mathbf{0 . 2}$ & -1.632 & -1.277 & -2.421 & -3.706 & 1.555 & -4.466 & -4.894 & -9.852 \\
$\mathbf{0 . 1}$ & -0.829 & 0.064 & -1.479 & -1.735 & 1.512 & -0.632 & -2.232 & -11.388 \\
\hline
\end{tabular}
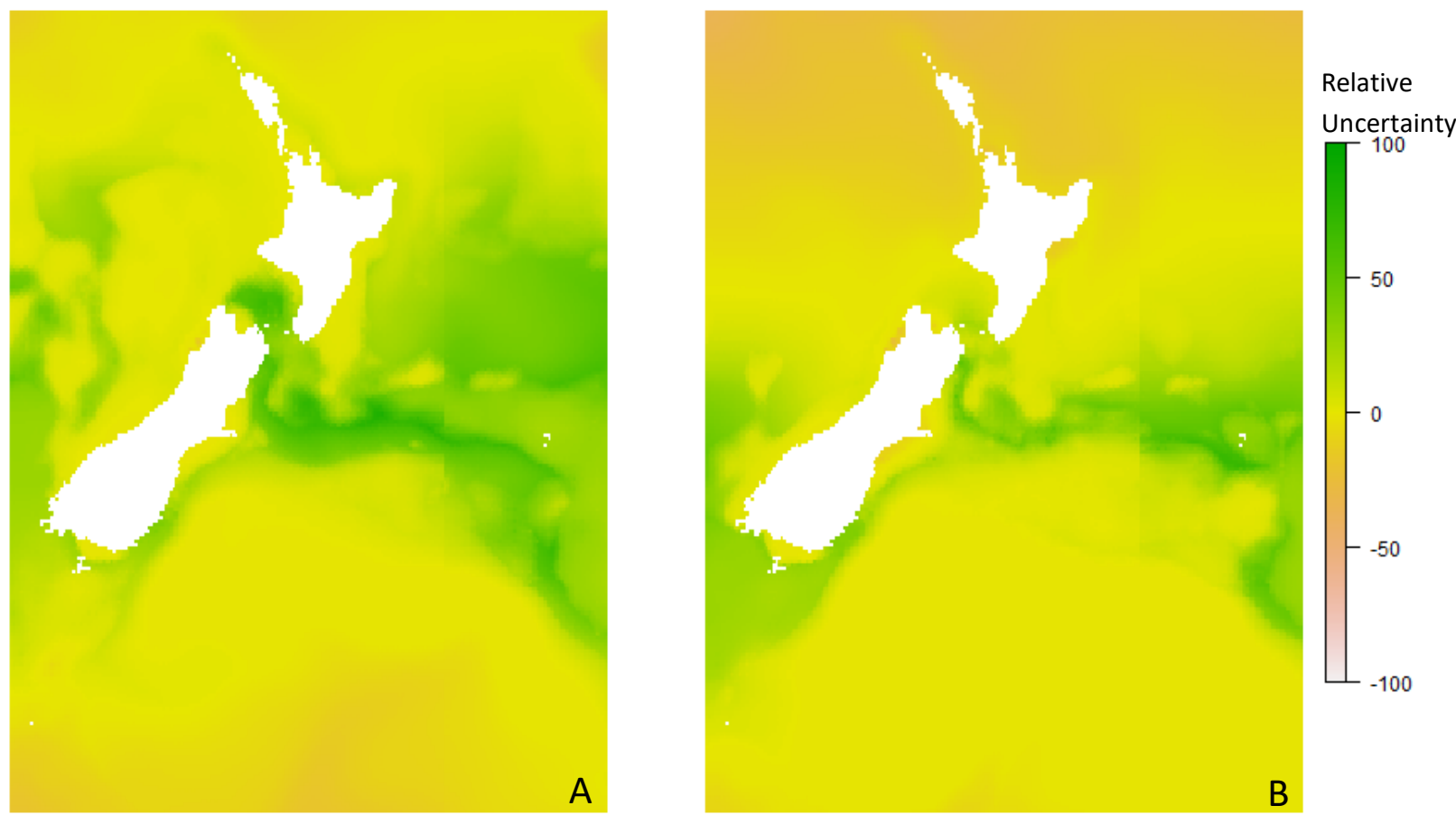

Figure 3.4.3: Tarakihi; MESS Maps of RCPS 2.62050 (A) and RCPS 8.52100 (B). MESS maps for all climate scenarios can be found in Appendix D.4. Negative sites indicate most relative uncertainty and positive sites indicate most relative certainty. 


\section{Variable Influence}

The base model predicted that tarakihi were completely absent at depths below -1000 metres, preferred SS mean between 34 - 34.5 psu but above that had a negative response, and peak POP at an ST mean of just below 18 degrees, which then decreased (Figure 3.4.4). There was a slight positive relationship with SS range and ST range. ST Mean had the highest permutation importance out of all climatic variables, while SS Mean did not quite have as much weight. SS and ST range had comparatively low permutation importance's (Figure 3.4.5).

The contemporary limiting factor plot (Figure 3.4.6A), showed that in the south-eastern areas of New Zealand from the bottom S/W corner of the SI to the top N/E corner of the SI, the climatic variable limiting tarakihi distribution was ST Mean. This was reduced in the RCPS 8.5 limiting factor plot (Figure 3.4.6B) so that ST Mean was the most limiting variable only in small pockets of ocean in the future limiting factors plot. SS Mean remained the most limiting climate variable around the NI in both modern and future scenarios.
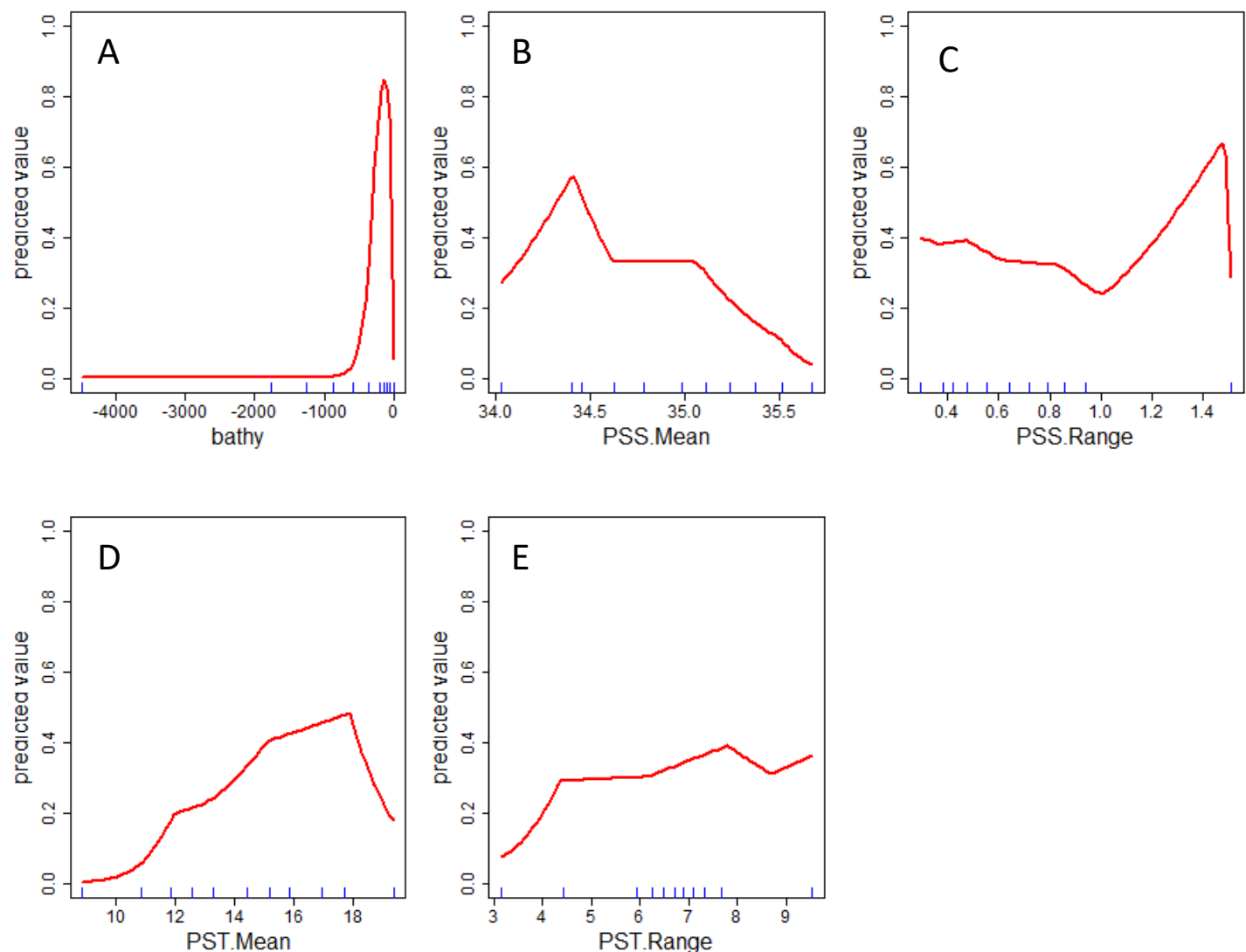

Figure 3.4.4: Tarakihi; Predictor response curves indicating how variables used for training affected the base model. Model predictions (red lines) and observations (blue data rug of deciles) are shown. The variables are bathymetry $(A)$, surface salinity mean $(B)$, surface salinity range $(C)$, surface temperature mean $(D)$, and surface temperature range $(E)$. 


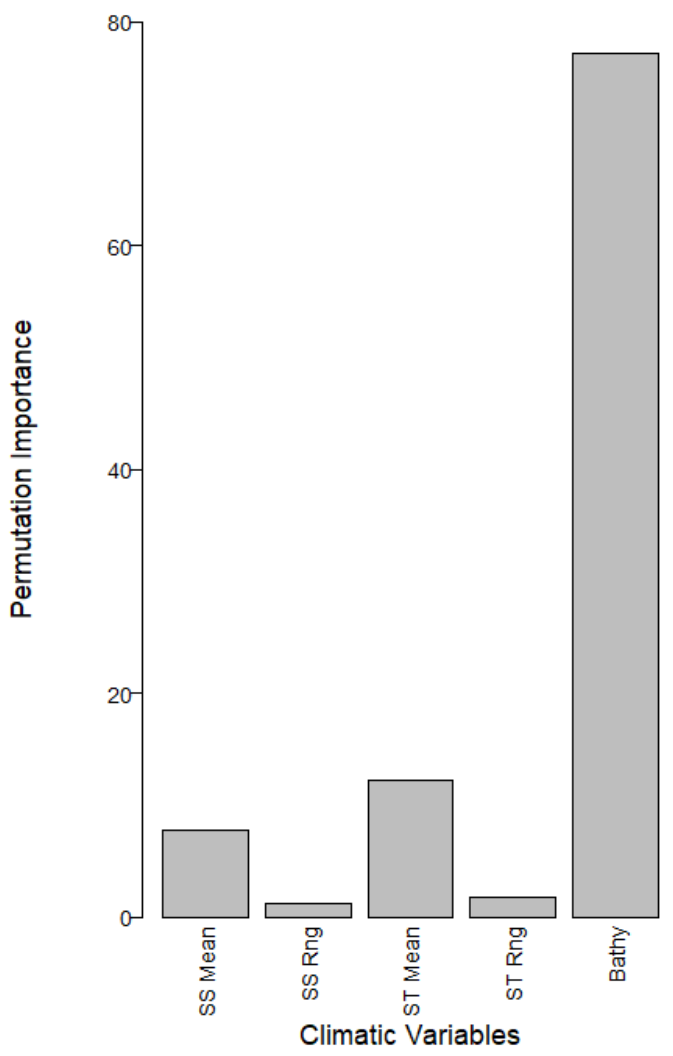

Figure 3.4.5: Tarakihi; Predictor variable contribution based on permutation importance (Phillips, 2006).

Variable names are shortened here to fit: SS = Surface salinity, ST = Surface temperature, Rng = range, Bathy = bathymetry.
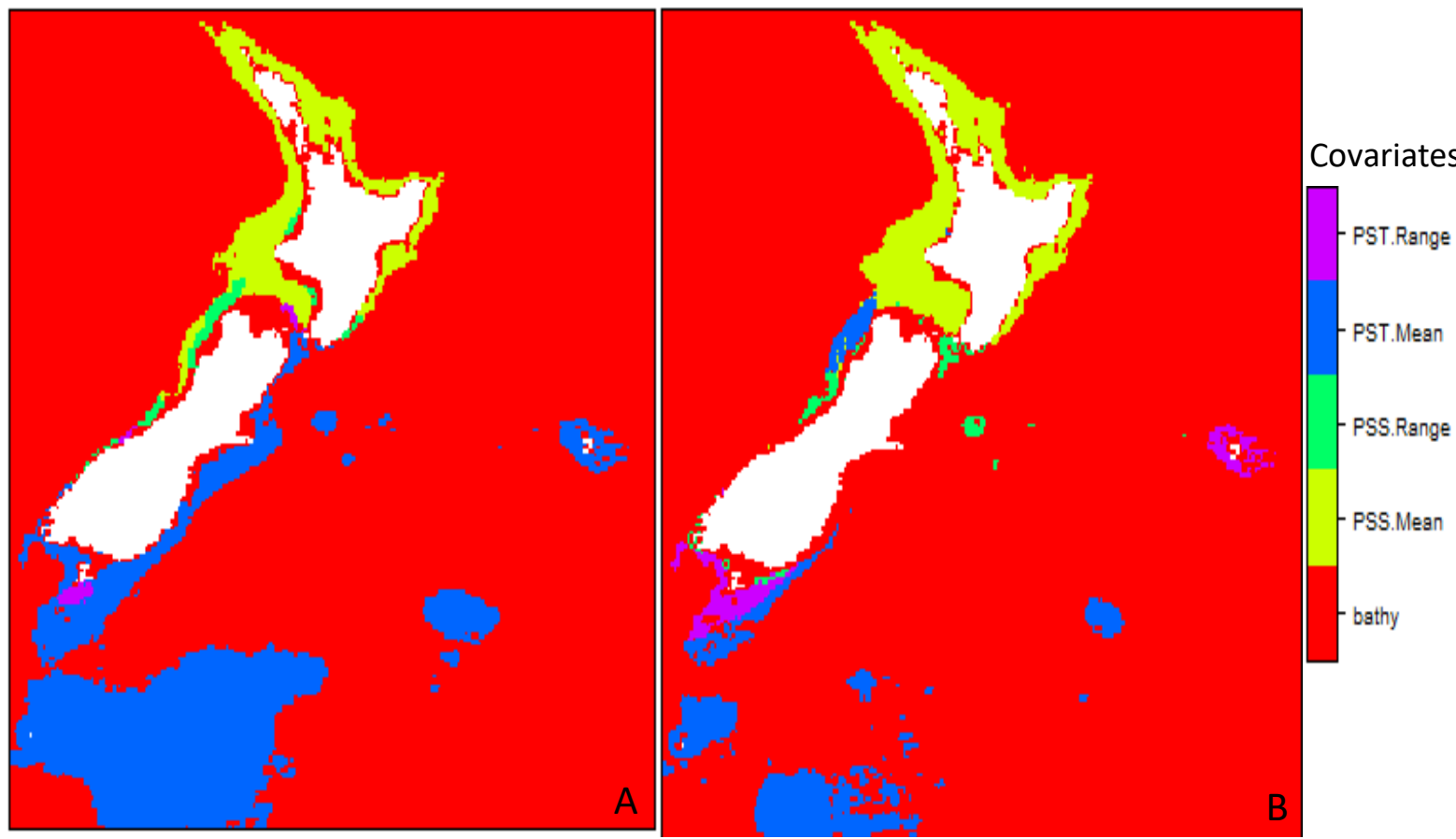

Figure 3.4.6: Tarakihi; Limiting factors plots of Modern (A), and RCPS 8.5 in 2100 (A) climates. Colours indicate the most limiting variable to distribution in that area. 


\section{5: SCAMPI (Metanephrops challenger)}

\section{$\underline{\text { Model Selection }}$}

Models selected are shown in table 3.5.1. $\triangle$ AIC values varied form 0 - 5764.19. Only models with a $\triangle \mathrm{AIC}$ of 0 were selected. Train AUC scores varied from $0.55-0.98$ and test AUC from $0.59-0.98$. To select models with good discriminatory ability only those $>0.75$ were selected. To select models with low overfitting average test $\mathrm{OR}_{10}$ values varied from $0-0.22$ but only those that were $<0.13$ were selected and average $A \cup C_{\text {DIFF }}$ varied from $0-0.11$ but only those $<0.05$ were selected. When selected models that fit these criteria were projected onto modern climate, projections were all very similar (Figure 3.5.1). All projections fit the known contemporary scampi distribution relatively well, although under predicted in the southernmost areas. Although models 2a10 and 4a11 had highest discriminatory ability and the lowest overfitting metrics $\left(\mathrm{OR}_{10}=0.103 \& 0.111, \mathrm{AUC}_{\mathrm{DIFF}}=0.004 \&\right.$ 0.003 respectively), no climatic variables contributed to either model fit (Table 3.5.1). Therefore the next best model that did show an environmental response was selected, which was only slightly more over. 1c4 was selected as the base model from the remaining models as it the lowest overfitting $\left(\mathrm{OR}_{10}=0.123, \mathrm{AUC}_{\mathrm{DIFF}}=0.017\right)$ and highest discriminatory ability $\left(\mathrm{AUC}_{\text {TRAIN }}=0.893, \mathrm{AUC}_{\text {TEST }}\right.$ $=0.869)$ of these. 


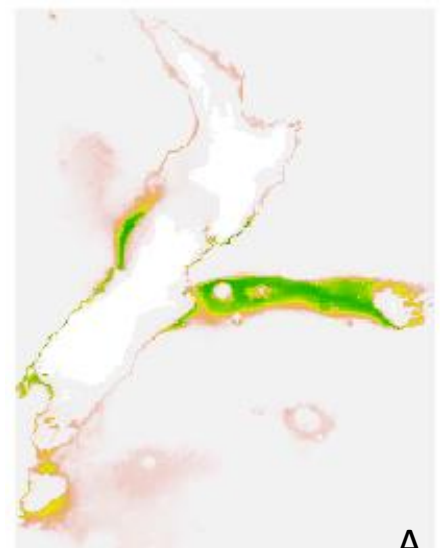

A

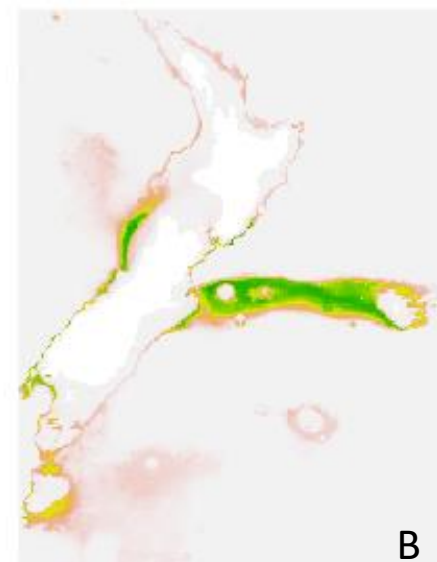

B
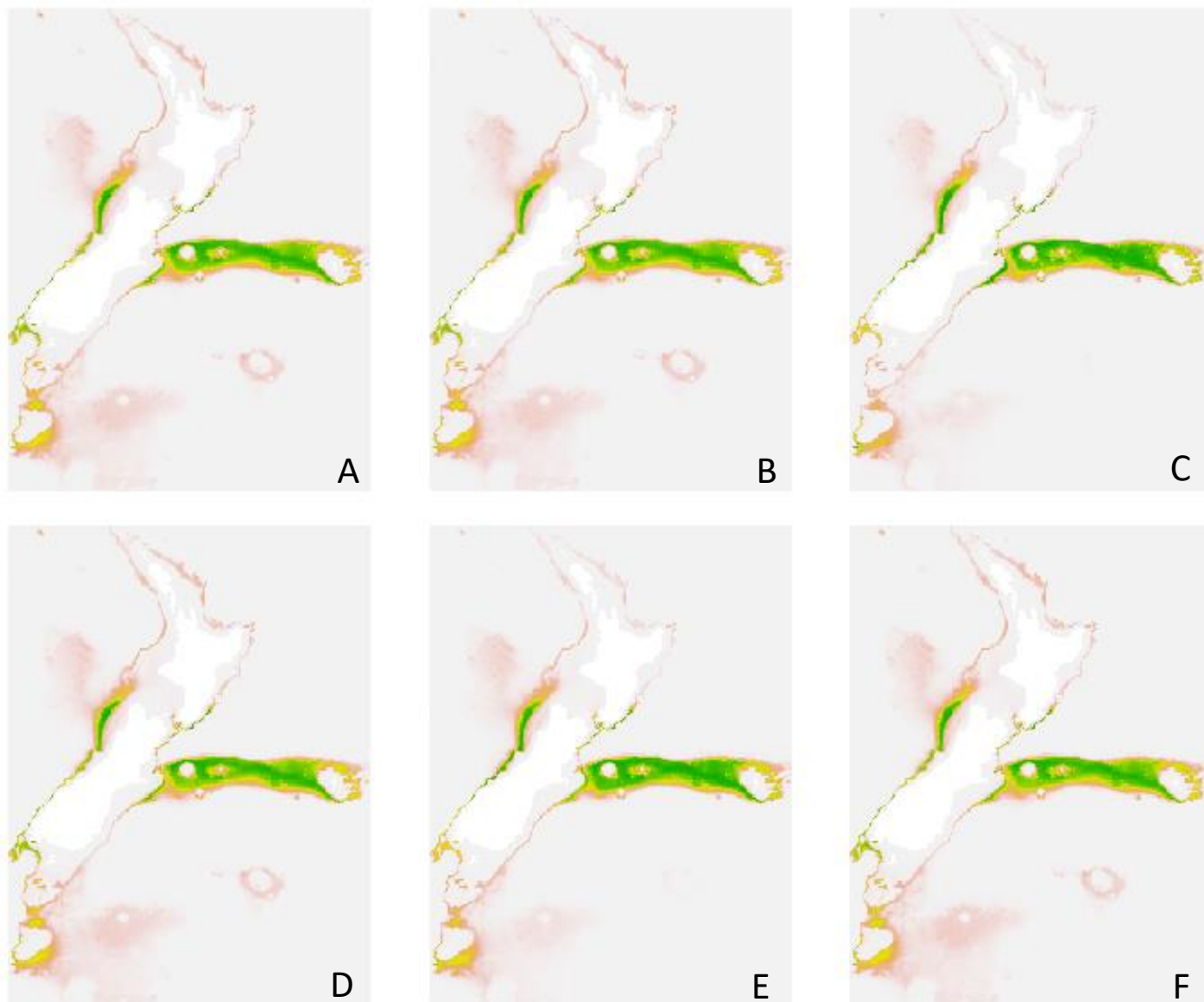

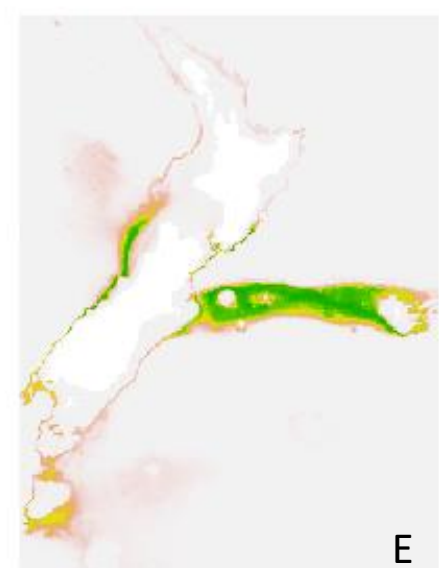

E

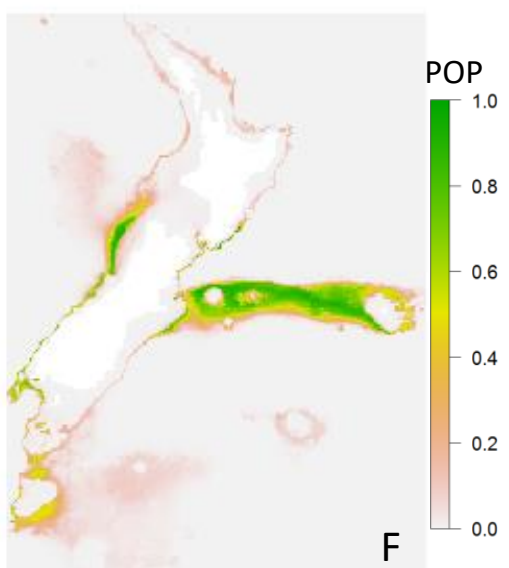

F

Figure 3.5.1: Scampi; Six best Maxent models projected on modern climate layers; 1c3 (A), 1c4 (B), 2a10 (C), 2c3 (D), 4a11 (E), 5c3 (F). Colours reflect probability of presence estimates between $0-1$. Darker green indicates higher POP and red the reverse. 


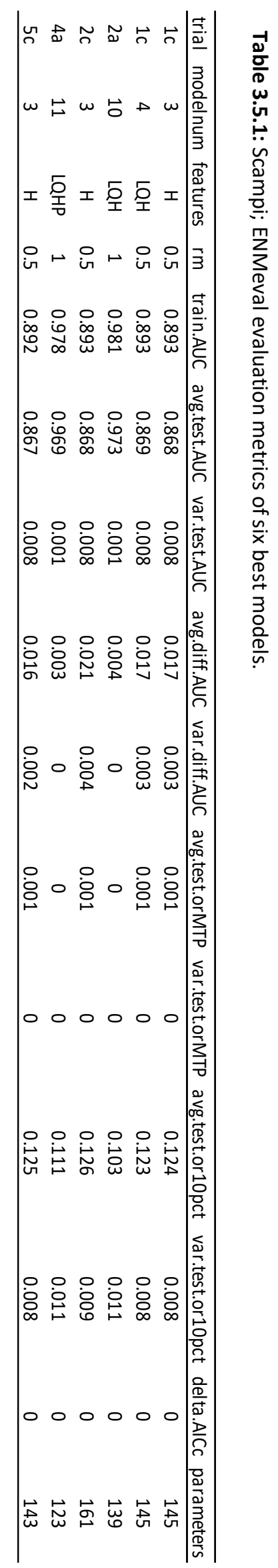




\section{Model Projections}

The 2050 predictions for each RCPS all looked relatively similar (Figure 3.5.2B-E). Main differences between the 2050 projections and the modern projection included increased POP in the southernmost region of the study area, southward of the Auckland Islands, and the varied POP on the Chatham Rise in different climate scenarios. POP on the Chatham Rise appeared to fluctuate for each scenario. Areas with a POP of $>0.9$ increased much more than that in any other POP bin (Table 3.5.2). Areas with a POP of $>0.9$ increased by $207.91-594.65 \%$, while areas with a POP between 0.5 and 0.6 decreased in RCPS 2.6 but in the other scenarios increased by $4.28-18.64 \%$. The scenario with the largest increase of POP across all bins, was RCPS 6.0, with the exception of bins 0.2 and 0.1 .

The 2050 MESS maps all looked very similar for each RCPS (Figure 3.5.3A, Appendix D.5). Notable areas with the high relative uncertainty included coastal areas around the NW corner of the SI, the middle of the East Coast of the SI around Banks Peninsular, and Hawke Bay. Places with high relative certainty were around the Chatham Rise, Cook Strait, and most regions around the SI. Slightly more uncertainty was present in the RCPS 8.5 MESS map, particularly in northern regions around the top of the NI.

\section{$\underline{2100 \text { Model Projections }}$}

The 2100 projections were more varied, as some scenarios deviated more from the contemporary prediction (Figure 3.5.2G-J). POP in RCPS 8.5 and RCPS 4.5, substantially increased in southern regions and along the Chatham Rise. In RCPS 8.5, suitable conditions around the NW corner of the SI disappeared almost entirely. In RCPS 2.6, POP appeared to decrease on the eastern end of the Chatham Rise. Like in the 2050 predictions, highly preferable conditions increased more than less preferable conditions, particularly in RCPS 6.0 and 8.5. Areas with a POP of $>0.9$ increased much more than that assigned to any other POP bin (Table 3.5.2). Area with a POP of $>0.9$ increased by $107.34-1401.74 \%$, while areas with a POP of $>0.5$ increased by $4.33-71.58 \%$. The largest increase of POP across all bins was in RCPS 8.5. Consistently, areas with high POP values became more prevalent, while areas with average POP values increased less, stayed the same or decreased. Areas with lower POP values increased also, but not as much high POP values.

The 2100 MESS maps had more variation, with more relative uncertainty in general (Appendix D.5). These displayed the same notable areas with the high relative uncertainty around the NW corner of the SI, the middle of the East Coast of the SI around Banks Peninsular, and Hawke Bay. The around the top of the $\mathrm{NI}$ also had relatively high uncertainty. This was most pronounced in the RCPS 8.5 MESS map (Figure 3.5.3B). Most uncertainty throughout the study region was in RCPS 8.5. 


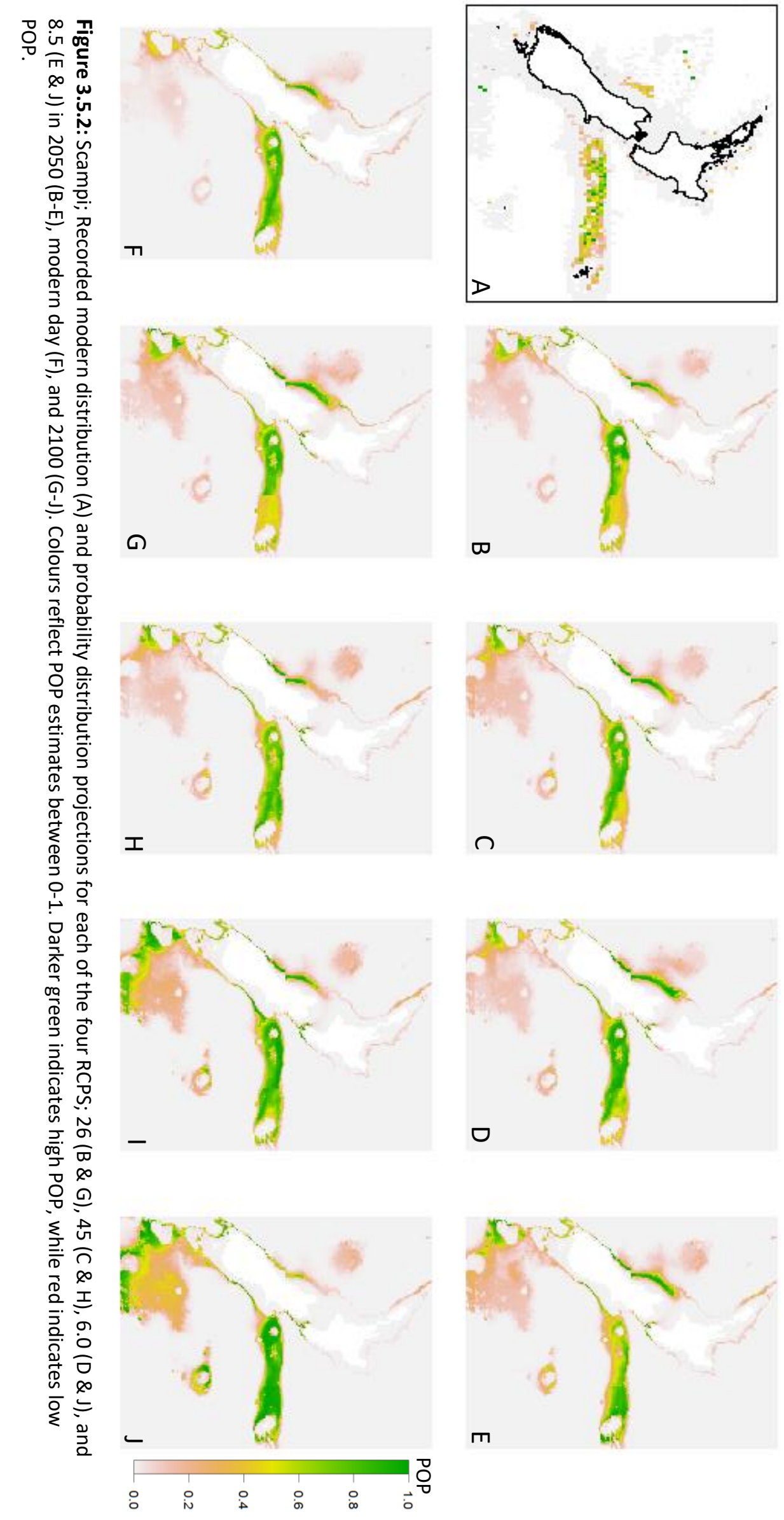


Table 3.5.2: Scampi; Change in POP values compared to the modern projection for each of the four climate scenarios 2050 and 2100 .

\begin{tabular}{lllllllll}
\hline \multicolumn{9}{c}{$\mathbf{2 0 5 0}$} \\
POP & RCP2.6 & RCP4.5 & RCP6.0 & RCP8.5 & RCP2.6 & RCP4.5 & RCP6.0 & RCP8.5 \\
\hline $\mathbf{0 . 9}$ & 207.908 & 194.34 & 594.645 & 247.194 & 107.335 & 40.679 & 656.234 & 1401.74 \\
$\mathbf{0 . 8}$ & 0.018 & 33.58 & 80.577 & 13.491 & 2.488 & -16.679 & 88.854 & 202.607 \\
$\mathbf{0 . 7}$ & -11.824 & 14.04 & 28.574 & -9.988 & -8.789 & 2.072 & 72.014 & 104.782 \\
$\mathbf{0 . 6}$ & -10.069 & 5.152 & 17.853 & -3.549 & -3.138 & 7.292 & 60.589 & 74.544 \\
$\mathbf{0 . 5}$ & -6.585 & 5.92 & 18.643 & 4.276 & 4.325 & 5.861 & 59.916 & 71.576 \\
$\mathbf{0 . 4}$ & 3.609 & 7.085 & 14.014 & 6.417 & 13.666 & -1.231 & 50.09 & 77.898 \\
$\mathbf{0 . 3}$ & 5.084 & 7.313 & 11.45 & 12.746 & 9.575 & 0.147 & 46.865 & 102.211 \\
$\mathbf{0 . 2}$ & 6.903 & 13.97 & 11.727 & 32.31 & 12.406 & 5.199 & 69.744 & 103.934 \\
$\mathbf{0 . 1}$ & 27.936 & 53.742 & 43.459 & 69.62 & 45.853 & 41.283 & 81.94 & 93.591 \\
\hline
\end{tabular}
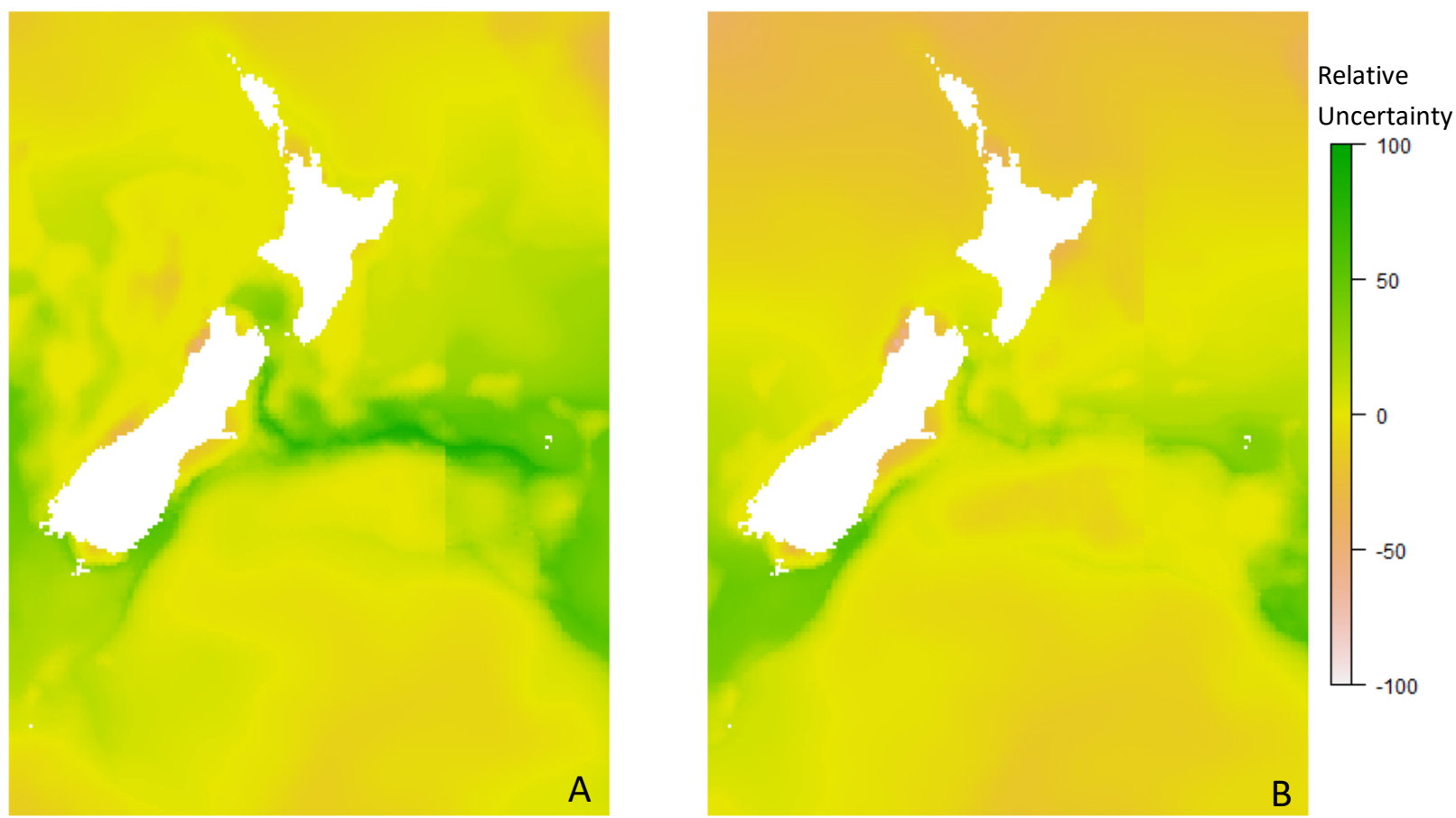

Figure 3.5.3: Scampi; MESS Maps of RCPS 2.62050 (A) and RCPS 8.52100 (B). MESS maps for all climate scenarios can be found in Appendix D.5. Negative sites indicate most relative uncertainty and positive sites indicate most relative certainty. 


\section{Variable Influence}

Response curves indicated scampi were completely absent at depths below -1000 metres, had decreased POP with SS Mean, and increased POP with ST Mean (Figure 3.5.4). Although there was a clear increase in POP with mean ST, which peaked around 16 degrees, information beyond 18 degrees was outside of the range of the data so a constant value was assumed and POP was fixed at about 18 degrees at about 0.75 . Responses of SS and ST range and SS mean were relatively cryptic, and did not seem plausible, but SS and ST range contributed less to the base model than other variables (Figure 3.5.4 \& Figure 3.5.5). ST mean had a substantially higher permutation importance than other climatic variables (Figure 3.5.5).

The contemporary limiting factor plot (Figure 3.5.6A), showed ST mean was the main climatic limiting factor from the lower Chatham Rise southward, including along the eastern side of the SI to the S/W corner of the SI. In the future limiting plot, this effect of ST mean was mainly limited to on and around the Campbell plateau (Figure 3.5.6B). The main limiting factor in northern New Zealand from the mid SI northwards in both contemporary and future limiting plots was SS Mean. In the future limiting plot ST range became a main limiting factor around the lower Chatham rise and S/W corner of the Sl.
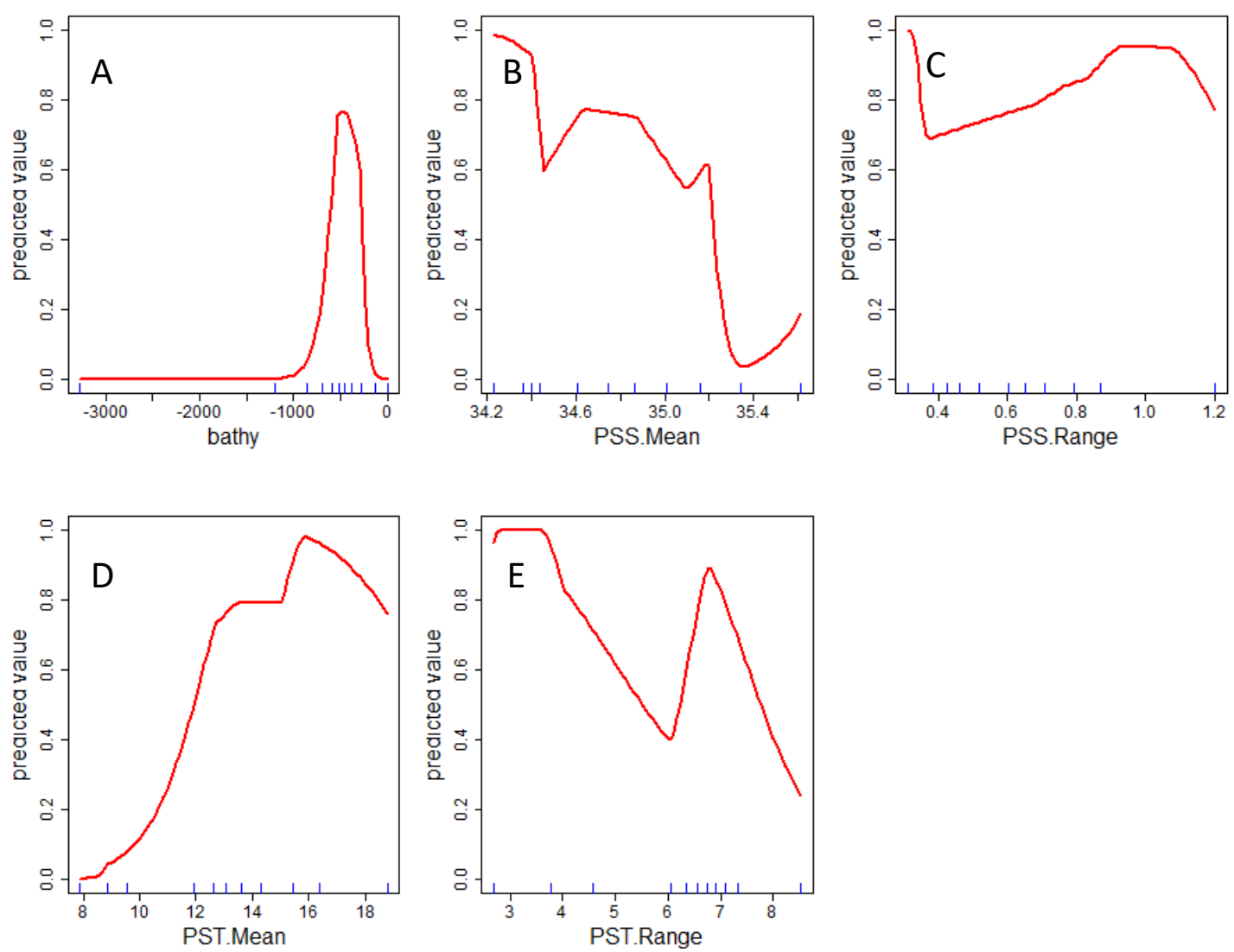

Figure 3.5.4: Scampi; Predictor response curves indicating how variables used for training affected the base model. Model predictions (red lines) and observations (blue data rug of deciles) are shown. The variables are bathymetry $(A)$, surface salinity mean $(B)$, surface salinity range $(C)$, surface temperature mean $(D)$, and surface temperature range $(E)$. 


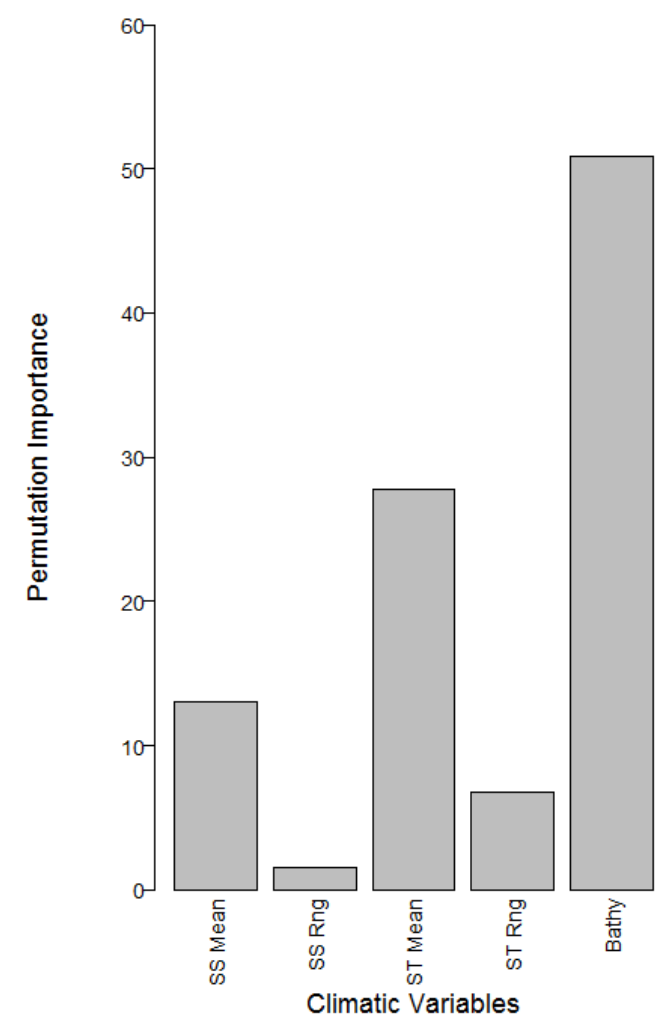

Figure 3.5.5: Scampi; Predictor variable contribution based on permutation importance (Phillips, 2006). Variable names are shortened here to fit: SS = Surface salinity, ST = Surface temperature, Rng = range, Bathy = bathymetry.

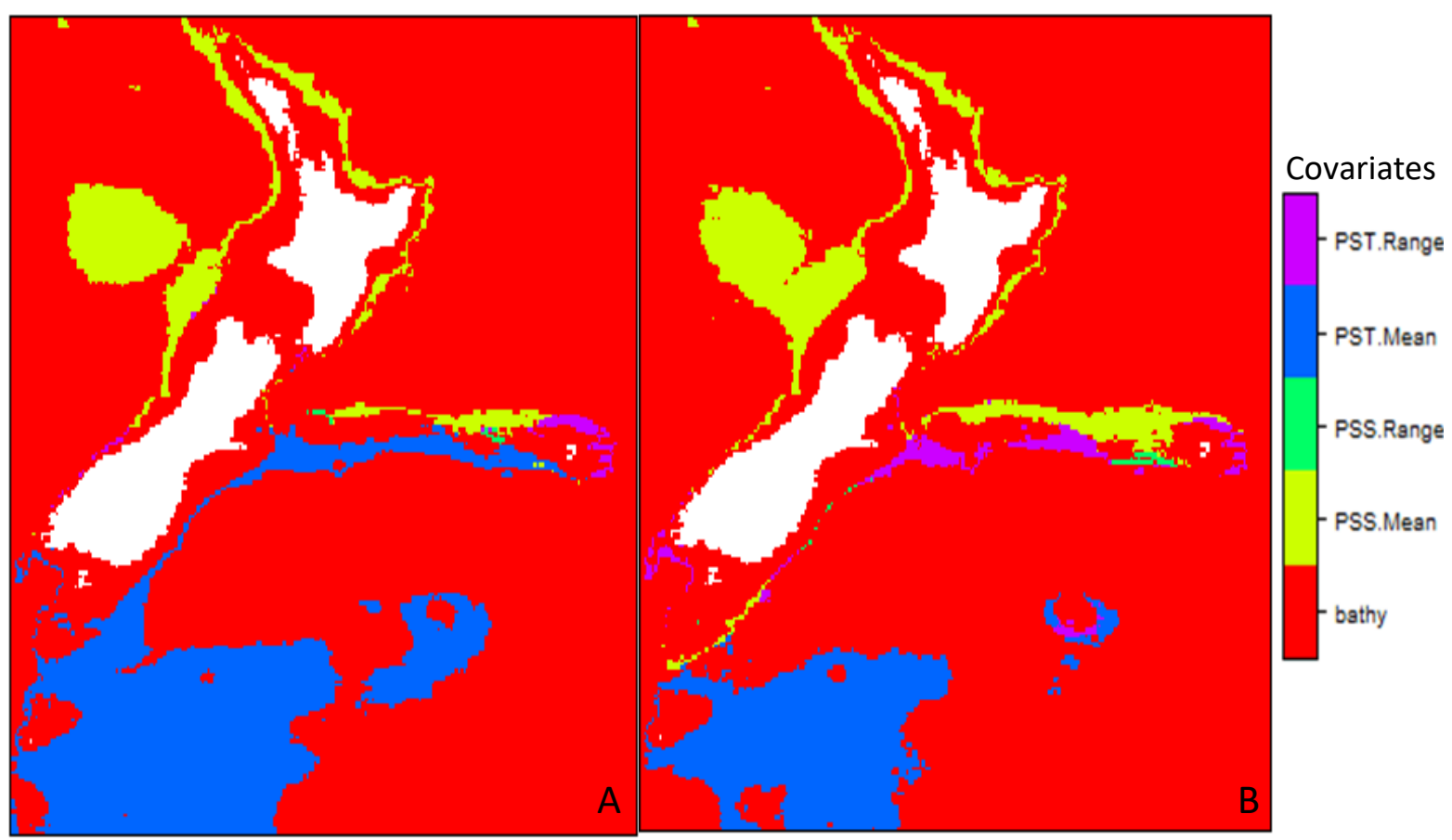

Figure 3.5.6: Scampi; Limiting factors plots of Modern (A), and RCPS 8.5 in 2100 (A) climates. Colours indicate the most limiting variable to distribution in that area. 


\section{6: ORANGE ROUGHY (Hoplostethus atlanticus)}

\section{$\underline{\text { Model Selection }}$}

$\triangle A I C$ values varied form 0 - 97463.59. Only models with a $\triangle A I C$ of 0 were selected. Train AUC scores varied from $0.56-0.98$ and test AUC from 0.66 - 0.97. To select models with good discriminatory ability only those $>0.75$ were selected. To select models with low overfitting average test $\mathrm{OR}_{10}$ values varied from 0 - 0.264 but only those that were $<0.11$ were selected, average $A \cup C_{\text {DIFF }}$ varied from 0 0.16 , but only those $<0.05$ were selected. The models that fit these criteria are shown in Table 3.6.1. When projected onto the contemporary climate the probability distributions for each model were greatly varied (Figure 3.6.1). Probability distributions of models $3 d 5,3 d 6$ and $4 d 4$ (Figure

3.6.1B, C\&D), were more conservative than the other models, while models $2 \mathrm{e} 2,4 \mathrm{e} 2$ and $5 \mathrm{e} 2$ (Figure 3.6.1A,D\&E) seemed to overestimate Roughy modern distribution. The more conservative models, $3 d 5,3 d 6$ and $4 d 4$ all had higher discriminatory ability $\left(A \cup C_{\text {TRAIN }}=0.934-0.947, A U C_{\text {TEST }}=0.921-\right.$ 0.929) than the overestimated models, but also all had had response curves that contrasted known ecology of orange roughy so these three models were ruled out as choices for the base model. Of the remaining models, $2 \mathrm{e} 2$ had the lowest average $\mathrm{OR}_{10}$ value $(0.08)$ than all other models, higher discriminatory ability $\left(A \cup C_{\text {TRAIN }}=0.816, A \cup C_{\text {TEST }}=0.819\right)$, and appeared to overestimate distribution the least. Expert opinion subsequently suggested 2e2 described the contemporary distribution of orange roughy better than the other models (M Dunn, NIWI, pers. comm). Model 2e2 was therefore selected as the base model for orange roughy projections. 

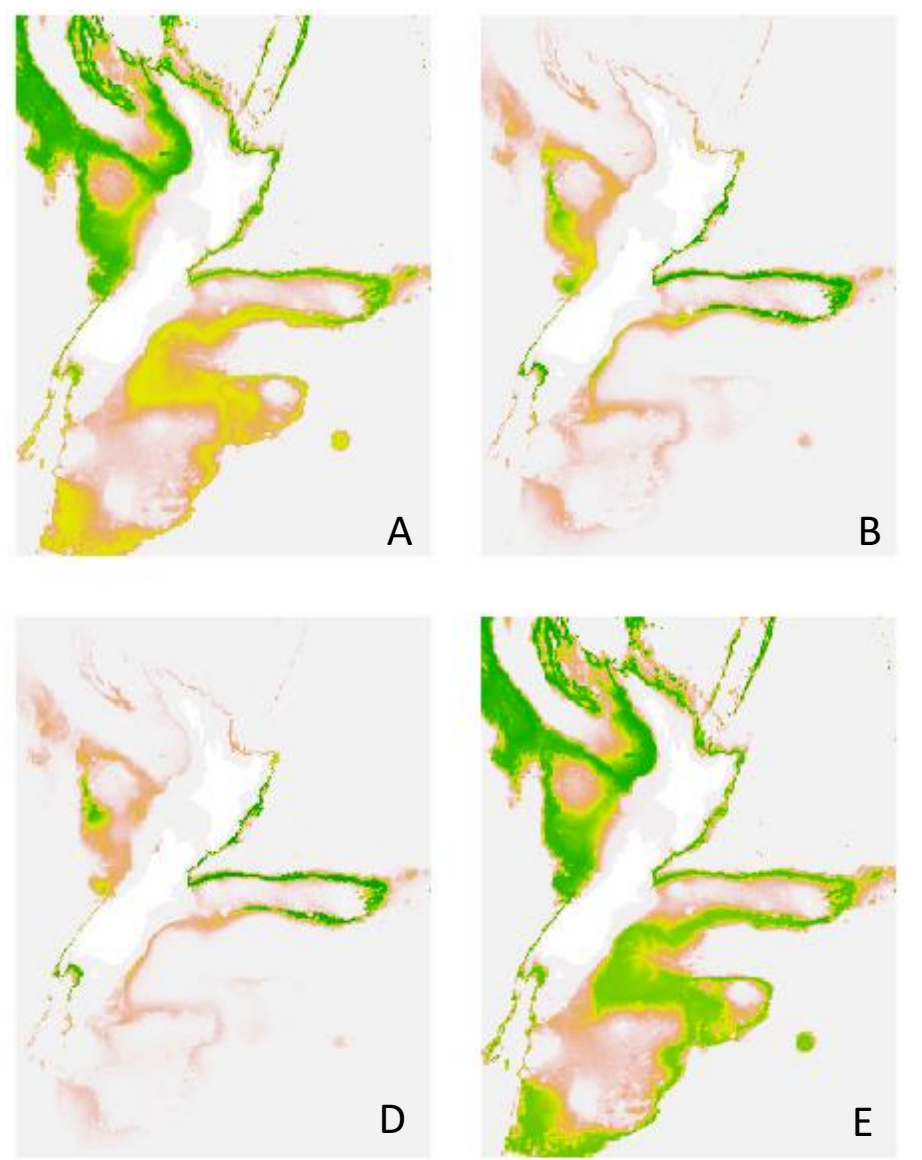
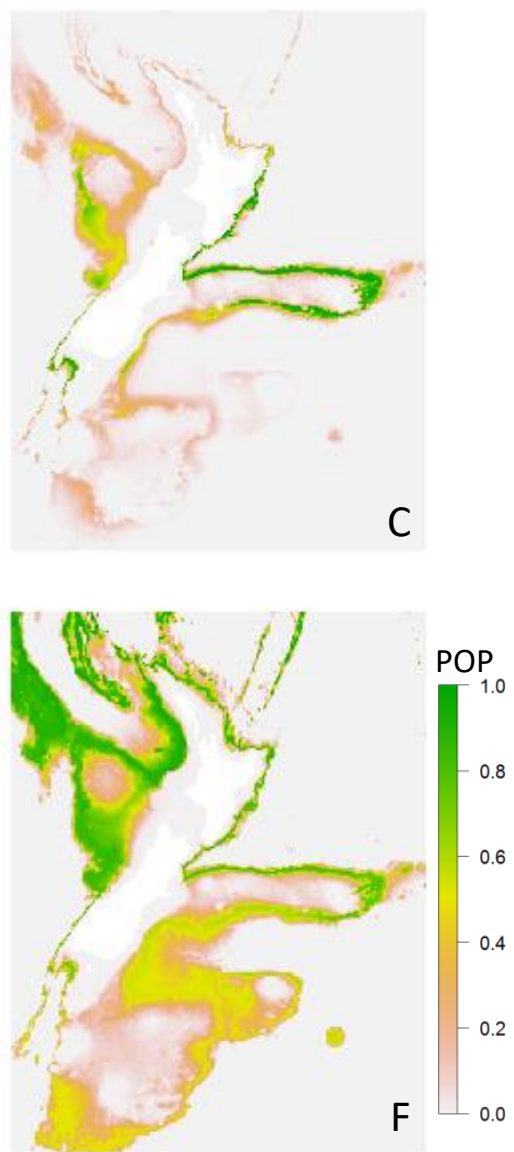

Figure 3.6.1: Orange roughy; Six best Maxent models projected on modern climate layers; $2 e 2$ (A), $3 d 5$ (B), 3d6 (C), $4 \mathrm{~d} 4(\mathrm{D}), 4 \mathrm{e} 2(\mathrm{E}), 5 \mathrm{e} 2(\mathrm{~F})$. Colours reflect probability of presence estimates between $0-1$. Darker green indicates higher POP and red the reverse. 


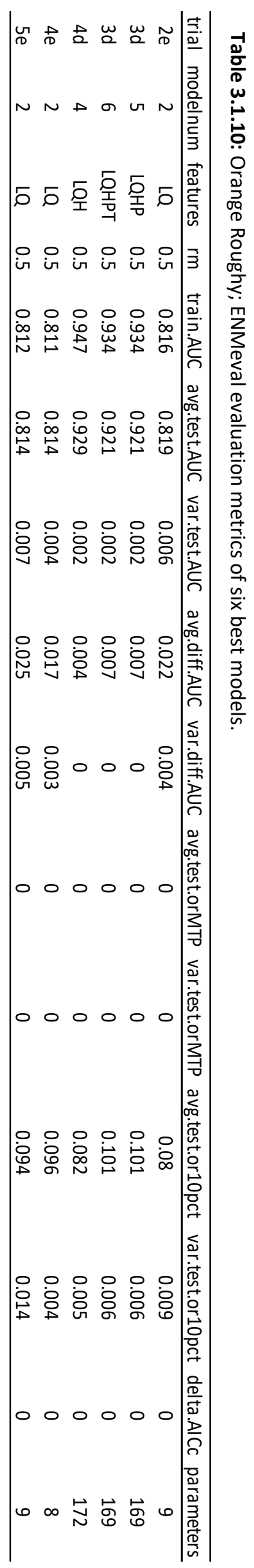




\section{Model Projections}

For the 2050 projections (Figure 3.6.2), all of the future climate scenarios appeared to showed similar results with southern areas gaining slightly higher POP scores in all scenarios. High POP of $>0$. 7 all around the NI, Lorde Howe Rise, Challenger Plateau, and halfway down the West Coast of the SI remained constant in all scenarios. The north of the Chatham rise also maintained a stable POP, while POP around the Southern Chatham Rise increased in all scenarios. Overall POP increased throughout the range of Roughy in all scenarios. The most extreme increases of POP were observed where POP was already high. In all scenarios, areas with a POP of $>0.9$ increased by more than that of any other lower POP bin (Table 3.6.2). Across all 2050 scenarios POP $>0.9$ increased by 38.2 $101.72 \%$ while areas with a POP between 0.5 and 0.6 increased by $17.09-27.49 \%$.

The 2050 MESS maps (Figure 3.6.2A) for all of these scenarios showed little relative uncertainty. Relative certainty was highest in the RCPS 2.6 prediction (Figure 3.6.3A) particularly around the Cook Strait, Chatham rise, and the East Coast of the $\mathrm{SI}$, indicating these were areas where the predicted 2050 conditions most closely represented contemporary climate in the base model. Areas with the most relative uncertainty were the most southern and northern regions at the very edges of the study area.

\section{$\underline{2100 \text { Model Projections }}$}

There was slightly more variation in the 2100 predictions (Figure 3.6.3). Not only did POP increase in general in all scenarios but similarly to the 2050 predictions, there was a larger increase in areas with a POP of $>0.9$ than that assigned to any POP bin (Table 3.6.2). Across all scenarios in 2100 POP $>0.9$ increased by $50-167.9 \%$ while POP between 0.5 - 0.6 increased by $16.2-50.8 \%$. RCPS 8.5 scenario clearly had the most pronounced increase in POP, while RCPS 6.0 had the second biggest increase. The most obvious differences were around the northern NI regions which had higher POP illustrated by the darker green areas, and the area around the Campbell Plateau and S/E of the NI, which was predicted to have an increase of POP from between $0.4-0.5$ to between $0.6-0.7$ in some areas.

The 2100 MESS map showed more uncertainty than the 2050 ones (Appendix D.6). RCPS 8.5 had substantially more uncertainty than all other RCPS, particularly northwards of the central NI, including Hawke Bay (Figure 3.6.3B). Another notable area of uncertainty in the 2100 MESS maps was just south-east of the Campbell Plateau. 

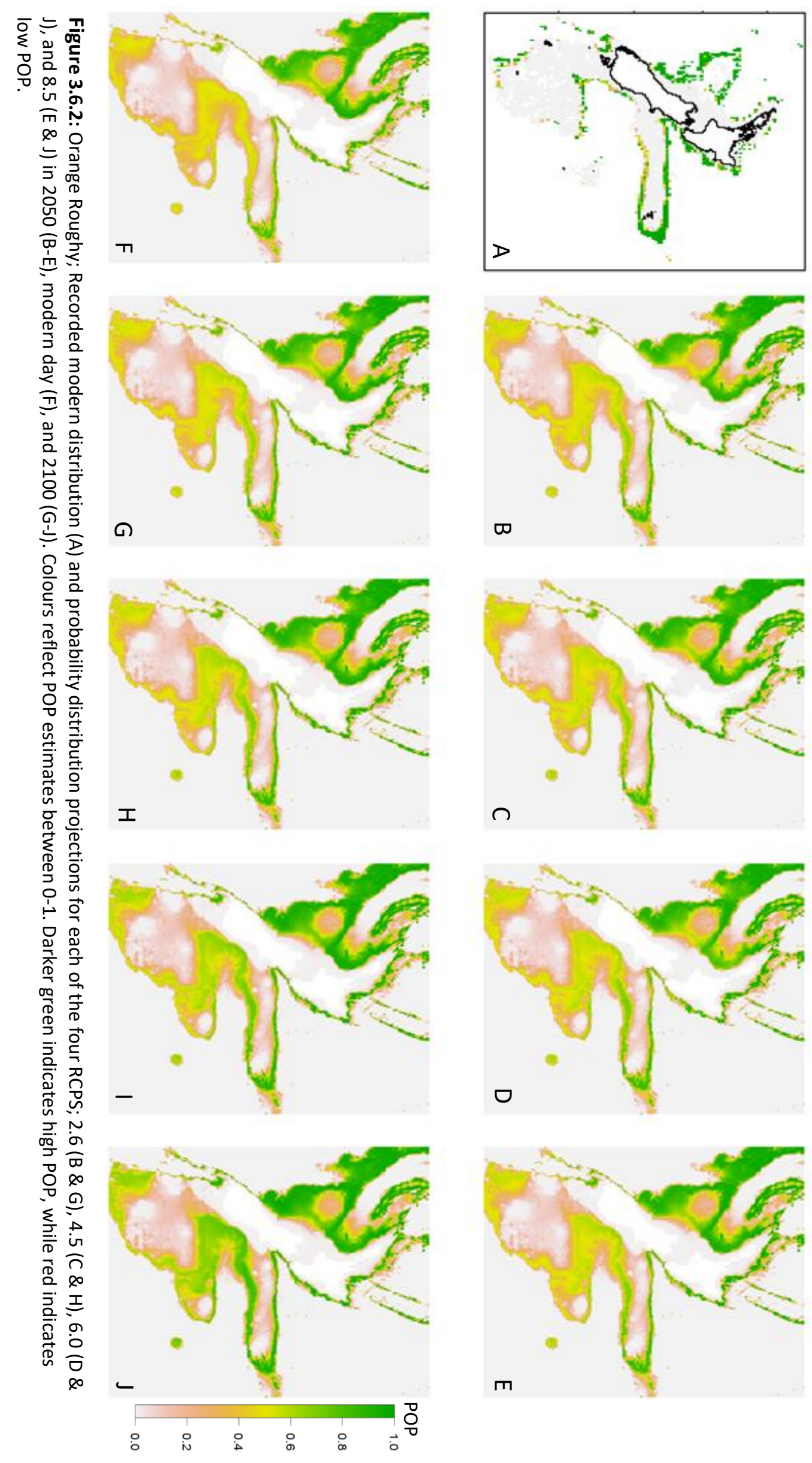
Table 3.6.2: Orange roughy; Change in POP values compared to the modern projection for each of the four climate scenarios 2050 and 2100.

\begin{tabular}{llllllllll}
\hline \multicolumn{7}{c}{$\mathbf{2 0 5 0}$} & \multicolumn{5}{c}{$\mathbf{2 1 0 0}$} \\
POP & RCP2.6 & RCP4.5 & RCP6.0 & RCP8.5 & RCP2.6 & RCP4.5 & RCP6.0 & RCP8.5 \\
\hline $\mathbf{0 . 9}$ & 38.202 & 63.016 & 40.999 & 101.721 & 49.99 & 76.093 & 145.279 & 167.943 \\
$\mathbf{0 . 8}$ & 6.082 & 16.057 & 2.619 & 20.786 & 8.693 & 19.238 & 34.15 & 45.501 \\
$\mathbf{0 . 7}$ & 2.211 & 8.004 & 3.535 & 11.121 & 5.586 & 9.798 & 23.487 & 41.655 \\
$\mathbf{0 . 6}$ & 4.803 & 11.518 & 8.778 & 13.784 & 9.062 & 17.301 & 35.766 & 66.011 \\
$\mathbf{0 . 5}$ & 17.085 & 26.11 & 25.406 & 27.492 & 16.15 & 29.244 & 41.381 & 50.83 \\
$\mathbf{0 . 4}$ & 4.341 & 7.374 & 6.694 & 8.014 & 5.066 & 8.252 & 12.991 & 17.601 \\
$\mathbf{0 . 3}$ & 2.565 & 4.539 & 4.236 & 5.028 & 3.086 & 5.099 & 8.09 & 11.167 \\
$\mathbf{0 . 2}$ & 1.876 & 2.979 & 2.898 & 3.288 & 2.142 & 3.589 & 5.696 & 8.272 \\
$\mathbf{0 . 1}$ & 1.951 & 2.614 & 2.844 & 2.665 & 1.643 & 2.936 & 4.949 & 7.126 \\
\hline
\end{tabular}
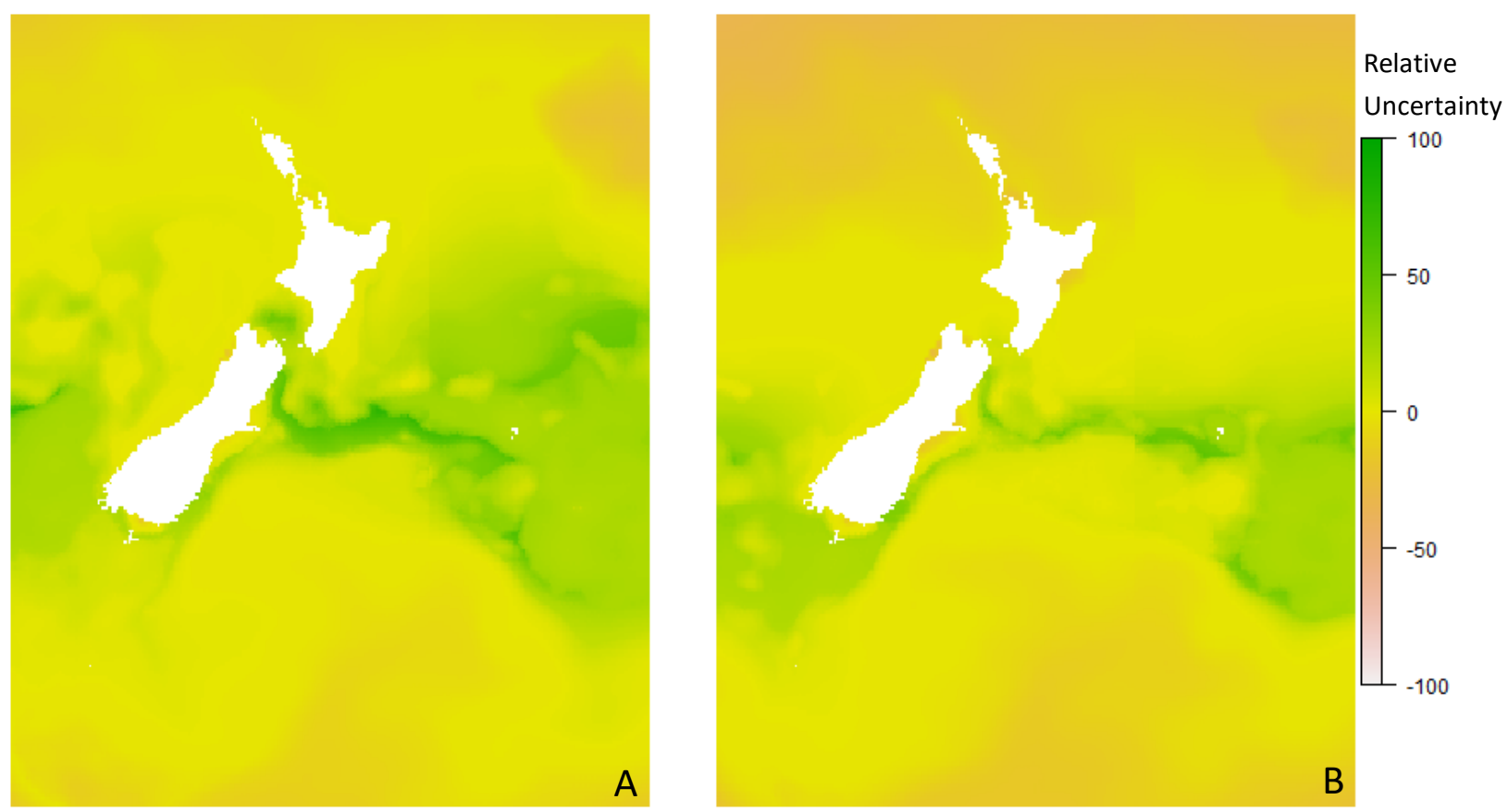

Figure 3.6.3: Orange roughy; MESS Maps of RCPS 2.62050 (A) and RCPS 8.52100 (B). MESS maps for all climate scenarios can be found in Appendix D.6. Negative sites indicate most relative uncertainty and positive sites indicate most relative certainty. 


\section{$\underline{\text { Variable Influence }}$}

The base model predicted that orange roughy were virtually absent at depths below $-2000 \mathrm{~m}$ and above $500 \mathrm{~m}$ and had increased POP with mean temperature (Figure 3.6.4). Information beyond 20 degrees was outside of the range of the data so a constant value was assumed and POP was fixed at 20 degrees at about 0.9 . The SS range curve suggested that a large range in salinity was slightly less preferable but permutation importance values (Figure 3.6.5) showed this was of little consequence. Temperature range and salinity mean curves predicted slight negative relationships with orange roughy POP but had little contribution to the base model (Figure 3.6.4 \& Figure 3.6.5).

The main climatic variable limiting contemporary distribution of orange roughy from the $\mathrm{S} / \mathrm{W}$ corner of the SI to the eastern edge of the Chatham rise was ST mean, while SS mean limited distribution most in $\mathrm{N} / \mathrm{W}$ regions, all around the $\mathrm{NI}$ and Challenger Plateau as well as the northern Chatham Rise (Figure 3.6.5). In the future limiting plot the area for which ST mean was a limiting factor was reduced, particularly around the Chatham Rise. Meanwhile, ST range, SS mean, and SS range primarily limited distribution on the Chatham rise. ST mean was still the main limiting around S/E New Zealand, and SS mean remained the main limiting factor around N/W New Zealand.
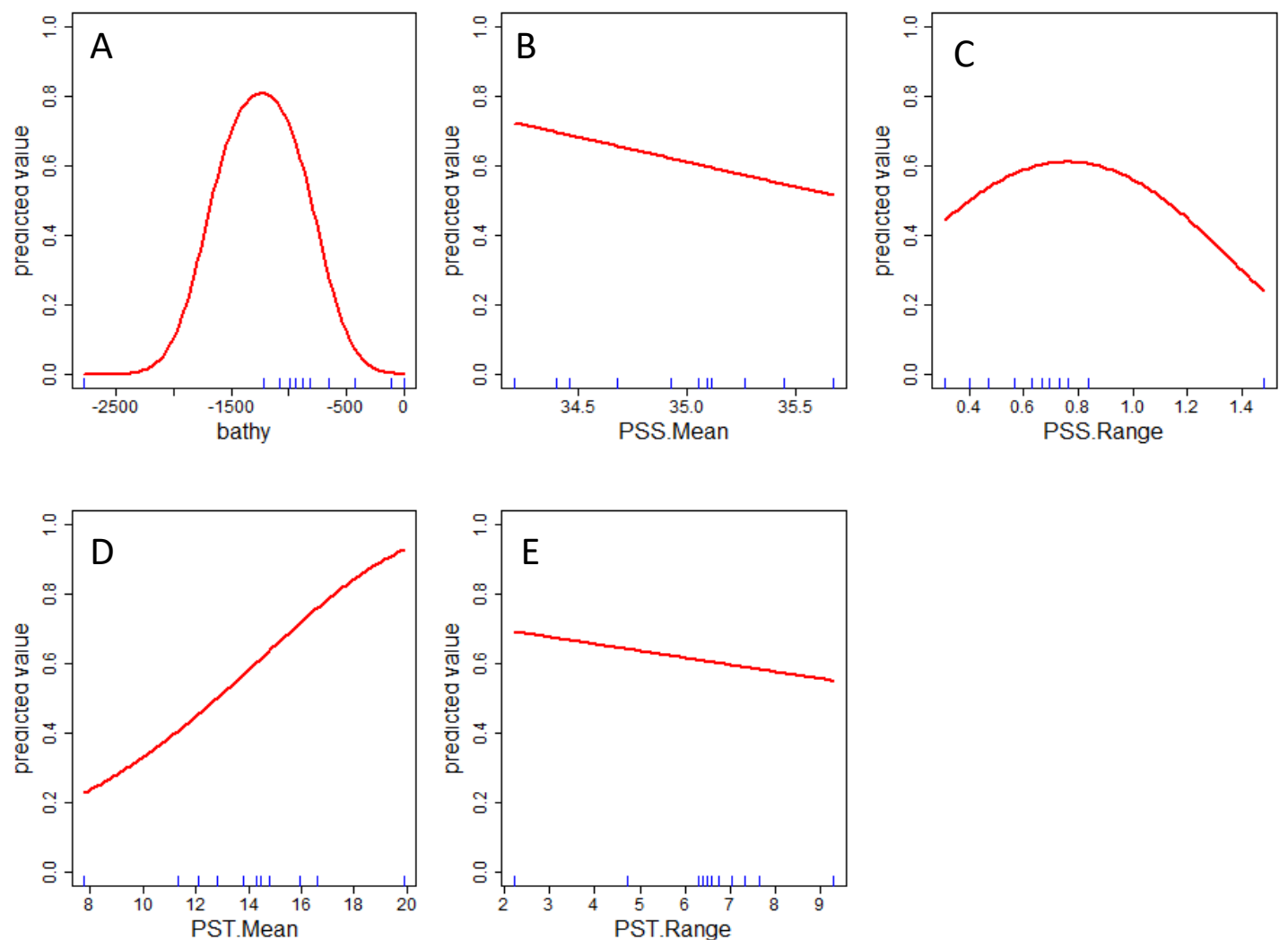

Figure 3.6.4: Orange roughy; Predictor response curves indicating how variables used for training affected the base model. Model predictions (red lines) and observations (blue data rug of deciles) are shown. The variables are bathymetry $(A)$, surface salinity mean $(B)$, surface salinity range $(C)$, surface temperature mean $(D)$, and surface temperature range $(E)$. 


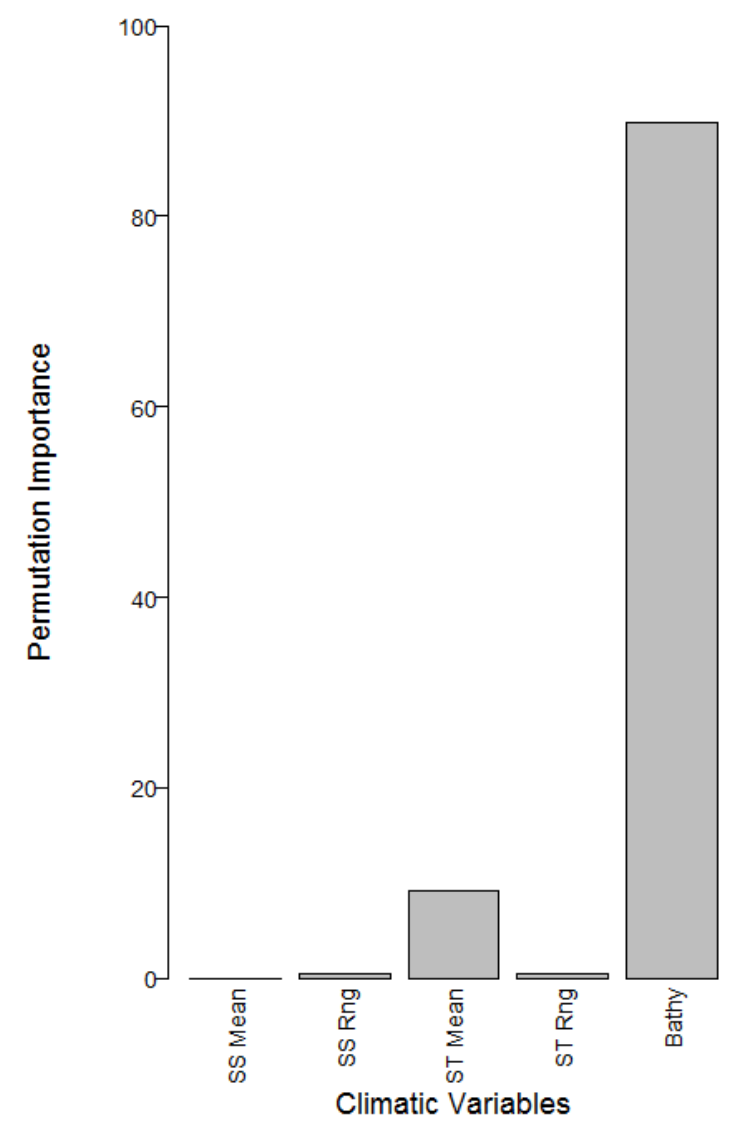

Figure 3.6.5: Orange roughy; Predictor variable contribution based on permutation importance (Phillips, 2006). Variable names are shortened here to fit: SS = Surface salinity, ST = Surface temperature, Rng = range, Bathy = bathymetry.
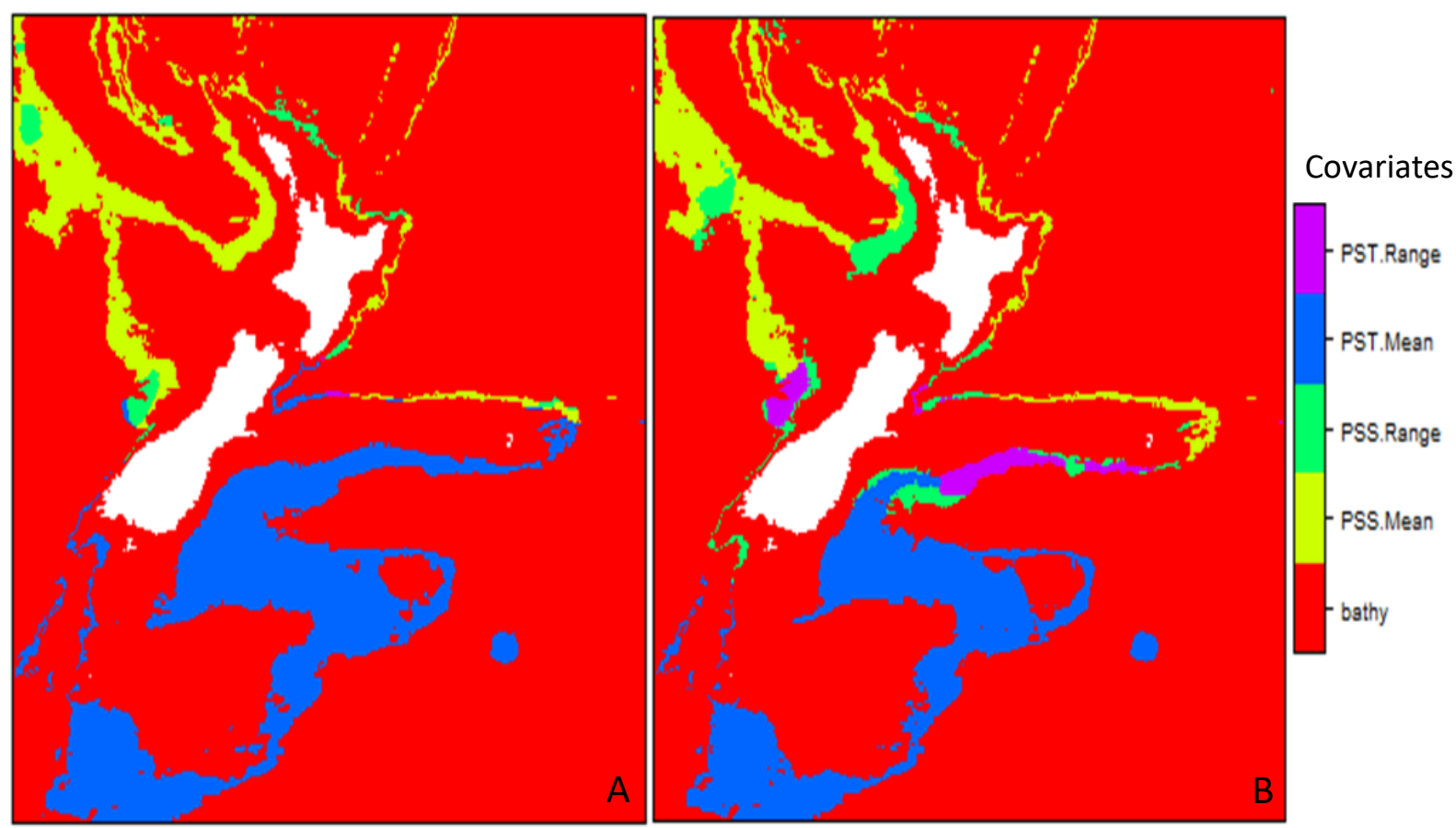

Figure 3.6.6: Orange roughy; Limiting factors plots of Modern (A), and RCPS 8.5 in 2100 (A) climates. Colours indicate the most limiting variable to distribution in that area. 


\subsection{SOUTHERN BLUE WHITING (Micromesistius australis)}

\section{$\underline{\text { Model Selection }}$}

Models selected are shown in table 3.1. $\triangle$ AIC values varied form 0 - 4387.3. Only models with a $\triangle A I C$ of 0 were selected. Train AUC scores varied from $0.54-0.98$ and test AUC from $0.55-0.98$. To select models with good discriminatory ability only those $>0.75$ were selected. To select models with low overfitting average test $\mathrm{OR}_{10}$ values varied from $0.04-0.20$ but only those that were $<0.13$ were selected, average $A U C_{\text {DIFF }}$ varied from $0-0.123$, but only those $<0.05$ were selected. When the models that fit these criteria were projected onto modern climate layers there was little variation between them (Figure 3.7.1). These projections seemed to somewhat represent the contemporary distribution (Figure 3.7.2A). Model 3e2 varied most from the other models (Figure 3.7.1D) and was the most over fit according to $O R_{10}$ metrics $\left(O_{10}=0.130\right)$ (Table 3.7.1). $5 a 15$ had the best discriminatory ability and $A U C_{\text {DIFF }}$ value but also had a high $O R_{10}$ value $\left(O R_{10}=0.129\right)$, while its response curves were flat and uninterpretable. Therefore both models $3 e 2$ and $5 a 15$ were eliminated as choices for the base model. Of the remaining models there was no clear best model but $1 \mathrm{~d} 15$ had the highest discriminatory ability $\left(A \cup C_{\text {train }}=0.874, A \cup C_{\text {test }}=0.872\right)$ and relatively low overfitting metrics $\left(A \cup C_{D I F F}=0.03, \mathrm{OR}_{10}=0.127\right)$. $1 \mathrm{~d} 15$ was therefore chosen as the base model for future predictions. Whichever model was chosen, made little difference to the future predictions. 


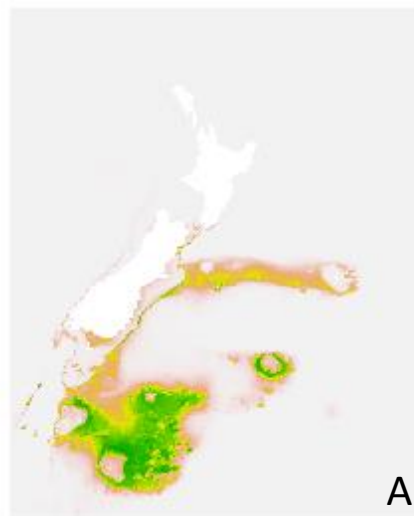

A

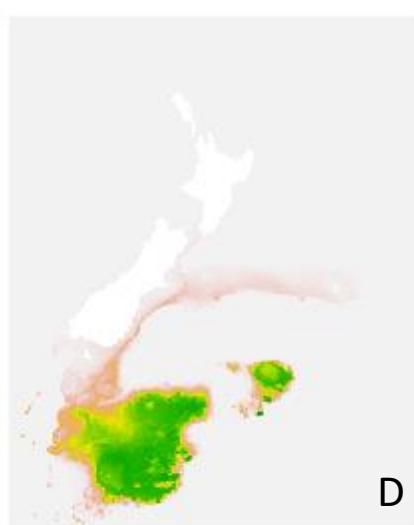

B

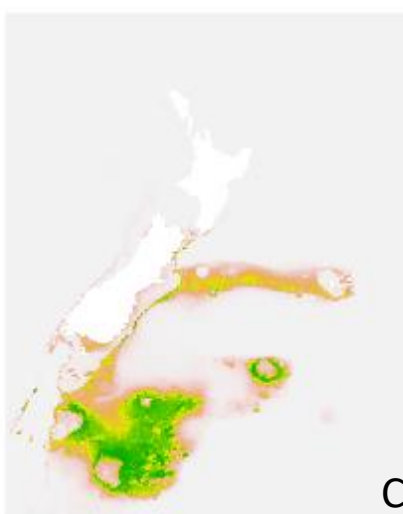

C

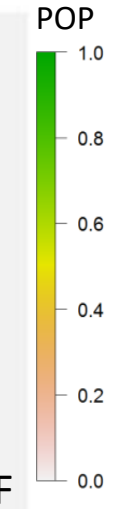

Figure 3.7.1: Southern blue whiting; Six best Maxent models projected on modern climate layers; $1 \mathrm{c} 11$ (A), $1 \mathrm{~d} 15$ (B), 2c11 (C), 3e2 (D), 5a15 (E), 5c9 (F). Colours reflect probability of presence estimates between 0-1. Darker green indicates higher POP and red the reverse. 


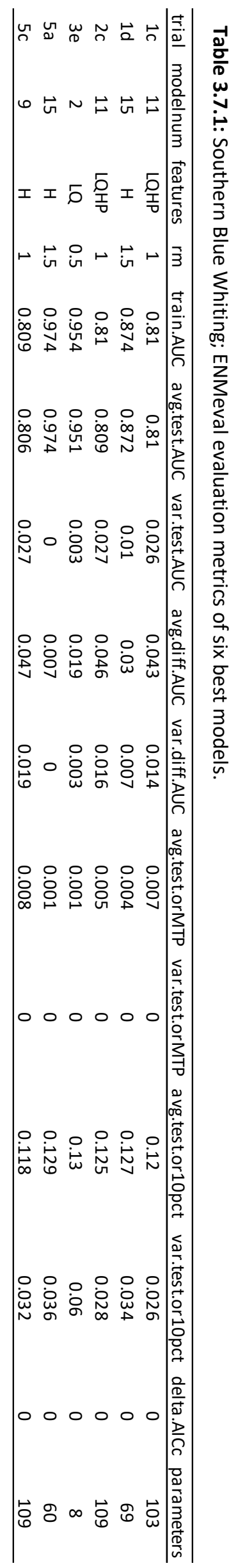




\section{Model Projections}

The 2050 predictions all appeared relatively similar (Figure 3.7.2B-E). The RCPS 8.5 projection had slightly less areas of high POP than the other RCPS predictions. In all scenarios there was a general decline in POP, and this decline was most substantial for areas with high POP. This was reflected in Table 3.7.2, where areas with a POP $>0.9$ decreased by $85.78-100 \%$ and areas with a POP between $0.5-0.6$ decreased by $10.68-36.80 \%$. More areas with highly preferable climatic conditions were lost than areas with less preferable conditions in all RCPS.

The 2050 MESS maps all looked similar (Appendix D.7). All regions around the NI showed high relative uncertainty, while southern regions had high relative certainty, particularly over the Campbell Plateau and Chatham Rise.

\section{$\underline{2100 \text { Model Projections }}$}

The 2100 projections showed more substantial overall decrease in POP than the 2050 predictions, which became more so with scenarios that varied more from modern climates (Figure 3.7.3G-J). On the Campbell Plateau, the main area of contemporary distribution, POP fell from $>0.7$ to $<0.4$ in most areas. Loss of quality habitat was even more prominent in the 2100 predictions than the 2050 predictions as areas with a POP of $>0.9$ either completely or almost disappeared in all scenarios (90.83 - 100\% decrease), while areas with a POP between 0.5 - 0.6 decreased by much less (20.94 66.72\%) (Table 3.7.2).

The 2100 MESS maps were similar to the 2050 MESS maps, but with more relative uncertainty, particularly in the most extreme RCPS 8.5 (Figure 3.7.3B, Appendix D.7). Most areas of high uncertainty were around northern New Zealand, however this extended further southward in the RCPS 8.5. 


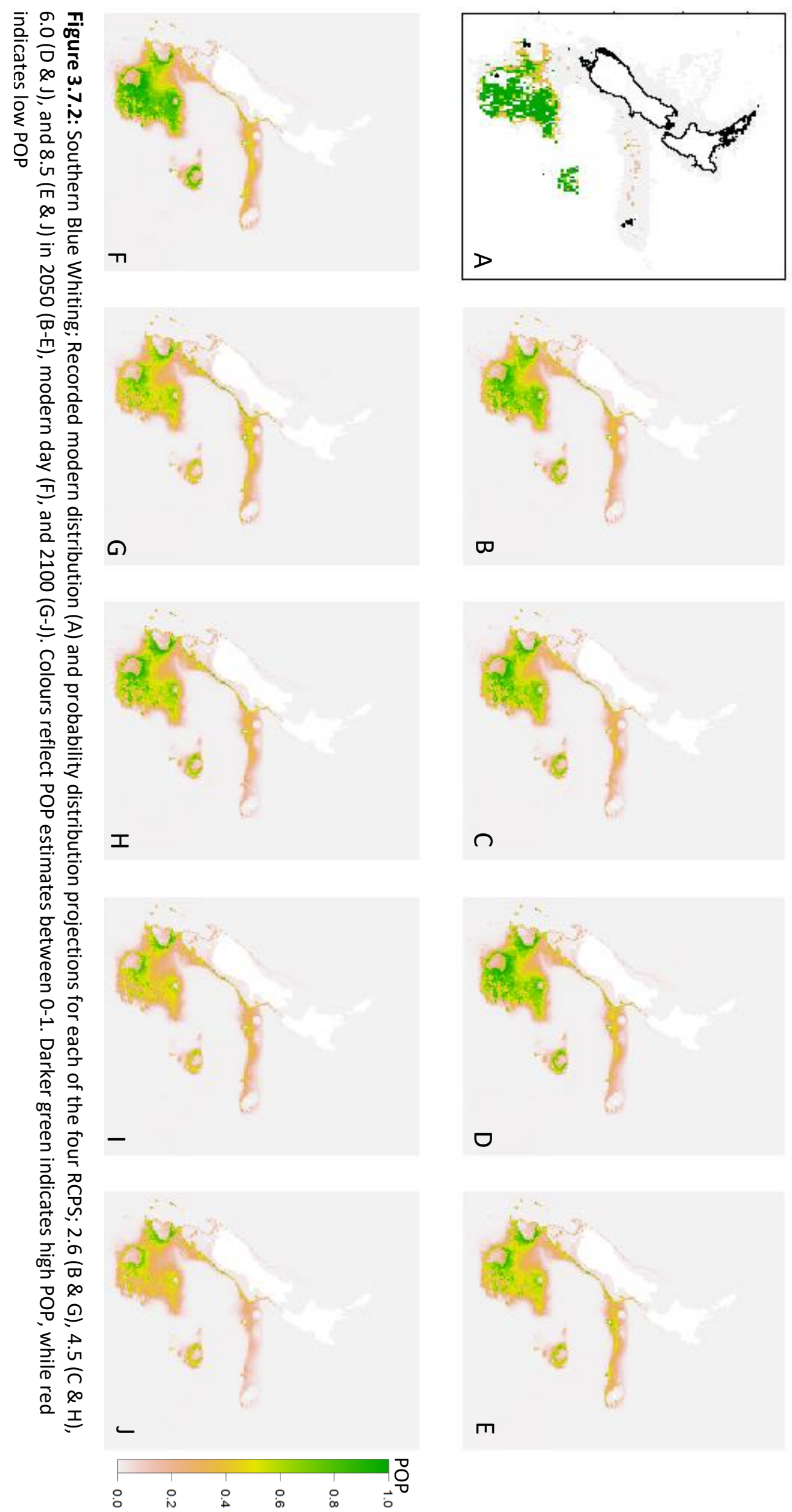


Table 3.7.2: Southern blue whiting; Change in POP values compared to the modern projection for each of the four climate scenarios 2050 and 2100.

\begin{tabular}{lllllllll}
\hline \multicolumn{7}{c}{$\mathbf{2 0 5 0}$} & \multicolumn{5}{c}{$\mathbf{2 1 0 0}$} \\
POP & RCP2.6 & RCP4.5 & RCP6.0 & RCP8.5 & RCP2.6 & RCP4.5 & RCP6.0 & RCP8.5 \\
\hline $\mathbf{0 . 9}$ & -85.784 & -83.006 & -65.804 & -100 & -100 & -90.832 & -100 & -99.655 \\
$\mathbf{0 . 8}$ & -68.011 & -70.841 & -53.78 & -86.208 & -93.175 & -71.272 & -95.681 & -97.952 \\
$\mathbf{0 . 7}$ & -45.847 & -51.391 & -35.737 & -77.711 & -81.157 & -57.701 & -88.134 & -89.782 \\
$\mathbf{0 . 6}$ & -26.464 & -29.37 & -16.978 & -59.36 & -62.526 & -38.331 & -77.416 & -80.173 \\
$\mathbf{0 . 5}$ & -17.091 & -17.653 & -10.68 & -36.801 & -37.44 & -20.944 & -61.039 & -66.715 \\
$\mathbf{0 . 4}$ & -12.977 & -13.108 & -6.815 & -19.762 & -18.802 & -13.693 & -39.485 & -46.808 \\
$\mathbf{0 . 3}$ & -10.119 & -9.422 & -4.46 & -10.66 & -8.937 & -7.381 & -22.695 & -30.975 \\
$\mathbf{0 . 2}$ & -7.532 & -6.794 & -4.504 & -6.663 & -5.292 & -5.833 & -11.669 & -19.753 \\
$\mathbf{0 . 1}$ & -3.694 & -2.971 & -2.261 & -3.524 & -1.704 & -2.31 & -5.48 & -10.041 \\
\hline
\end{tabular}
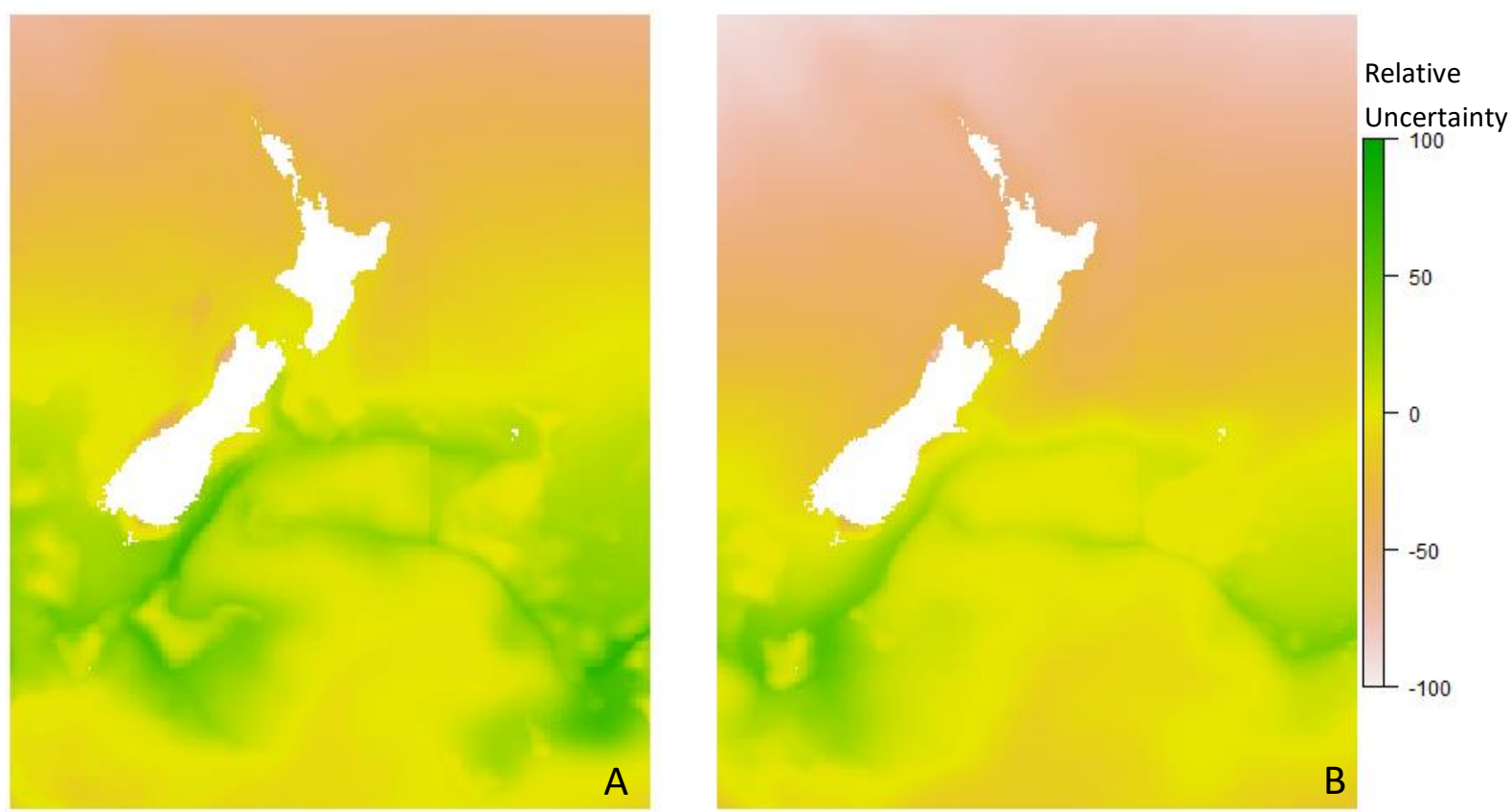

Figure 3.7.3: Southern blue whiting; MESS Maps of RCPS 2.62050 (A) and RCPS 8.52100 (B). MESS maps for all climate scenarios can be found in Appendix D.7. Negative sites indicate most relative uncertainty and positive sites indicate most relative certainty. 


\section{Variable Influence}

The base model predicted that southern blue whiting was absent below $-1000 \mathrm{~m}$ and had increased POP with low SS mean, high SS range, low ST mean, and high ST range (Figure 3.7.4). SS Mean contributed substantially more than other climatic variables to the base model (Figure 3.7.5). A larger range in salinity was not experienced during model training so a constant value was assumed and POP was fixed at a 1.2 degrees range at about 0.8. However, SS range, ST Range, and ST mean contributed very little to the base model (Figure 3.7.5)

The contemporary distribution was most limited by SS Mean throughout most of New Zealand (Figure 3.7.6A). This remained true in the future limiting plot (Figure 3.7.6B), although its influence extended onto the Campbell Plateau. ST Mean also become more of a main limiting factor along the east side of the $\mathrm{SI}$ in the future limiting factor plot.
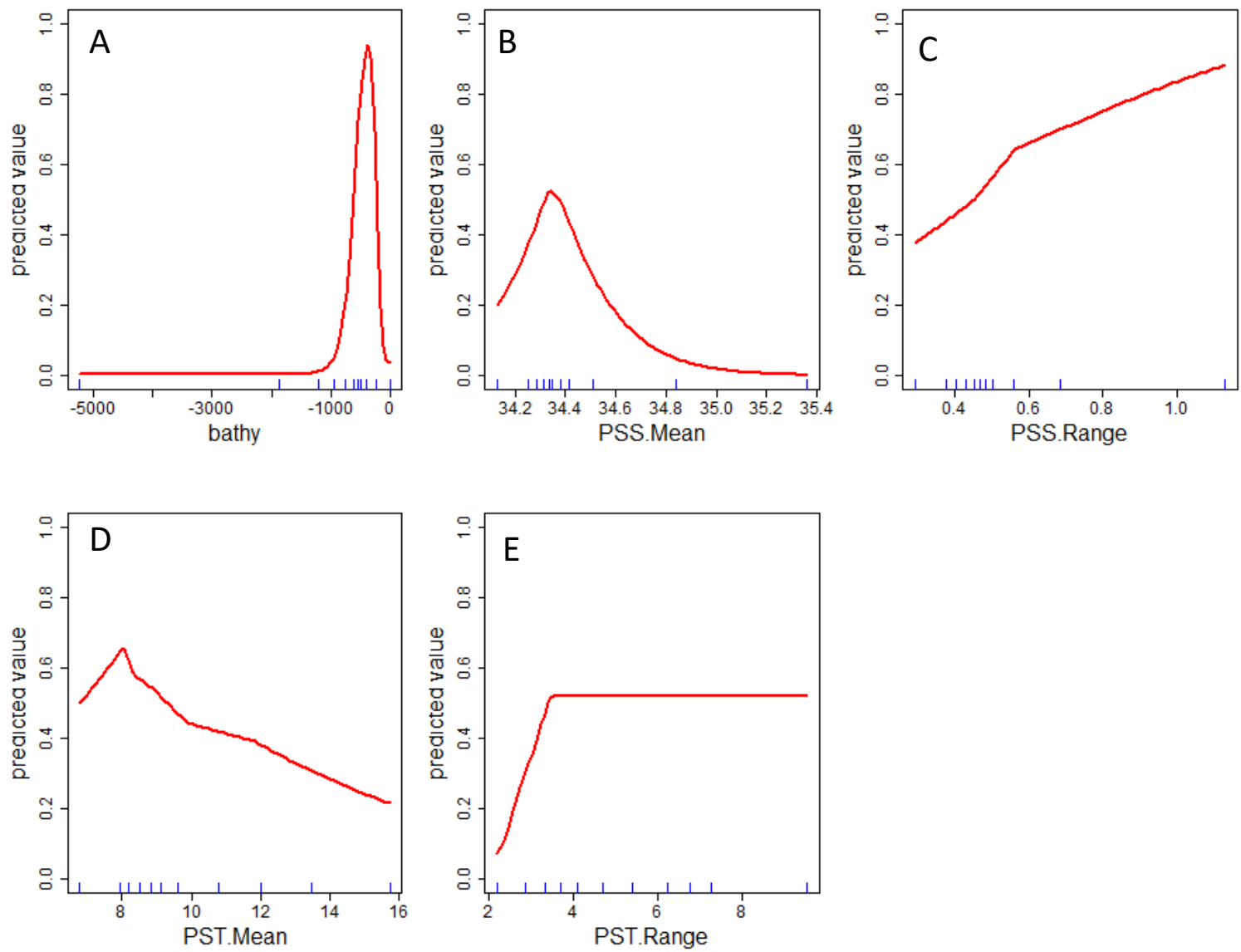

Figure 3.7.4: Southern blue whiting; Predictor response curves indicating how variables used for training affected the base model. Model predictions (red lines) and observations (blue data rug of deciles) are shown. The variables are bathymetry $(A)$, surface salinity mean $(B)$, surface salinity range $(C)$, surface temperature mean (D), and surface temperature range (E). 


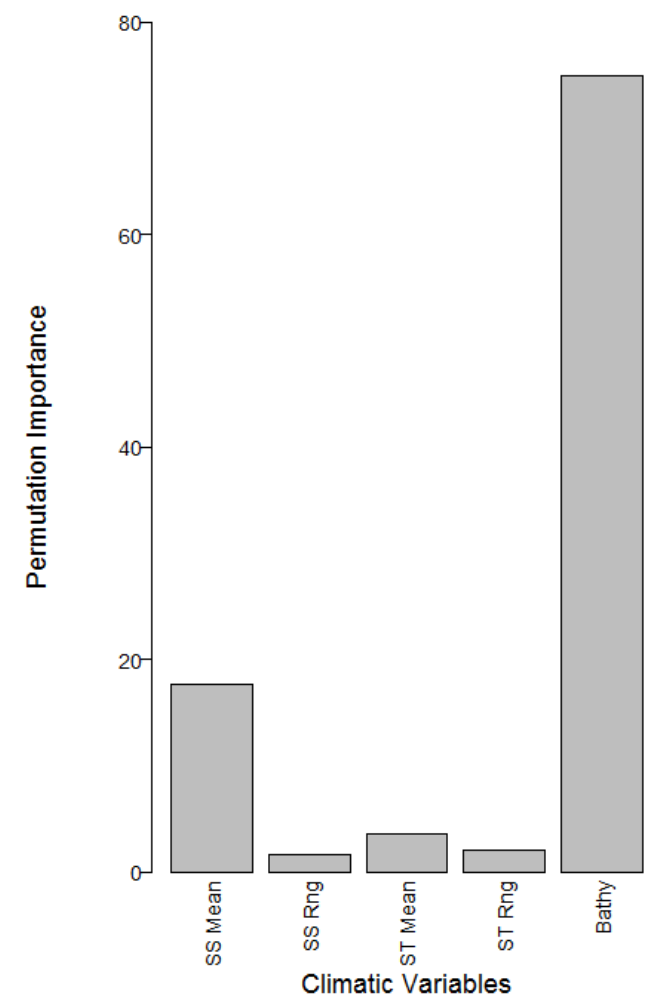

Figure 3.7.5: Southern blue whiting; Predictor variable contribution based on permutation importance (Phillips, 2006). Variable names are shortened here to fit: SS = Surface salinity, ST = Surface temperature, Rng = range, Bathy = bathymetry.
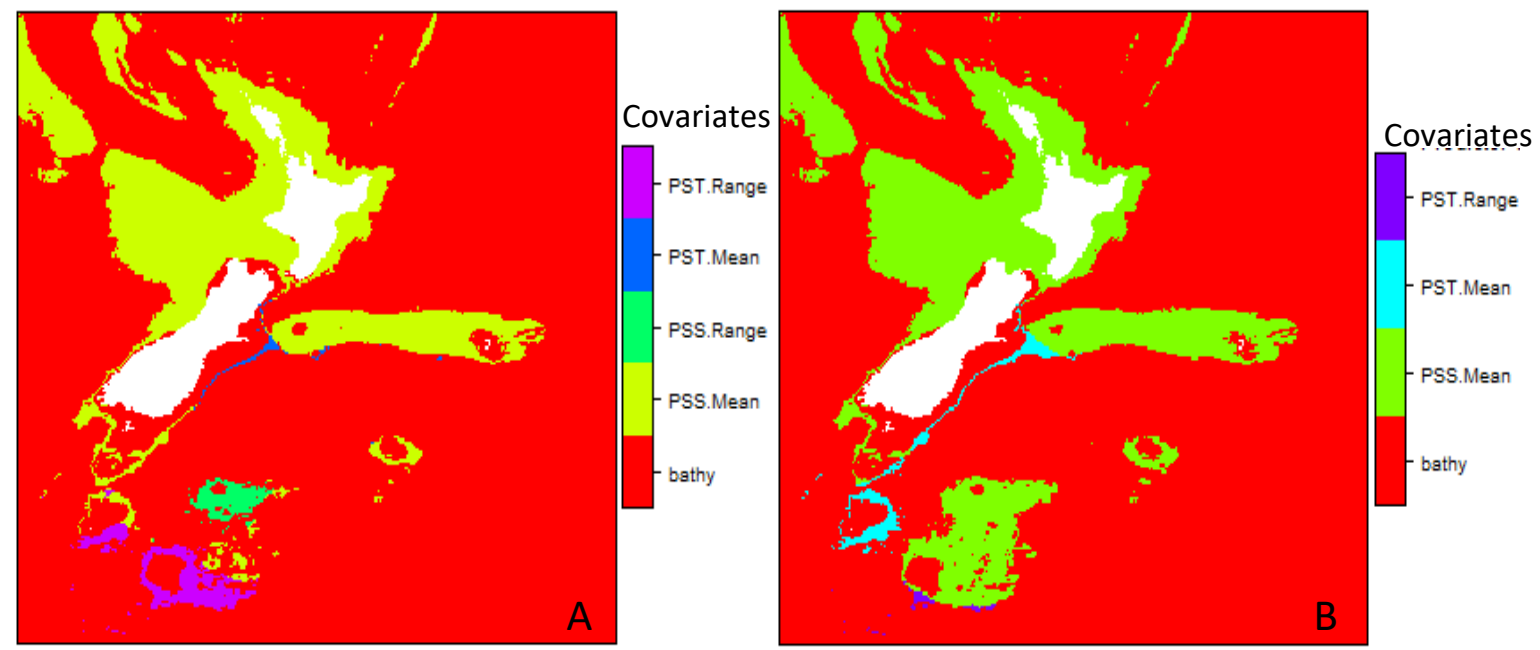

Figure 3.7.6: Southern blue whiting; Limiting factors plots of Modern (A), and RCPS 8.5 in 2100 (A) climates. Colours indicate the most limiting variable to distribution in that area. 


\section{8: HOKI (Macruronus novaezelandiae)}

\section{Model selection}

Of all models generated $\triangle \mathrm{AIC}$ values varied form 0 - 63735.2. Only models with a $\triangle \mathrm{AIC}$ of 0 were selected. Train AUC scores varied from 0.56 - 0.95 and test AUC from 0.55 - 0.94. To select models with good discriminatory ability, only those $>0.75$ were selected. To select models with low overfitting, average test $\mathrm{OR}_{10}$ values varied from $0.07-0.31$ but only those that were $<0.17$ were selected, $A \cup C_{\text {DIFF }}$ varied from $0-0.159$ but only those $<0.05$ were selected. Only the selected values are shown in Table 3.8.1. Of the models that met these criteria the projections for the modern probability distributions were all mostly similar, with the most variation being around Southern New Zealand around the Campbell Plateau (Figure 3.8.1). All modern projections underestimated hoki distribution to varying degrees. All selected models had discriminatory ability values above 0.9 and good AUC DIFF values below 0.009 (Table 3.8.1). Models 4a5, 4a6 and 5a3 all had comparatively lower $\mathrm{OR}_{10}$ overfitting metrics around 0.13 . Of these $4 \mathrm{a} 5$ and $4 \mathrm{a} 6$, which were essentially the same, best matched the hoki contemporary distribution. Model $4 \mathrm{a} 5$ was therefore selected as the base model for all hoki future projections. 

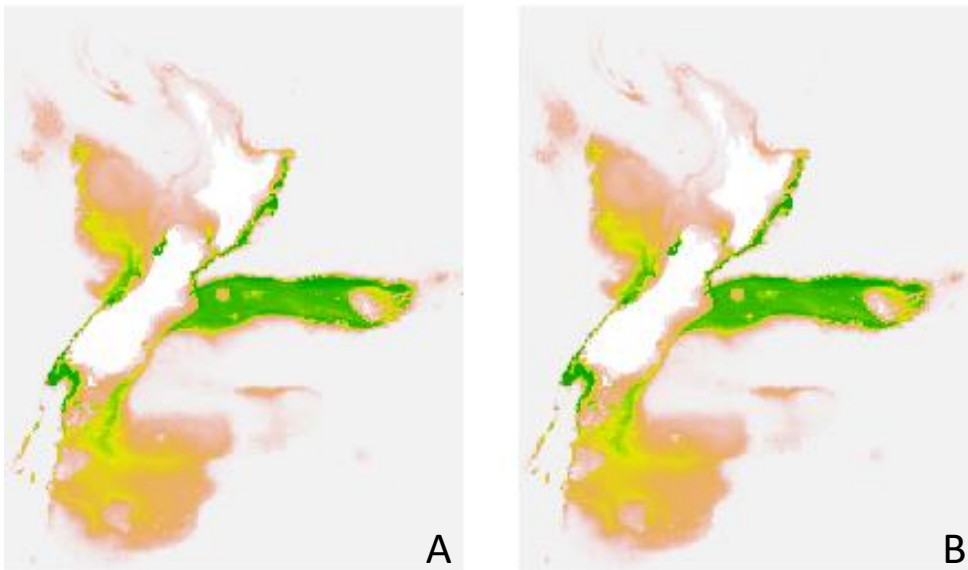

B
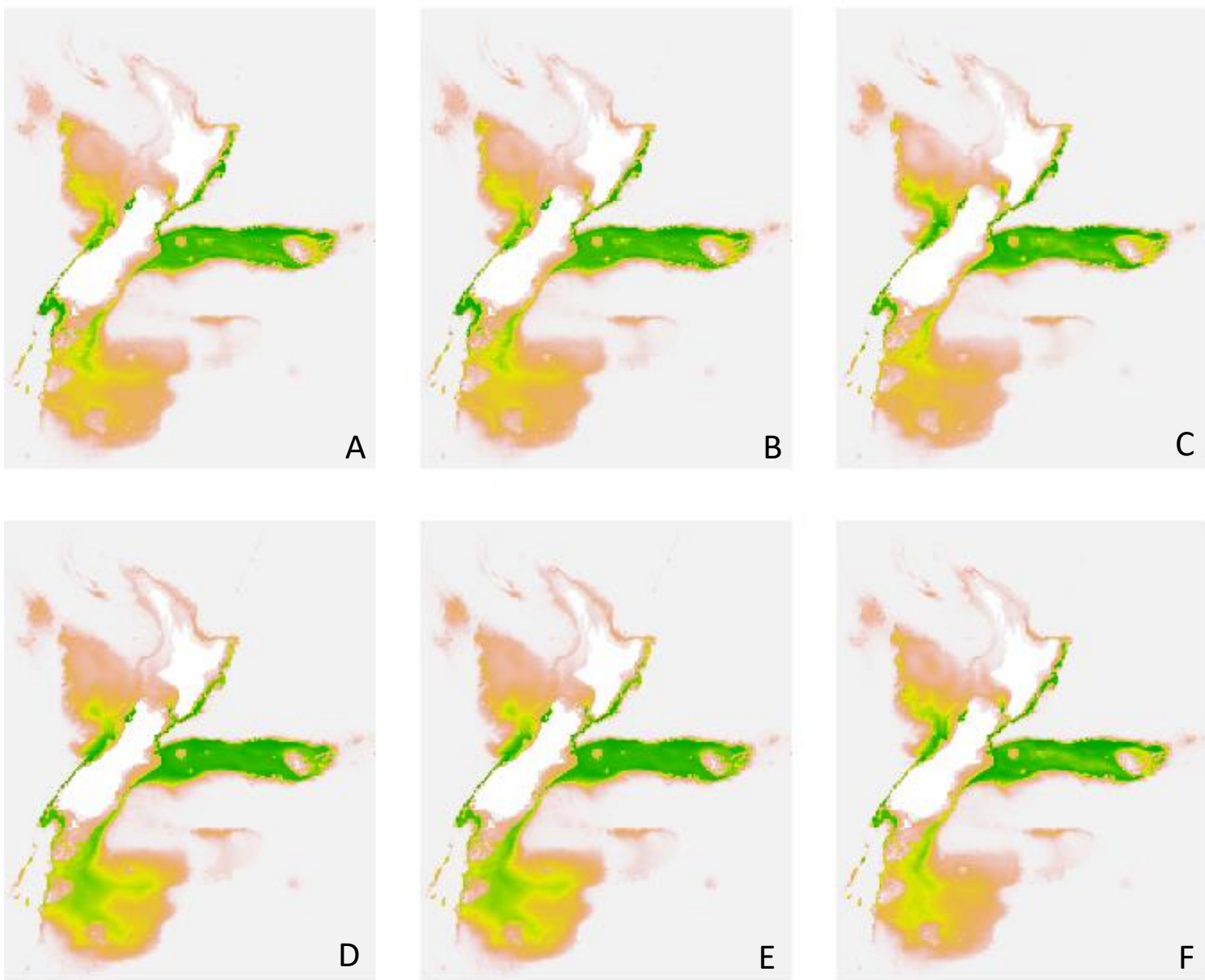

C

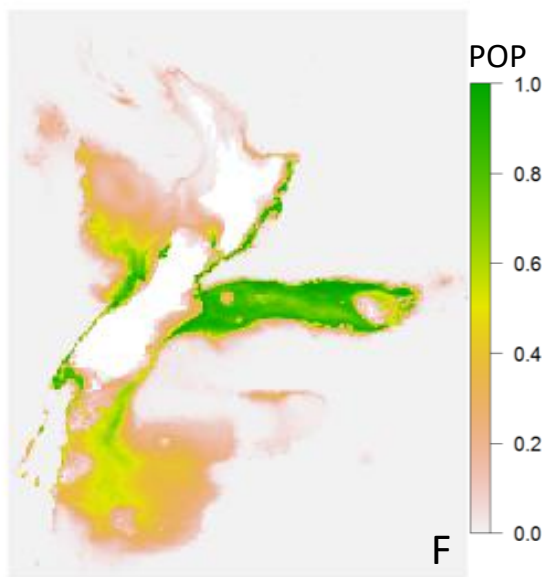

Figure 3.8.1: Hoki; Six best Maxent models projected on modern climate layers; 1a5 (A), 1a6 (B), 2a3 (C), 4a5 (D), $4 \mathrm{a} 6$ (E), $5 \mathrm{a} 3$ (F). Colours reflect POP estimates between 0-1. Darker green indicates higher POP and red the reverse. 


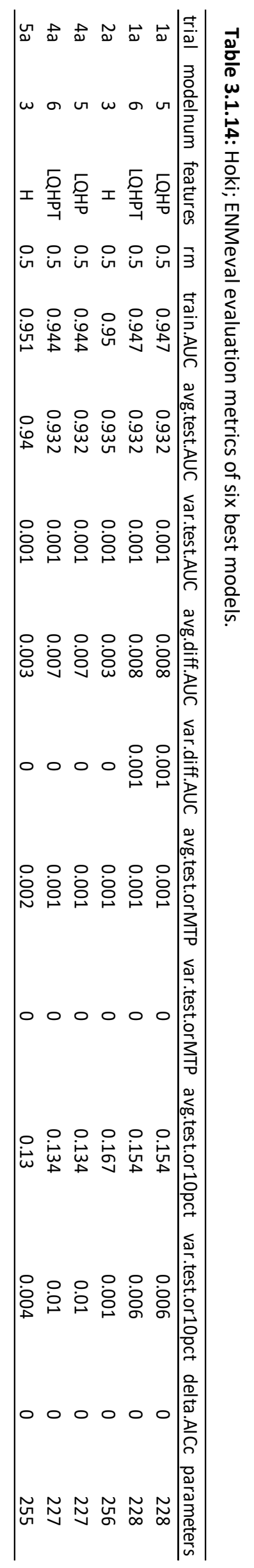




\section{Model Projections}

For the 2050 projections all scenarios were similar (Figure 3.8.2B-E). All scenarios supported maintenance of a high POP for hoki on the Chatham rise, around the east cape of the lower $\mathrm{NI}$, and a thin area around most of the SI and Steward Island. The main difference from the modern projection was on the Campbell plateau, where POP was reduced in all scenarios but most substantially in RCPS 8.5. The overall POP decreased substantially in all scenarios, likely due to the contraction from the Campbell plateau. Areas with a POP of $>0.9$ in RCPS 4.5 and 8.5 decreased by $35.35 \%$ and $28.47 \%$ respectively, while RCPS 2.6 and 6.0 increased by $22.97 \%$ and $24.14 \%$ respectively. Areas with POP between 0.5 and 0.6 decreased by $11.04-24.42 \%$ (Table 3.8.2).

The 2050 MESS maps for all cliamte scenarios displayed very little relative uncertainty (Appendix D.8). Relative certainty was highest around the Chatham rise and south west SI areas.

\section{$\underline{2100 \text { Model Projections }}$}

The 2100 predictions varied more than the 2050 predictions (Figure 3.2G-J). Like in the 2050 predictions, POP decreased in all scenarios and the area of likely distribution decreased, in particular POP on the Campbell Plateau substantially decreased in all scenarios. POP on the Chatham rise also decreased in all scenarios, but most notably so in climate scenarios 6.0 and 8.5. Suitable habitat areas also declined from the lower north island and was limited to the SI in RCPS 6.0 and 8.5. RCP 8.5 showed the most severe contraction of areas with high POP. Green areas showing high POP in the 2100 predictions were limited to the lower Chatham rise, the east coast of the $\mathrm{SI}$ and the lower $\mathrm{SI}$, extending around the Auckland Islands. Areas with highly preferable conditions decreased more than areas with less preferable conditions. This phenomenon was particularly pronounced in RCPS 8.5. For RCP 8.5 , areas with a POP of $>0.9$ were expected to be have almost entirely disappeared, as they decreased by $99.61 \%$ (Table 3.8.2). In all other scenarios, POP $>0.9$ decreased by $14.62-79.8 \%$ and areas with a POP between 0.5-0.6 decreased by $19.63-55.3 \%$.

Most of the 2100 MESS maps also showed very little relative uncertainty, although slightly more was apparent than in the 2050 MESS maps (Appendix D.8). More areas with relatively high uncertainty were observed in RCPS 8.5, than all other climate scenarios, mainly around the upper NI (Figure 3.8.3B). 


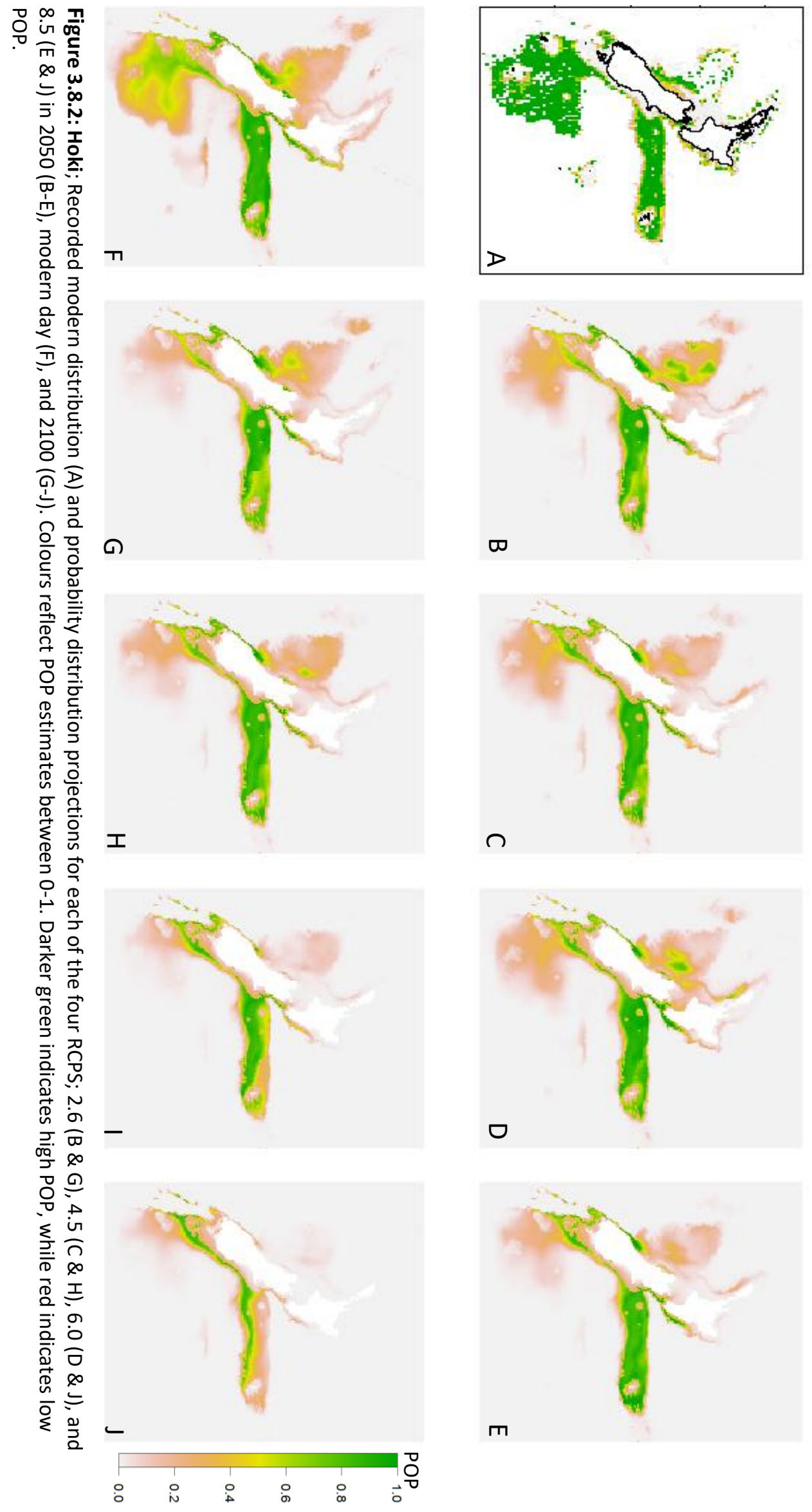


Table 3.8.2: Hoki; Change in POP values compared to the modern projection for each of the four climate scenarios 2050 and 2100 .

\begin{tabular}{lllllllll}
\hline \multicolumn{7}{c}{$\mathbf{2 0 5 0}$} & \multicolumn{5}{c}{$\mathbf{2 1 0 0}$} \\
POP & $\mathbf{R C P 2 . 6}$ & RCP4.5 & RCP6.0 & RCP8.5 & RCP2.6 & RCP4.5 & RCP6.0 & RCP8.5 \\
\hline $\mathbf{0 . 9}$ & 22.972 & -35.352 & 24.139 & -28.473 & -14.62 & -69.183 & -79.803 & -99.611 \\
$\mathbf{0 . 8}$ & -9.156 & -3.525 & -0.817 & 16.522 & -23.883 & -0.241 & -23.77 & -75.542 \\
$\mathbf{0 . 7}$ & 2.62 & 6.602 & 10.904 & 24.371 & -8.446 & 12.737 & -10.319 & -52.233 \\
$\mathbf{0 . 6}$ & -1.51 & -6.23 & -4.241 & 0.941 & -11.604 & -3.977 & -14.95 & -54.303 \\
$\mathbf{0 . 5}$ & -11.038 & -24.415 & -19.182 & -18.752 & -19.628 & -20.123 & -24.771 & -55.3 \\
$\mathbf{0 . 4}$ & -19.792 & -35.128 & -29.185 & -32.23 & -29.472 & -32.648 & -32.101 & -55.519 \\
$\mathbf{0 . 3}$ & -14.247 & -31.847 & -27.024 & -35.596 & -31.29 & -34.1 & -34.01 & -51.873 \\
$\mathbf{0 . 2}$ & -8.521 & -21.199 & -16.73 & -31.38 & -25.093 & -17.505 & -34.519 & -42.095 \\
$\mathbf{0 . 1}$ & -6.333 & -9.778 & -8.542 & -20.055 & -15.549 & -12.457 & -26.441 & -32.342 \\
\hline
\end{tabular}
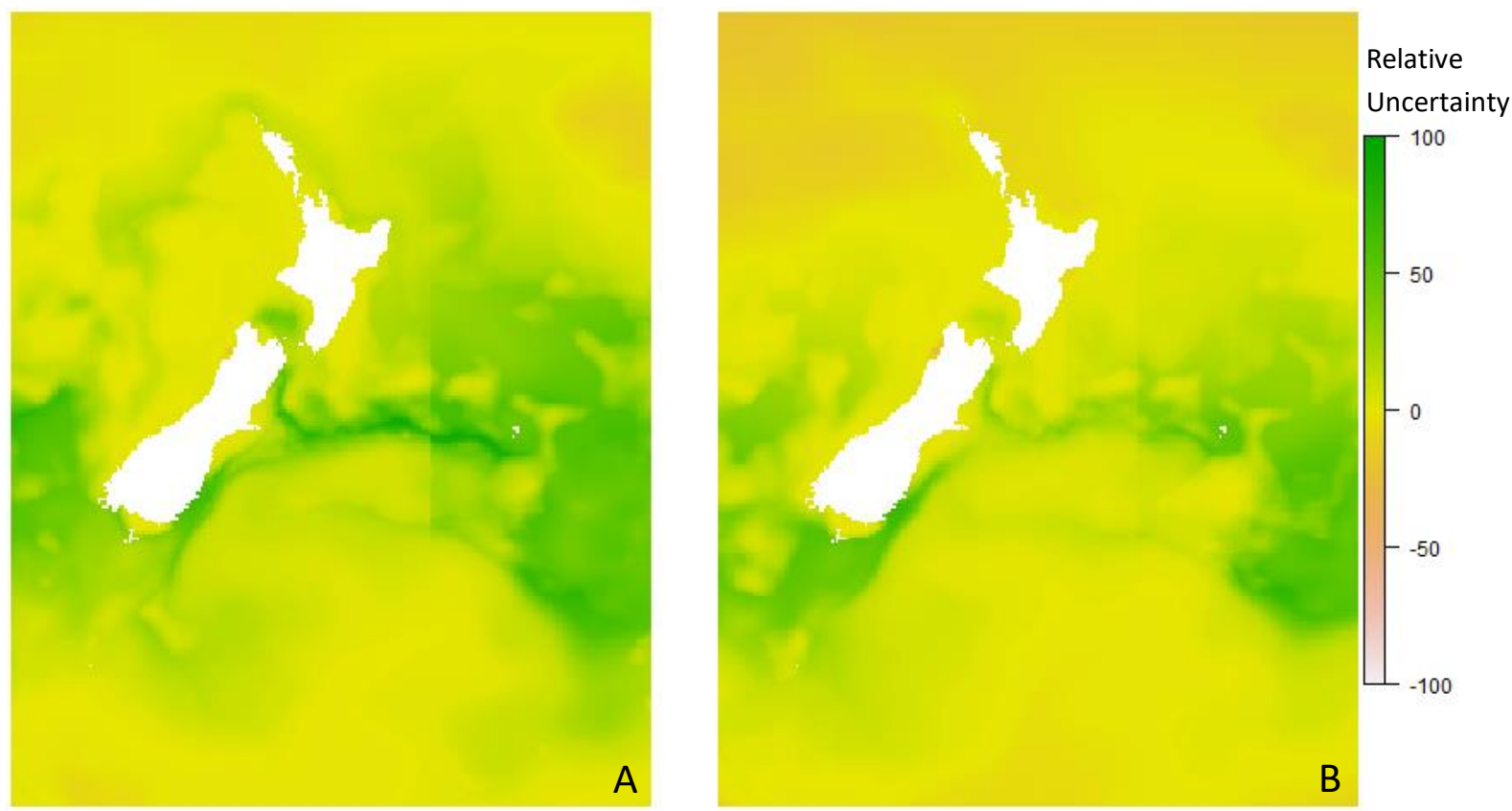

Figure 3.8.3: Hoki; MESS Maps of RCPS 2.6 2050 (A) and RCPS 8.52100 (B). MESS maps for all climate scenarios can be found in Appendix D.8. Negative sites indicate most relative uncertainty and positive sites indicate most relative certainty. 


\section{Variable Influence}

The base model predicted that hoki were absent depths below $-2000 \mathrm{~m}$, and had increased POP with high SS mean, and ST range (Figure 3.8.4). ST mean seemed to negatively affect POP. The effect of ST Range and ST mean seem at least partially implausible, so the temperature effect is difficult to interpret. Mean ST effect seems plausible from $\sim 10-20^{\circ} \mathrm{C}$ but at lower temperatures its effect is more ambiguous. SS range had almost no effect. ST mean had the highest permutation importance of all climatic variables, followed by SS mean (Figure 3.8.5). ST range and SS range both had a permutation importance of almost 0 .

The main variable limiting contemporary distribution was ST mean however around the Campbell Plateau this was SS mean and further southward was ST range (Figure 3.8.6). This shifted slightly under RCPS 8.5, where SS mean limited distribution further southward and around the Campbell Plateau, while ST mean was the main limiting factor in more area around northern New Zealand, the West Coast of the SI and the Chatham Rise.
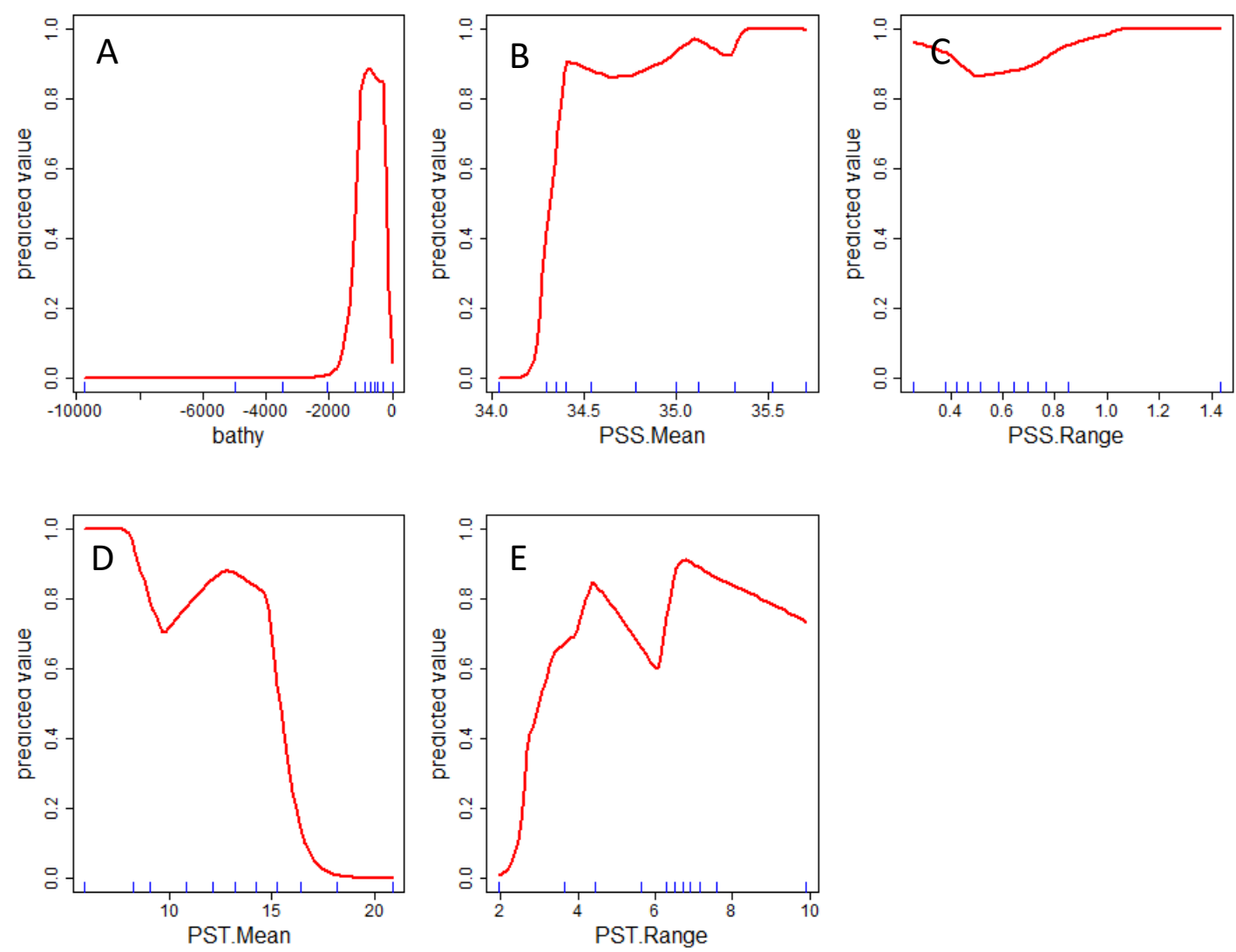

Figure 3.8.4: Hoki; Predictor response curves indicating how variables used for training affected the base model. Model predictions (red lines) and observations (blue data rug of deciles) are shown. The variables are bathymetry $(A)$, surface salinity mean $(B)$, surface salinity range $(C)$, surface temperature mean $(D)$, and surface temperature range $(E)$. 


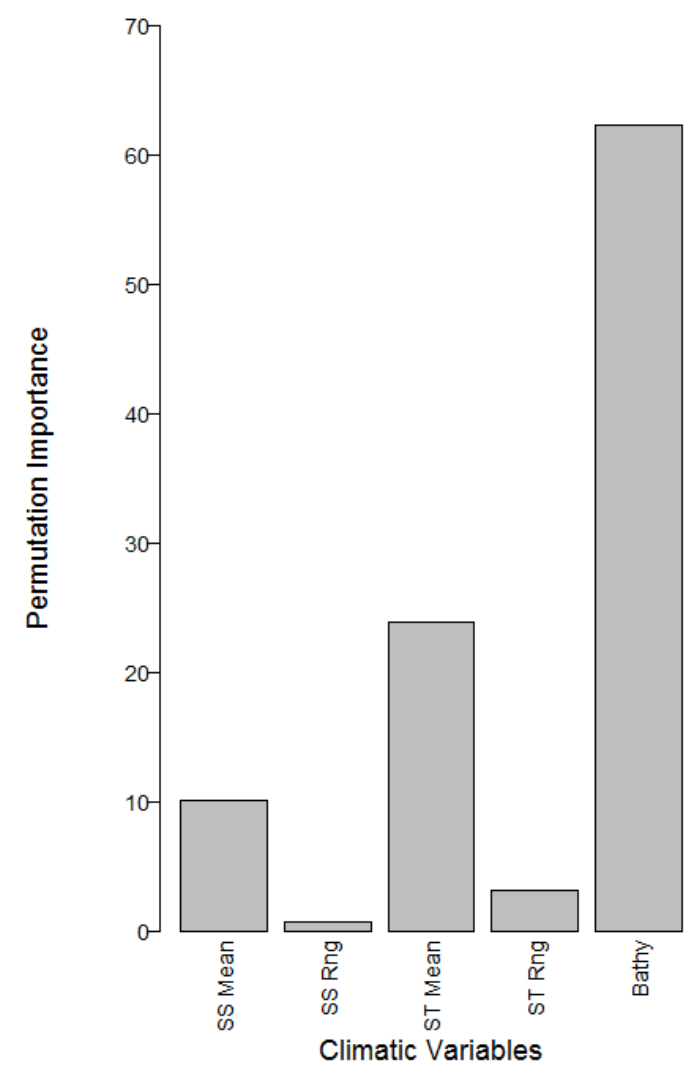

Figure 3.8.5: Hoki; Predictor variable contribution based on permutation importance (Phillips, 2006). Variable names are shortened here to fit: SS = Surface salinity, ST = Surface temperature, Rng = range, Bathy = bathymetry.

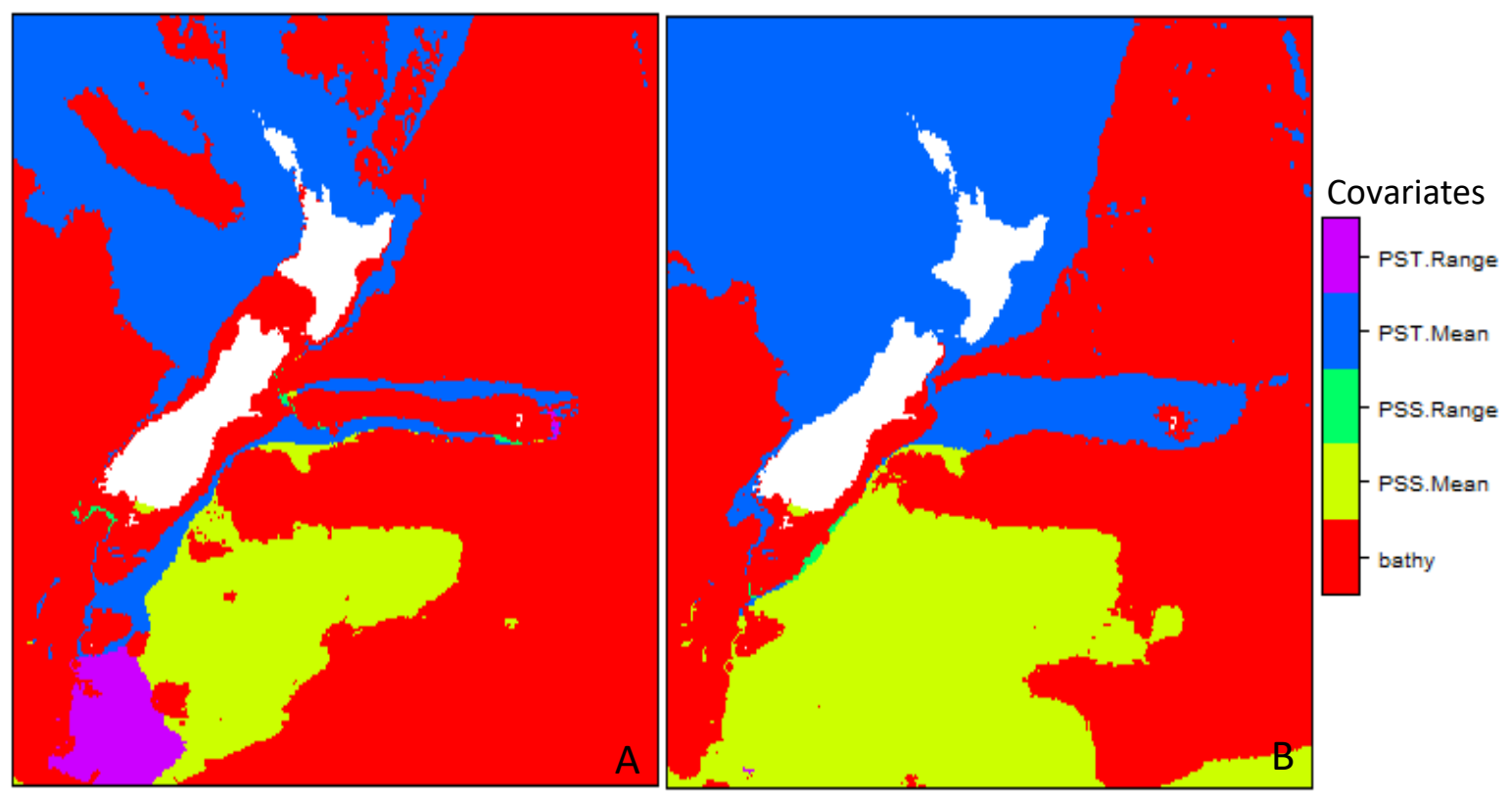

Figure 3.8.6: Hoki; Limiting factors plots of Modern (A), and RCPS 8.5 in 2100 (A) climates. Colours indicate the most limiting variable to distribution in that area. 


\section{9: LING (Genypterus blacodes)}

\section{$\underline{\text { Model Selection }}$}

Of all models generated $\triangle A I C$ values varied form 0 - 33933.65. Only models with a $\triangle A I C$ of 0 were selected. Train AUC scores varied from 0.55 - 0.95 and test AUC from 0.54 - 0.95. To select models with good discriminatory ability only those $>0.75$ were selected. To select models with low overfitting, average test $\mathrm{OR}_{10}$ values varied from $0.04-0.35$ but only those that were $<0.15$ were selected, average $A \cup C_{\text {DIFF }}$ varied from $0-0.17$ but only those $<0.05$ were selected. Models selected are shown in table 3.9.1. Model 1a15 substantially under predicted ling presence on the Campbell Plateau and over predicted around the north of the SI (Figure 3.9.1A), while model 3e22 under predicted ling presence on the Chatham rise and the northern SI (Figure 3.9.1F). Models 3c5 and $3 e 22$ had discriminatory abilities that only just surpassed the acceptable discriminatory ability threshold of $>0.75$ (Table 3.9.1). Of the remaining models, $3 a 9,3 d 5$ and $3 d 6$, all fit the contemporary known distribution reasonably well, although possibly slightly under predicted in southern regions (Figure 3.9.1B,C\&D). 3a9 had better discriminatory ability $\left(A \cup C_{\text {train }}=0.94, A \cup C_{\text {test }}=0.94\right)$ and lower over fitting $\left(A \cup C_{D I F F}=0.006, \mathrm{OR}_{10}=0.105\right)$, so model 3a9 was selected as the base model. 

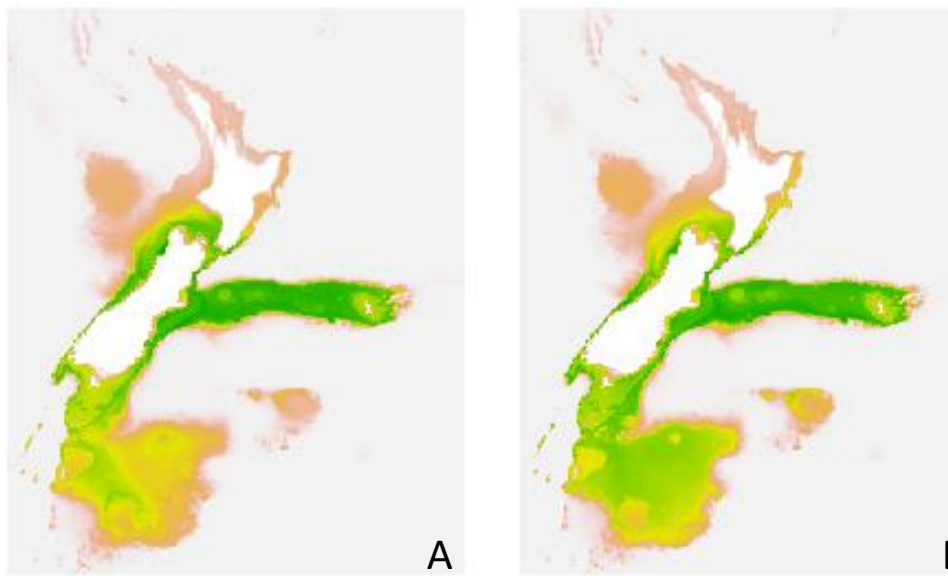

B
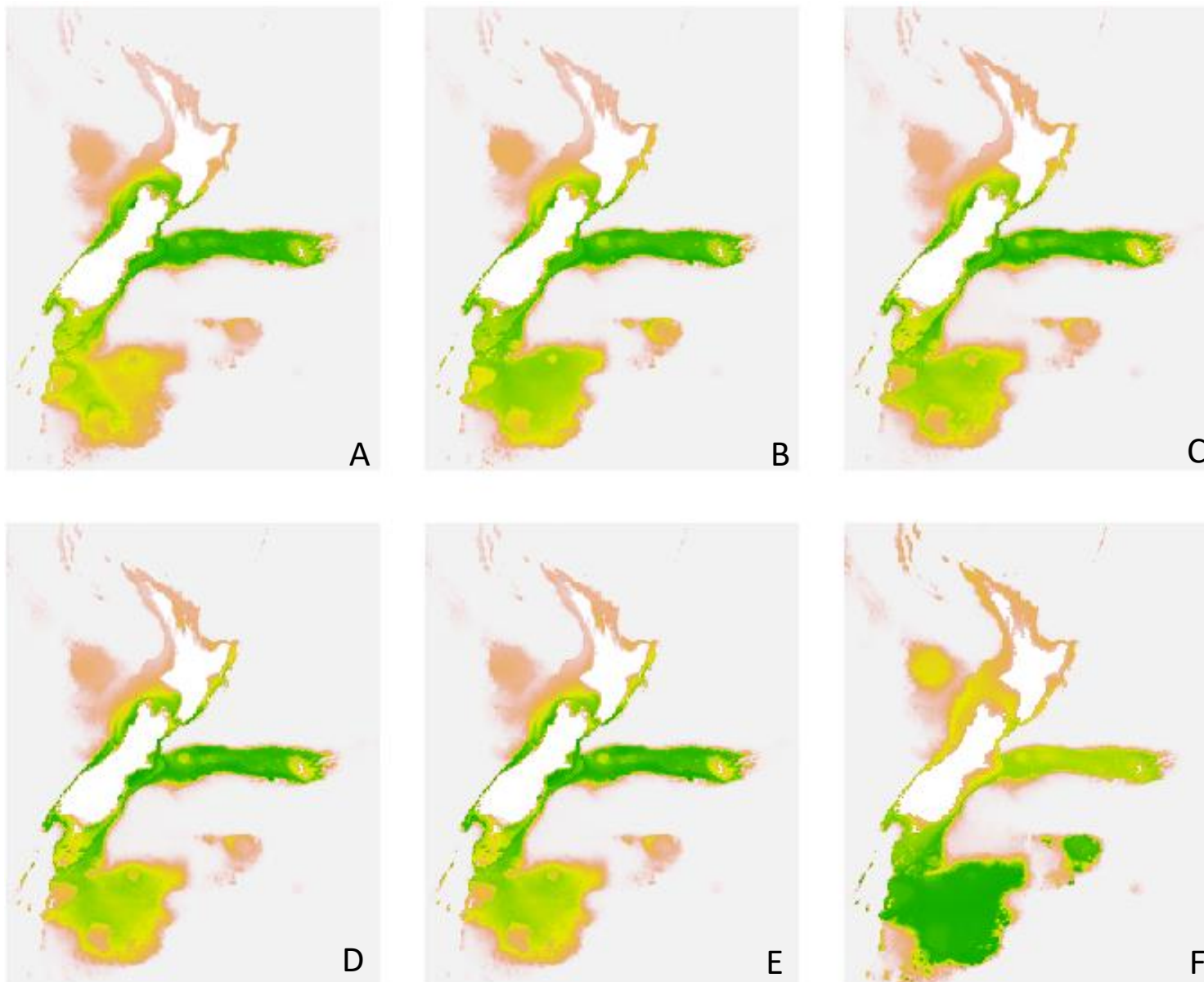

C

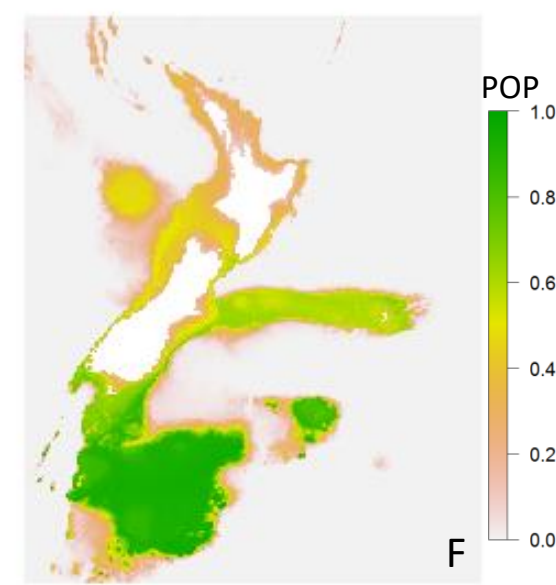

Figure 3.9.1: Ling; Six best Maxent models projected on modern climate layers; $1 \mathrm{a} 15$ (A), 3a9 (B), 3c5 (C), 3d5 (D), 3d6 (E), 3e22 (F). Colours reflect probability of presence estimates between 0-1. Darker green indicates higher POP and red the reverse. 


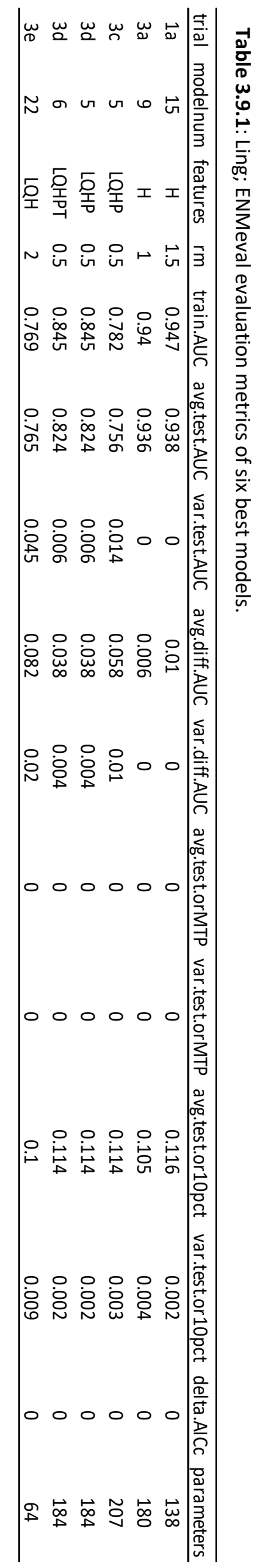




\section{Model Projections}

The 2050 projections showed the reduction of quality habitat for ling, particularly on the Campbell Plateau, the N/W regions around the SI and Cook Strait (Figure 3.9.2B-E). The Chatham Rise was a stable area of high POP in every RCPS in 2050. Reduction of area in all POP bins below 0.6 but increase in all above 0.7 in RCPS 8.5, suggested the amount of suitable areas decreased but the areas with high POP increased (Table 3.9.2). Areas with a POP $>0.9$ decreased by $23.52-42.10 \%$, with the exception of RCPS 8.5 which increased by $45.97 \%$. Over all scenarios, areas with a POP between 0.5 -0.6 decreased by $20.25-33.91 \%$.

2050 MESS maps suggested low levels of relative uncertainty in all RCPS (Appendix D.9). Highest certainty could be observed around the Chatham Rise and S/W regions of the SI in RCPS 2.6 (Figure 3.9.3A).

$\underline{2100}$

The RCPS 6.0 and 8.5 predicted almost complete loss of suitable habitat from west coast of $\mathrm{SI}$, and substantial reduction on the Campbell Plateau in all scenarios similar to that in the 2050 predictions (Figure 3.9.2G-J). In the RCPS 8.52100 prediction, POP on the Chatham rise has decreased so that high quality conditions for ling only occupied the southern half of the Chatham Rise. These projections had more variation between each RCPS than the 2050 projections. Areas with a POP $>0.9$ decreased by $18.93-53.18 \%$, Areas with a POP between $0.5-0.6$ decreased by $32.45-49.58 \%$.

2100 MESS maps also showed little relative uncertainty (Appendix D.9). Uncertainty was most evident in RCPS 8.5 (Figure 3.9.3B). In this scenario regions towards the north of the $\mathrm{NI}$ showed the most relative uncertainty. 

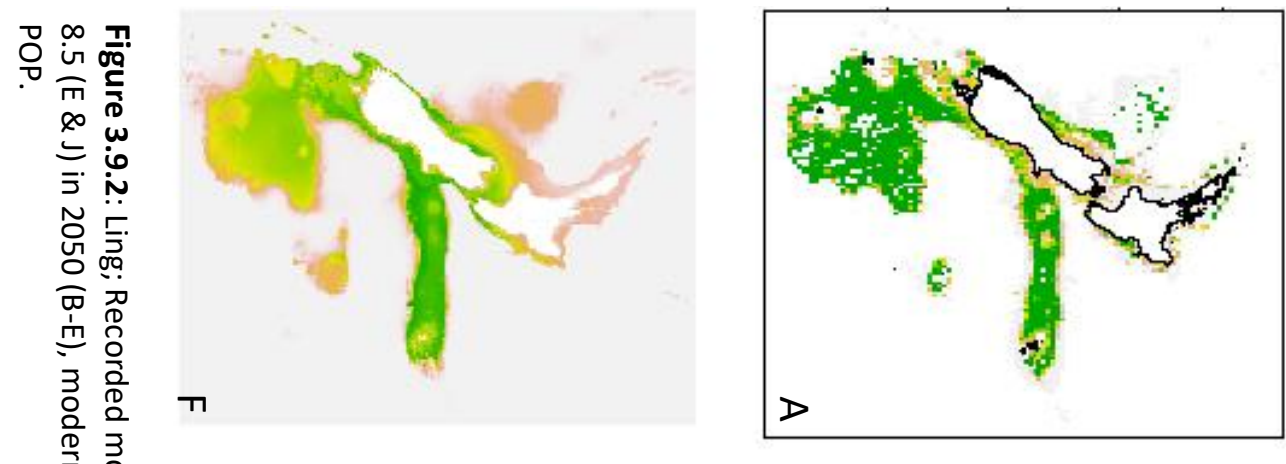

윽

足 $\frac{2}{2}$

끄

ํㅡㄹ

눙 웅

올

을

응 웅 $\square$

ज

蛋

号 음
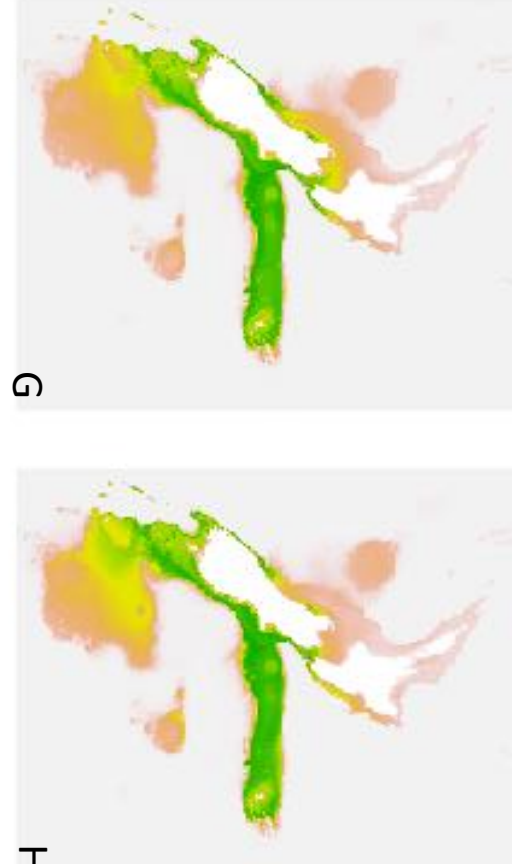

D

芦

궁 응

它

뭉

齐

욤

육 으

근

帤

号

츨 균

吕

象

둥

응

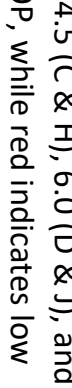

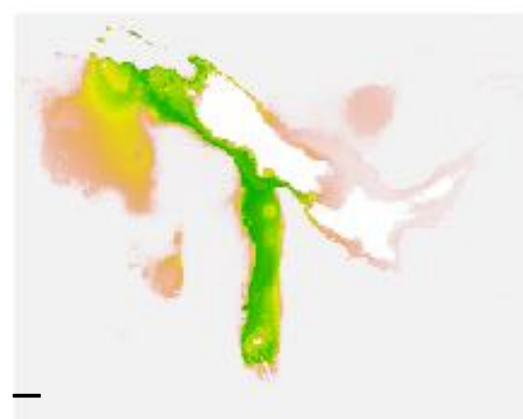

$\infty$
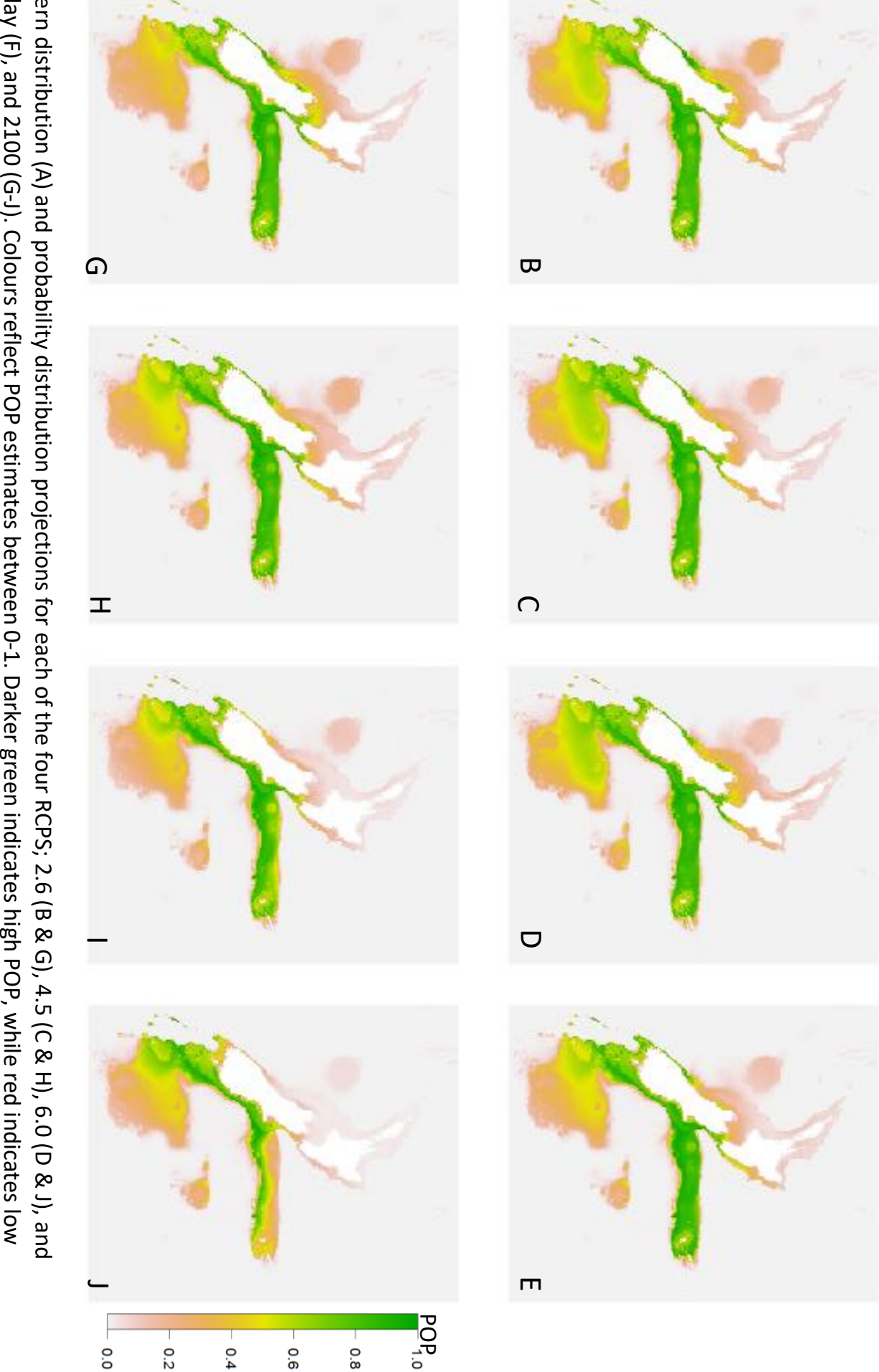
Table 3.9.2: Ling; Change in POP values compared to the modern projection for each of the four climate scenarios 2050 and 2100 .

\begin{tabular}{lllllllll}
\hline \multicolumn{7}{c}{$\mathbf{2 0 5 0}$} \\
$\mathbf{P O P}$ & $\mathbf{R C P 2 . 6}$ & $\mathbf{R C P 4 . 5}$ & $\mathbf{R C P 6 . 0}$ & $\mathbf{R C P 8 . 5}$ & $\mathbf{R C P 2 . 6}$ & $\mathbf{R C P 4 . 5}$ & RCP6.0 & RCP8.5 \\
\hline $\mathbf{0 . 9}$ & -40.196 & -23.524 & -42.101 & 45.97 & -18.928 & -21.385 & -20.686 & -53.183 \\
$\mathbf{0 . 8}$ & -1.852 & 8.499 & 13.531 & 14.619 & -8.067 & 4.378 & -18.421 & -58.138 \\
$\mathbf{0 . 7}$ & -0.3 & 5.098 & 9.17 & 9.45 & 1.987 & 1.301 & -11.261 & -42.533 \\
$\mathbf{0 . 6}$ & -33.01 & -21.049 & -22.684 & -28.998 & -33.604 & -29.882 & -34.621 & -51.148 \\
$\mathbf{0 . 5}$ & -25.187 & -20.352 & -20.248 & -33.912 & -40.869 & -32.453 & -37.837 & -49.579 \\
$\mathbf{0 . 4}$ & -18.691 & -18.821 & -17.109 & -26.202 & -33.173 & -25.948 & -31.636 & -37.682 \\
$\mathbf{0 . 3}$ & -11.053 & -13.055 & -8.585 & -20.873 & -22.236 & -19.499 & -27.191 & -27.847 \\
$\mathbf{0 . 2}$ & -4.375 & -11.964 & -8.041 & -14.443 & -11.783 & -11.897 & -22.379 & -19.697 \\
$\mathbf{0 . 1}$ & -6.551 & -10.484 & -6.313 & -11.744 & -7.177 & -8.759 & -17.191 & -20.731 \\
\hline
\end{tabular}
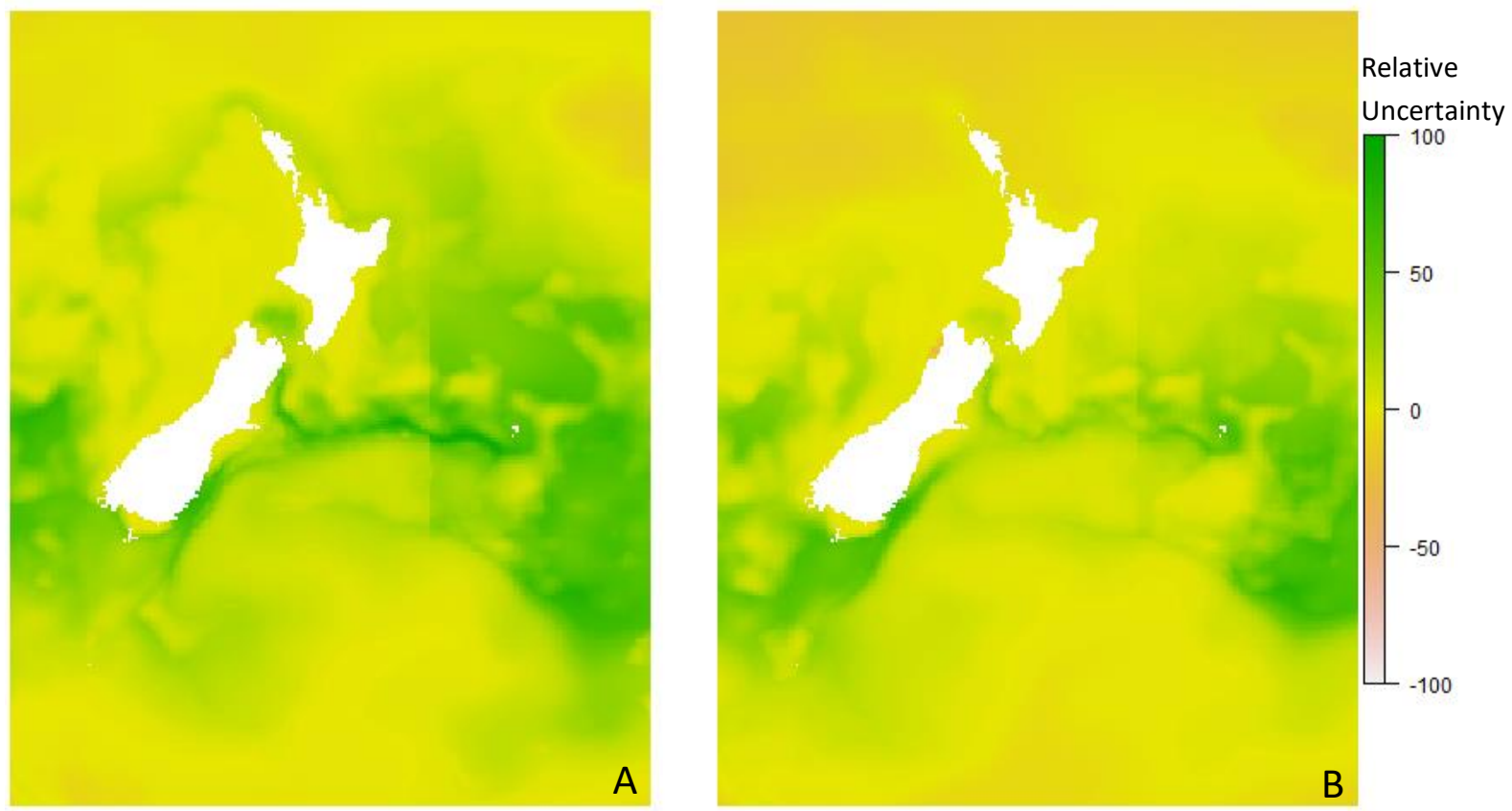

Figure 3.9.3: Ling; MESS Maps of RCPS 2.6 2050 (A) and RCPS 8.52100 (B). MESS maps for all climate scenarios can be found in Appendix D.9. Negative sites indicate most relative uncertainty and positive sites indicate most relative certainty. 


\section{Variable Influence}

The base model predicted that ling were absent below $-2000 \mathrm{~m}$ and had increased POP with mean SS, SS range, and ST range (Figure 3.9.4). The base model also predicted a slight preference for cooler temperatures, although information below $5^{\circ} \mathrm{C}$ was outside of the range of the training data so a constant value was assumed and POP was fixed at 5 degrees at about 0.3. The effect of ST Mean and SS mean seem at least partially implausible and their effects are difficult to interpret. Mean ST effect seemed plausible from approximately $10-15^{\circ} \mathrm{C}$ but at lower temperatures its effect is more cryptic. Mean SS effect seemed plausible from about $34-35.4 p s u$ but at higher salinity shoots up in a way that is likely not representative of the actual true salinity effect. All climatic variables had limited contribution to the base model other than ST mean (Figure 3.9.5).

ST mean was the main limiting climatic variable throughout New Zealand waters in the contemporary climate (Figure 3.9.6A). One notable exception was on the Chatham Rise where SS Range was the main limiting factor. In the future limiting factor plot, ST mean remained the main limiting factor throughout most of New Zealand, with the exception of on and around the Campbell Plateau, where SS mean has become more limiting (figure 3.9.6B). The limiting factor on the Chatham Rise shifted to mean temperature in the future climate.
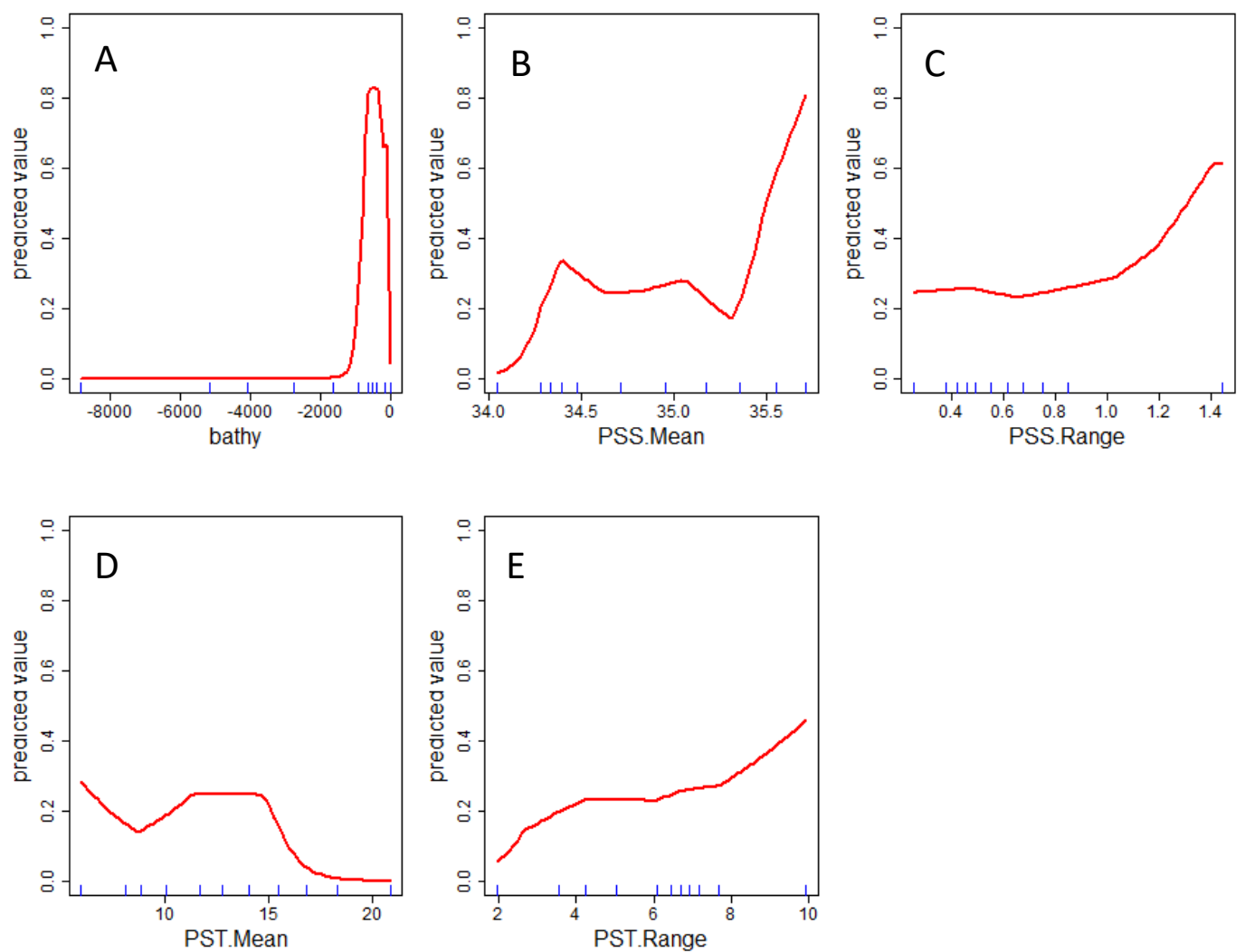

Figure 3.9.4: Ling; Predictor response curves indicating how variables used for training affected the base model. Model predictions (red lines) and observations (blue data rug of deciles) are shown. The variables are bathymetry $(A)$, surface salinity mean $(B)$, surface salinity range $(C)$, surface temperature mean $(D)$, and surface temperature range $(E)$. 


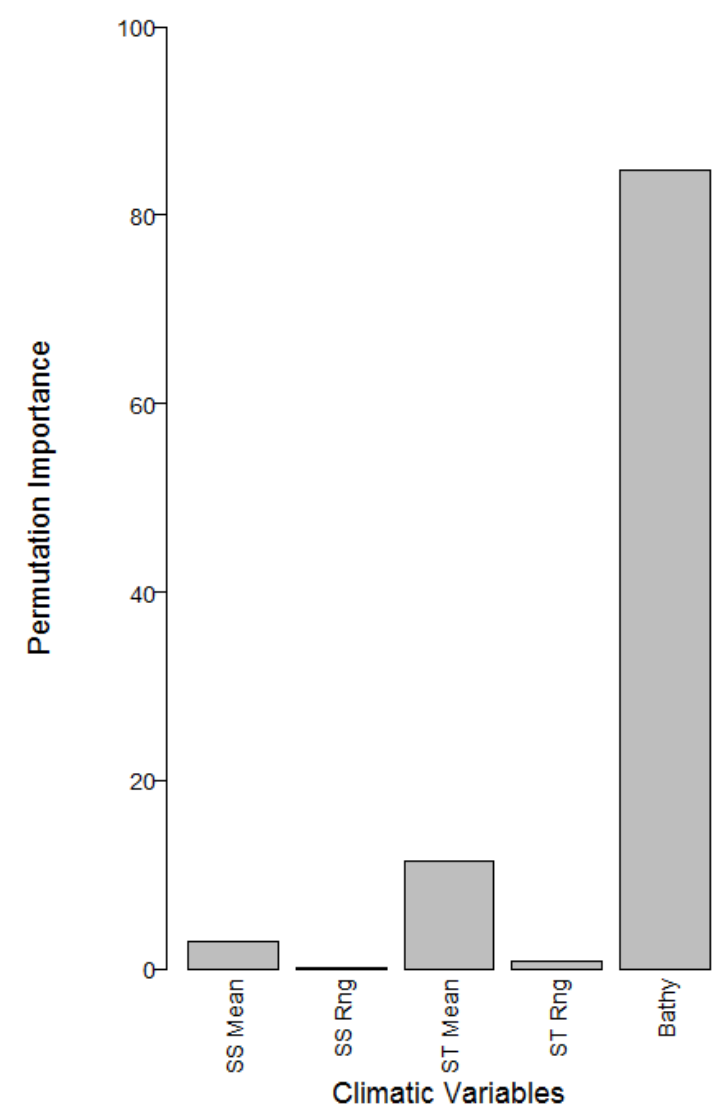

Figure 3.9.5: Ling; Predictor variable contribution based on permutation importance (Phillips, 2006). Variable names are shortened here to fit: SS = Surface salinity, ST = Surface temperature, Rng = range, Bathy = bathymetry.
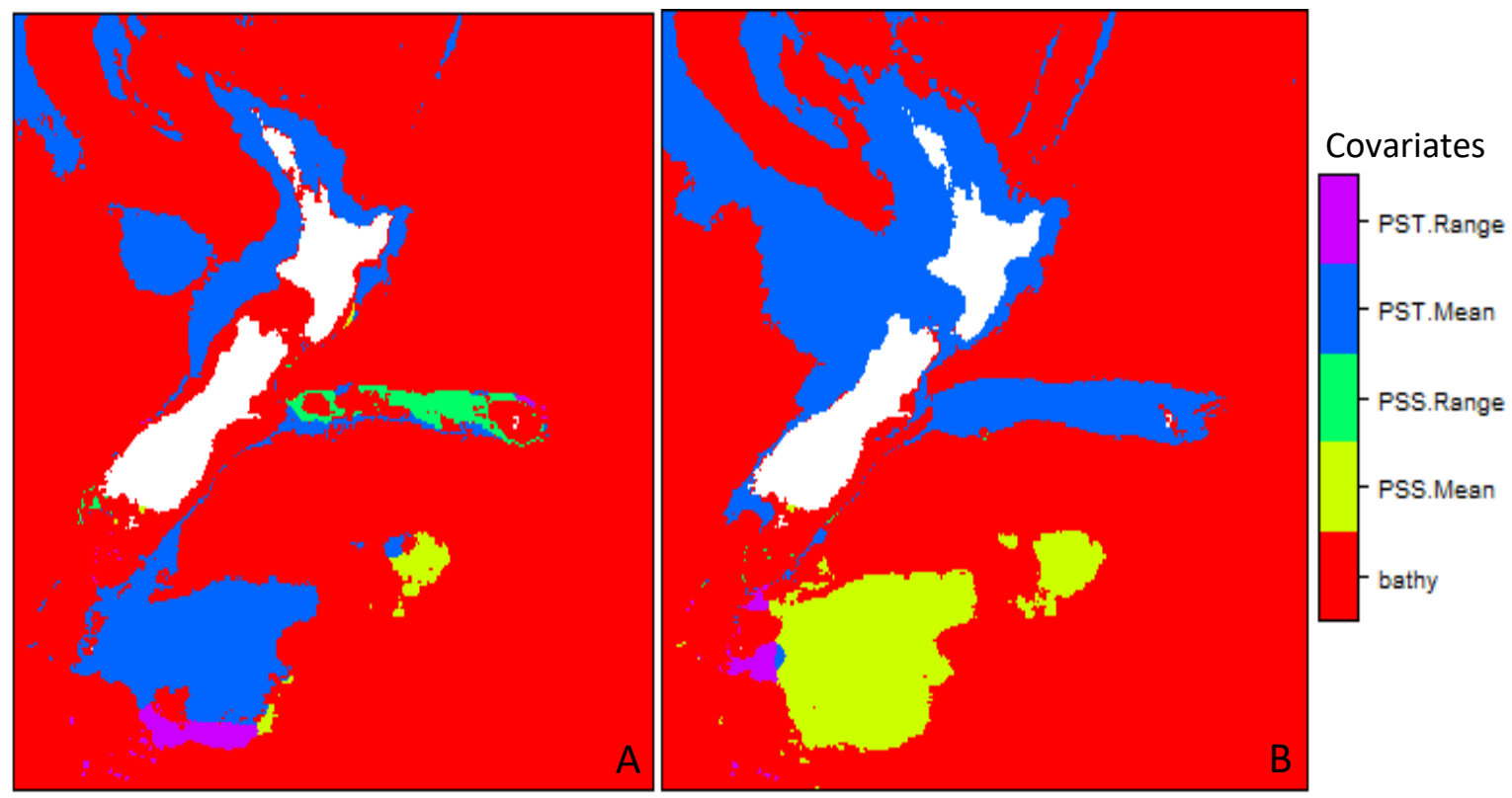

Figure 3.9.6: Ling; Limiting factors plots of modern (A), and RCPS 8.5 in 2100 (A) climates. Colours indicate the most limiting variable to distribution in that area. 


\subsection{0: New Zealand ARROW SQUID (Nototodarus sloanii)}

\section{Model Selection}

Models selected are shown in Table 3.10.1. $\Delta$ AIC values varied form 0 - 12799.16, only models with a $\triangle A I C$ of 0 were selected. Train AUC scores varied from 0.59 - 0.97 and test AUC from $0.57-0.97$. To select models with good discriminatory ability only those $>0.75$ were selected. To select models with low overfitting average test $\mathrm{OR}_{10}$ values varied from $0.04-0.21$ but only those that were $<0.15$ were selected, average $A \cup C_{\text {DIFF }}$ varied from $0-0.097$ but only those $<0.05$ were selected. Of the models that fit these criteria, all projections onto modern climate layers looked similar (Figure 3.10.1). All modern projections appeared to over predict distribution on the East Coast of the SI, however predictions elsewhere were relatively consistent with known distribution maps. Models $2 \mathrm{~d} 11$ and $2 \mathrm{~d} 12$ had the lowest $\mathrm{OR}_{10}$ omission rates and similarly relatively low $A \cup C_{\text {DIFF }}$ values $\left(A \cup C_{\text {DIFF }}=0.027\right.$, $\left.\mathrm{OR}_{10}=0.091\right)$. These models also had the second-best discriminatory ability $\left(A \cup C_{\text {train }}=0.90, A \cup C_{\text {test }}=\right.$ $0.88)$. As both $2 \mathrm{~d} 11$ and $2 \mathrm{~d} 12$ had the exact same statistics, the less complex $2 \mathrm{~d} 11$ was selected as the base model. 

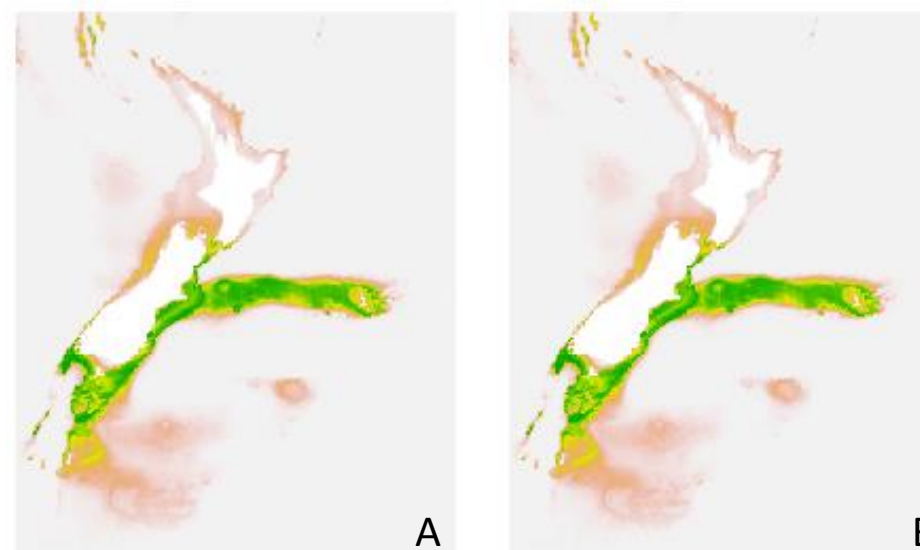

B
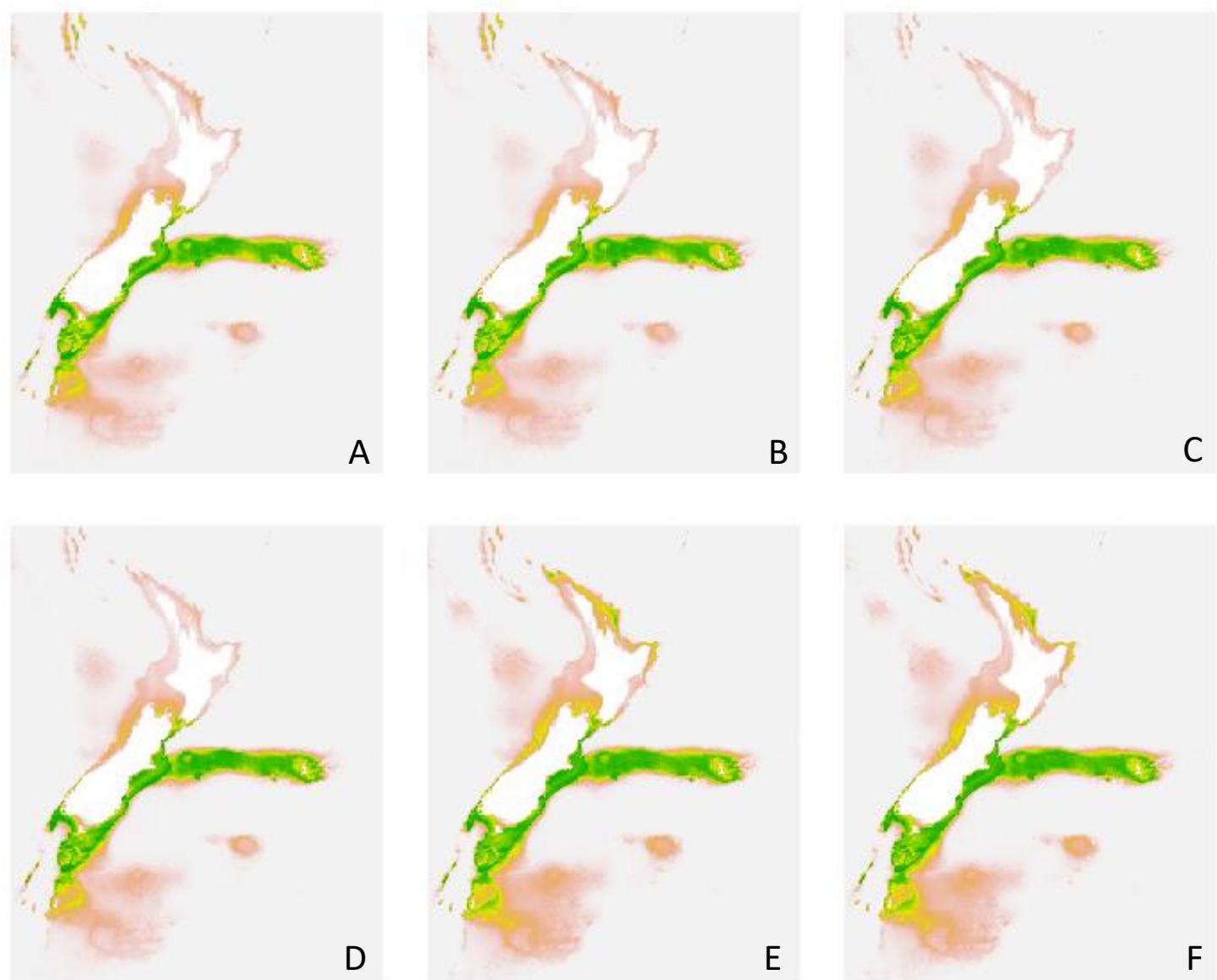

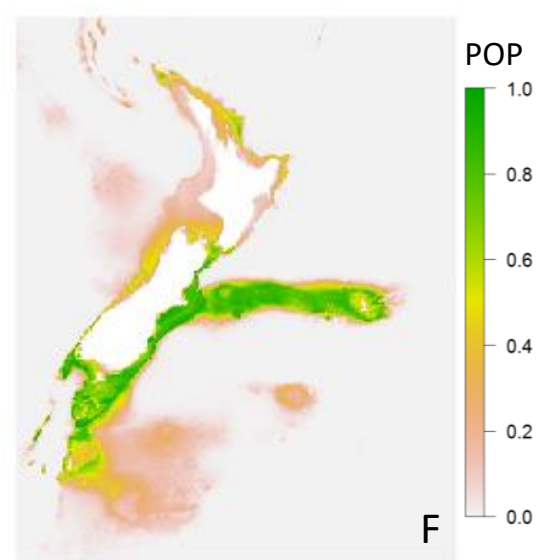

Figure 3.10.1: Arrow squid; Six best Maxent models projected on modern climate layers; $1 \mathrm{~d} 5$ (A), $1 \mathrm{~d} 6$ (B), $2 \mathrm{~d} 11$ (C), $2 \mathrm{~d} 12(\mathrm{D}), 3 \mathrm{c5}(\mathrm{E}), 3 \mathrm{c} 6(\mathrm{~F})$. Colours reflect probability of presence estimates between $0-1$. Darker green indicates higher POP and red the reverse. 


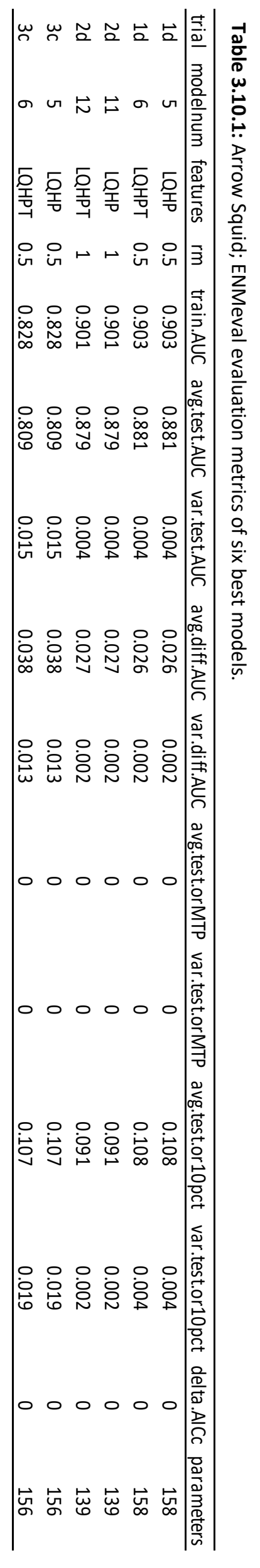




\section{Model Projections}

The 2050 projections looked relatively similar to the modern predictions, but varied more as climate scenarios presented more different conditions (Figure 3.10.2B-E). The predictions showed slight reduction of POP of arrow squid on the Chatham Rise, while a slight increase in POP was observed in the southern most extents of the distribution. This effect was most pronounced in RCPS 8.5. The effects of future climates on arrow squid POP thresholds were more cryptic than for other species, with increases in high POP in RCPS 25 and 4.5, while RCPS 6.0 and 8.5 generally showed decreases at in area with high POP (POP bins above 0.6) (Table 3.10.2). POP at low thresholds similarly increased slightly for all predictions in 2050. Overall changes in POP of arrow squid was relatively small for all RCPS in 2050.

2050 MESS maps suggested low levels of relative uncertainty in all RCPS (Appendix D.10). Highest certainty was consistently observed on the Chatham Rise and around the SI (Figure 3.10.3A).

\section{$\underline{2100 \text { Model Projections }}$}

The 2100 predictions seemed to follow the pattern of the 2050 predictions, with further increase in POP in southern regions around the Auckland Islands and western Campbell Plateau and decrease in POP along the Chatham Rise (Figure 3.10.2G-J). Again, this pattern was more pronounced in the RCPS 8.5, where Expansion onto the Campbell Plateau and withdrawal from the Chatham Rise was more substantial. The RCPS 2.6 prediction in 2100 was very similar to the RCPS 2.6 prediction in 2050, with similar POP values accompanying (Table 3.10.2). The remaining 2100 predictions were more varied. In RCPS 2.6, areas with high POP increased more than areas with low POP, while in RCPS 4.5, 6.0 and 8.5, areas with low POP increased more than areas with high POP, except for those that fell into the highest POP bin 0.9. In RCPS 8.5, areas with a POP of 0.2-0.3 increased almost as much as areas with a POP of 0.8-0.9. While large increases in the top POP bin (>0.9) was observed in all climate scenarios (35.42\%-69.60\%), the change in area assigned to other POP bins was more varied.

2100 MESS maps had slightly less certainty than the 2050 MESS maps (Appendix D.10). Uncertainty was most evident in RCPS 8.5 (Figure 3.10.3B). In this scenario regions northward of the mid $\mathrm{NI}$ showed the most relative uncertainty. 

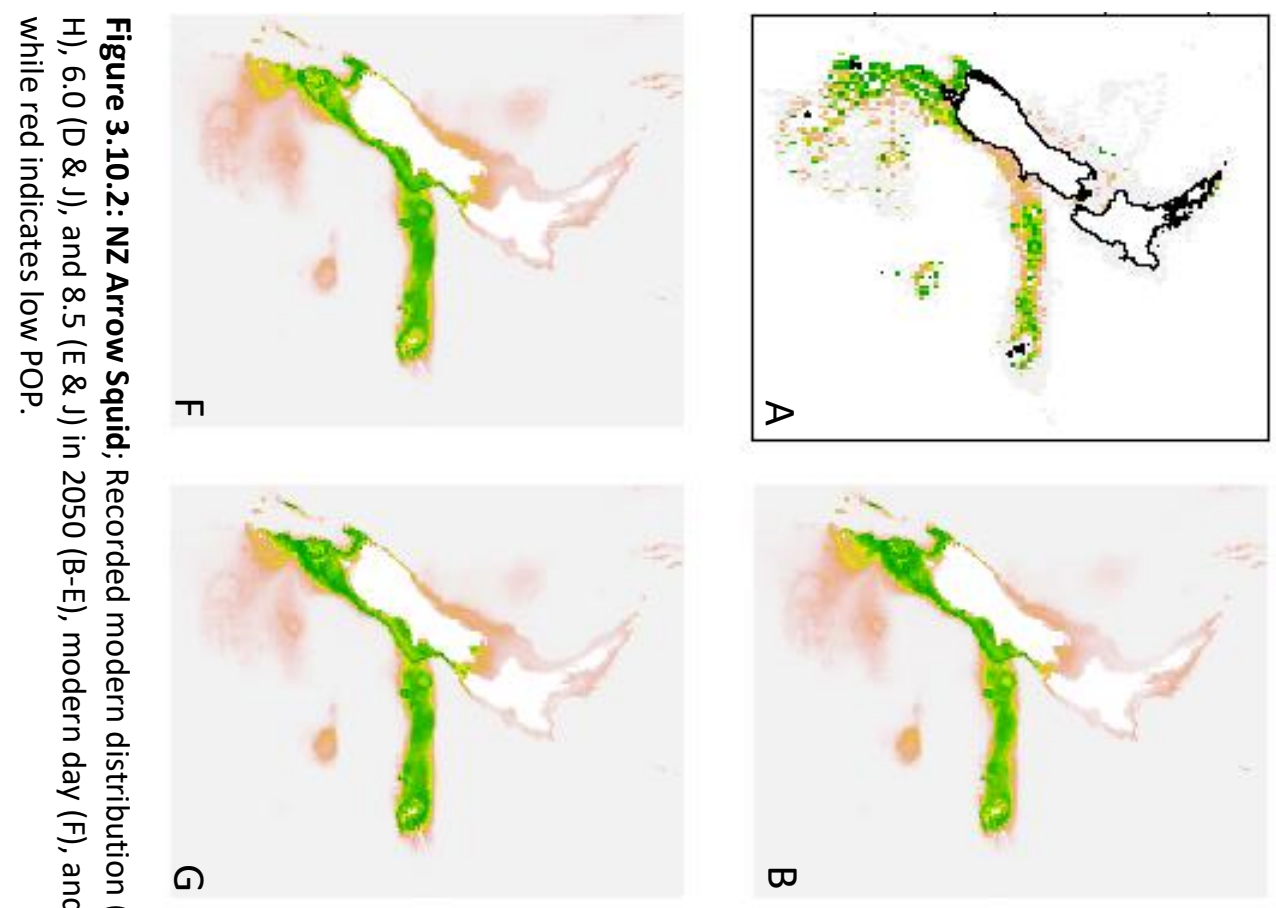

금

응 음

공

응 을

जे

勇

욱

윙 음

금 I

은.

蚛

吕

亦

ก ร

온옥

맹ㅁำ

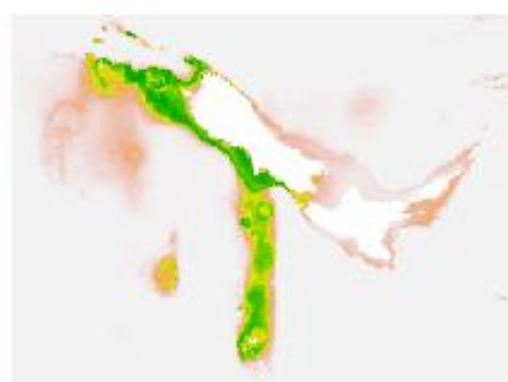

I

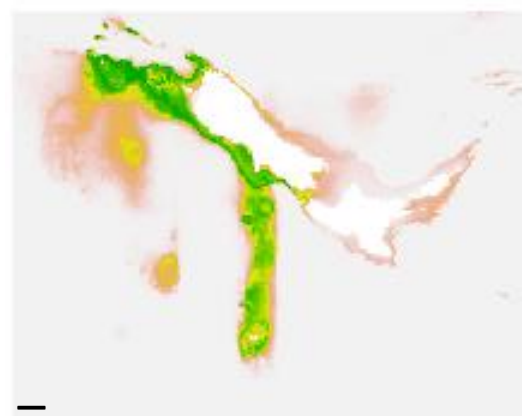

$\infty$
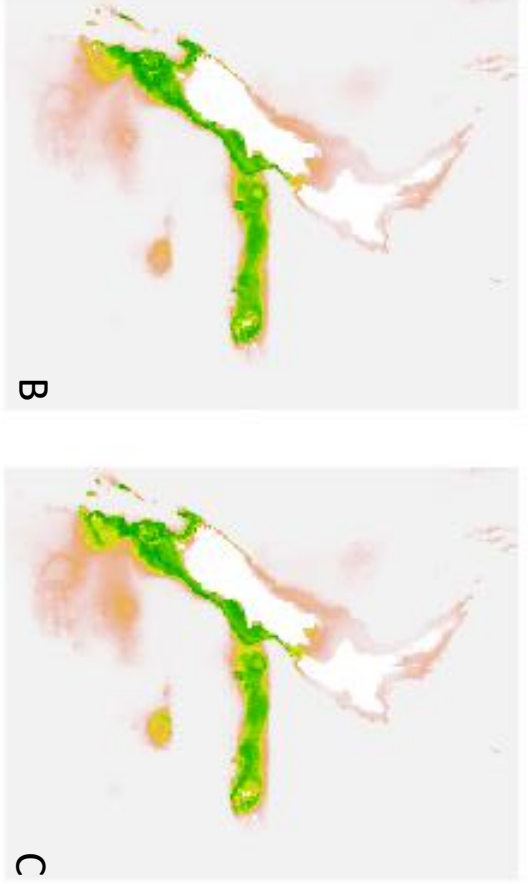

oo

ำ

竞.

吕

둥

o $\frac{1}{n}$
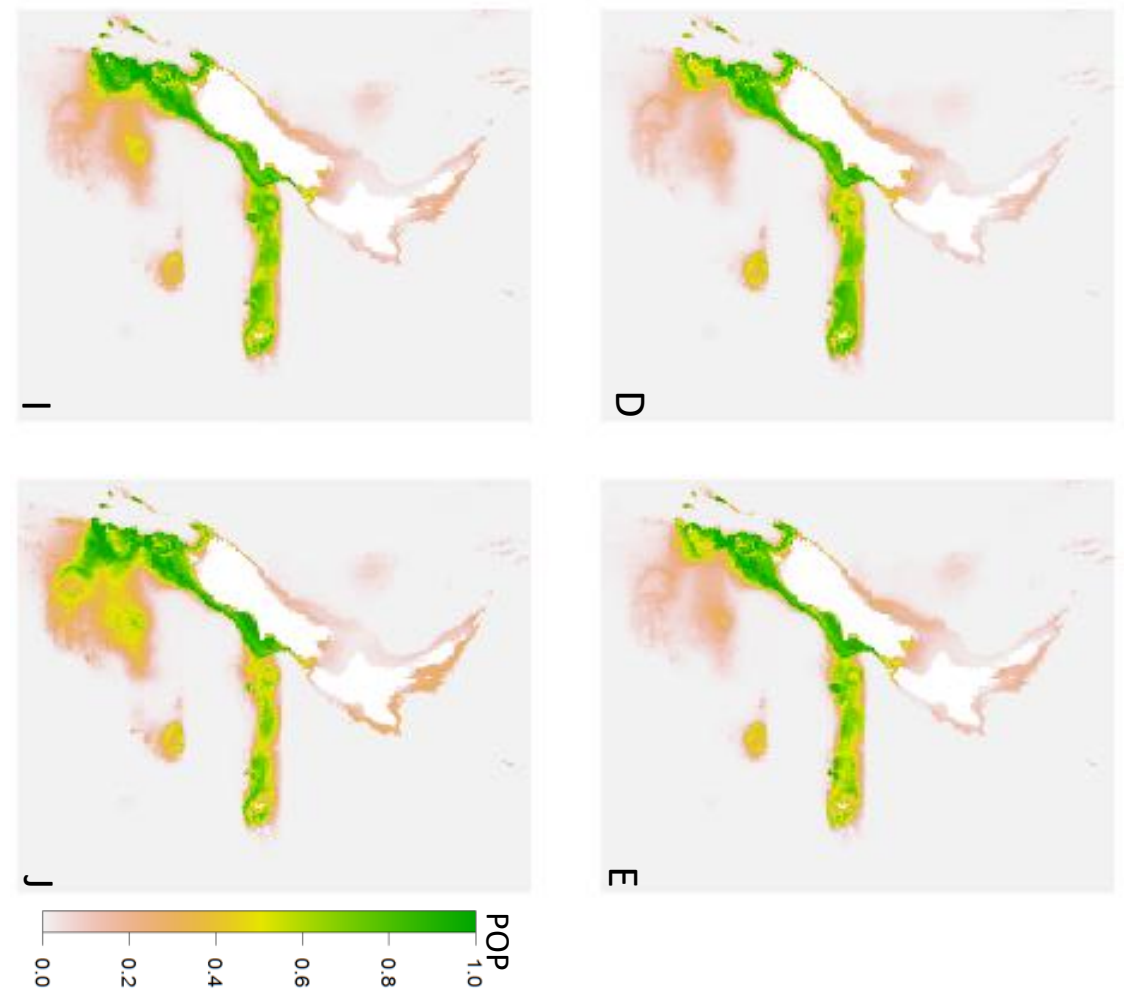
Table 3.10.2: Arrow squid; Change in POP values compared to the modern projection for each of the four climate scenarios 2050 and 2100.

\begin{tabular}{lllllllll}
\hline \multicolumn{7}{c}{$\mathbf{2 0 5 0}$} & \multicolumn{5}{c}{$\mathbf{2 1 0 0}$} \\
POP & RCP2.6 & RCP4.5 & RCP6.0 & RCP8.5 & RCP2.6 & RCP4.5 & RCP6.0 & RCP8.5 \\
\hline $\mathbf{0 . 9}$ & 41.298 & 4.471 & -11.308 & 14.029 & 41.719 & 35.416 & 49.786 & 69.602 \\
$\mathbf{0 . 8}$ & 8.378 & 3.351 & -14.841 & -17.36 & 17.162 & -7.069 & -1.348 & 0.084 \\
$\mathbf{0 . 7}$ & 12.198 & 3.879 & -10.045 & -23.657 & 16.656 & -9.793 & 1.971 & -8.978 \\
$\mathbf{0 . 6}$ & 9.133 & 4.462 & -4.676 & -12.08 & 12.868 & -5.549 & 7.661 & 0.45 \\
$\mathbf{0 . 5}$ & 6.862 & 8.351 & 1.504 & 2.471 & 8.694 & 1.169 & 12.384 & 18.394 \\
$\mathbf{0 . 4}$ & 5.887 & 9.568 & 5.815 & 7.488 & 5.496 & 4.564 & 16.441 & 44.029 \\
$\mathbf{0 . 3}$ & 1.403 & 6.424 & 3.983 & 4.135 & 2.056 & 1.787 & 21.736 & 53.8 \\
$\mathbf{0 . 2}$ & 1.303 & 9.185 & 4.899 & 5.962 & 3.296 & 2.425 & 26.148 & 59.026 \\
$\mathbf{0 . 1}$ & 0.92 & 10.14 & 7.432 & 7.591 & -6.07 & 1.585 & 19.516 & 31.031 \\
\hline
\end{tabular}
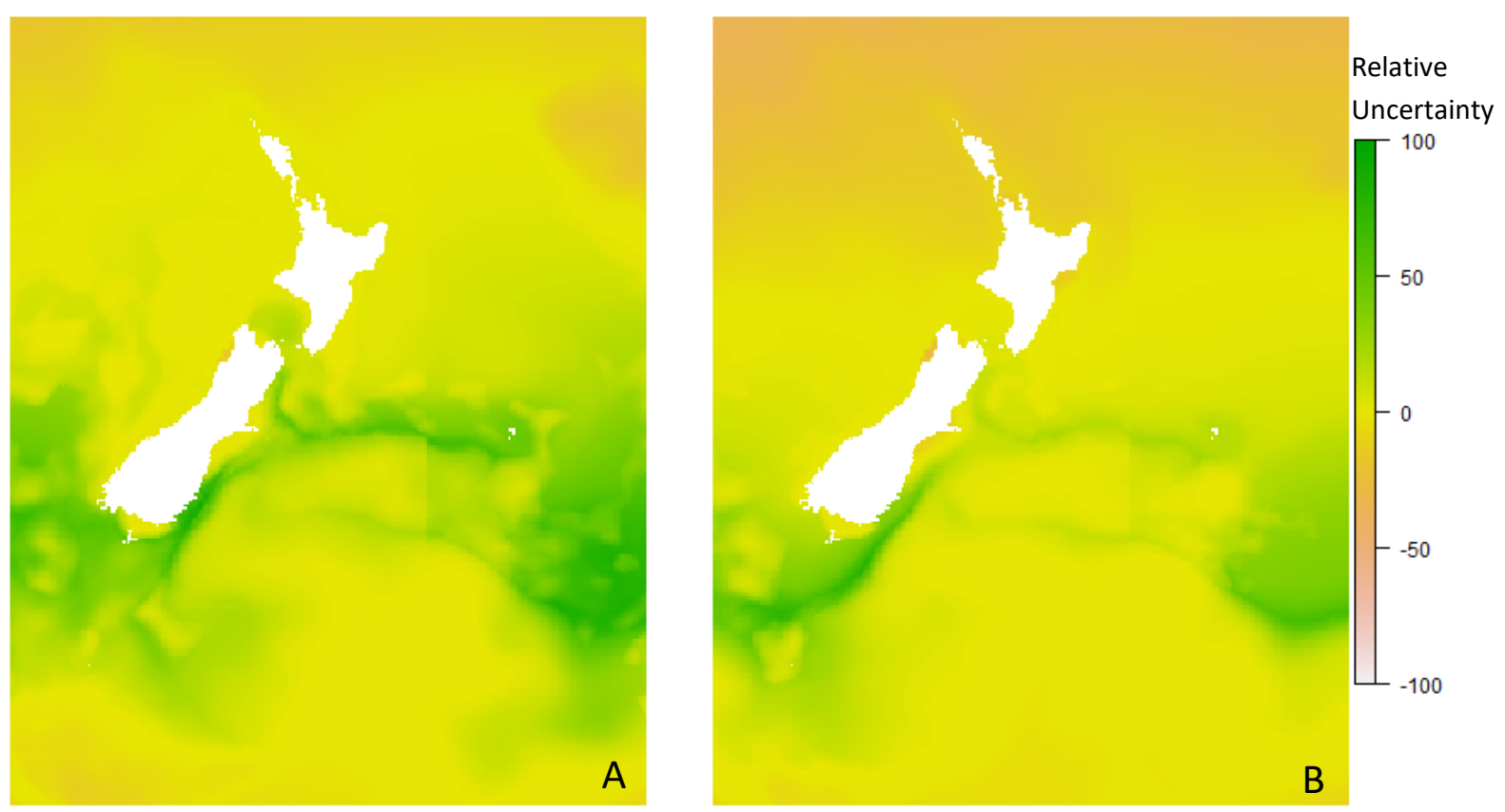

Figure 3.10.3: Arrow squid; MESS Maps of RCPS 2.62050 (A) and RCPS 8.52100 (B). MESS maps for all climate scenarios can be found in Appendix D.1. Negative sites indicate most relative uncertainty and positive sites indicate most relative certainty. 


\section{$\underline{\text { Variable Influence }}$}

The base model predicted that arrow squid were essentially absent below $-1000 \mathrm{~m}$ and had increased POP with mean ST mean until approximately $11^{\circ} \mathrm{C}$, where the response was fixed (Figure 3.10.4). SS mean was most preferable around 34psu and response decreased above and below that. SS range had a negative relationship with POP, although did not contribute to the base model. ST range had a cryptic effect with limited model contribution. ST mean contributed more to the base model than other climatic variables (Figure 3.10.5).

In the contemporary climate ST mean was the main limiting climatic variable in southern regions, particularly around the Campbell Plateau, while SS mean was the main limiting variable around the $\mathrm{NI}$ and western New Zealand (Figure 3.10.6A). In the future limiting factor plot, ST mean remained the main limiting factor but in a much smaller region on the southern half of the Campbell Plateau (Figure 3.10.6B). SS mean remained the main limiting factor in northern regions and became more limiting on the Chatham Rise and upper Campbell Plateau. The area where SS range was a limiting factor also increased in the future climate, particularly on the Chatham Rise.
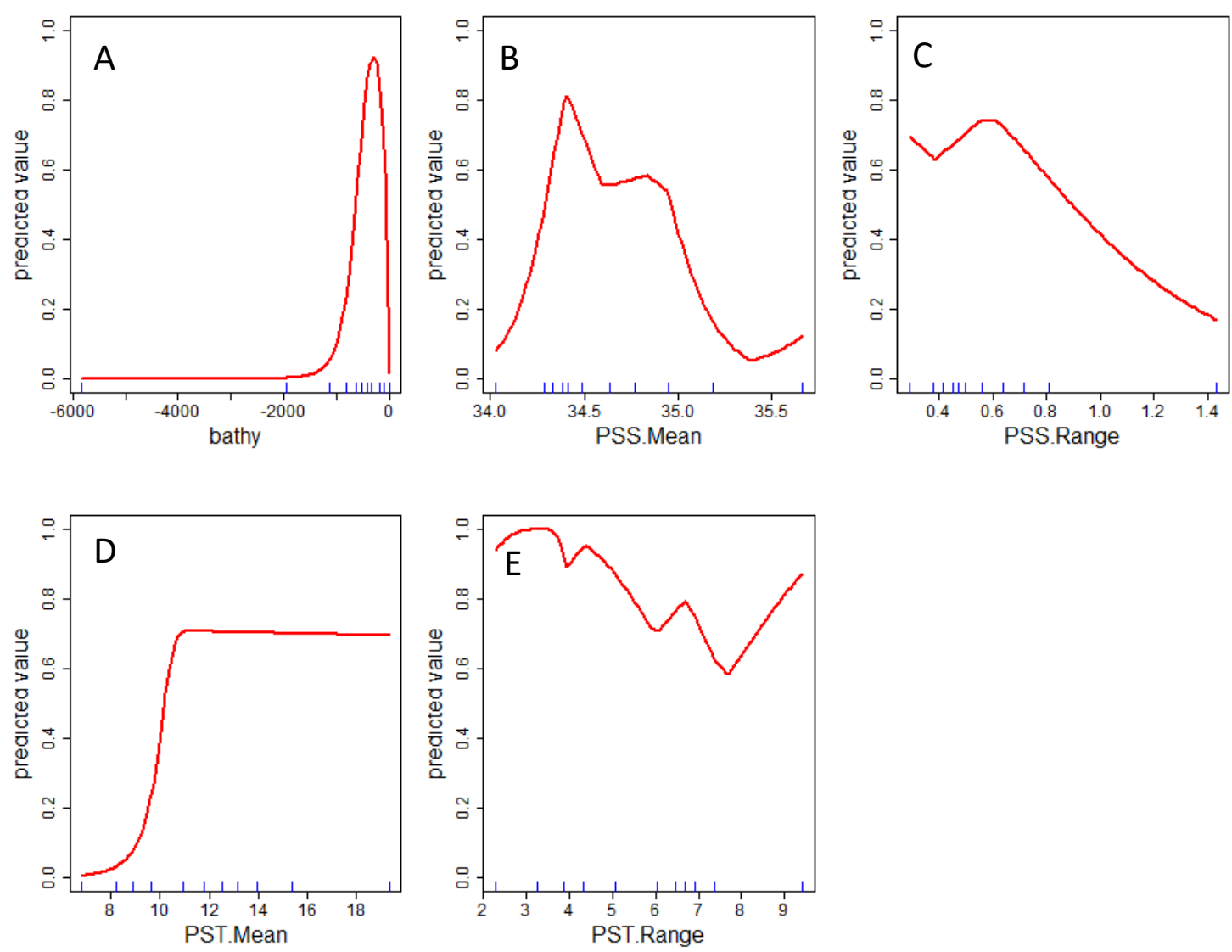

Figure 3.10.4: Arrow squid; Predictor response curves indicating how variables used for training affected the base model. Model predictions (red lines) and observations (blue data rug of deciles) are shown. The variables are bathymetry $(A)$, surface salinity mean $(B)$, surface salinity range $(C)$, surface temperature mean $(D)$, and surface temperature range $(E)$. 


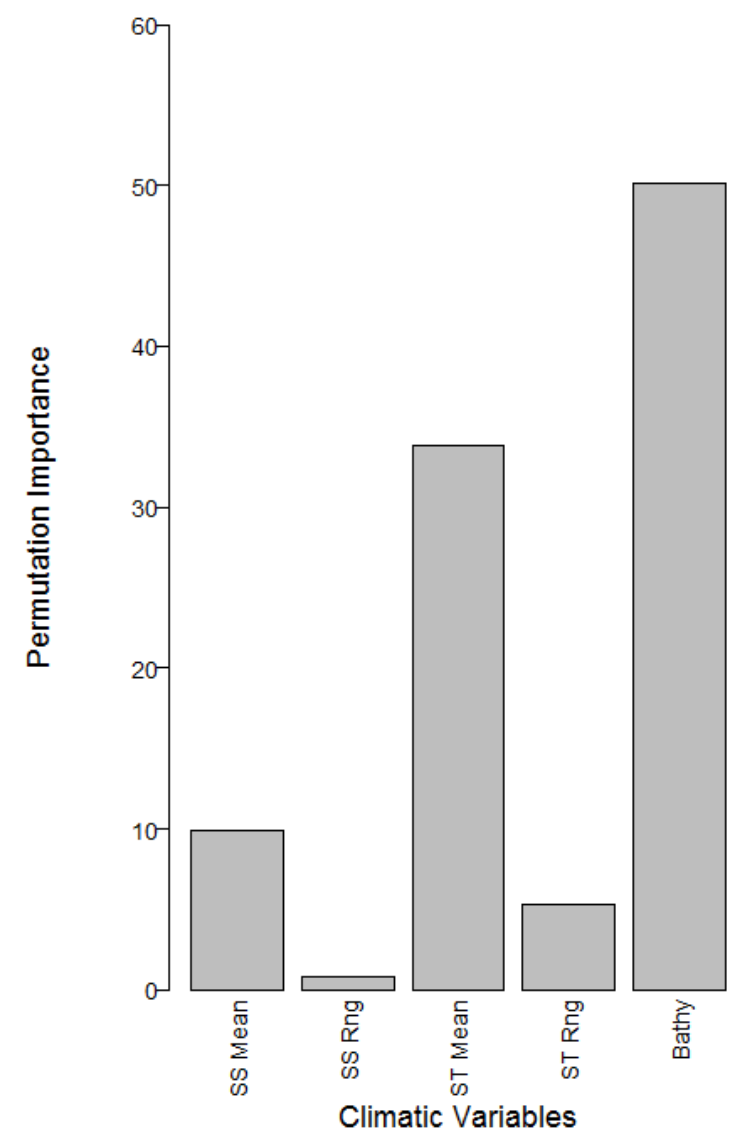

Figure 3.10.5: Arrow squid; Predictor variable contribution based on permutation importance (Phillips, 2006). Variable names are shortened here to fit: SS = Surface salinity, ST = Surface temperature, Rng = range, Bathy = bathymetry.
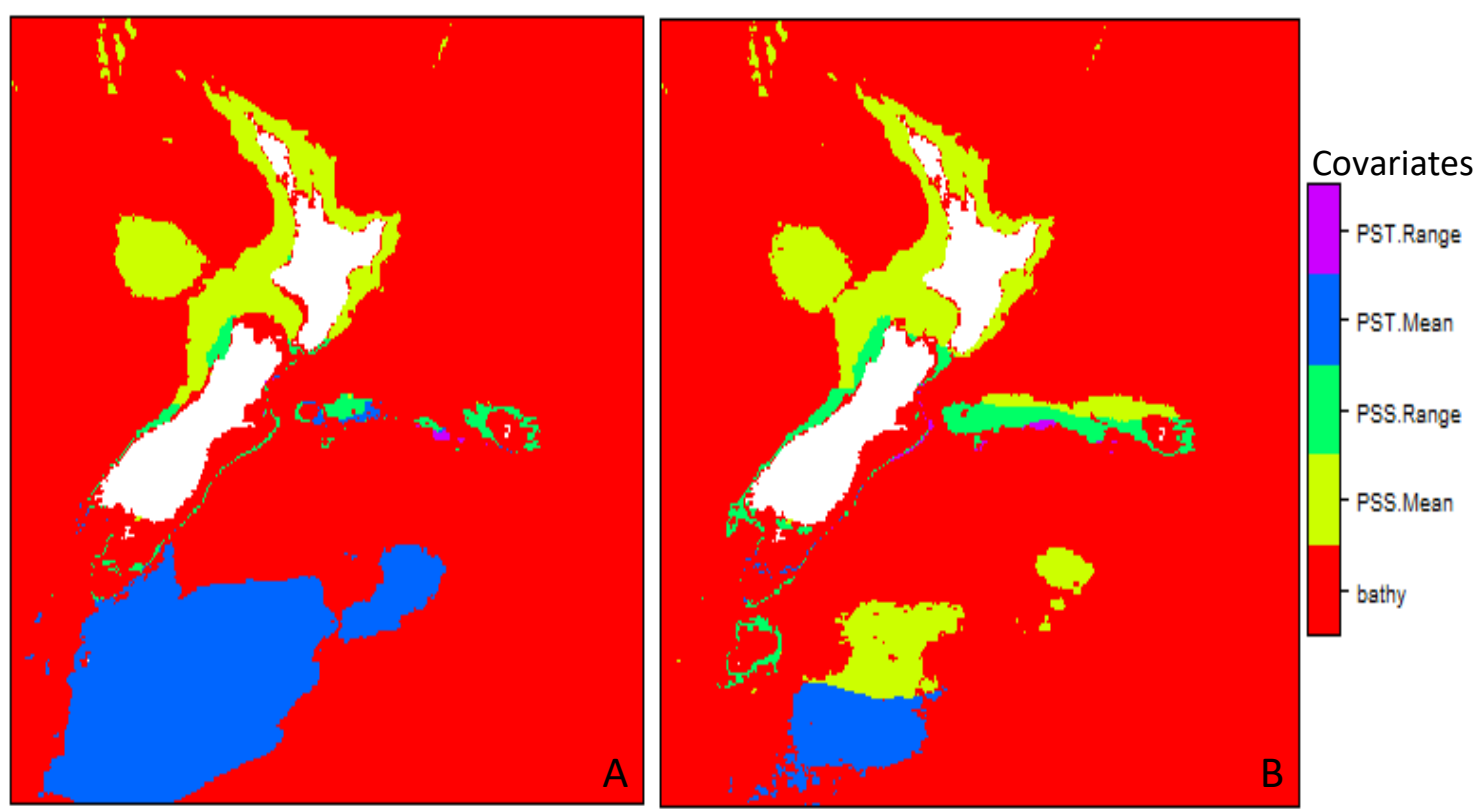

Figure 3.10.6: Arrow squid; Limiting factors plots of Modern (A), and RCPS 8.5 in 2100 (A) climates. Colours indicate the most limiting variable to distribution in that area. 


\subsection{1: Results Summary}

\section{Model Selection}

Criteria for base model selection required some flexibility, mostly owing to variation in $\mathrm{OR}_{10}$ values between species. For each species the six 'best' models were chosen with the lowest $\mathrm{OR}_{10}$ values possible. The highest this criterion was set was $<0.17$ for hoki models, and the lowest was $<0.11$ for trevally models, suggesting these were the datasets that produced the most and least overfitting respectively. Other selection criteria did not require adjustment.

Overall, base models were good at representing the preferred climatic niches of the species studied. This was demonstrated by how well the models projected on the modern climates matched their known distributions. Some distributions were slightly over or under predicted but usually represented ranges well. For some species, such as orange roughy and there was substantial variation between the selected best models. Those with substantial variation between base models should be interpreted with cation, as future projections varied with base model choice where this was the case. Usually however, all six best models looked similar and had comparable variable contributions, response curves, MESS maps and future projections.

\section{Future Projections}

Differences from modern predictions were usually more extensive in 2100 predictions than those under the same RCPS in 2050. There was more variation between climate scenarios in the 2100 predictions than 2050 predictions, which frequently looked similar regardless of the different RCPS. RCPS 8.5 typically experienced more substantial changes than all other climate scenarios.

Southward extensions or shifts were observed in all future predictions of most species (snapper, john dory, trevally, tarakihi, arrow squid, orange roughy and scampi). For trevally, tarakihi and arrow squid southern extensions coincided with contractions from northern regions. In snapper, john dory, orange roughy and scampi predictions, this northern contraction was not observed. Instead, POP increased in northern regions, which remained stable areas of high POP in all RCPS for these four species. Both snapper and john dory had exceptionally similar responses to each RCPS of comparable scale, particularly evident in their expansions down the West Coast of the South Island. The shifts or expansions southward were more extensive in climate scenarios that were most different from the contemporary climate. The only inshore species that did not experience overall increased POP in future predictions was trevally. 
Future projections for hoki, ling and southern blue whiting, all showed considerable loss of suitable conditions, particularly on the Campbell plateau and Chatham Rise. This loss was more substantial in climate scenarios that deviated most from contemporary conditions. The ling and hoki RCPS 8.5 projections in 2100 showed strikingly similar contractions to the southern half of the Chatham Rise. Orange roughy was the only deep-water species that experienced increased suitable conditions in all future scenarios, the most substantial increase being in RCPS 8.5 in 2100.

\section{Probability of Presence Changes}

Some changes in POP bins were very small, so $10 \%$ change was established as the point at which change observed was considered noise instead of true change. This was assigned arbitrarily to aid interpretation and is shown as a dashed line in Figure 3.11.1. Any POP changes that were less than $10 \%$ increase or decrease were interpreted as having no change.

The highest quality habitat was usually affected the most. For species that consistently experienced decline, such as hoki, ling, southern blue whiting, and trevally, the area with the highest POP frequently declined the most, while areas with average POP declined much less (Figure 3.11.1). In some scenarios this coincided with an increase in areas with low POP, while in others, the lowest quality habitat also decreased but to a lesser extent than the higher quality habitats. For ling, this pattern was only observed in the RCPS 8.52100 prediction.

For species that experienced increased suitable conditions, such as john dory, snapper, tarakihi, and orange roughy, areas with high POP increased more than areas with lower POP. For scampi this was not true in RCPS 26 and 4.5 in 2100 predictions. In john dory, snapper and tarakihi projections, the increase in high POP areas coincided with a decrease in low POP areas. The general pattern of change was frequently amplified by extreme loss or gain of high-quality habitats compared with areas with low quality conditions.

\section{Uncertainty}

Consistently the MESS maps showed most relative uncertainty was in northern regions. This effect was most pronounced in RCPS 8.52100 for all species. Uncertainty was more varied across each RCPS in the 2100 MESS maps than the 2050 MESS maps, which often all looked similar, regardless of the RCPS. 2100 mess maps usually had less certainty than 2050 MESS maps.

Of the inshore species, tarakihi seemed to have the most certainty in predictions overall, as MESS maps rarely displayed large areas of uncertainty. Trevally showed high relative uncertainty in 
furthermost southern regions, as well as the substantial northern uncertainty estimates observed in all inshore species base models. One deep-water species, scampi, also displayed this pattern.

Of the deep-water species, hoki and ling showed very little uncertainty in all climate scenarios, except for RCPS 8.5 in 2100 in a narrow area around the northernmost extent of the study region. The orange roughy MESS maps had slightly more uncertainty, particularly in the southernmost and northernmost edges of the study area and noticeably more so in the RCPS 8.52100 prediction. The southern blue whiting MESS maps showed more relative uncertainty in the northern regions than all other species, which extended to the bottom or the NI or halfway down the SI in some scenarios. Conditions in future climates were outside of the training range of the data used to train southern blue whiting models in a large proportion of the study region.

\section{Variable influence}

Surface temperature (ST) mean consistently contributed more to base models than any other climatic variable. The only exception was the southern blue whiting base model, in which Surface salinity (SS) mean contributed the most. In particular, ST mean contributed considerably to john dory, snapper and arrow squid ( $>30 \%$ permutation importance) base models. For john dory and snapper all other climate variables almost entirely did not contribute. SS mean contributed notably, although less so than ST mean, to tarakihi, scampi, arrow squid and hoki base models. For these four species the permutation importance of SS mean was approximately $10 \%$, which was almost half that of ST mean for scampi and hoki. Except for hoki, all species had decreased POP with high SS mean. For ling, trevally and orange roughy even the highest contributing variable ST mean, did not contribute more than approximately $10 \%$ to the base models. SS and ST range consistently contributed very little or not at all.

For snapper, john dory and orange roughy, the most preferable ST mean was the highest experienced during model training, so response was fixed at this temperature. Mean temperatures experienced higher than this in future climates, were assigned the same response. This was similarly observed in scampi, however more of its mean temperature range was experienced during training. While mean temperature preference peaked just below the highest temperature experienced during training, the temperature response for scampi was still fixed as highly preferable for all higher temperatures in future climates. The temperature response was the opposite in hoki, ling, southern blue whiting, trevally, and tarakihi, where the highest temperature experienced during model training was assigned a very small or 0 value, so response to higher temperatures was fixed as relatively less preferable. 
Some response curves showed responses to variables that were difficult to interpret. This was particularly an issue when the response to ST mean was cryptic because it usually contributed most to the models. For example, hoki and ling had implausible ST mean responses, with large sudden increases in predicted values at low ends of their ST mean range. In both cases this coincided with large increases at the upper end of their SS mean range.

In contemporary climates ST mean was the most distribution limiting variable in the southern range of snapper, john dory, trevally, tarakihi, scampi, arrow squid and orange roughy distributions, while other variables were limiting distribution in northern regions. In the future climates, the extent to which ST mean was a limiting factor was reduced and shifted southwards in most cases, expect for trevally. For trevally the future limiting factor plot showed ST mean limited distribution in northern regions instead. In contemporary climates hoki and ling distribution was most limited by ST mean in most regions around New Zealand, but less so in southern regions in future climates where SS mean became more limiting to their distribution. For southern blue whiting, SS mean limited distribution most throughout the study region in both contemporary and future climates. 


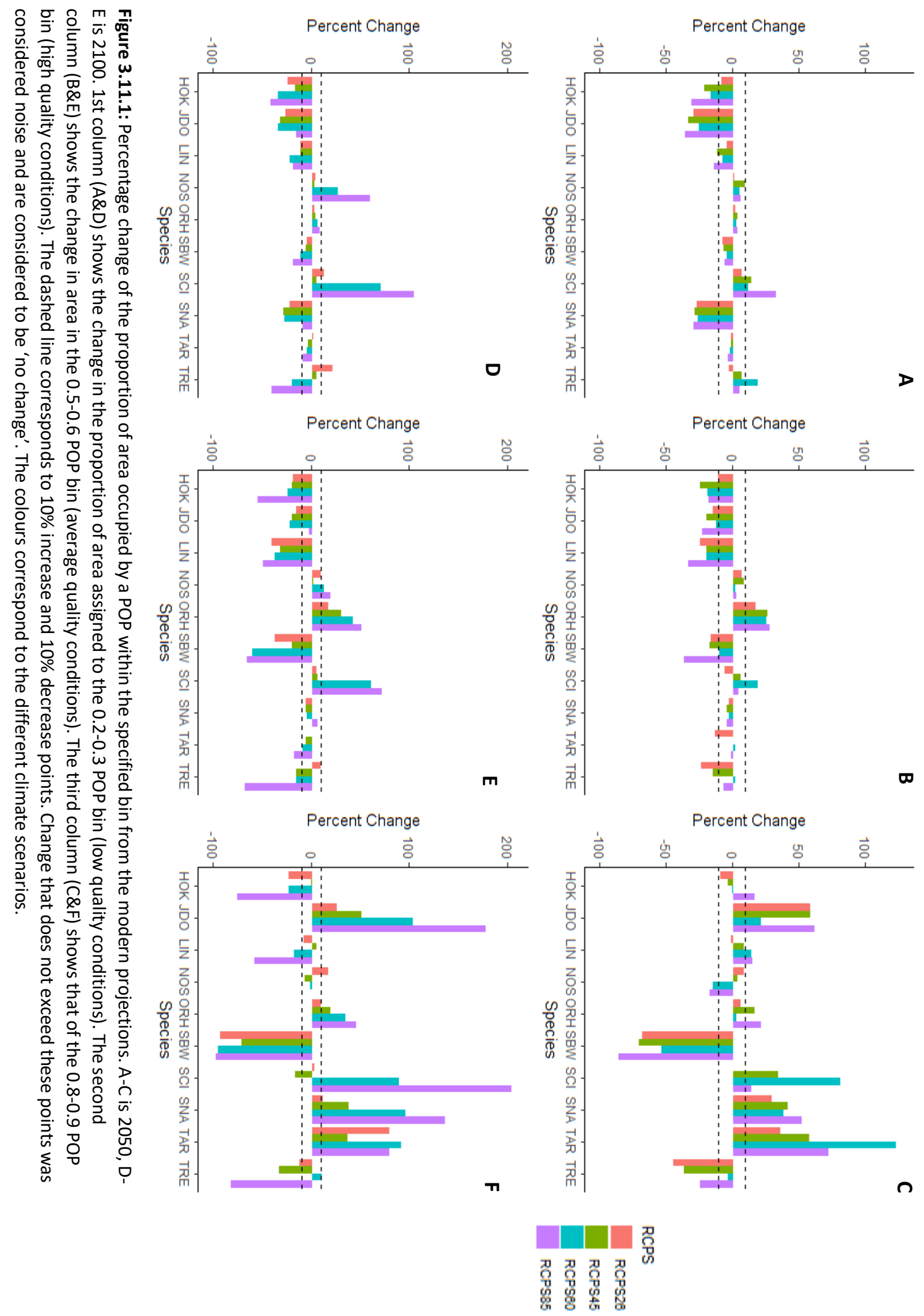




\section{Chapter 4: Discussion}

\section{1: General Summary}

This study has provided an idea of how changes in annual mean temperature may influence many marine species distributions under future climate change in New Zealand. Bathymetry and mean temperature consistently influenced and limited projected distributions the most. The southward expansions of fish distributions were often associated with the reduced effect of mean temperature as a limiting factor in southern regions. Furthermore, species that experienced shifts from northern regions southward due to reduced effect of mean temperature limiting distribution in southern regions, simultaneously experienced increased influence of mean temperature or salinity as a limiting factor in the north. Other studies that considered a wider range of variables also often found mean temperature to be a main influencer of species distributions (Alabia et al., 2015; Derville et al., 2018; Torres et al., 2013). Although less so, mean salinity also had a notable contribution to some species models. The relevance of this is difficult to discern, due to salinity mean and mean temperature being highly correlated (Appendix B).

Predictions suggested that different species will have varied responses to future climates. All species studied here were predicted to experience changes to their respective areas of suitable habitat that were consistently most extreme under the RCP 8.5 climate scenario. Changes occurred as net loss or gain of area of suitable habitat and/or shifts in the geographical distribution of areas of suitable habitat. Distribution shifts varied in both direction and extent.

Directional poleward shifts along a latitudinal gradient were observed across most species, while others contracted inwards towards the centre of their ranges. Usually, species that showed directional shifts were those typically found in sub-tropical or temperate waters, while those in subpolar regions declined but had no obvious directional shift. In this study, snapper, john dory, trevally, tarakihi, orange roughy, scampi, and arrow squid were frequently predicted to have poleward distribution shifts. These results were consistent with numerous studies that have predicted marine species in temperate or sub-tropical regions will exhibit poleward movement in response to warming climates (Alabia et al., 2015; Barange et al., 2016; Bruge et al., 2016; Christian \& Holmes, 2016). In large scale global studies, this response has been produced across a diverse range of marine species in many different regions around the world (Cheung et al., 2009; Morley et al., 2018; Robinson et al., 2015). However, this was the first study to predict this trend specifically in New Zealand, and local areas that may be most affected were therefore highlighted. 
The response of sub-polar and polar species is more uncertain. Cheung et al. (2009) predicted local extinctions of sub-polar marine species, while Morley et al. (2018) predicted continued poleward movement along coastlines in the northern hemisphere. For marine species in sub-antarctic New Zealand, there is no contiguous coastline to continue following poleward. This could inhibit their ability to track cooler waters southward. Movement may be constrained by the relatively narrow continental shelf around New Zealand as water further south may be too deep, in the absence of a depth range shift response. Sub-antartic species in New Zealand may therefore be more vulnerable than those in more temperate regions. This is reflected by hoki, ling and southern blue whiting results in this study, none of which exhibited clear poleward shifts but instead had temperature responses that were limited by bathymetry. Each of these species experienced contractions from the Campbell Plateau, the southernmost part of their ranges and overall reduction in the area of suitable habitat available in all climate scenarios. Hoki and ling projections showed contractions from both northern and southern sides of their ranges, resulting in decline to narrow areas along the southern Chatham rise and East Coast of the South Island. Southern blue whiting suitable habitat was lost more than that of any other species and this loss did not occur in a clearly directional way. The predicted declines of species in sub-polar regions may be amplified by intensive invasion by temperate species (Cheung et al., 2009).

Predicted southward shifts, did not necessarily coincide with stable total area of suitable habitat. There was variation in whether poleward shifts involved gain, loss or no change in the amount of suitable habitat. Species that shifted poleward were usually predicted to have increased overall probability of presence, except for trevally and arrow squid. Several species, including snapper, john dory, scampi and orange roughy had projections that showed the areas these species already occupied, and the areas immediately surrounding them, would become more favourable as climates changed. For john dory, snapper, and scampi the increase in highly suitable habitat also coincided with their expansion southward to regions they have previously not been reported. John dory and snapper expanded to the very bottom of the West Coast of the South Island and Stewart Island, while scampi expanded onto the Campbell Plateau. For projections that showed range expansion, but not contraction, overall probability of presence increased substantially, because it increased both in regions already occupied by the species and in areas that they expanded into (scampi, snapper, john dory, Orange Roughly).

Tarakihi, arrow squid, and trevally also showed poleward movement, but this coincided with contractions in the northern regions of their range. For tarakihi and trevally this involved almost complete removal from the North Island by 2100 in climate scenarios 6.0 and 8.5. Arrow squid 
expanded onto the Campbell to areas much further than its range currently extends but also lost suitable habitat on the Chatham Rise. The range shifts of each of these species was associated with varied changes to the overall amount of suitable habitat. Trevally distribution shifted and contracted, as it experienced net loss of suitable habitat, while tarakihi shifted but experienced a net increase in suitable conditions in all climate scenarios. Arrow squid range shifted but there was usually little to no change to the amount of suitable habitat, even in the most extreme scenario.

\section{2: Uncertainty in projections}

\subsection{1: Incomplete and implausible temperature responses}

The interpretations of the results produced in this study rely on the assumption that the models somewhat accurately represented species responses to the climate variables. Response to mean temperature was more important than that any other climate variable for most species so this discussion will be focussed on that aspect. Incomplete mean temperature response curves likely demonstrated an inability to capture the entire thermal tolerance range. The species where the upper range limits of thermal tolerance were not fully captured were snapper, john dory, orange roughy, arrow squid and scampi. Implausible response curves could demonstrate unreliable capturing of response in some areas. Additionally, response curves for hoki and ling were somewhat implausible at low temperatures.

Base models failed to capture the bottom temperature range of hoki and ling and there was a sudden increase in predicted value in response to temperatures lower than $\sim 10^{\circ} \mathrm{C}$. This seemingly implausible temperature response could be a result of lack of records in areas associated with these lower temperatures. This may have been due to exclusion of data from areas far enough southward to capture the edge effects of their distributions. Inclusion of these data could have resolved tail ends of temperature response in a more plausible way, with declines in lower temperatures eventually becoming apparent, rather than sudden increases. The thermal tolerance range for hoki and ling is therefore likely narrower than that represented by the models. The lack of data at lower end of the thermal tolerance likely would not substantially influence future model projections as lower values than that experienced in model training were not present in future climates. However, if these models were projected onto cooler climates, (such as in a New Zealand paleoclimate projection), this sudden increase in response to cooler temperatures would raise issues, as the response to lower temperatures would be fixed at their high predicted value.

For snapper, john dory, and orange roughy, the base models for these species were trained without information on the upper bound of their thermal tolerance. Lack of occurrence records associated 
with higher temperatures was not necessarily due to an inability to tolerate higher temperatures but did result in their response to higher temperatures not being fully captured by the base models. No occurrence records were associated with mean temperatures higher than approximately $19^{\circ} \mathrm{C}$ for snapper and john dory, and $20^{\circ} \mathrm{C}$ for orange roughy. The maximum mean surface temperatures within the study region did not surpasses $20^{\circ} \mathrm{C}$ in modern climate layers. Therefore the highest temperature available was assigned the most preferable and no information about how these species respond to higher temperatures was available to the base models. Maxent fixed their response at around $19-20^{\circ} \mathrm{C}$ so that the response to mean temperatures higher than this in future climates was assumed to be the same, regardless of how warm it gets. Higher temperatures were present in the future climate scenarios, particularly in northern regions, but their response to these higher temperatures is fixed at the same response to the highest temperature used in model training. This explains why the probability of presence for these species appeared to increase greatly in northern areas in most future projections. Without information on the upper bounds of their thermal tolerance, it is not certain whether the warmer conditions in future climates would be tolerable for these species. If they are not, a contraction from northern regions in conjunction with a southern expansion might be expected, as was observed for tarakihi, a species with more of its mean temperature tolerance range included its base model. Tarakihi showed varied contraction from northern regions in all climate scenarios by 2050.

In order to capture the full response of most sub-tropical or temperate species in New Zealand to changes in temperature, incorporation of occurrence records from regions where mean temperature is higher would be useful. Records from areas further north of New Zealand such as Northfolk or Kermadec Islands or from the Australian region west of New Zealand may achieve this. Information from fish with distributions that extend further north along coastal Australia could provide useful information about the upper limits of their thermal tolerance and how tolerance tails off along a contiguous coast. If their response declines past a certain temperature threshold around Australian coasts, this would be important information to include in these models. A restricted range correlated with warmer temperatures would be important information to include in niche descriptions for a species.

Without information from other regions of the species ranges, comparing projections with available biological information may be a limited but still useful approach. Snapper larvae size and abundance increases as water temperature rises up to $24^{\circ} \mathrm{C}$ (Cassie, 2005). Previous studies have shown that there is very little difference between snapper survival in $15^{\circ} \mathrm{C}$ versus $24^{\circ} \mathrm{C}$ waters however it is known that mortality occurs above $27^{\circ} \mathrm{C}$ (Fielder et al., 2005). In even the most extreme climate 
scenarios, mean temperature is not expected to exceed $23^{\circ} \mathrm{C}$ within the study region (Appendix C.2D). John dory abundance also increases with mean temperature, and they have been reported in areas that have experienced temperatures higher than $22^{\circ} \mathrm{C}$ in the Mediterranean Sea (Maravelias et al., 2007). Thus, increased snapper and john dory POP in northern New Zealand in climate scenarios where this region becomes warmer, seems to be a reasonably well supported prediction.

It is harder to determine how surface temperature will influence deep-water species like orange roughy. The temperature in deep water is typically colder than at the ocean surface. Orange roughy are usually caught in water that is between $4.5-6.5^{\circ} \mathrm{C}$ but occasionally are found at temperatures up to $10.2-11.2^{\circ} \mathrm{C}$ (Branch, 2001). Deepwater temperature in regions around northern New Zealand are expected to be about $5^{\circ} \mathrm{C}$ on average in the most extreme scenario (RCPS 8.52100 ), and only exceed $10^{\circ} \mathrm{C}$ inshore and in shallow areas within the study region (Assis et al., 2018). Based on the information available for the situation, the orange roughy projections appear to be reasonably well supported.

The arrow squid base model also showed incomplete response to warmer mean temperatures , likely because data informing the upper bound of their thermal tolerance was not included in model training. The temperature response of arrow squid appeared unusual as it was fixed as highly preferable for all temperatures above $11-12^{\circ} \mathrm{C}$. In New Zealand there are two species of arrow squid, N. sloanii and N. gouldi (McCoy 1888). It has been notoriously difficult to differentiate between these two species, particularly with females (Dunn, 2009; Uozumi, 1998). Despite this, their geographic distributions in New Zealand are clearly separate. N. gouldi is dominant around the North Island, top of the South Island and West Coast of the South Island. N. sloanii is dominant on the Chatham Rise, the East Coast of the South Island, and is exclusively found on the Campbell Plateau and Bounty Platform (Uozumi, 1998). It is often assumed that arrow squid found on the Chatham Rise are N. sloanii but N. gouldi exist here too, albeit in lower numbers (Uozumi, 1998). It is likely that some $N$. gouldi squid have been misidentified as $N$. sloanii and consequently their temperature responses inadvertently added to the data set. The response shown by the base model may therefore reflect an aggregate response of the two arrow squid species. The fixed response to high temperatures could be a result of incorporated signal from the northern species $\mathrm{N}$. sloanii. If this was the case, thermal tolerance was likely overestimated and projected shifts or decline of suitable habitat in northern regions potentially underestimated. Furthermore, inclusion of $N$. gouldi records would likely have resulted in underestimated extents of poleward shifts of $N$. sloanii.

It is less clear why the model failed to predict the upper limits of the thermal tolerance for scampi. Scampi are endemic so thermal tolerance should have been captured by models. The inability to do 
so may have been be due to some other boundary associated with the Chatham rise, limiting their modern distributions so dispersal further north into warmer waters does not occur. Use of sea surface environmental layers to model benthic species such as scampi could also have reduced the ability of models to capture full thermal response or monitoring of scampi could be failing to capture their full geographic range. Either way, the information used to train the base model did not appear to resemble scampi habitat well and therefore detection of environmental signal in scampi was problematic.

\subsection{2: Novel climates due to variables with values outside of the training range}

MESS analysis allowed visualisation of where novel conditions caused uncertainty in predictions. The MESS maps showed that in some future climate scenarios, conditions were substantially different from conditions experienced during model training, particularly in northern regions. The southern regions often had relatively low uncertainty because the associated conditions were experienced in model training, albeit in different areas (usually further north). High uncertainty in northern regions was recurrent throughout predictions, particularly for the RCPS 8.5 predictions. This uncertainty and the relevance of it is discussed below. The uncertainty was likely caused primarily by the increased mean temperature in these northern regions. There were frequently temperatures in northern regions of future climates that most base models had not encountered during model training. Northern New Zealand was therefore difficult for most models to accurately predict, particularly for species with thermal tolerance fixed at high temperatures, or with training data that only occupied a narrow region.

How do novel climates affect prediction interpretations?

MESS maps indicated where projections should be interpreted cautiously, particularly species with fixed responses to high temperature; snapper, john dory, orange roughy, scampi and arrow squid. For these species, high uncertainty in northern regions indicated areas where temperature was likely to be higher than the temperature at which their response was fixed. Increased probability of presence in these areas associated with high uncertainty was a likely a direct result of this fixed response. The further from the fixed value the future climate was, the more uncertain the true probability of presence was in these areas. Therefore, areas that had high uncertainty and were also predicted to have high probability of presence values for any species, should be interpreted with caution (Carneiro et al., 2016; Elith et al., 2010). Large observed increases in probability of presence in northern regions for these species in future projections may not be realistic representation of responses to warmer climates. Without information on the upper thermal tolerance ranges of these 
species, the predicted probability of presence in the northernmost most part of their ranges remains uncertain.

\section{$\underline{\text { Uncertainty differences between species }}$}

The amount of uncertainty was a result of the range of conditions associated with the training data. Training data is the combined occurrence and background data used for model training. Given a small range of conditions were represented in the training data, projecting onto climates with a wider range of conditions or much higher temperatures would result in large areas of uncertainty. Where occurrences were widespread around New Zealand, there was less uncertainty in predictions, particularly if these occurrences incorporated regions of higher latitudes, such as tarakihi and orange roughy. However, if occurrences were restricted to southern regions, areas of uncertainty were more prevalent (such as southern blue whiting), unless the background data incorporated climates at higher latitudes (such as hoki and ling).

A more restricted background method reduced the area in which the model was trained on and therefore increased the prevalence novel conditions in projected climates. The southern Blue Whiting predictions were an extreme example of this. Its occurrence records were only present in southern regions, and the base model had utilised a restricted background. Variables in the northern region were vastly different to the training data because the background points had only been taken from regions tightly encompassing and including the spread of its occurrence records. The result was that the northern half of the study region presented novel conditions not experienced during model training. The trevally base model also had a restricted background method applied. The result of this however was that there was more uncertainty in southern regions in its modern projection as the training data was all in northern regions. In contrast, ling and hoki MESS maps showed very little uncertainty in all projections, because the background sample used to train the base models encompassed the entire region.

Although selection of base models with restricted backgrounds created more uncertainty in some areas, this usually did not interfere with the model's ability to inform probability of presence. The uncertainty in northern regions for southern blue whiting and tarakihi did not necessarily undermine prediction interpretation for these species, both of which had high predicted probability of presence in southern regions, where there was relatively high certainty. Despite high uncertainty in northern regions, their absence there seemed likely based on the extent to which response curves described their thermal tolerance range (general pattern of decreasing at higher temps). 
Therefore, it is important to understand how the uncertainty estimates presented by the MESS maps influence projection interpretation in the context of the variable responses. For the species where their upper limits of their temperature tolerance range was captured by the model, their response to higher temperatures in future climates was more certain, such as tarakihi, trevally, ling and hoki where information on the upper bound of their temperature response was mostly if not completely available in the training data. Despite there being novel conditions in northern regions their response to higher temperatures is relatively obvious. These results suggest relatively high confidence in the projections of tarakihi, trevally, southern blue whiting, ling and hoki. But less so for the northern regions of snapper, john dory, orange roughy, arrow squid scampi and projections.

\section{3: Implications of Different Climate Scenarios}

For the fishery species included in the present study, the RCPS 2.6 usually resulted in the least change to their current distributions compared to other climate scenarios (trevally, tarakihi, arrow squid, orange roughy, john dory, and snapper). This is likely because the RCPS 2.6 is most similar to the modern environment compared to other scenarios. In this scenario sea temperatures increase around mid-century and subsequently decline slightly (Ministry for the Environment, 2018). For the RCPS 2.6 to occur, greenhouse gas emissions must begin to decline around 2020, and become negative by 2100. Many suggest that this is scenario is now unobtainable, and thus the RCPS 2.6 predictions in this study, are unlikely to occur.

RCPS 4.5 predictions often somewhat resembled the modern distributions, sometimes more so than in RCPS 2.6 (see hoki, ling, southern blue whiting, and scampi). This could be because the probability of presence of sub-antarctic species would increase more in response to the relatively small increase in surface temperatures in RCPS 4.5 than the slight decrease in RCPS 26. In RCPS 4.5, emissions begin to decline by 2040 but ocean warming continues, although at a lower rate than that of scenarios 6.0 and 8.5 (Ministry for the Environment, 2018). For most species the predictions in RCPS 4.5 differed more from modern distributions than the RCPS 2.6 predictions, (see orange roughy, trevally, tarakihi, arrow squid), often more so in 2100 (snapper, trevally, tarakihi, arrow squid). The RCPS 4.5 predictions in 2100 and 2050 were often indistinguishable or otherwise very similar (snapper, john dory, orange roughy, trevally, southern blue whiting, and hoki). This suggests stabilisation of distributions by 2050, which would reflect the stabilisation of greenhouse gas emissions required for this scenario to occur.

In RCPS 6.0 emissions peak in 2060 then begin to decline. Ocean temperatures are expected to continue to increase throughout the century (Ministry for the Environment, 2018). Although 2050 
predictions in RCPS 6.0 were often similar to those under RCPS 4.5, the 2100 predictions showed more substantial change from the modern projections. In 2100 predictions there was consistently more loss and contraction of suitable habitat than what was seen in either RCP 2.6 or 4.5 scenarios, (southern blue whiting, ling, hoki, arrow squid), more considerable range shifts (tarakihi, trevally), or more extreme expansions (john dory, snapper, orange roughy, scampi).

The 'business as usual scenario', RCPS 8.5 , in which emissions continue to rise indefinitely, always displayed the greatest changes observed for both 2050 and 2100 projections. Across almost all gases, RCPS 8.5 concentration levels are by far the highest throughout the century than any other climate scenario (Meinshausen et al., 2011). Furthermore, the 2100 predictions in this scenario were often vastly different from the 2050 ones, reflecting a lack of stabilisation in emission concentrations and an increasingly warming climate. Because this projected climate is very different to the modern climate, particularly in 2100, most uncertainty was consistently seen in RCP 8.52100 predictions.

The varied predicted responses of these species to climate changes were strongly influenced by the RCPS scenario. Which RCPS occurs is dependent on if and when greenhouse gas emissions decline (Van Vuuren et al., 2011). This implies that the availability of suitable habitat and future distributions for fishery species in New Zealand will be directly influenced by the response of humans to climate change. Although the RCPS 2.6 scenario may now be an unrealistic possibility, some still believe that RCP4.5 is possible, which is a stabilisation scenario that will result in less extreme changes, and therefore require less measurable response from fisheries management.

\section{5: Other information to inform models}

There are many factors not considered in the models built in this study that influence species distributions and how they respond to change. Low dispersal ability, reproductive rates, genetic diversity and/or overfishing, make populations more vulnerable to large climate shifts. Taking these factors into consideration may help to anticipate how species will respond and aid interpretation of predictions. Furthermore including some of these factors into the modelling process has been trailed with varied success as ability to do this is limited by available data, and complexity of the process (Miller \& Holloway, 2015; Sullivan et al., 2012; Zurell, 2017). Nonetheless, dispersal capability and biotic interaction information in particular could help to interpret predictions without directly informing the model building process, and thereby help to inform management decisions regarding the vulnerability of each species to changes in climate.

\section{Dispersal capability}


A species with high dispersal capability is more likely to be able to track suitable conditions as the climate changes. If it is unlikely that a species will be able to track suitable conditions, niche model projections may have over predicted range shifts in future climates. For example, snapper rarely exhibit long distance $(>100 \mathrm{~km})$ movement and dispersal is further limited by a short pelagic phase and high site fidelity (Bernal-Ramirez et al., 2003). Tarakihi however have been observed to move $300 \mathrm{~km}$ within a year, usually recaptured up to $200 \mathrm{~km}$ from the tagging site, and also have a relatively long pelagic larval stage (8-12 months) (Crossland 1976, Annala 1987, 1993; Smith 1989, Burridge \& Smolenski, 2003). Arrow squid also have high dispersal potential, with tagging experiments suggesting they travel on average about $1.1 \mathrm{~km}$ per day, and sometimes up to $5.6 \mathrm{~km}$ per day (Fisheries New Zealand, 2018). Therefore, shifts in tarakihi and arrow squid distributions are potentially likely to occur faster than snapper shifts.

Furthermore, inshore species are potentially more restricted in their dispersal capabilities than deep-water species due to human influence. Altered coastal landscapes, pollution and/or runoff from land, may limit a species ability to disperse or survive in certain regions. Further research to address how human alteration of the marine environment will affect species' ability to respond to climate change is pertinent.

\section{Biotic interactions}

Varied distribution shifts and rate of movement across species will likely result in changes in species interactions. Contraction of ranges to narrower areas will likely increase interspecific competition as well as intra-specific competition if multiple species contract to similar areas. Additionally, prey species may shift differently, or have different rates of response to their predators. For example, arrow squid is a food source for hoki, ling and many other species including marine mammals (Fisheries New Zealand, 2018). While hoki and ling were predicted to contract to the Chatham rise and the South Island's East Coast, arrow squid declined southward and thus has a different response to change its predators. These kinds of shifts could ultimately result in imbalances in the food chain. The predicted increase of scampi on the Chatham rise in future could be a positive input of prey for other species, such as ling (Fisheries New Zealand, 2018). Ultimately limited resources due to increased competition and/or differential shifts between prey and predator species could increase population vulnerabilities.

\section{6: Implications for Fisheries in New Zealand}

If the assumptions of this process are accepted and the predictions are relatively realistic, this study has important implications for fisheries management in New Zealand. Depending on the climate 
scenario that takes place, it is likely that fisheries in some areas will decline, while in fisheries new areas may emerge. Harvesting, particularly overfishing of species that are already predicted to decline, would make them more vulnerable to the stress of degraded environmental conditions from climate change effects. For example, tarakihi and trevally are predicted to decline from waters around the North Island. While the longevity of the trevally fishery could be compromised, new fisheries may emerge further southward for tarakihi. New fisheries around the South Island may also emerge for snapper and john dory, with possible simultaneous increase in productivity in northern regions. Projections about suitable conditions for fishery species could therefore inform management and help determine the adjustments that would be required for catch quotas in different regions.

Although, these are predictions for what distributions may be like in $50-100$ years, it is important to start considering the implications of climate change as soon as possible. This would best be served by management that anticipates what change is likely and proactively responds to it. In the long term, a proactive response to changes would provide benefits for the fishery industry and help secure the sustainable harvesting of New Zealand fisheries. However, in industry and political management response is often constrained to short term goals and foresights, as well as general scepticism of future modelling strategies.

It is important to remember that these models are not about describing everything. There are complex interactions that cannot be modelled, but the aim is to get a better idea of the future by drawing from simple but reliable modelled correlations. Forward modelling can narrow the range of expectations for what change might look like. If government and society is unresponsive about 2100 , we may risk over-harvesting populations, not adjusting appropriately to utilising shifting resources, and creating conflict over management boundaries as catch rates and productive areas change. Long term thinking about these changes would be beneficial, particularly as model reliability improves and as the future direction of climate change becomes more certain. Ultimately, more information can lead to better decisions about the future, and not including predictive modelling would be an oversight.

The risks of not responding are particularly high if emissions are not reduced and the RCPS 8.5 becomes reality. The consequence of this will be especially high for species that experience degraded area of suitable conditions and declining probability of presence, such as trevally, southern blue whiting, hoki, and ling. The continued harvest of these species in a similar way as what is currently done would likely be unsustainable and lead to population depletions. Conversely, responding well could include increasing catch quotas in some regions for species that increase such 
as snapper. Inaction could thus be a waste of a new valuable economic resource. Stronger harvest of sub-tropical or temperature species that are predicted to increase could also mitigate some competition from these species for those that decline, and their encroachment on more southern species habitats.

In order to avoid large changes to management of fishery species in New Zealand, or unsustainable harvest of species in decline, a less extreme climate scenario would be preferable such as RCPS 2.6 or 4.5. Without global efforts to reduce greenhouse gas emissions, there are deep concerns that the human race will not respond in time, and these scenarios will be bypassed for more extreme ones where ecosystems are majorly affected (Schellnhuber et al. 2016; Steffen et al., 2018).

\section{7: Future Directions}

\section{Model improvement}

Careful covariate selection is required but difficult because relevance of each varies with species. In this work, the same variables were used for all species for a parsimonious approach. Although there are many other climate variables not used in this study that are associated with marine species distributions, many of these are correlated with temperature (Cheung et al., 2016). Warming is likely the best indication of shifts (Cheung et al., 2009; Robinson et al., 2015), but other factors such as depth (Rutterford et al., 2015) or salinity (Weinert et al., 2016) or Net Primary Productivity (NPP) (Hazen et al., 2013), may constrain these shifts (Morley et al., 2018a). NPP was not included in this study but NPP influences many processes and interactions in the marine environment. NPP changes are predicted to be small but positive in sub-antarctic waters and larger but negative in subtropical waters (Pinkerton et al., 2016). NPP in all regions around New Zealand is predicted to decrease in the future as a result of climate change, with the exception of the Chatham Rise and sub-antarctic areas (Pinkerton et al., 2016). Despite the potential usefulness of this information, the more variables that are included, the more complex the models are, and the more uncertain and over parametrized they become. This is a common and largely unavoidable issue for most methods that attempt to model the natural world. Therefore, it would be ideal to create species-specific variable sets as was done by Zeng et al. (2016). This would allow the addition of more variables without making models too complex (Zeng et al., 2016).

The climate data used in this study were 5-arc min and were subset from worldwide climate datasets (Assis et al., 2018). Coverage of inshore areas is likely poorer than other areas as there are usually details missing that heavily influence these environments. Higher quality inshore data could therefore improve projections, particularly for coastal species (Freer et al., 2018). The addition of 
data records from other regions could also be useful in helping to predict the species distributions where full response to some variables was not captured by the models (discussed in section 4.2). For now, the variables and records used in this study provide a 'best guess' of how future climate change might affect these fishery species.

\section{Data to corroborate research results}

Future forecasts are difficult to substantiate. Combining and/or comparing several modelling approaches is a good way to asses model credibility (Elith et al., 2010; Jones \& Cheung (2014); Thuiller et al., 2005). Using different ENM algorithms to model the response to future climates of the species modelled here would help to verify or refute the predictions made. Different algorithms that produce similar results would increase prediction credibility.

Future monitoring of species distribution changes would also provide information on how accurate these predictions are. Currently no systems are in place in New Zealand to document catch findings in a collaborative way, and large-scale surveys are heavily relied upon to monitor movements. Anecdotal evidence of these species may help to corroborate or dispute findings, particularly for popular recreational fishery species. Anecdotal evidence is a valuable tool in the absence of frequent monitoring of populations and their movements. Australia has an example of site where the public can document catches and unusual findings (http://www.redmap.org.au/). A similar system in New Zealand has not yet been as widely popularised (https://www.fish4all.co.nz/about-us). This sort of documentation if implemented in future could help to corroborate or contradict climate change response predictions. Furthermore, there is value in considering how multiple species are responding rather than just a few. If some species shift, it is likely others will too. There is anecdotal evidence of other fish species moving southward in New Zealand recently such as Kahwai and Kingfish (Lewis, 2018). Future modelling and monitoring of multiple species will contribute information to the overall way in which biodiversity will change around New Zealand. 


\section{Chapter 5: Conclusions}

This work has highlighted the dynamic and variable responses of species' distributions responding to changing climates. There was considerable variation between species in distance moved over time and the associated change in area of highly suitable conditions. Expansion or shifts typically followed a southward trend but the way that the suitable area changed varied, even between species that followed poleward trends. Obvious effects of extremely different climates in New Zealand tended to be one of three things; the range contracted (southern blue whiting, ling and hoki), the range expanded (snapper, john dory, orange roughy), or the range shifted (tarakihi, arrow squid, scampi). For trevally this effect was both a shift and contraction of their range.

This work has achieved four key outcomes:

- Provided a possible glimpse into the future distributions of species for four different future climate scenarios to suggest those in management consider these possible shifts and improve or implement long term management strategies. Additionally, it has shown that different species respond differently to change and to different extents.

- Provided support for published studies, which have already shown poleward shifts for marine species in temperate and subtropical waters and higher species turnover near the poles. Many species in New Zealand will likely reflect this global pattern.

- Emphasised the importance of and provided an incentive for collaborative scientific effort for better collection and documentation of occurrence records from anecdotal catches and large-scale survey efforts over multiple countries. Anecdotal evidence is valuable for providing information that could inform and/or corroborate ENM studies, and formalising or collecting anecdotal evidence in a cohesive way would be useful.

- Provided incentive to keep mean global temperatures down and reduce greenhouse gas emissions as this will result in less species turnover, smaller distribution shifts, and less biodiversity loss (Cheung et al., 2016). This would require less large-scale management changes. Demonstrating the benefits of reducing greenhouse gas emissions may encourage commitment to more ambitious plans to seriously address climate change and promote faster action. 


\section{References}

Aiello-Lammens, M. E., Hanson, J. O., Boria, R. A., Radosavljevic, A., Vilela, B., \& Anderson, R. P. (2015(a)). spThin (version 1.0.0): Functions to spatially rarefy and thin species occurrence data.

Aiello-Lammens, M. E., Boria, R. A., Radosavljevic, A., Vilela, B., \& Anderson, R. P. (2015(b)). spThin: an $\mathrm{R}$ package for spatial thinning of species occurrence records for use in ecological niche models. Ecography, 38(5), 541-545.

Alabia, I. D., Saitoh, S.-I., Igarashi, H., Ishikawa, Y., Usui, N., Kamachi, M., ... Seito, M. (2015). Future projected impacts of ocean warming to potential squid habitat in western and central North Pacific. Ices Journal of Marine Science, 73(5), 1343-1356.

Allen, G. R., \& Heemstra, P. C. (1976). Cheilodactylus rubrolabiatus, a new species of morwong (Pisces: Cheilodactylidae) from Western Austrailia, with a new key to the Cheilodactylid fishes of Austrailia. (Vol. 4).

Alyling, T., \& Cox, G. J. (1982). Collins Guide to the Deep Sea Fishes of New Zealnad Auckland: Collins.

Anderson, R. P. (2003). Real vs. artefactual absences in species distributions: tests for Oryzomys albigularis (Rodentia: Muridae) in Venezuela. Journal of Biogeography, 30(4), 591-605.

Anderson, R. P. (2015). Modeling niches and distributions: it's not just "click, click, click". BIOGEOGRAFÍA 8, 11.

Anderson, R. P., \& Gonzalez, I., Jr. (2011). Species-specific tuning increases robustness to sampling bias in models of species distributions: An implementation with Maxent. Ecological Modelling, 222(15), 2796-2811. doi:10.1016/j.ecolmodel.2011.04.011

Anderson, R. P., Lew, D., \& Peterson, A. T. (2003). Evaluating predictive models of species' distributions: criteria for selecting optimal models. Ecological Modelling, 162(3), 211-232.

Anderson, R. P., \& Raza, A. (2010). The effect of the extent of the study region on GIS models of species geographic distributions and estimates of niche evolution: preliminary tests with montane rodents (genus Nephelomys) in Venezuela. Journal of Biogeography, 37(7), 13781393.

Andrews, A., \& Tracey, D. (2003). Age validation of deepwater fish species, with particular reference to New Zealand orange roughy, black oreo, smooth oreo, and black cardinalfish. Retrieved from

Andrews, A. H., Tracey, D. M., \& Dunn, M. R. (2009). Lead-radium dating of orange roughy (Hoplostethus atlanticus): validation of a centenarian life span. Canadian Journal of Fisheries and Aquatic Sciences, 66(7), 1130-1140.

Ashton, D. T., Hilario, E., Jaksons, P., Ritchie, P. A., \& Wellenreuther, M. (2019). Genetic diversity and heritability of economically important traits in captive Australasian snapper (Chrysophrys auratus). Aquaculture, 505, 190-198. doi:10.1016/j.aquaculture.2019.02.034

Assis, J., Tyberghein, L., Bosch, S., Verbruggen, H., Serrao, E. A., \& De Clerck, O. (2018). Bio-ORACLE v2.0: Extending marine data layers for bioclimatic modelling. Global Ecology and Biogeography, 27(3), 277-284. doi:10.1111/geb.12693

Assis, J., Tyberghein, L., Bosch, S., Verbruggen, H., Serrão, E. A., \& De Clerck, O. (2018). Bio-ORACLE v2. 0: Extending marine data layers for bioclimatic modelling. Global Ecology and Biogeography, 27(3), 277-284.

Baasch, D. M., Tyre, A. J., Millspaugh, J. J., Hygnstrom, S. E., \& Vercauteren, K. C. (2010). An evaluation of three statistical methods used to model resource selection. Ecological Modelling, 221(4), 565-574.

Balzano, S., Sarno, D., \& Kooistra, W. H. C. F. (2010). Effects of salinity on the growth rate and morphology of ten Skeletonema strains. Journal of Plankton Research, 33(6), 937-945. doi:10.1093/plankt/fbq150 
Barange, M., King, J., Valdes, L., \& Turra, A. (2016). The evolving and increasing need for climate change research on the oceans. Ices Journal of Marine Science, 73(5), 1267-1271. doi:10.1093/icesjms/fsw052

Barros, V., Field, C., Dokke, D., Mastrandrea, M., Mach, K., Bilir, T., . . Genova, R. (2014). Climate change 2014: impacts, adaptation, and vulnerability-Part B: regional aspects-Contribution of Working Group II to the Fifth Assessment Report of the Intergovernmental Panel on Climate Change.

Barve, N., Barve, V., Jimenez-Valverde, A., Lira-Noriega, A., Maher, S. P., Peterson, A. T., . . . Villalobos, F. (2011). The crucial role of the accessible area in ecological niche modeling and species distribution modeling. Ecological Modelling, 222(11), 1810-1819. doi:10.1016/j.ecolmodel.2011.02.011

Barve, N., Barve, V., Jiménez-Valverde, A., Lira-Noriega, A., Maher, S. P., Peterson, A. T., . . Villalobos, F. (2011). The crucial role of the accessible area in ecological niche modeling and species distribution modeling. Ecological Modelling, 222(11), 1810-1819.

Beck, L. (2018). The Baltic Sea offers a worrying preview of what's to come with global warming.

Beentjes, M. (2011). TAR 3 catch sampling in 2009-10 and fishery characterisation (1989-90 to 2009-10). New Zealand Fisheries Assessment Report, 52.

Beentjes, M. P., \& Stevenson, M. L. (2008). Inshore trawl survey of Canterbury Bight and Pegasus Bay, May-June 2007 (KAH0705). New Zealand Fisheries Assessment Report, 38, 95.

Bell, M., Tuck, I., \& Dobby, H. (2013). Nephrops species. Lobsters: biology, management, aquaculture and fisheries, 357-413.

Bell, R. J., Richardson, D. E., Hare, J. A., Lynch, P. D., \& Fratantoni, P. S. (2014). Disentangling the effects of climate, abundance, and size on the distribution of marine fish: an example based on four stocks from the Northeast US shelf. Ices Journal of Marine Science, 72(5), 1311-1322.

Bernal-Ramirez, J. H., Adcock, G. J., Hauser, L., Carvalho, G. R., \& Smith, P. J. (2003). Temporal stability of genetic population structure in the New Zealand snapper, Pagrus auratus, and relationship to coastal currents. Marine Biology, 142(3), 567-574. doi:10.1007/s00227-0020972-9

Blanchard, J. L., Jennings, S., Holmes, R., Harle, J., Merino, G., Allen, J. I., . . Barange, M. (2012). Potential consequences of climate change for primary production and fish production in large marine ecosystems. Philosophical Transactions of the Royal Society B: Biological Sciences, 367(1605), 2979-2989.

Booth, T. (1985). A new method for assisting species selection. The Commonwealth Forestry Review, 241-250.

Booth, T. H., Nix, H. A., Busby, J. R., \& Hutchinson, M. F. (2014). BIOCLIM: the first species distribution modelling package, its early applications and relevance to most current MAXENT studies. Diversity and Distributions, 20(1), 1-9.

Boria, R. A., Olson, L. E., Goodman, S. M., \& Anderson, R. P. (2014). Spatial filtering to reduce sampling bias can improve the performance of ecological niche models. Ecological Modelling, 275, 73-77.

Branch, T. A. (2001). A review of orange roughy Hoplostethus atlanticus fisheries, estimation methods, biology and stock structure. South African Journal of Marine Science-SuidAfrikaanse Tydskrif Vir Seewetenskap, 23, 181-203.

Breece, M. W., Fox, D. A., Dunton, K. J., Frisk, M. G., Jordaan, A., \& Oliver, M. J. (2016). Dynamic seascapes predict the marine occurrence of an endangered species: Atlantic Sturgeon Acipenser oxyrinchus oxyrinchus. Methods in Ecology and Evolution, 7(6), 725-733.

Brewer, M. J., Butler, A., \& Cooksley, S. L. (2016). The relative performance of AIC, AICC and BIC in the presence of unobserved heterogeneity. Methods in Ecology and Evolution, 7(6), 679-692. doi:10.1111/2041-210x.12541 
Bruge, A., Alvarez, P., Fontán, A., Cotano, U., \& Chust, G. (2016). Thermal niche tracking and future distribution of Atlantic mackerel spawning in response to ocean warming. Frontiers in Marine Science, 3, 86.

Burnham, K. P., \& Anderson, D. R. (2004). Multimodel inference: understanding AIC and BIC in model selection. Sociological methods \& research, 33(2), 261-304.

Burridge, C. P., \& Smolenski, A. J. (2003). Lack of genetic divergence found with microsatellite DNA markers in the tarakihi Nemadactylus macropterus. New Zealand Journal of Marine and Freshwater Research, 37(2), 223-230. doi:10.1080/00288330.2003.9517160

Carneiro, L. R. d. A., Lima, A. P., Machado, R. B., \& Magnusson, W. E. (2016). Limitations to the use of species-distribution models for environmental-impact assessments in the Amazon. Plos One, 11(1), e0146543.

Carpenter, G., Gillison, A., \& Winter, J. (1993). DOMAIN: a flexible modelling procedure for mapping potential distributions of plants and animals. Biodiversity \& Conservation, 2(6), 667-680.

Carr, M. H., Neigel, J. E., Estes, J. A., Andelman, S., Warner, R. R., \& Largier, J. L. (2003). COMPARING MARINE AND TERRESTRIAL ECOSYSTEMS: IMPLICATIONS FOR THE DESIGN OF COASTAL MARINE RESERVES. Ecological Applications, 13(sp1), 90-107. doi:10.1890/10510761(2003)013[0090:Cmatei]2.0.Co;2

Carsten, F. D., Jana, M. M., Miguel, B. A., Roger, B., Janine, B., Gudrun, C., . . Robert, W. (2007). Methods to account for spatial autocorrelation in the analysis of species distributional data: a review. Ecography, 30(5), 609-628. doi:doi:10.1111/j.2007.0906-7590.05171.x

Caton, A., \& McLoughlin, K. (2000). Fishery Status Reports 1999: Resource Assessments of Australian Commonwealth Fisheries: Bureau of Rural Sciences.

Cheng, L., Abraham, J., Hausfather, Z., \& Trenberth, K. E. (2019). How fast are the oceans warming? Science, 363(6423), 128-129.

Cheung, W. W., Meeuwig, J. J., Feng, M., Harvey, E., Lam, V. W., Langlois, T., . . Pauly, D. (2012). Climate-change induced tropicalisation of marine communities in Western Australia. Marine and Freshwater Research, 63(5), 415-427.

Cheung, W. W. L., Frolicher, T. L., Asch, R. G., Jones, M. C., Pinsky, M. L., Reygondeau, G., . . Watson, J. R. (2016). Building confidence in projections of the responses of living marine resources to climate change. Ices Journal of Marine Science, 73(5), 1283-1296. doi:10.1093/icesjms/fsv250

Cheung, W. W. L., Lam, V. W. Y., Sarmiento, J. L., Kearney, K., Watson, R., \& Pauly, D. (2009). Projecting global marine biodiversity impacts under climate change scenarios. Fish and Fisheries, 10(3), 235-251. doi:10.1111/j.1467-2979.2008.00315.x

Cheung, W. W. L., Reygondeau, G., \& Frölicher, T. L. (2016). Large benefits to marine fisheries of meeting the $1.5^{\circ} \mathrm{C}$ global warming target. Science, 354(6319), 1591-1594. doi:10.1126/science.aag2331

Cheung, W. W. L., Watson, R., \& Pauly, D. (2013). Signature of ocean warming in global fisheries catch. Nature, 497, 365. doi:10.1038/nature12156

Chikerema, S. M., Gwitira, I., Murwira, A., Pfukenyi, D. M., \& Matope, G. (2017). Comparison of GARP and Maxent in modelling the geographic distribution of Bacillus anthracis in Zimbabwe. Zimbabwe Veterinary Journal, 35(1), 1-6.

Christian, J. R., \& Holmes, J. (2016). Changes in albacore tuna habitat in the northeast Pacific Ocean under anthropogenic warming. Fisheries Oceanography, 25(5), 544-554. doi:10.1111/fog.12171

Cohen, D. M., \& Nielsen, J. G. (1978). Guide to the identification of genera of fish order Ophidiiformes with tentative classification of the order. Retrieved from

Colautti, R. I., \& Barrett, S. C. H. (2013). Rapid Adaptation to Climate Facilitates Range Expansion of an Invasive Plant. Science, 342(6156), 364-366. doi:10.1126/science.1242121 
Connor, T., Hull, V., Viña, A., Shortridge, A., Tang, Y., Zhang, J., . . Liu, J. (2018). Effects of grain size and niche breadth on species distribution modeling. Ecography, 41(8), 1270-1282. doi:doi:10.1111/ecog.03416

Corkeron, P. J., Minton, G., Collins, T., Findlay, K., Willson, A., \& Baldwin, R. (2011). Spatial models of sparse data to inform cetacean conservation planning: an example from Oman. Endangered Species Research, 15(1), 39-52.

Costello, M. J., Coll, M., Danovaro, R., Halpin, P., Ojaveer, H., \& Miloslavich, P. (2010). A census of marine biodiversity knowledge, resources, and future challenges. Plos One, 5(8), e12110.

Crozier, L. G., \& Hutchings, J. A. (2014). Plastic and evolutionary responses to climate change in fish. Evolutionary Applications, 7(1), 68-87.

Cryer, M., \& Oliver, M. (2001). Estimating age and growth in New Zealand scampi, Metanephrops challengeri. Retrieved from

D'heygere, T., Goethals, P. L., \& De Pauw, N. (2006). Genetic algorithms for optimisation of predictive ecosystems models based on decision trees and neural networks. Ecological Modelling, 195(1-2), 20-29.

Dambach, J., \& Rödder, D. (2011). Applications and future challenges in marine species distribution modeling. Aquatic Conservation: Marine and Freshwater Ecosystems, 21(1), 92-100. doi:10.1002/aqc.1160

De Marco Junior, P., \& Nobrega, C. C. (2018). Evaluating collinearity effects on species distribution models: An approach based on virtual species simulation. Plos One, 13(9). doi:10.1371/journal.pone.0202403

Derville, S., Torres, L. G., lovan, C., \& Garrigue, C. (2018). Finding the right fit: Comparative cetacean distribution models using multiple data sources and statistical approaches. Diversity and Distributions, 24(11), 1657-1673.

Donelson, J. M., Sunday, J. M., Figueira, W. F., Gaitán-Espitia, J. D., Hobday, A. J., Johnson, C. R., . . Pandolfi, J. M. (2019). Understanding interactions between plasticity, adaptation and range shifts in response to marine environmental change. Philosophical Transactions of the Royal Society B, 374(1768), 20180186.

Dudik, M., Phillips, S. J., \& Schapire, R. E. (2007). Maximum entropy density estimation with generalized regularization and an application to species distribution modeling. Journal of Machine Learning Research, 8, 1217-1260.

Dudík, M., Phillips, S. J., \& Schapire, R. E. (2006). Correcting sample selection bias in maximum entropy density estimation. Paper presented at the Advances in neural information processing systems.

Dunford, A. J., O'Driscoll, R. L., \& Oeffner, J. (2015). Improvements in estimating an acoustic target strength-length relationship for hoki (Macruronus novaezeladiae). Fisheries Research, 162, 12-19. doi:10.1016/j.fishres.2014.09.008

Dunn, M., Rickard, G., Sutton, P., \& Doonan, I. (2009). Nursery grounds of the orange roughy around New Zealand. Ices Journal of Marine Science, 66(5), 871-885.

Dunn, M. R. (2009). Feeding habits of the ommastrephid squid Nototodarus sloanii on the Chatham Rise, New Zealand. New Zealand Journal of Marine and Freshwater Research, 43(5), 11031113. doi:10.1080/00288330.2009.9626533

Dunn, M. R., Connell, A. M., Forman, J., Stevens, D. W., \& Horn, P. L. (2010). Diet of two large sympatric teleosts, the ling (Genypterus blacodes) and hake (Merluccius australis). Plos One, 5(10), e13647.

Dunn, M. R., \& Jones, E. G. (2013). Stock structure and fishery characterisation for New Zealand John dory: Ministry for Primary Industries.

Elith, J., \& Graham, C. H. (2009). Do they? How do they? WHY do they differ? On finding reasons for differing performances of species distribution models. Ecography, 32(1), 66-77. 
Elith, J., Graham, C. H., Anderson, R. P., Dudík, M., Ferrier, S., Guisan, A., . . Lehmann, A. (2006). Novel methods improve prediction of species' distributions from occurrence data. Ecography, 129-151.

Elith, J., Kearney, M., \& Phillips, S. (2010). The art of modelling range-shifting species. Methods in Ecology and Evolution, 1(4), 330-342.

Elith, J., \& Leathwick, J. (2007). Predicting species distributions from museum and herbarium records using multiresponse models fitted with multivariate adaptive regression splines. Diversity and Distributions, 13(3), 265-275.

Elith, J., Phillips, S. J., Hastie, T., Dudík, M., Chee, Y. E., \& Yates, C. J. (2011). A statistical explanation of MaxEnt for ecologists. Diversity and Distributions, 17(1), 43-57.

Fielder, D. S., Bardsley, W. J., Allan, G. L., \& Pankhurst, P. M. (2005). The effects of salinity and temperature on growth and survival of Australian snapper, Pagrus auratus larvae. Aquaculture, 250(1-2), 201-214.

Fisheries New Zealand (2018). Fisheries Assessment Plenary, May 2018: stock assessments and stock status. Compiled by the Fisheries Science and Information Group, Fisheries New Zealand, Wellington, New Zealand.

Fithian, W., Elith, J., Hastie, T., \& Keith, D. A. (2015). Bias correction in species distribution models: pooling survey and collection data for multiple species. Methods in Ecology and Evolution, 6(4), 424-438.

Fitzpatrick, M. C., Gotelli, N. J., \& Ellison, A. M. (2013). MaxEnt versus MaxLike: empirical comparisons with ant species distributions. Ecosphere, 4(5), 1-15.

Fourcade, Y., Engler, J. O., Rödder, D., \& Secondi, J. (2014). Mapping species distributions with MAXENT using a geographically biased sample of presence data: a performance assessment of methods for correcting sampling bias. PLOS ONE, 9(5), e97122.

Francis, M. P. (1993). Does water temperature determine year class strength in New Zealand snapper (Pagrus auratus, Sparidae)? Fisheries Oceanography, 2(2), 65-72.

Free, C. M., Thorson, J. T., Pinsky, M. L., Oken, K. L., Wiedenmann, J., \& Jensen, O. P. (2019). Impacts of historical warming on marine fisheries production. Science, 363(6430), 979-983. doi:10.1126/science.aau1758

Freer, J. J., Partridge, J. C., Tarling, G. A., Collins, M. A., \& Genner, M. J. (2018). Predicting ecological responses in a changing ocean: the effects of future climate uncertainty. Marine Biology, 165(1), 7-7. doi:10.1007/s00227-017-3239-1

Frost, M., Baxter, J., Buckley, P., Dye, S., \& Stoker, B. (2017). Reporting marine climate change impacts: Lessons from the science-policy interface. Environmental Science \& Policy, 78, 114120. doi:10.1016/j.envsci.2017.10.003

Fukuda, S., De Baets, B., Waegeman, W., Verwaeren, J., \& Mouton, A. M. (2013). Habitat prediction and knowledge extraction for spawning European grayling (Thymallus thymallus L.) using a broad range of species distribution models. Environmental modelling \& software, 47, 1-6.

Gavin, D. G., Fitzpatrick, M. C., Gugger, P. F., Heath, K. D., Rodríguez-Sánchez, F., Dobrowski, S. Z., . . Bartlein, P. J. (2014). Climate refugia: joint inference from fossil records, species distribution models and phylogeography. New Phytologist, 204(1), 37-54.

Giovanelli, J. G., de Siqueira, M. F., Haddad, C. F., \& Alexandrino, J. (2010). Modeling a spatially restricted distribution in the Neotropics: How the size of calibration area affects the performance of five presence-only methods. Ecological Modelling, 221(2), 215-224.

Gobeyn, S., Mouton, A. M., Cord, A. F., Kaim, A., Volk, M., \& Goethals, P. L. (2019). Evolutionary algorithms for species distribution modelling: A review in the context of machine learning. Ecological Modelling, 392, 179-195.

Golikov, A., Sabirov, R., Lubin, P., \& Jørgensen, L. (2013). Changes in distribution and range structure of Arctic cephalopods due to climatic changes of the last decades. Biodiversity, 14(1), 28-35. 
Graham, C. H., Moritz, C., \& Williams, S. E. (2006). Habitat history improves prediction of biodiversity in rainforest fauna. Proceedings of the National Academy of Sciences, 103(3), 632-636.

Graham, C. H., Ron, S. R., Santos, J. C., Schneider, C. J., \& Moritz, C. (2004). Integrating phylogenetics and environmental niche models to explore speciation mechanisms in dendrobatid frogs. Evolution, 58(8), 1781-1793. doi:10.1554/03-274

Grieve, B. D., Curchitser, E. N., \& Rykaczewski, R. R. (2016). Range expansion of the invasive lionfish in the Northwest Atlantic with climate change. Marine Ecology Progress Series, 546, 225237.

Hamer, P. A., Kemp, J., Robertson, S., \& Hindell, J. S. (2012). Multiple otolith techniques aid stock discrimination of a broadly distributed deepwater fishery species, blue grenadier, Macruronus novaezelandiae. Fisheries Research, 113(1), 21-34. doi:10.1016/j.fishres.2011.08.016

Hanchet, S. (1999). Stock structure of southern blue whiting (Micromesistius australis) in New Zealand waters. New Zealand Journal of Marine and Freshwater Research, 33(4), 599-609.

Hare, J. A., Alexander, M. A., Fogarty, M. J., Williams, E. H., \& Scott, J. D. (2010). Forecasting the dynamics of a coastal fishery species using a coupled climate-population model. Ecological Applications, 20(2), 452-464.

Hare, J. A., Wuenschel, M. J., \& Kimball, M. E. (2012). Projecting range limits with coupled thermal tolerance-climate change models: an example based on gray snapper (Lutjanus griseus) along the US east coast. Plos One, 7(12), e52294.

Hazen, E. L., Jorgensen, S., Rykaczewski, R. R., Bograd, S. J., Foley, D. G., Jonsen, I. D., . . Crowder, L. B. (2013). Predicted habitat shifts of Pacific top predators in a changing climate. Nature Climate Change, 3(3), 234.

Heemstra, P. C. (1980). A revision of the zeid fishes (Zeiformes: Zeidae) of South Africa (Vol. 41).

Hiddink, J., \& Ter Hofstede, R. (2008). Climate induced increases in species richness of marine fishes. Global Change Biology, 14(3), 453-460.

Hijmans, R. J., \& Elith, J. (2013). Species distribution modeling with R. R package version 0.8-11.

Hijmans, R. J., Garrett, K. A., Huamán, Z., Zhang, D. P., Schreuder, M., \& Bonierbale, M. (2000). Assessing the Geographic Representativeness of Genebank Collections: the Case of Bolivian Wild Potatoes. Conservation Biology, 14(6), 1755-1765. doi:doi:10.1111/j.15231739.2000.98543.x

Hijmans, R. J., Phillips, S., Leathwick, J. and Elith, J. (2011). Package 'dismo'. Available online at: http://cran.r-project.org/web/packages/dismo/index.html.

Hoffmann, A. A., \& Sgro, C. M. (2011). Climate change and evolutionary adaptation. Nature, 470(7335), 479.

Hofmann, M., \& Schellnhuber, H.-J. (2009). Oceanic acidification affects marine carbon pump and triggers extended marine oxygen holes. Proceedings of the National Academy of Sciences, 106(9), 3017-3022. doi:10.1073/pnas.0813384106

Horn, P. (2005). A review of the stock structure of ling (GenHterus blacodes) in New Zealand waters. New Zealand Fisheries Assessment Report, 59.

Hurst, R., Bagley, N., Chatterton, T., Hanchet, S., Schofield, K., \& Vignaux, M. (1992). Standardisation of hoki/middle depth time series trawl surveys. MAF Fisheries Greta Point Internal Report No. 194. 89 p. Unpublished report held in NIWA library, Wellington.

Ismen, A., Arslan, M., Yigin, C., \& Bozbay, N. (2013). Age, growth, reproduction and feeding of J ohn $D$ ory, $Z$ eus faber ( $P$ isces: $Z$ eidae), in the $S$ aros $B$ ay ( $N$ orth $A$ egean $S$ ea). Journal of Applied Ichthyology, 29(1), 125-131.

Jereb, P., \& Roper, C. F. (2005). Cephalopods of the World: Myopsid and Oegopsid squids (Vol. 2): Fao. 
Jones, Miranda C. \& Cheung, William W. L. (2014). Multi-model ensemble projections of climate change effects on global marine biodiversity. ICES Journal of Marine Science, 72(3), 741-752.

Jung, S., Pang, I. C., Lee, J. H., Choi, I., \& Cha, H. K. (2014). Latitudinal shifts in the distribution of exploited fishes in Korean waters during the last 30 years: a consequence of climate change. Reviews in Fish Biology and Fisheries, 24(2), 443-462. doi:10.1007/s11160-013-9310-1

Kadmon, R., Farber, O., \& Danin, A. (2004). Effect of roadside bias on the accuracy of predictive maps produced by bioclimatic models. Ecological Applications, 14(2), 401-413.

Kaschner, K., Watson, R., Trites, A., \& Pauly, D. (2006). Mapping world-wide distributions of marine mammal species using a relative environmental suitability (RES) model. Marine Ecology Progress Series, 316, 285-310.

Khan, A. H., Chmura, G. L., \& Levac, E. (2013). Future sea surface temperatures in Large Marine Ecosystems of the Northwest Atlantic. Ices Journal of Marine Science, 70(5), 915-921. doi:10.1093/icesjms/fst002

Kimmerer, W. (2002). Effects of freshwater flow on abundance of estuarine organisms: physical effects or trophic linkages? Marine Ecology Progress Series, 243, 39-55.

Kulka, D., Themelis, D., \& Halliday, R. (2003). Orange roughy (Hoplostethus atlanticus Collett 1889) in the Northwest Atlantic. Journal of Northwest Atlantic Fishery Science, 31, 47.

Kültz, D. (2015). Physiological mechanisms used by fish to cope with salinity stress. Journal of Experimental Biology, 218(12), 1907-1914.

Laptikhovsky, V. (2008). The first record of orange roughy (Hoplostethus atlanticus) in the southwest Atlantic. Marine Biodiversity Records, 1.

Law, C. S., Bell, J. J., Bostock, H. C., Cornwall, C. E., Cummings, V. J., Currie, K., Davy, S. K., Gammon, M., Hepburn, C. D., Hurd, C. L., Lamare, M., Mikaloff-Fletcher, S. E., Nelson, W. A., Parsons, D. M., Ragg, N. L. C., Sewell, M. A., Smith, A. M., Tracey, D. M. (2017). Ocean acidification in New Zealand waters: trends and impacts. New Zealand Journal of Marine and Freshwater Research, 1-41.

Lehtonen, T. K., Wong, B. B. M., \& Kvarnemo, C. (2016). Effects of salinity on nest-building behaviour in a marine fish. BMC ecology, 16, 7-7. doi:10.1186/s12898-016-0067-y

Lewis, J. (2018). Explosion in fish numbers as sea warms. Retrieved from https://www.nzherald.co.nz/nz/news/article.cfm?c id=1\&objectid=11979914

Lindsay, R. E., Constantine, R., Robbins, J., Mattila, D. K., Tagarino, A., \& Dennis, T. E. (2016). Characterising essential breeding habitat for whales informs the development of large-scale Marine Protected Areas in the South Pacific. Marine Ecology Progress Series, 548, 263-275.

Lobo, J. M., Jiménez-Valverde, A., \& Real, R. (2008). AUC: a misleading measure of the performance of predictive distribution models. Global Ecology and Biogeography, 17(2), 145-151.

Mackay, K. A. (2011). Database documentation: trawl. NIWA Fisheries Data Management Database Documentation Series

Major, R. N., \& Jeffs, A. G. (2018). Laboratory comparison of potential natural baits for potting New Zealand scampi, Metanephrops challengeri. Bulletin of Marine Science, 94(3), 635-655.

Mannocci, L., Roberts, J. J., Halpin, P. N., Authier, M., Boisseau, O., Bradai, M. N., . . Vella, J. (2018). Assessing cetacean surveys throughout the Mediterranean Sea: a gap analysis in environmental space. Scientific Reports, 8. doi:10.1038/s41598-018-19842-9

Manzoor, S. A., Griffiths, G., \& Lukac, M. (2018). Species distribution model transferability and model grain size - finer may not always be better. Scientific Reports, 8(1), 7168. doi:10.1038/s41598-018-25437-1

Maravelias, C., Tsitsika, E., \& Papaconstantinou, C. (2007). Seasonal dynamics, environmental preferences and habitat selection of john dory (Zeus faber). Estuarine, Coastal and Shelf Science, 72(4), 703-710. 
Martinez-Meyer, E., Townsend Peterson, A., \& Hargrove, W. W. (2004). Ecological niches as stable distributional constraints on mammal species, with implications for Pleistocene extinctions and climate change projections for biodiversity. Global Ecology and Biogeography, 13(4), 305-314. doi:10.1111/j.1466-822X.2004.00107.x

Mateo, R. G., Croat, T. B., Felicísimo, Á. M., \& Muñoz, J. (2010). Profile or group discriminative techniques? Generating reliable species distribution models using pseudo-absences and target-group absences from natural history collections. Diversity and Distributions, 16(1), 8494.

May, G. (2019). Marine heatwave: Hawke's Bay's scorching seas, and the potential impacts.

McKenzie, A. (2017). Assessment of hoki (Macruronus novaezelandiae) in 2016. Ministry for Primary Industries.

McKenzie, J. R., Beentjes, M. P., Parker, S. J., Parsons, D. M., Armiger, H., Wilson, O., . . Walsh, C. (2017). Fishery Characterisation and Age Composition of tarakihi in TAR 1, 2 and 3 for 2013/14 and 2014/15: Ministry for Primary Industries.

Meinshausen, M., Smith, S. J., Calvin, K., Daniel, J. S., Kainuma, M., Lamarque, J.-F., . . Riahi, K. (2011). The RCP greenhouse gas concentrations and their extensions from 1765 to 2300 . Climatic change, 109(1-2), 213.

Merow, C., Smith, M. J., \& Silander, J. A., Jr. (2013). A practical guide to MaxEnt for modeling species' distributions: what it does, and why inputs and settings matter. Ecography, 36(10), 10581069. doi:10.1111/j.1600-0587.2013.07872.x

Mestre, F., Pita, R., Paupério, J., Martins, F., Alves, P., Mira, A., \& Beja, P. (2015). Combining distribution modelling and non-invasive genetics to improve range shift forecasting (Vol. 297).

McCallum. K. P, Guerin G. R., Breed, M. F., Lowe, A. J. (2014). Combining population genetics, species distribution modelling and field assessments to understand a species vulnerability to climate change. Austral Ecology, 39(1), 17-28. doi:doi:10.1111/aec.12041

Miller, J. A., \& Holloway, P. (2015). Incorporating movement in species distribution models. Progress in Physical Geography: Earth and Environment, 39(6), 837-849. doi:10.1177/0309133315580890

Miller, K. A., \& Munro, G. R. (2004). Climate and cooperation: a new perspective on the management of shared fish stocks. Marine Resource Economics, 19(3), 367-393.

Ministry for Primary Industries (2013). Fisheries Assessment Plenary, May 2013: stock assessments and yield estimates.

Ministry for the Environment (2018). Climate Change Projections for New Zealand: Atmosphere Projections Based on Simulations from the IPCC Fifth Assessment, 2nd Edition. Wellington: Ministry for the Environment.

Moore, C., Drazen, J. C., Radford, B. T., Kelley, C., \& Newman, S. J. (2016). Improving essential fish habitat designation to support sustainable ecosystem-based fisheries management. Marine Policy, 69, 32-41.

Moore, J. K., Fu, W., Primeau, F., Britten, G. L., Lindsay, K., Long, M., ... Randerson, J. T. (2018). Sustained climate warming drives declining marine biological productivity. Science, 359(6380), 1139-1143. doi:10.1126/science.aao6379

Morales, N. S., Fernandez, I. C., \& Baca-Gonzalez, V. (2017). MaxEnt's parameter configuration and small samples: are we paying attention to recommendations? A systematic review. Peerj, 5. doi:10.7717/peerj.3093

Morley, J. W., Selden, R. L., Latour, R. J., Frölicher, T. L., Seagraves, R. J., \& Pinsky, M. L. (2018). Projecting shifts in thermal habitat for 686 species on the North American continental shelf. Plos One, 13(5), e0196127. 
Morrison, M., Stevenson, M. L., \& Hanchet, S. M. (2001). Review of Bay of Plenty trawl survey time series, 1983-99 (Vol. 107): NIWA.

Mundy, B. C. (2005). Checklist of the fishes of the Hawaiian Archipelago. Bishop Mus. Bull. Zool., 6, 1704.

Muscarella, R., Galante, P. J., Soley-Guardia, M., Boria, R. A., Kass, J. M., Uriarte, M., \& Anderson, R. P. (2014). ENMeval: An R package for conducting spatially independent evaluations and estimating optimal model complexity for Maxent ecological niche models. Methods in Ecology and Evolution, 5(11), 1198-1205.

Neilson, M. (2019). New Zealand seas in 2018 hottest since records began, dire warning for marine life.

Nelder, J. A., \& Wedderburn, R. W. (1972). Generalized linear models. Journal of the Royal Statistical Society: Series A (General), 135(3), 370-384.

Nogués-Bravo, D., Rodríguez-Sánchez, F., Orsini, L., De Boer, E., Jansson, R., Morlon, H., . . Jackson, S. T. (2018). Cracking the code of biodiversity responses to past climate change. Trends in ecology \& evolution.

O'Driscoll, R. L., Dunford, A. J., \& Dunn, A. (2016). Industry acoustic surveys of spawning southern blue whiting on the Bounty Platform, New Zealand. Fisheries Research, 178, 61-70. doi:10.1016/j.fishres.2015.05.007

O'Driscoll, R., MacGibbon, D., Fu, S., Lyon, W., \& Stevens, D. (2011). A review of hoki and middledepth trawl surveys of the Chatham Rise, January 1992-2010. New Zealand Fisheries Assessment Report, 47, 814.

Papeş, M., \& Gaubert, P. (2007). Modelling ecological niches from low numbers of occurrences: assessment of the conservation status of poorly known viverrids (Mammalia, Carnivora) across two continents. Diversity and Distributions, 13(6), 890-902.

Paredes, F., \& Bravo, R. (2005). Reproductive cycle, size at first maturation and fecundity in the golden ling, Genypterus blacodes, in Chile. New Zealand Journal of Marine and Freshwater Research, 39(5), 1085-1096. doi:10.1080/00288330.2005.9517377

Parra, J. L., Graham, C. C., \& Freile, J. F. (2004). Evaluating alternative data sets for ecological niche models of birds in the Andes. Ecography, 27(3), 350-360.

Parsons, D., Sim-Smith, C., Cryer, M., Francis, M., Hartill, B., Jones, E., ... Morrison, M. (2014). Snapper (Chrysophrys auratus): a review of life history and key vulnerabilities in New Zealand. New Zealand Journal of Marine and Freshwater Research, 48(2), 256-283.

Paul, L. J. (1986). New Zealand Fisheries. Auckland.

Pearson, R. G., Raxworthy, C. J., Nakamura, M., \& Peterson, A. T. (2007). Predicting species distributions from small numbers of occurrence records: a test case using cryptic geckos in Madagascar. Journal of Biogeography, 34(1), 102-117. doi:10.1111/j.13652699.2006.01594.x

Perry, A. L., Low, P. J., Ellis, J. R., \& Reynolds, J. D. (2005). Climate change and distribution shifts in marine fishes. Science, 308(5730), 1912-1915.

Peterson, A. T. (2001). Predicting species' geographic distributions based on ecological niche modeling. The Condor, 103(3), 599-605.

Peterson, A. T. (2006). Uses and requirements of ecological niche models and related distributional models.

Peterson, A. T., Papeş, M., \& Eaton, M. (2007). Transferability and model evaluation in ecological niche modeling: a comparison of GARP and Maxent. Ecography, 30(4), 550-560.

Peterson, A. T., Papes, M., \& Soberon, J. (2008). Rethinking receiver operating characteristic analysis applications in ecological niche modeling. Ecological Modelling, 213(1), 63-72.

doi:10.1016/j.ecolmodel.2007.11.008 
Peterson, A. T., Sober, xf, n, J., Pearson, R. G., Anderson, R. P., ... jo, M. B. (2011). Ecological Niches and Geographic Distributions (MPB-49): Princeton University Press.

Phillips, B. (2008). Lobsters: biology, management, aquaculture and fisheries: John Wiley \& Sons.

Phillips, S. (2006). A brief tutorial on Maxent. AT\&T Research. In.

Phillips, S. J. (2008). Transferability, sample selection bias and background data in presence-only modelling: a response to Peterson et al.(2007). Ecography, 31(2), 272-278.

Phillips, S. J., Anderson, R. P., \& Schapire, R. E. (2006). Maximum entropy modeling of species geographic distributions. Ecological Modelling, 190(3-4), 231-259. doi:10.1016/j.ecolmodel.2005.03.026

Phillips, S. J., \& Dudík, M. (2008). Modeling of species distributions with Maxent: new extensions and a comprehensive evaluation. Ecography, 31(2), 161-175.

Phillips, S. J., Dudík, M., Elith, J., Graham, C. H., Lehmann, A., Leathwick, J., \& Ferrier, S. (2009). Sample selection bias and presence-only distribution models: implications for background and pseudo-absence data. Ecological Applications, 19(1), 181-197.

Phillips, S. J., Dudík, M., \& Schapire, R. E. (2004). A maximum entropy approach to species distribution modeling. Paper presented at the Proceedings of the twenty-first international conference on Machine learning.

Pinkerton, M., Rickard, G., Nodder, S., \& Macdonald, H. (2016). Climate change impacts and implications: net primary production, particulate flux and impacts on fish species in the New Zealand region. Retrieved from

Plagányi, É. (2019). Climate change impacts on fisheries. Science, 363(6430), 930-931. doi:10.1126/science.aaw5824

Planque, B., Bellier, E., \& Loots, C. (2011). Uncertainties in projecting spatial distributions of marine populations. Ices Journal of Marine Science, 68(6), 1045-1050. doi:10.1093/icesjms/fsr007

Poloczanska, E. S., Brown, C. J., Sydeman, W. J., Kiessling, W., Schoeman, D. S., Moore, P. J., . . . Burrows, M. T. (2013). Global imprint of climate change on marine life. Nature Climate Change, 3(10), 919.

Ponder, W. F., Carter, G. A., Flemons, P., \& Chapman, R. R. (2001). Evaluation of Museum Collection Data for Use in Biodiversity Assessment. Conservation Biology, 15(3), 648-657. doi:doi:10.1046/j.1523-1739.2001.015003648.x

Radford, C. A., Putland, R. L., \& Mensinger, A. F. (2018). Barking mad: The vocalisation of the john dory, Zeus faber. Plos One, 13(10), e0204647.

Radosavljevic, A., \& Anderson, R. P. (2014). Making better Maxent models of species distributions: complexity, overfitting and evaluation. Journal of Biogeography, 41(4), 629-643.

Ray, D., Behera, M. D., \& Jacob, J. (2018). Evaluating ecological niche models: A comparison between Maxent and GARP for predicting distribution of Hevea brasiliensis in India. Proceedings of the National Academy of Sciences, India Section B: Biological Sciences, 88(4), 1337-1343.

Reddy, S., \& Dávalos, L. M. (2003). Geographical sampling bias and its implications for conservation priorities in Africa. Journal of Biogeography, 30(11), 1719-1727.

Reznick, D. N., \& Ghalambor, C. K. (2001). The population ecology of contemporary adaptations: what empirical studies reveal about the conditions that promote adaptive evolution. Genetica, 112, 183-198.

Roberts, C., Stewart, A. L., \& Struthers, C. D. (2015). The Fishes of New Zealand: Te Papa Press.

Roberts, D. R., Bahn, V., Ciuti, S., Boyce, M. S., Elith, J., Guillera-Arroita, G., ... Thuiller, W. (2017). Cross-validation strategies for data with temporal, spatial, hierarchical, or phylogenetic structure. Ecography, 40(8), 913-929. 
Robinson, L., Elith, J., Hobday, A., Pearson, R., Kendall, B., Possingham, H., \& Richardson, A. (2011). Pushing the limits in marine species distribution modelling: lessons from the land present challenges and opportunities. Global Ecology and Biogeography, 20(6), 789-802.

Robinson, L., Hobday, A., Possingham, H., \& Richardson, A. J. (2015). Trailing edges projected to move faster than leading edges for large pelagic fish habitats under climate change. Deep Sea Research Part II: Topical Studies in Oceanography, 113, 225-234.

Robinson, N. M., Nelson, W. A., Costello, M. J., Sutherland, J. E., \& Lundquist, C. J. (2017). A Systematic Review of Marine-Based Species Distribution Models (SDMs) with Recommendations for Best Practice. Frontiers in Marine Science, 4(421). doi:10.3389/fmars.2017.00421

Roper, C. F. E., Nigmatullin, C., \& Jereb, P. (2010). Family Ommastrephidae (Vol. 2).

Rutterford, L. A., Simpson, S. D., Jennings, S., Johnson, M. P., Blanchard, J. L., Schön, P.-J., . . Genner, M. J. (2015). Future fish distributions constrained by depth in warming seas. Nature Climate Change, 5(6), 569.

Sbrocco, E. J. (2014). Paleo-MARSPEC: Gridded ocean climate layers for the mid-Holocene and Last Glacial Maximum. Ecology, 95(6), 1710-1710.

Scales, K. L., Hazen, E. L., Jacox, M. G., Edwards, C. A., Boustany, A. M., Oliver, M. J., \& Bograd, S. J. (2017). Scale of inference: on the sensitivity of habitat models for wide-ranging marine predators to the resolution of environmental data. Ecography, 40(1), 210-220.

Schellnhuber, H. J., Rahmstorf, S., \& Winkelmann, R. (2016). Why the right climate target was agreed in Paris. Nature Climate Change, 6(7), 649.

Segurado, P., \& Araujo, M. B. (2004). An evaluation of methods for modelling species distributions. Journal of Biogeography, 31(10), 1555-1568.

Shabani, F., Kumar, L., \& Ahmadi, M. (2016). A comparison of absolute performance of different correlative and mechanistic species distribution models in an independent area. Ecology and evolution, 6(16), 5973-5986.

Shcheglovitova, M., \& Anderson, R. P. (2013). Estimating optimal complexity for ecological niche models: a jackknife approach for species with small sample sizes. Ecological Modelling, 269, 9-17.

Smith, P. J., Francis, R., \& Paul, L. J. (1978). GENETIC-VARIATION AND POPULATION-STRUCTURE IN THE NEW-ZEALAND SNAPPER. New Zealand Journal of Marine and Freshwater Research, 12(4), 343-350. doi:10.1080/00288330.1978.9515761

Smyth, K., \& Elliott, M. (2016). Effects of changing salinity on the ecology of the marine environment. In.

Song, W., Kim, E., Lee, D., Lee, M., \& Jeon, S.-W. (2013). The sensitivity of species distribution modeling to scale differences. Ecological Modelling, 248, 113-118.

Steffen, W., Rockström, J., Richardson, K., Lenton, T. M., Folke, C., Liverman, D., ... Schellnhuber, H. J. (2018). Trajectories of the Earth System in the Anthropocene. Proceedings of the National Academy of Sciences, 115(33), 8252-8259. doi:10.1073/pnas.1810141115

Stevens. (2014). Trawl survey of hoki and middle-depth species on the Chatham Rise, January 2014 (TAN1401) New Zealand Fisheries Assessment Report 2015/19

Stevens, C., \& Noll, B. (2019). Coastal seas around New Zealand are heading into a marine heatwave, again. Retrieved from http://theconversation.com/coastal-seas-around-new-zealand-areheading-into-a-marine-heatwave-again-

Stevenson, M., \& MacGibbon, D. (2018). Inshore trawl survey of the west coast South Island and Tasman and Golden Bays, March-April 2017 (KAH1703). New Zealand Fisheries Assessment Report, 92. 
Sullivan, M. J. P., Davies, R. G., Reino, L., \& Franco, A. M. A. (2012). Using dispersal information to model the species-environment relationship of spreading non-native species. Methods in Ecology and Evolution, 3(5), 870-879. doi:10.1111/j.2041-210X.2012.00219.x

Sumpton, W. D., Ovenden, J. R., Keenan, C. P., \& Street, R. (2008). Evidence for a stock discontinuity of snapper (Pagrus auratus) on the East coast of Australia. Fisheries Research, 94(1), 92-98. doi:10.1016/j.fishres.2008.07.001

Sunday, J. M., Bates, A. E., \& Dulvy, N. K. (2012). Thermal tolerance and the global redistribution of animals. Nature Climate Change, 2(9), 686.

Syfert, M. M., Smith, M. J., \& Coomes, D. A. (2013). The effects of sampling bias and model complexity on the predictive performance of MaxEnt species distribution models. Plos One, $8(2)$, e55158.

Tarkesh, M., \& Jetschke, G. (2012). Comparison of six correlative models in predictive vegetation mapping on a local scale. Environmental and Ecological Statistics, 19(3), 437-457. doi:10.1007/s10651-012-0194-3

Terribile, L. C., \& Diniz-Filho, J. A. F. (2010). How many studies are necessary to compare niche-based models for geographic distributions? Inductive reasoning may fail at the end. Brazilian Journal of Biology, 70(2), 263-269.

Thuiller, W., Richardson, D. M., PYŠEK, P., Midgley, G. F., Hughes, G. O., \& Rouget, M. (2005). Nichebased modelling as a tool for predicting the risk of alien plant invasions at a global scale. Global Change Biology, 11(12), 2234-2250.

Tingley, G., \& Dunn, M. E. (2018). Global review of orange roughy (Hoplostethus atlanticus), their fisheries, biology and management. FAO Fisheries and Aquaculture technical paper No. 622. Rome. Licence CC BY-NC-SA 3.0 IGO.

Torres, L. G., Smith, T. D., Sutton, P., MacDiarmid, A., Bannister, J., \& Miyashita, T. (2013). From exploitation to conservation: habitat models using whaling data predict distribution patterns and threat exposure of an endangered whale. Diversity and Distributions, 19(9), 1138-1152.

Tshudy, D. (2003). Clawed lobster (Nephropidae) diversity through time. Journal of Crustacean Biology, 23(1), 178-186.

Tuck, I. D., Parsons, D. M., Hartill, B. W., \& Chiswell, S. M. (2015). Scampi (Metanephrops challengeri) emergence patterns and catchability. Ices Journal of Marine Science, 72(suppl_1), i199-i210.

Tyberghein, L., Verbruggen, H., Pauly, K., Troupin, C., Mineur, F., \& De Clerck, O. (2012). Bio-ORACLE: a global environmental dataset for marine species distribution modelling. Global Ecology and Biogeography, 21(2), 272-281.

Uozumi, Y. (1998). Fishery biology of arrow squids, Nototodarus gouldi and N. sloanii, in New Zealand waters. Bulletin of the National Research Institute of Far Seas Fisheries, 35, 1-111.

van der Reis, A. L., Laroche, O., Jeffs, A. G., \& Lavery, S. D. (2018). Preliminary analysis of New Zealand scampi (Metanephrops challengeri) diet using metabarcoding. Peerj, 6, e5641.

Van Vuuren, D. P., Edmonds, J., Kainuma, M., Riahi, K., Thomson, A., Hibbard, K., . . Lamarque, J.-F. (2011). The representative concentration pathways: an overview. Climatic change, 109(1-2), 5.

VanDerWal, J., Shoo, L. P., Graham, C., \& Williams, S. E. (2009). Selecting pseudo-absence data for presence-only distribution modeling: how far should you stray from what you know? Ecological Modelling, 220(4), 589-594.

Varela, A. I., Ritchie, P. A., \& Smith, P. J. (2013). Global genetic population structure in the commercially exploited deep-sea teleost orange roughy (Hoplostethus atlanticus) based on microsatellite DNA analyses. Fisheries Research, 140, 83-90.

doi:10.1016/j.fishres.2012.12.011

Veloz, S. D. (2009). Spatially autocorrelated sampling falsely inflates measures of accuracy for presence-only niche models. Journal of Biogeography, 36(12), 2290-2299. 
Warren, D. L., Glor, R. E., \& Turelli, M. (2010). ENMTools: a toolbox for comparative studies of environmental niche models. Ecography, 33(3), 607-611. doi:doi:10.1111/j.16000587.2009.06142.x

Warren, D. L., \& Seifert, S. N. (2011). Ecological niche modeling in Maxent: the importance of model complexity and the performance of model selection criteria. Ecological Applications, 21(2), 335-342.

Weinert, M., Mathis, M., Kröncke, I., Neumann, H., Pohlmann, T., \& Reiss, H. (2016). Modelling climate change effects on benthos: Distributional shifts in the North Sea from 2001 to 2099. Estuarine, Coastal and Shelf Science, 175, 157-168.

Wenger, S. J. \& Olden J. D. Assessing transferability of ecological models: an underappreciated aspect of statistical validation. Methods in Ecology and Evolution, 3(2), 260-267. doi:doi:10.1111/j.2041-210X.2011.00170.x

Werkowska, W., Marquez, A. L., Real, R., \& Acevedo, P. (2017). A practical overview of transferability in species distribution modeling. Environmental Reviews, 25(1), 127-133. doi:10.1139/er2016-0045

Yates, C. J., McNeill, A., Elith, J., \& Midgley, G. F. (2010). Assessing the impacts of climate change and land transformation on Banksia in the South West Australian Floristic Region. Diversity and Distributions, 16(1), 187-201.

Zaniewski, A. E., Lehmann, A., \& Overton, J. M. (2002). Predicting species spatial distributions using presence-only data: a case study of native New Zealand ferns. Ecological Modelling, 157(23), 261-280.

Zeng, Y., Low, B. W., \& Yeo, D. C. (2016). Novel methods to select environmental variables in MaxEnt: A case study using invasive crayfish. Ecological Modelling, 341, 5-13.

Zhang, X., \& Vincent, A. C. J. (2017). Integrating multiple datasets with species distribution models to inform conservation of the poorly-recorded Chinese seahorses. Biological Conservation, 211, 161-171. doi:10.1016/j.biocon.2017.05.020

Zurell, D. (2017). Integrating demography, dispersal and interspecific interactions into bird distribution models. Journal of Avian Biology, 48(12), 1505-1516. doi:10.1111/jav.01225

Zuur, A. F., leno, E. N., \& Elphick, C. S. (2010). A protocol for data exploration to avoid common statistical problems. Methods in Ecology and Evolution, 1(1), 3-14. 


\section{Appendix}

Appendix A: Additional information about the trawl survey data obtained from NIWA (M. Dunn)

Appendix A.1: Number of records per year

\begin{tabular}{|c|c|}
\hline year & No. \\
\hline 1960 & 838 \\
\hline 1961 & 1187 \\
\hline 1962 & 1298 \\
\hline 1963 & 518 \\
\hline 1964 & 388 \\
\hline 1965 & 603 \\
\hline 1968 & 262 \\
\hline 1969 & 925 \\
\hline 1970 & 139 \\
\hline 1971 & 741 \\
\hline 1972 & 713 \\
\hline 1973 & 887 \\
\hline 1974 & 383 \\
\hline 1975 & 423 \\
\hline 1976 & 849 \\
\hline 1977 & 896 \\
\hline 1978 & 1604 \\
\hline 1979 & 16981 \\
\hline 1980 & 6711 \\
\hline 1981 & 16607 \\
\hline 1982 & 18948 \\
\hline 1983 & 19752 \\
\hline 1984 & 14951 \\
\hline 1985 & 14859 \\
\hline 1986 & 21961 \\
\hline 1987 & 14042 \\
\hline 1988 & 12553 \\
\hline 1989 & 23140 \\
\hline 1990 & 30642 \\
\hline 1991 & 10436 \\
\hline 1992 & 31422 \\
\hline 1993 & 24456 \\
\hline 1994 & 27889 \\
\hline 1995 & 24472 \\
\hline 1996 & 19602 \\
\hline 1997 & 9968 \\
\hline 1998 & 11222 \\
\hline 1999 & 11204 \\
\hline 2000 & 17977 \\
\hline 2001 & 13998 \\
\hline 2002 & 18005 \\
\hline 2003 & 8509 \\
\hline 2004 & 12127 \\
\hline 2005 & 15659 \\
\hline 2006 & 8084 \\
\hline
\end{tabular}




\begin{tabular}{ll}
\hline 2007 & 19889 \\
2008 & 22371 \\
2009 & 13861 \\
2010 & 20215 \\
2011 & 12298 \\
2012 & 18984 \\
2013 & 14059 \\
2014 & 12664 \\
2015 & 9306 \\
2016 & 20609 \\
2017 & 7818 \\
total & 660905 \\
\hline
\end{tabular}

Appendix A.2: Summary statistics of catch records

\begin{tabular}{|c|c|c|c|c|c|c|c|c|c|c|c|}
\hline trip_code & $\begin{array}{l}\text { station_n } \\
\text { o }\end{array}$ & species & weight & wt_meth & oth_data & longitude & latitude & $\begin{array}{l}\min \_ \text {gdep } \\
\text { th }\end{array}$ & $\begin{array}{l}\text { max_gd } \\
\text { epth }\end{array}$ & $\begin{array}{l}\text { gear_met } \\
\text { h }\end{array}$ & $\begin{array}{l}\text { gear_per } \\
f\end{array}$ \\
\hline $\begin{array}{l}\tan 9406: \\
6369\end{array}$ & $\begin{array}{l}\text { Min. : } \\
1.00\end{array}$ & $\begin{array}{l}\text { HOK : } \\
14250\end{array}$ & $\begin{array}{l}\text { Min. : } \\
0.0\end{array}$ & $\begin{array}{l}\text { Min. } \\
: 1.00\end{array}$ & $\begin{array}{l}000 \\
: 212328\end{array}$ & $\begin{array}{l}\text { Min. : } \\
53.34\end{array}$ & $\begin{array}{l}\text { Min. :- } \\
76.83\end{array}$ & $\begin{array}{l}\text { Min. : } \\
2.0\end{array}$ & $\begin{array}{l}\text { Min. : } \\
4\end{array}$ & $\begin{array}{l}\text { Min. } \\
: 1.000\end{array}$ & $\begin{array}{l}\text { Min. } \\
: 1.00\end{array}$ \\
\hline $\begin{array}{l}\text { cor9002: } \\
5628\end{array}$ & $\begin{array}{l}\text { 1st Qu.: } \\
24.00\end{array}$ & $\begin{array}{l}\text { LIN : } \\
10967\end{array}$ & $\begin{array}{l}\text { 1st Qu.: } \\
0.8\end{array}$ & $\begin{array}{l}\text { 1st } \\
\text { Qu.:1.00 }\end{array}$ & :136039 & $\begin{array}{l}\text { 1st } \\
\text { Qu.:171.0 } \\
2\end{array}$ & $\begin{array}{l}\text { 1st Qu.:- } \\
44.49\end{array}$ & $\begin{array}{l}\text { 1st Qu.: } \\
150.0\end{array}$ & $\begin{array}{l}\text { 1st Qu.: } \\
159\end{array}$ & $\begin{array}{l}\text { 1st } \\
\text { Qu.:1.000 }\end{array}$ & $\begin{array}{l}\text { 1st } \\
\text { Qu.:1.00 }\end{array}$ \\
\hline $\begin{array}{l}\tan 9206: \\
5416\end{array}$ & $\begin{array}{l}\text { Median : } \\
55.00\end{array}$ & $\begin{array}{l}\text { JAV : } \\
10703\end{array}$ & $\begin{array}{l}\text { Median : } \\
3.4\end{array}$ & $\begin{array}{l}\text { Median } \\
: 1.00\end{array}$ & $\begin{array}{l}110: \\
84293\end{array}$ & $\begin{array}{l}\text { Median } \\
: 174.52\end{array}$ & $\begin{array}{l}\text { Median :- } \\
43.08\end{array}$ & $\begin{array}{l}\text { Median : } \\
550.0\end{array}$ & $\begin{array}{l}\text { Median : } \\
568\end{array}$ & $\begin{array}{l}\text { Median } \\
: 1.000\end{array}$ & $\begin{array}{l}\text { Median } \\
: 1.00\end{array}$ \\
\hline $\begin{array}{l}\text { buc8701: } \\
4756\end{array}$ & $\begin{array}{l}\text { Mean : } \\
72.36\end{array}$ & $\begin{array}{l}\text { SPD : } \\
10019\end{array}$ & $\begin{array}{l}\text { Mean : } \\
103.1\end{array}$ & $\begin{array}{l}\text { Mean } \\
: 1.12\end{array}$ & $\begin{array}{l}100: \\
71241\end{array}$ & $\begin{array}{l}\text { Mean } \\
: 172.19\end{array}$ & $\begin{array}{l}\text { Mean :- } \\
41.67\end{array}$ & $\begin{array}{l}\text { Mean : } \\
555.5\end{array}$ & $\begin{array}{l}\text { Mean : } \\
579\end{array}$ & $\begin{array}{l}\text { Mean } \\
: 1.215\end{array}$ & $\begin{array}{l}\text { Mean } \\
: 1.24\end{array}$ \\
\hline $\begin{array}{l}\tan 1601: \\
4604\end{array}$ & $\begin{array}{l}\text { 3rd Qu.: } \\
96.00\end{array}$ & $\begin{array}{l}\text { ORH : } \\
9605\end{array}$ & $\begin{array}{l}\text { 3rd Qu.: } \\
15.0\end{array}$ & $\begin{array}{l}\text { 3rd } \\
\text { Qu.:1.00 }\end{array}$ & $\begin{array}{l}1 \quad: \\
17252\end{array}$ & $\begin{array}{l}\text { 3rd } \\
\text { Qu.:179.2 } \\
3\end{array}$ & $\begin{array}{l}\text { 3rd Qu.:- } \\
41.27\end{array}$ & $\begin{array}{l}\text { 3rd Qu.: } \\
879.0\end{array}$ & $\begin{array}{l}\text { 3rd Qu.: } \\
915\end{array}$ & $\begin{array}{l}\text { 3rd } \\
\text { Qu.:1.000 }\end{array}$ & $\begin{array}{l}\text { 3rd } \\
\text { Qu.:1.00 }\end{array}$ \\
\hline $\begin{array}{l}\tan 1003: \\
4463\end{array}$ & $\begin{array}{l}\text { Max. } \\
: 928.00\end{array}$ & $\begin{array}{l}\text { GSP : } \\
9178\end{array}$ & $\begin{array}{l}\text { Max. } \\
: 200020.8\end{array}$ & $\begin{array}{l}\text { Max. } \\
: 8.00\end{array}$ & $\begin{array}{l}111: \\
11799\end{array}$ & $\begin{array}{l}\text { Max. } \\
: 188.11\end{array}$ & $\begin{array}{l}\text { Max. : } \\
25.60\end{array}$ & $\begin{array}{l}\text { Max. } \\
: 2730.0\end{array}$ & $\begin{array}{l}\text { Max. } \\
: 2730\end{array}$ & $\begin{array}{l}\text { Max. } \\
: 3.000\end{array}$ & $\begin{array}{l}\text { Max. } \\
: 2.00\end{array}$ \\
\hline $\begin{array}{l}\text { (Other):51 } \\
2673\end{array}$ & NA & $\begin{array}{l}\text { (Other):47 } \\
9187\end{array}$ & $\begin{array}{l}\text { NA's } \\
: 27161\end{array}$ & $\begin{array}{l}\text { NA's } \\
: 142008\end{array}$ & $\begin{array}{l}\text { (Other): } \\
10957\end{array}$ & NA & NA & $\begin{array}{l}\text { NA's } \\
: 27927\end{array}$ & $\begin{array}{l}\text { NA's } \\
: 27819\end{array}$ & NA & NA \\
\hline
\end{tabular}

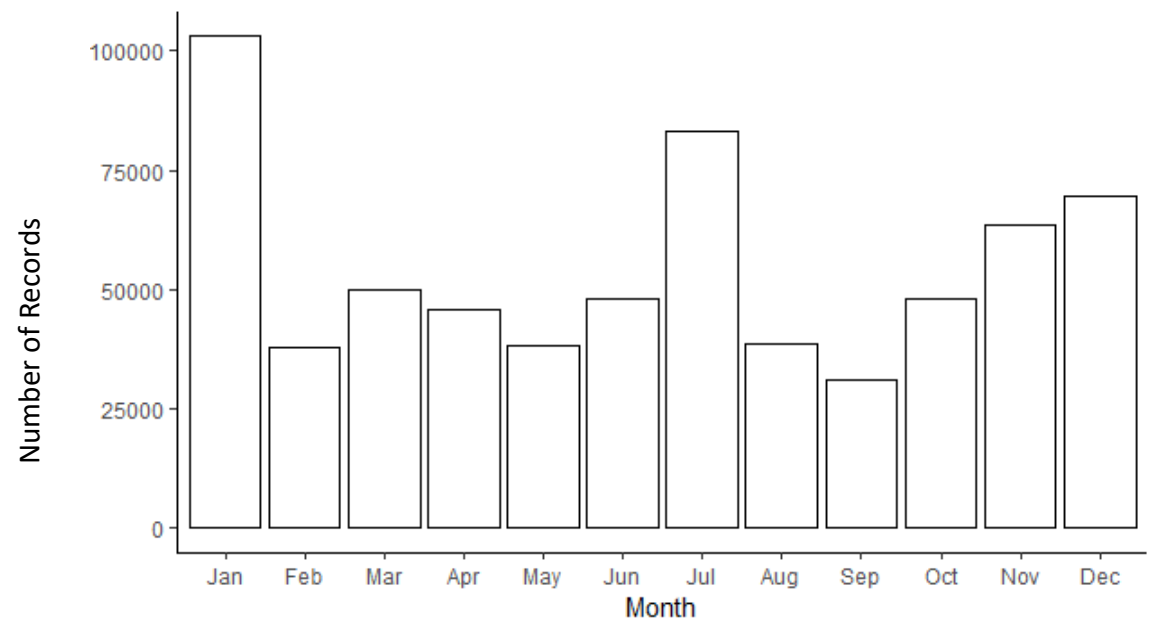

Appendix A.3: Months that surveys took place 


\section{Appendix B: Correlated covariates from Bio-ORACLE tests}

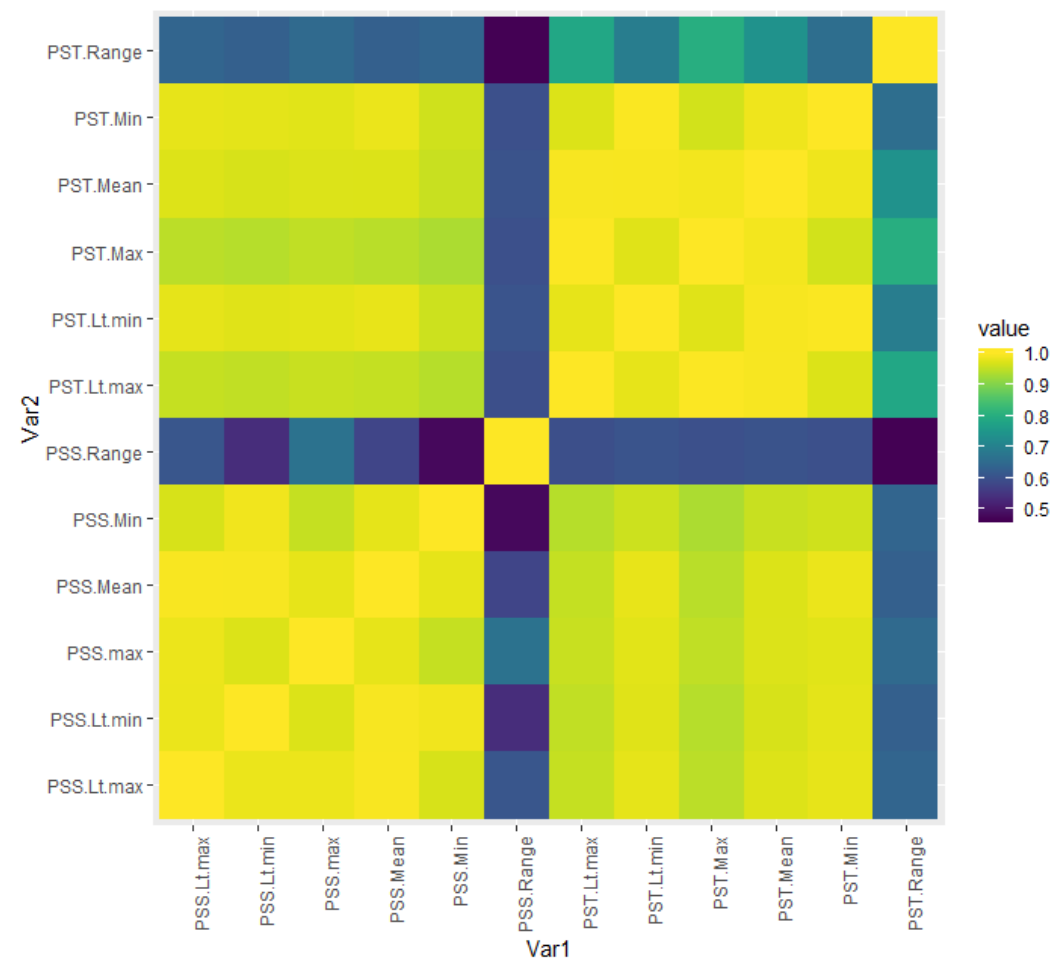

Appendix B.1: Correlation matrix between Bio-ORACLE temperature and salinity climate layers. (Spearman Correlations). 1 = highly correlated, 0 = no correlation.

Appendix B.2: Correlations between Bio-ORACLE temperature and salinity climate layers. (Spearman Correlations). 1 = highly correlated, $0=$ no correlation.

\begin{tabular}{|c|c|c|c|c|c|c|c|c|c|c|c|c|}
\hline & $\begin{array}{l}\text { PSS.Lt.m } \\
\text { ax }\end{array}$ & $\begin{array}{l}\text { PSS.Lt.m } \\
\text { in }\end{array}$ & $\begin{array}{l}\text { PSS.ma } \\
\mathrm{x}\end{array}$ & $\begin{array}{l}\text { PSS.Me } \\
\text { an }\end{array}$ & $\begin{array}{l}\text { PSS.Mi } \\
\mathrm{n}\end{array}$ & $\begin{array}{l}\text { PSS.Ran } \\
\text { ge }\end{array}$ & $\begin{array}{l}\text { PST.Lt.m } \\
\text { ax }\end{array}$ & $\begin{array}{l}\text { PST.Lt.m } \\
\text { in }\end{array}$ & $\begin{array}{l}\text { PST.Ma } \\
\mathrm{x}\end{array}$ & $\begin{array}{l}\text { PST.Me } \\
\text { an }\end{array}$ & $\begin{array}{l}\text { PST.Mi } \\
\mathrm{n}\end{array}$ & $\begin{array}{l}\text { PST.Ran } \\
\text { ge }\end{array}$ \\
\hline PSS.Lt.m & & & & & & & & & & & & \\
\hline$a x$ & 1 & 0.989 & 0.985 & 0.996 & 0.968 & 0.610 & 0.954 & 0.981 & 0.946 & 0.973 & 0.981 & 0.640 \\
\hline $\begin{array}{l}\text { PSS.Lt.m } \\
\text { in }\end{array}$ & & 1 & 0.972 & 0.995 & 0.990 & 0.536 & 0.951 & 0.976 & 0.942 & 0.968 & 0.979 & 0.629 \\
\hline $\begin{array}{l}\text { PSS.max } \\
\text { PSS.Mea }\end{array}$ & & & 1 & 0.981 & 0.954 & 0.668 & 0.957 & 0.977 & 0.950 & 0.972 & 0.976 & 0.650 \\
\hline $\mathrm{n}$ & & & & 1 & 0.980 & 0.577 & 0.953 & 0.982 & 0.945 & 0.972 & 0.985 & 0.630 \\
\hline $\begin{array}{l}\text { PSS.Min } \\
\text { PSS.Ran }\end{array}$ & & & & & 1 & 0.480 & 0.943 & 0.960 & 0.935 & 0.956 & 0.962 & 0.640 \\
\hline $\begin{array}{l}\text { ge } \\
\text { PST.Lt.m }\end{array}$ & & & & & & 1 & 0.595 & 0.608 & 0.598 & 0.605 & 0.598 & 0.469 \\
\hline $\begin{array}{l}\text { ax } \\
\text { PST.Lt.m }\end{array}$ & & & & & & & 1 & 0.986 & 0.998 & 0.995 & 0.972 & 0.785 \\
\hline in & & & & & & & & 1 & 0.976 & 0.994 & 0.997 & 0.693 \\
\hline $\begin{array}{l}\text { PST.Max } \\
\text { PST.Mea }\end{array}$ & & & & & & & & & 1 & 0.991 & 0.965 & 0.801 \\
\hline $\mathrm{n}$ & & & & & & & & & & 1 & 0.988 & 0.741 \\
\hline $\begin{array}{l}\text { PST.Min } \\
\text { PST.Ran }\end{array}$ & & & & & & & & & & & 1 & 0.658 \\
\hline ge & & & & & & & & & & & & 1 \\
\hline
\end{tabular}



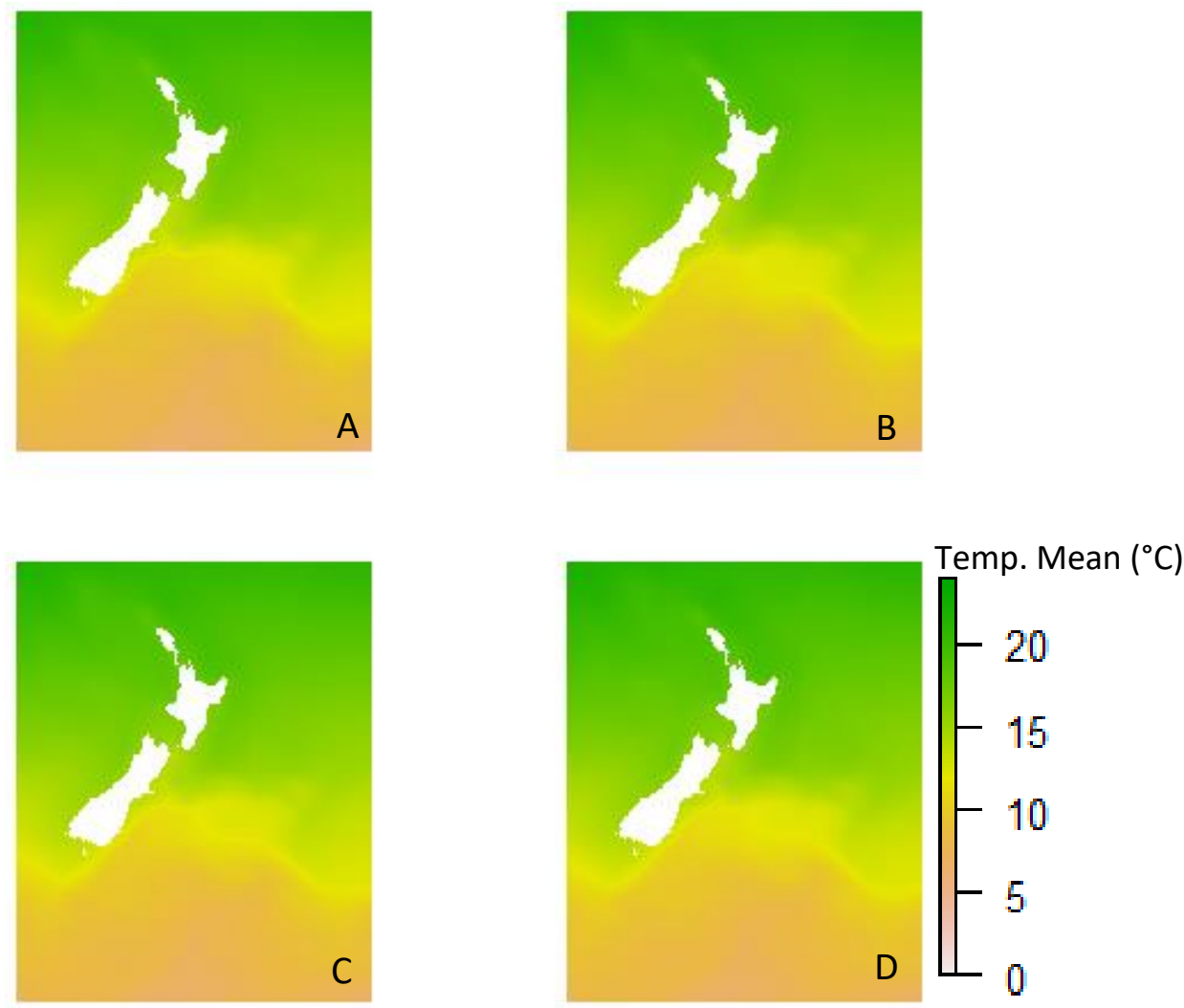

Appendix C.1: ST Mean 2050 climate layers under RCP scenarios 26(A), 4.5(B), 6.0(C) and 8.5(D).
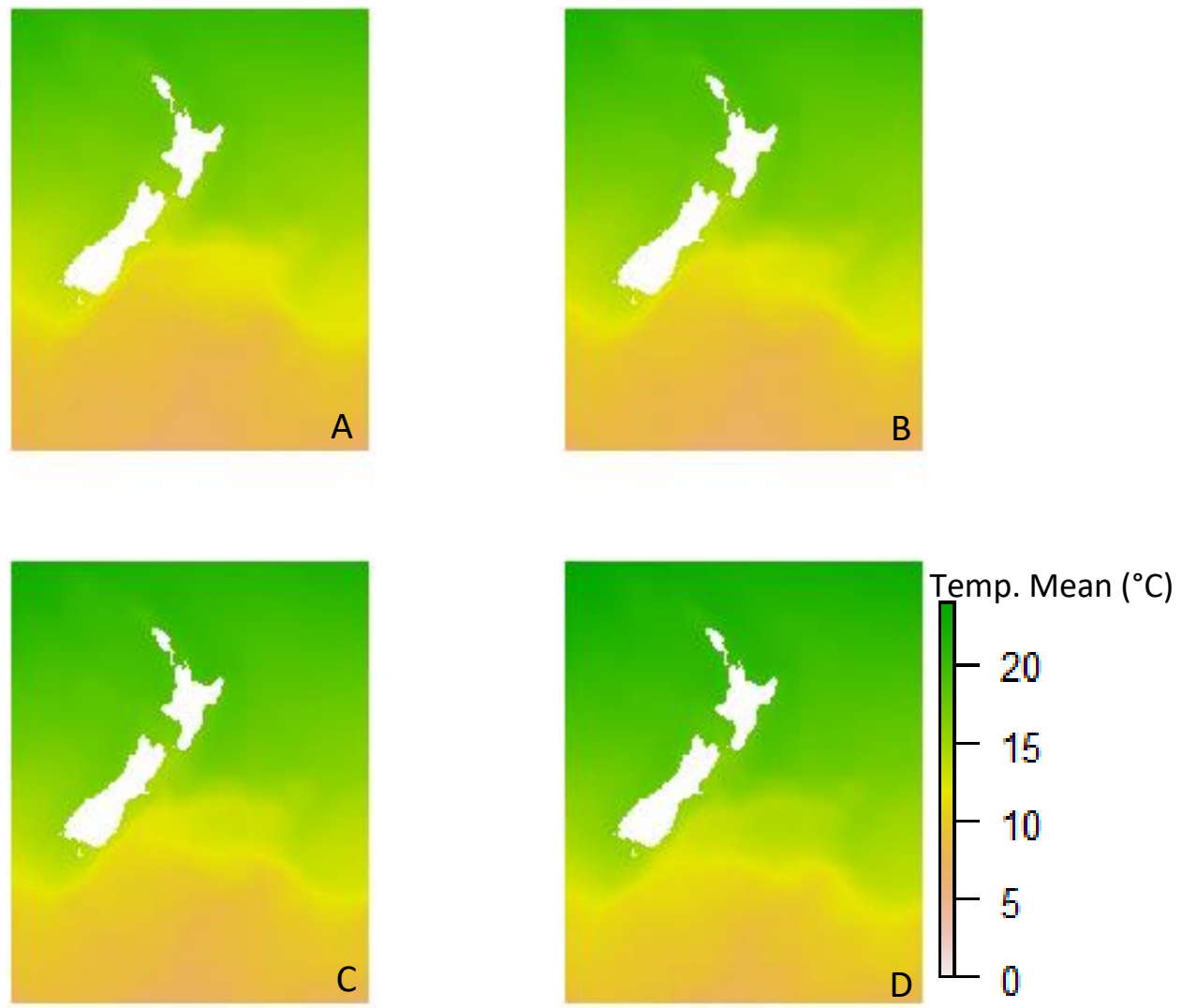

Appendix C.2: ST Mean 2100 climate layers under RCP scenarios 26(A), 4.5(B), 6.0(C) and 8.5(D). 

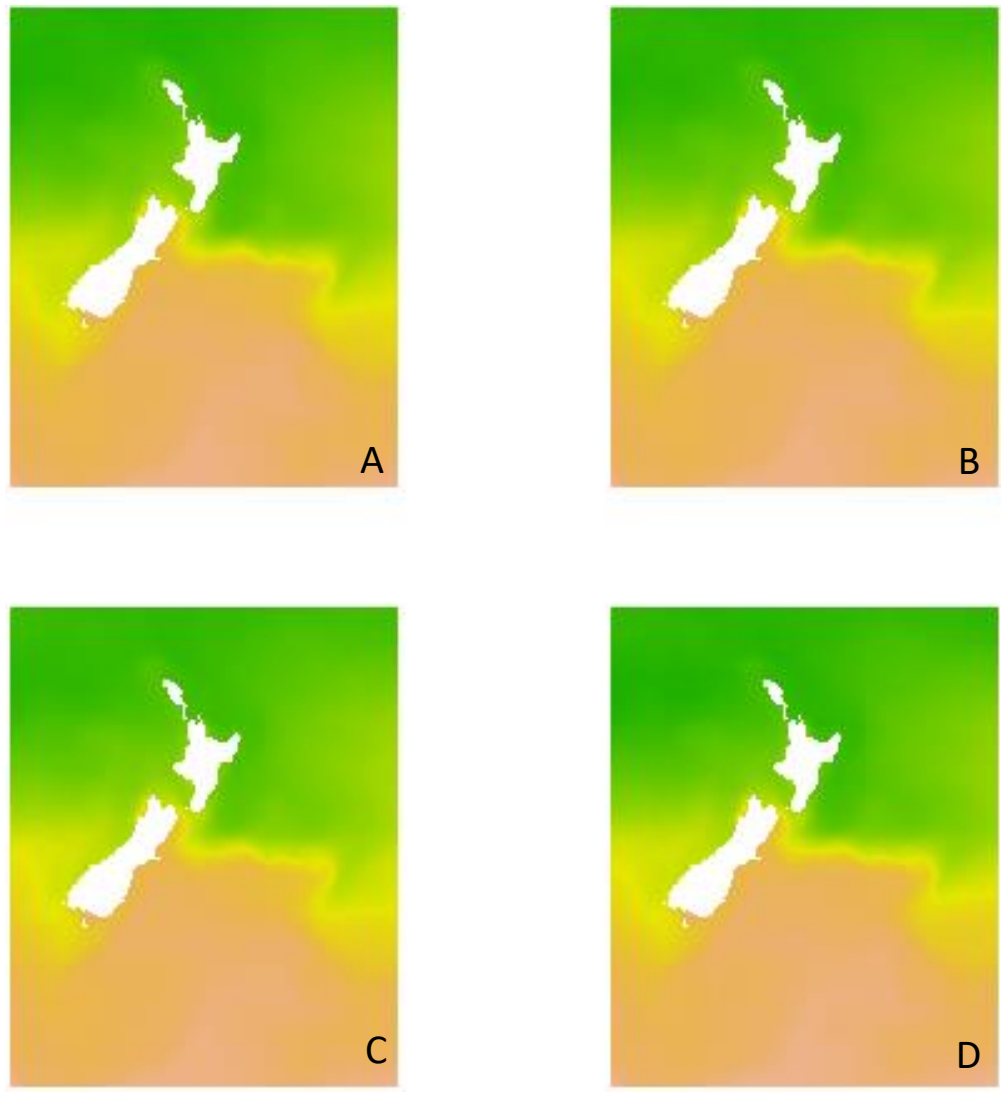

Salinity Mean (psu)

T 36.0

$-35.5$

- 35.0

$-34.5$

- 34.0

Appendix C.3: SS Mean 2050 climate layers under RCP scenarios 26(A), 4.5(B), 6.0(C) and 8.5(D).
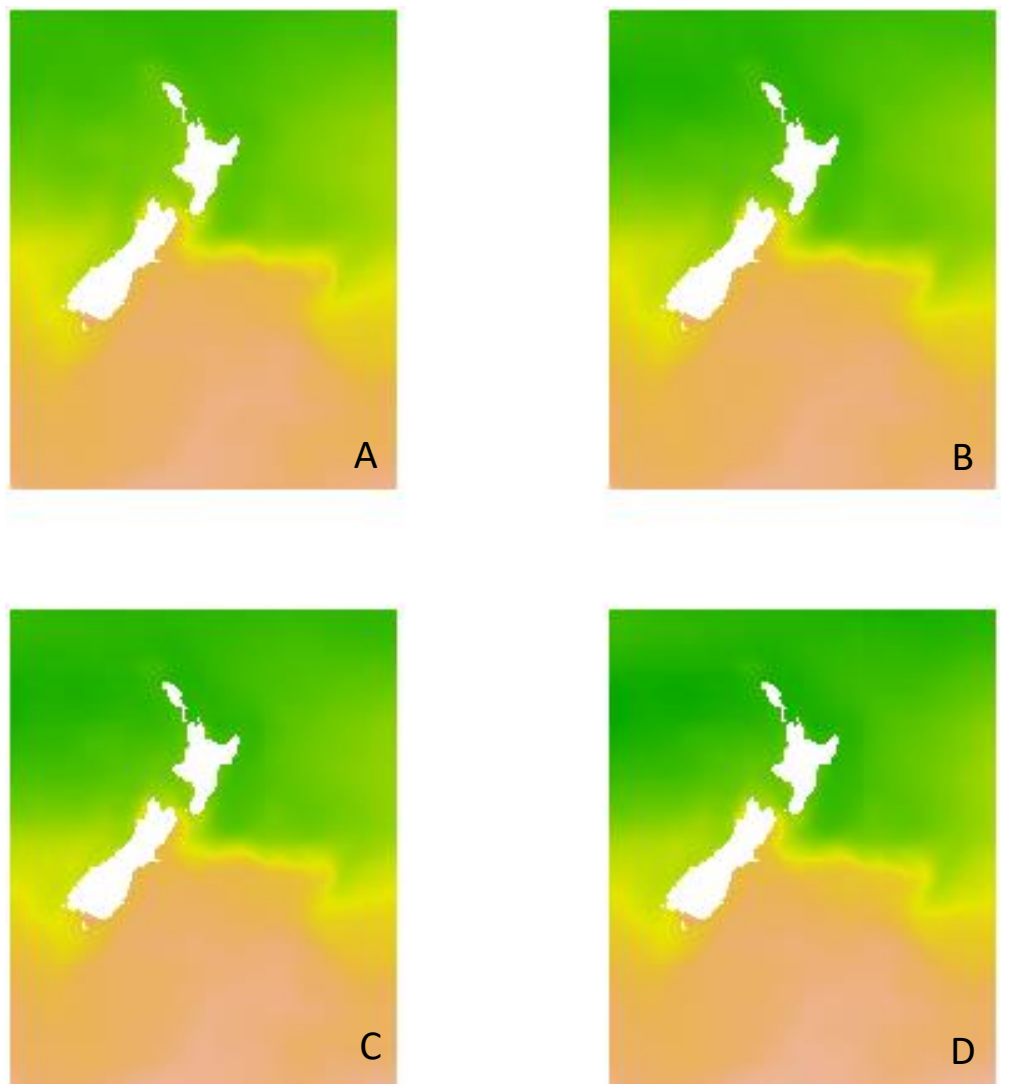

Salinity Mean (psu)

ए 36.0

35.5

$-35.0$

- 34.5

34.0

C

D

33.5

Appendix C.4: SS Mean 2100 climate layers under RCP scenarios 26(A), 4.5(B), 6.0(C) and 8.5(D). 

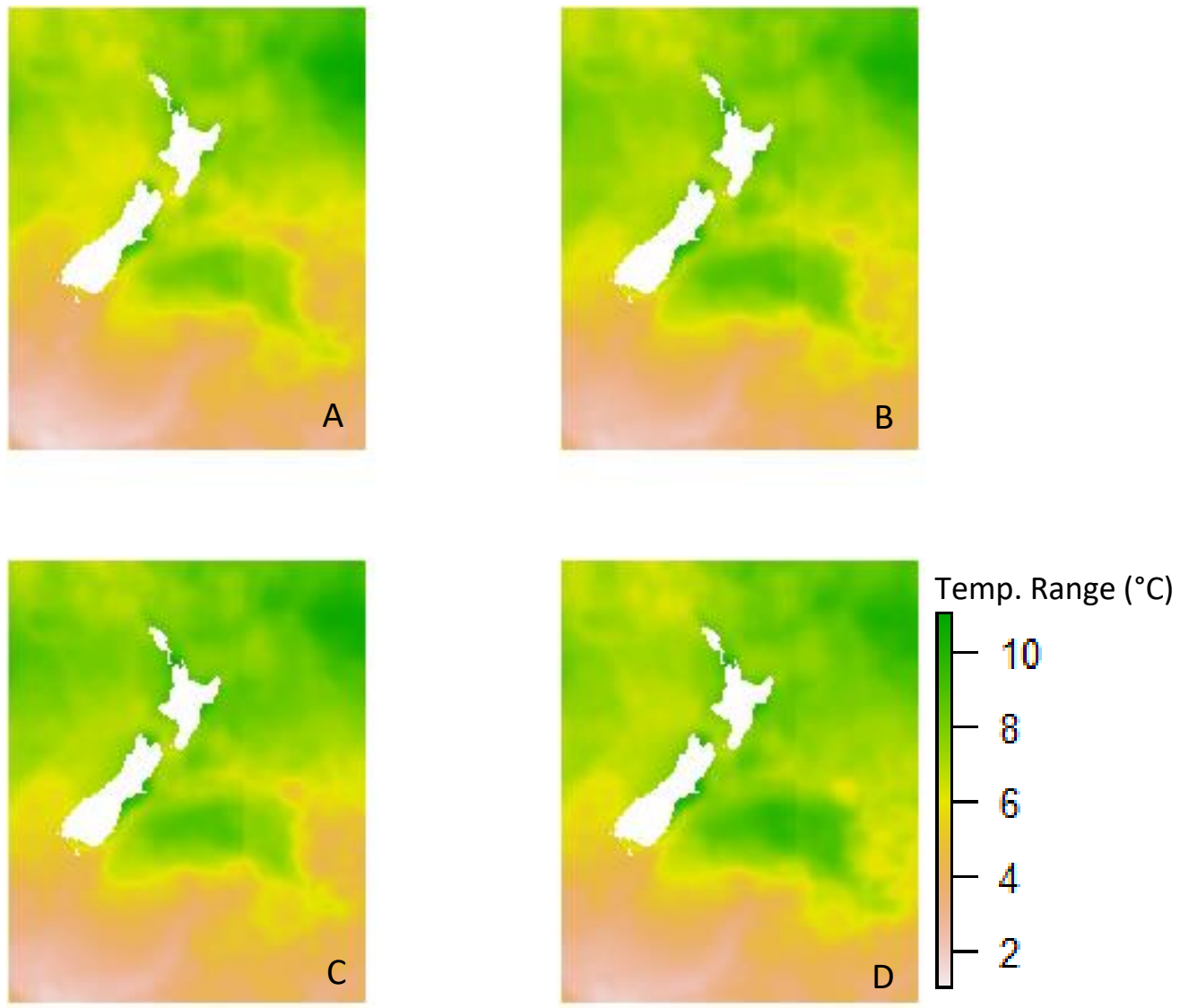

Appendix C.5: ST Range 2050 climate layers under RCP scenarios 26(A), 4.5(B), 6.0(C) and 8.5(D).
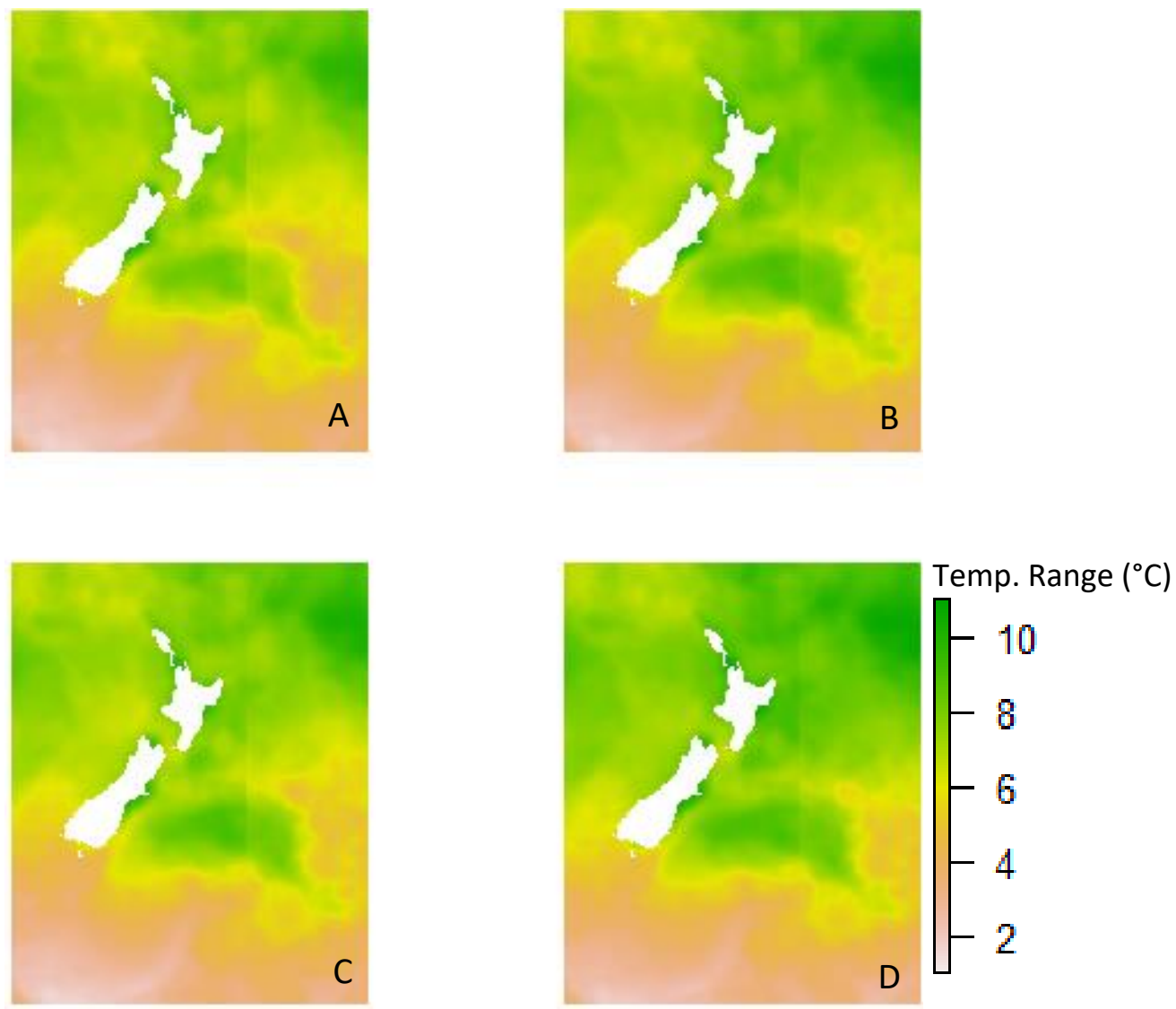

Appendix C.6: ST Range 2100 climate layers under RCP scenarios 26(A), 4.5(B), 6.0(C) and 8.5(D). 

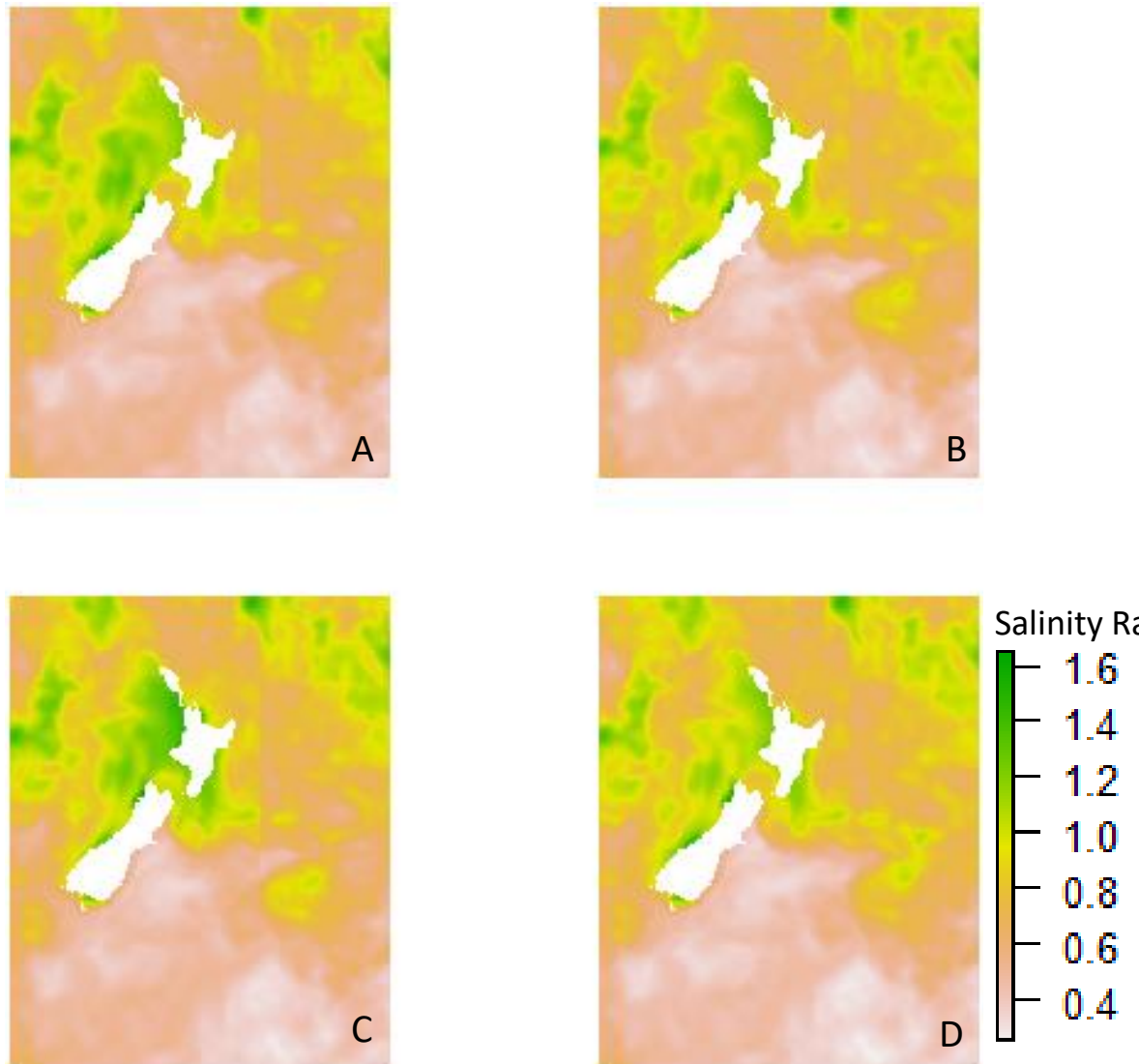

Appendix C.7: SS Range 2050 climate layers under RCP scenarios 26(A), 4.5(B), 6.0(C) and 8.5(D).
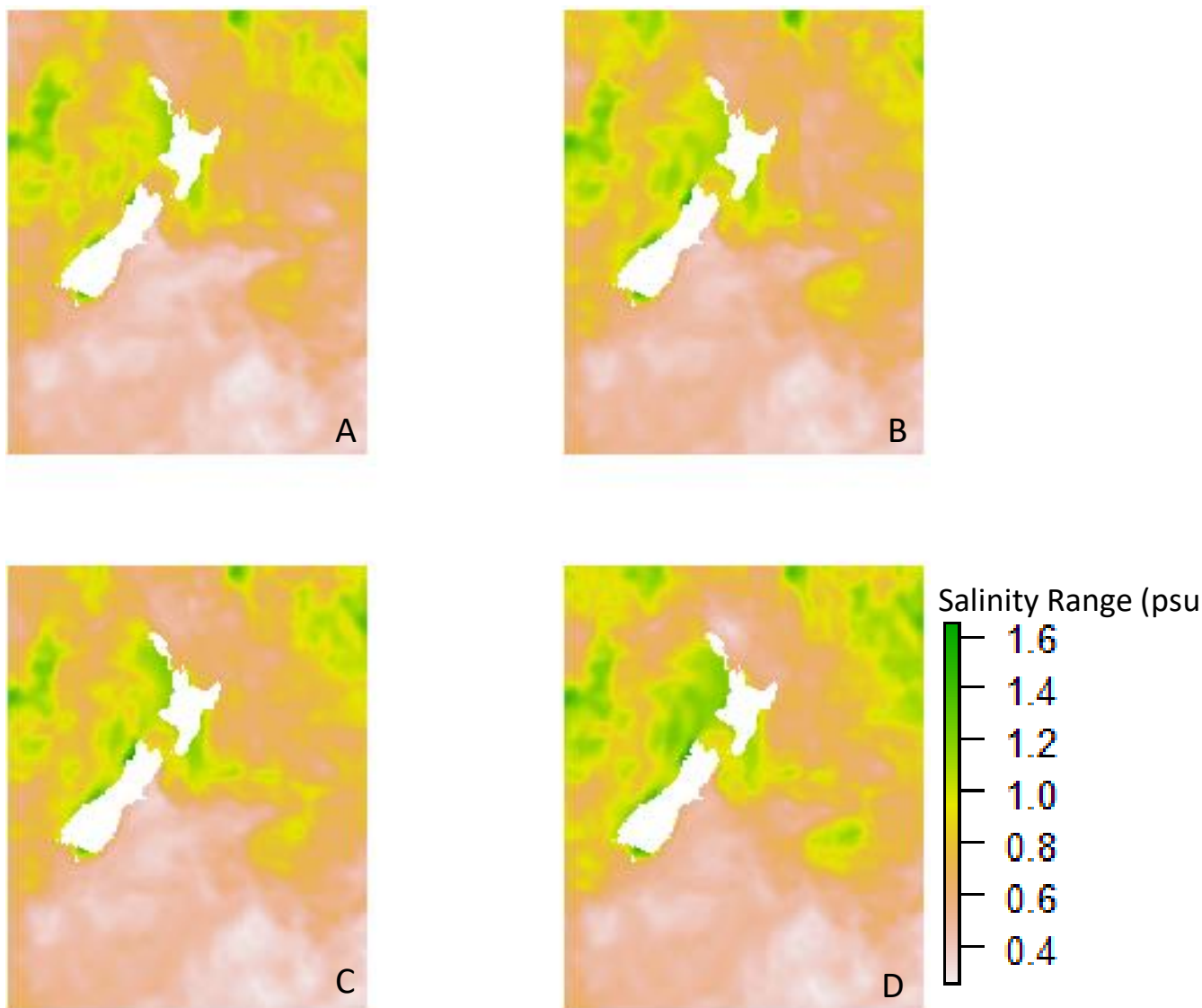

Appendix C.8: SS Range 2100 climate layers under RCP scenarios 26(A), 4.5(B), 6.0(C) and 8.5(D). 


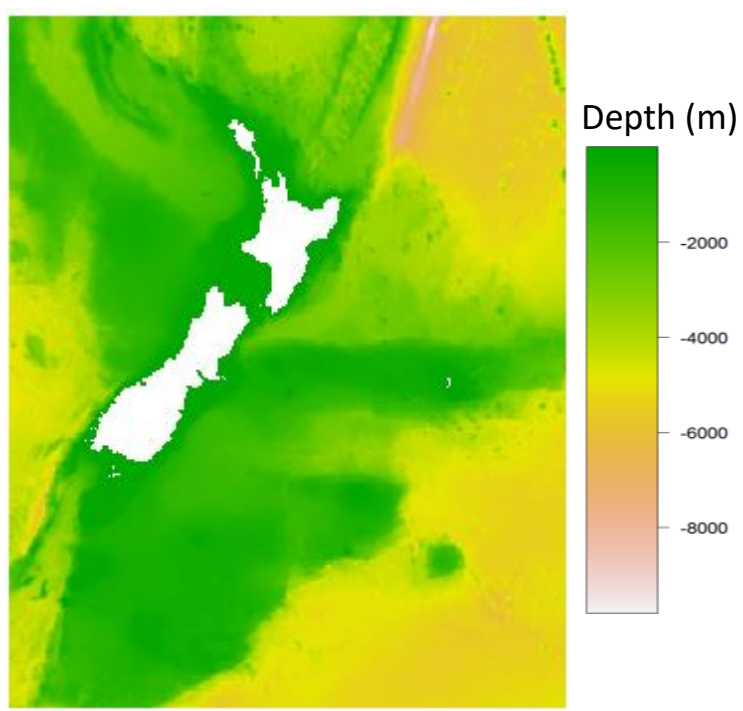

Appendix C.9: Bathymetric layer

Appendix D: Full sets of MESS maps for each species
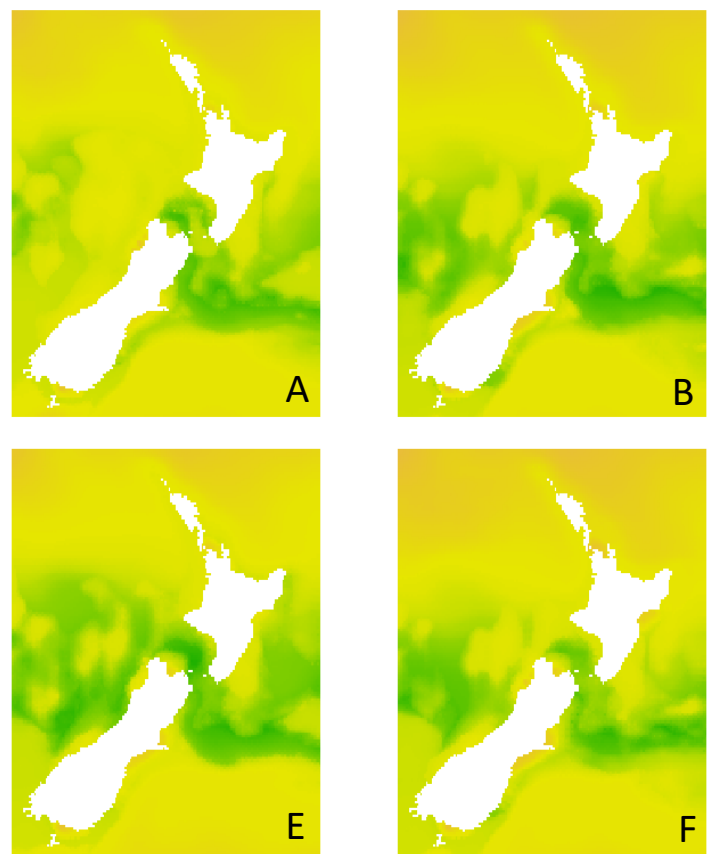
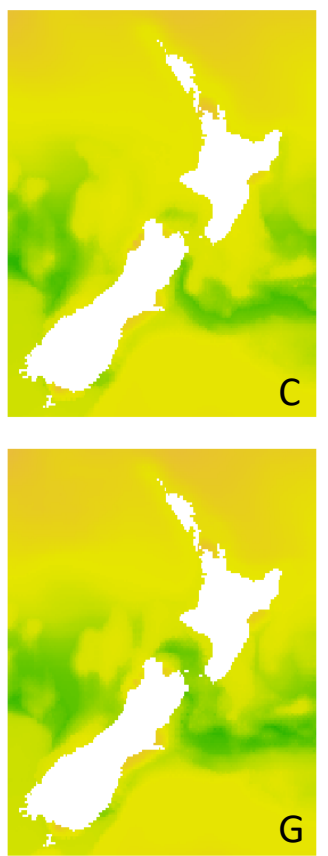

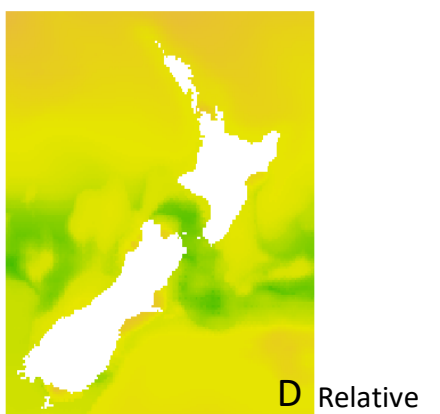

Uncertainty

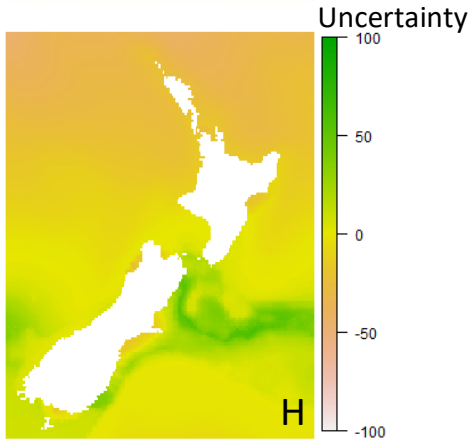

Appendix D.1: Snapper; MESS Maps of scenarios RCP2.6 (A, E), RCP4.5 (B, F), RCP65 (C, G), RCP8.5 (D, H) in 2050 (A-D), and 2100 (E-H). 

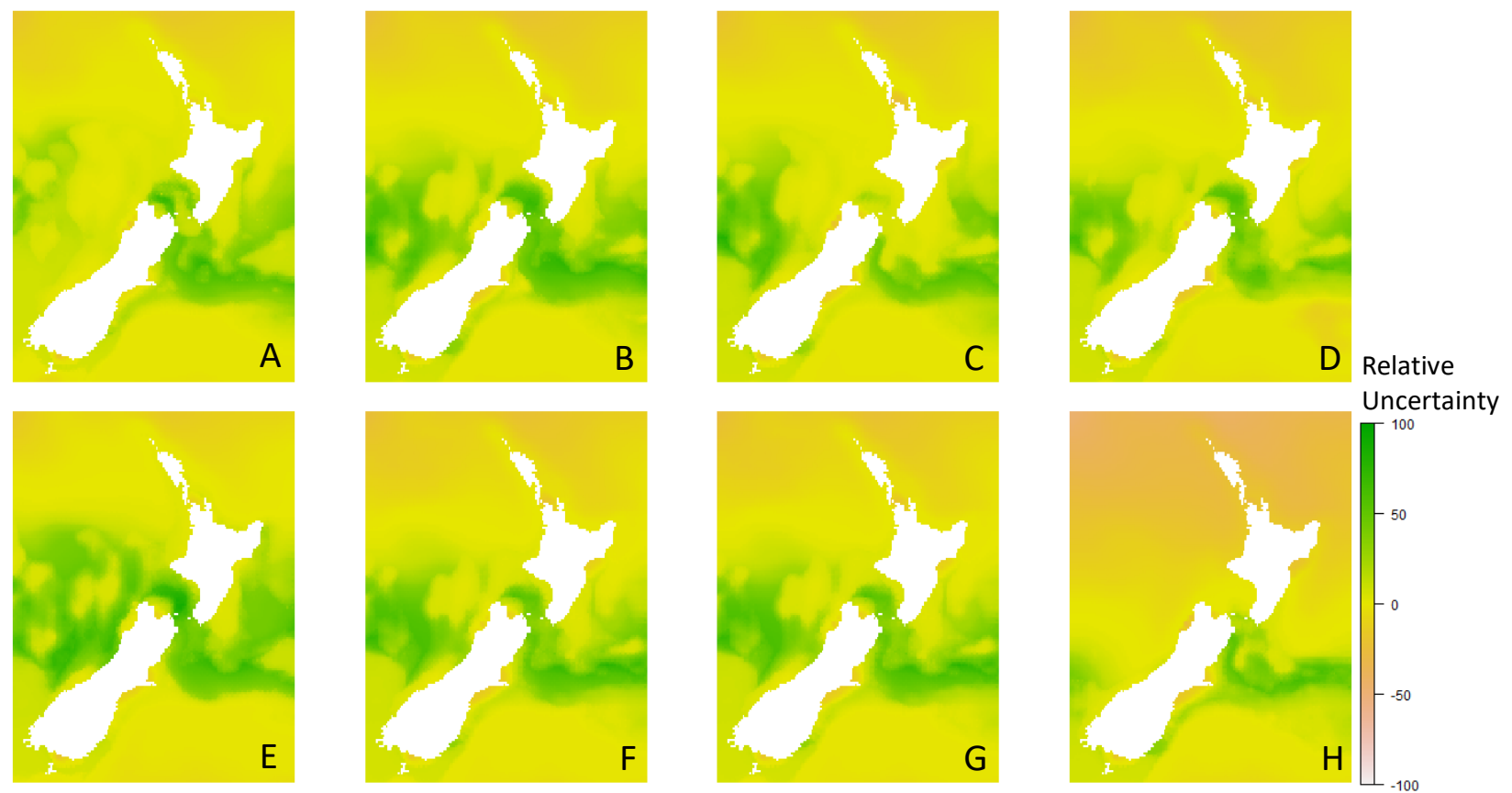

Appendix D.2: John dory; MESS Maps of scenarios RCP2.6 (A, E), RCP4.5 (B, F), RCP6.0 (C, G), RCP8.5 (D, H) in 2050 (A-D), and 2100 (E-H).
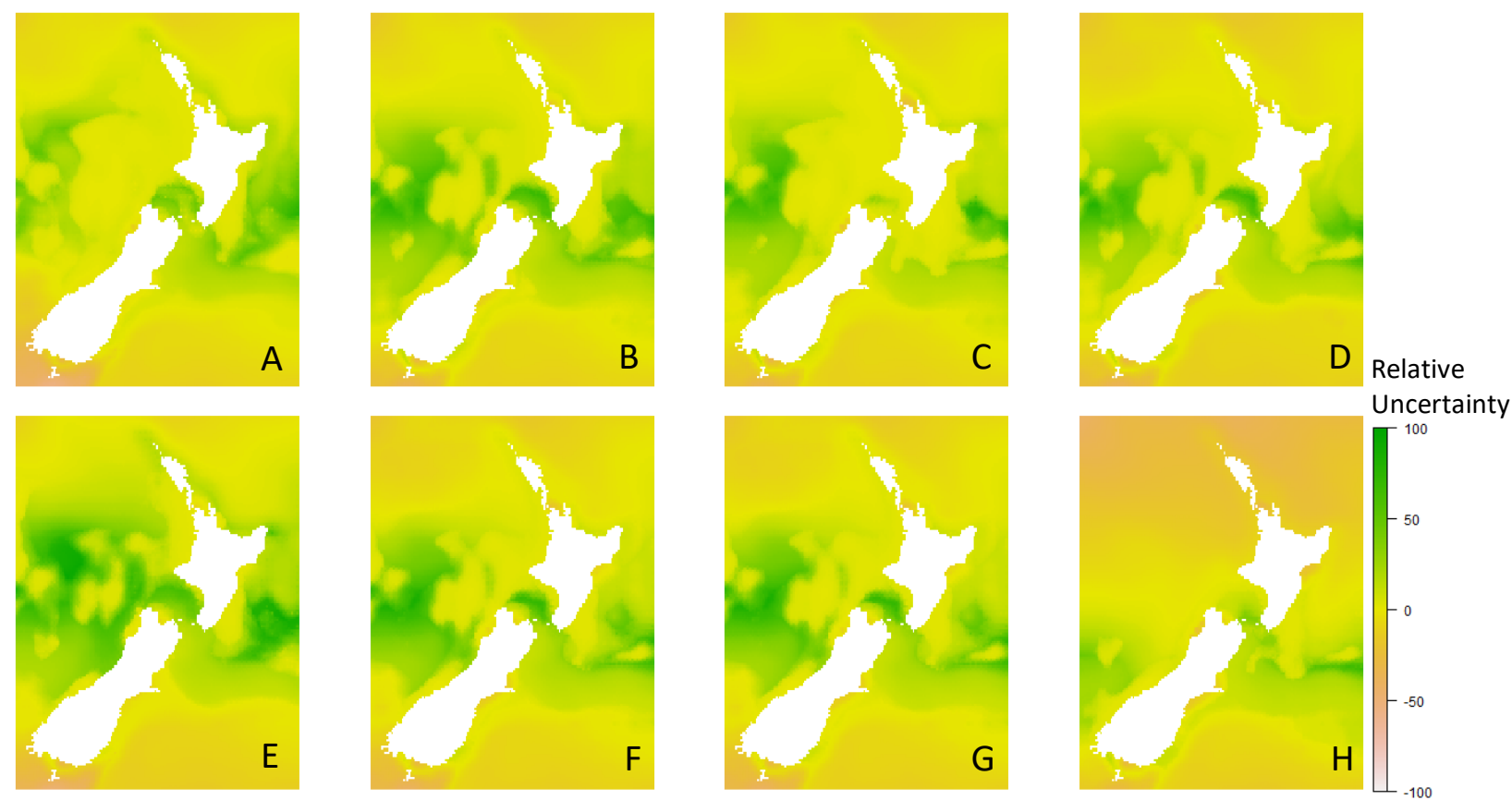

Appendix D.3: Trevally; MESS Maps of scenarios RCP2.6 (A, E), RCP4.5 (B, F), RCP6.0 (C, G), RCP8.5 (D, H) in 2050 (A-D), and 2100 (E-H). 

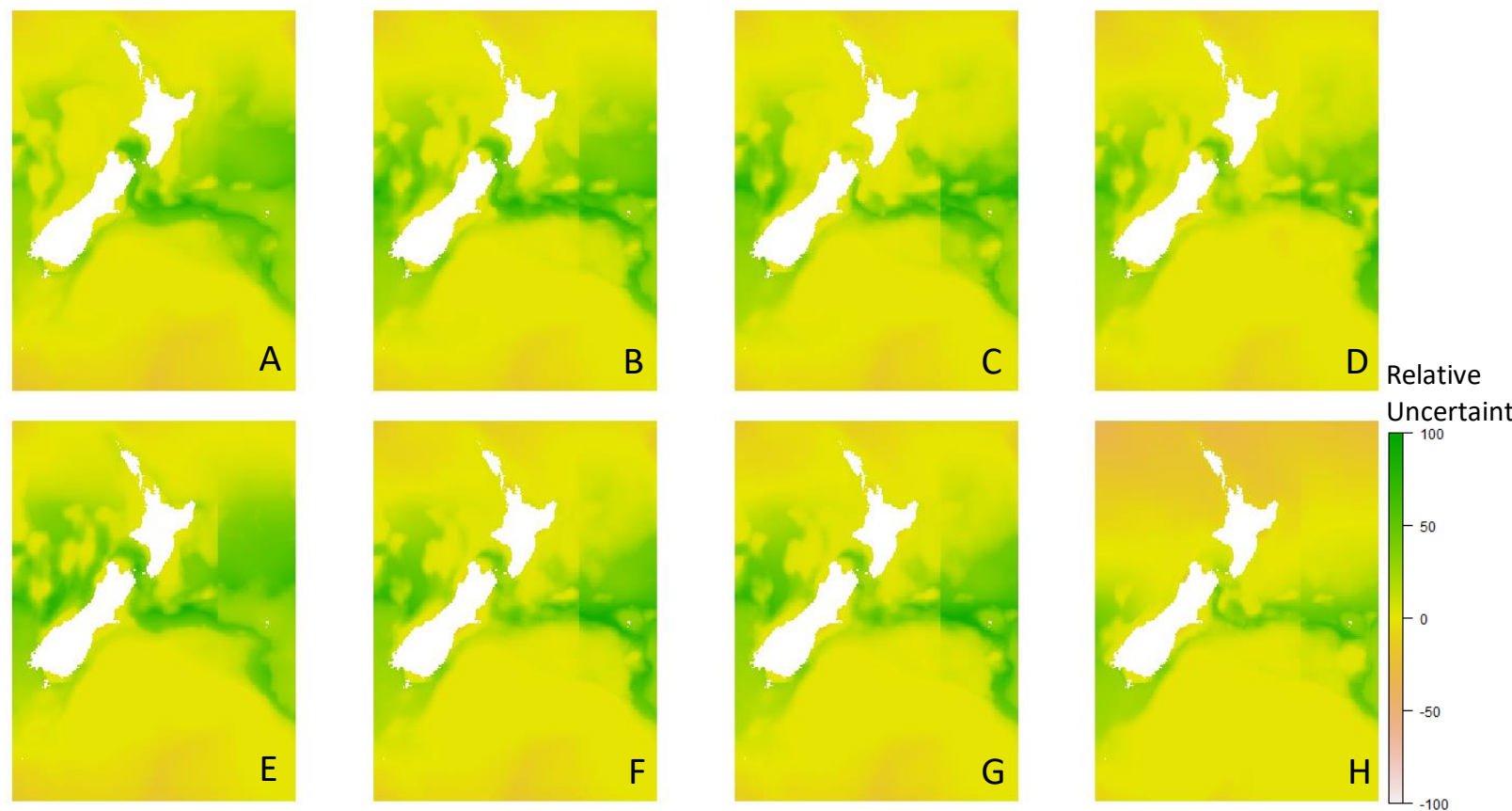

Appendix D.4: Tarakihi; MESS Maps of scenarios RCP2.6 (A, E), RCP4.5 (B, F), RCP6.0 (C, G), RCP8.5 (D, H) in 2050 (A-D), and 2100 (E-H).
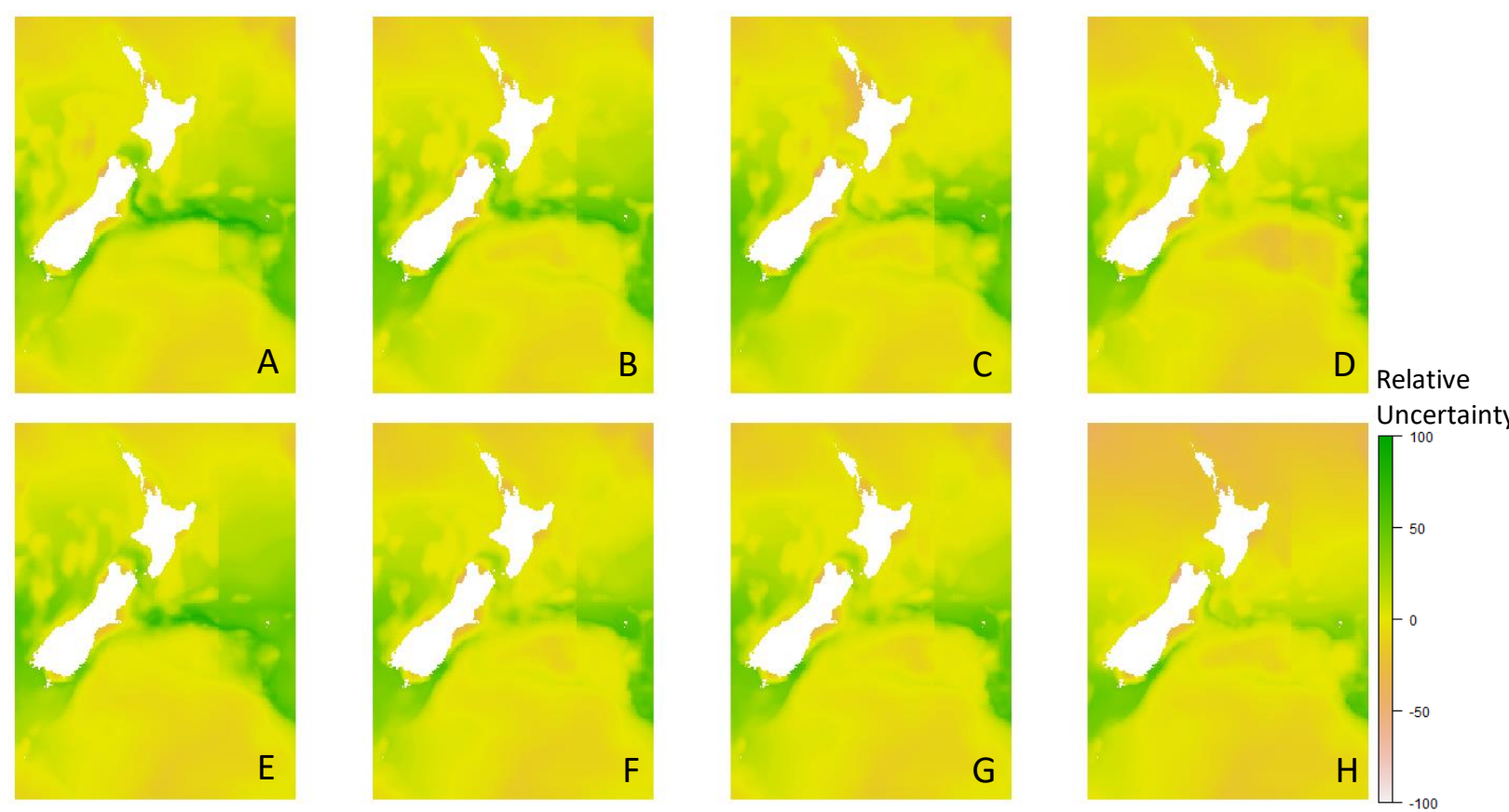

Appendix D.5: Scampi; MESS Maps of scenarios RCP2.6 (A, E), RCP4.5 (B, F), RCP6.0 (C, G), RCP8.5 (D, H) in 2050 (A-D), and 2100 (E-H). 

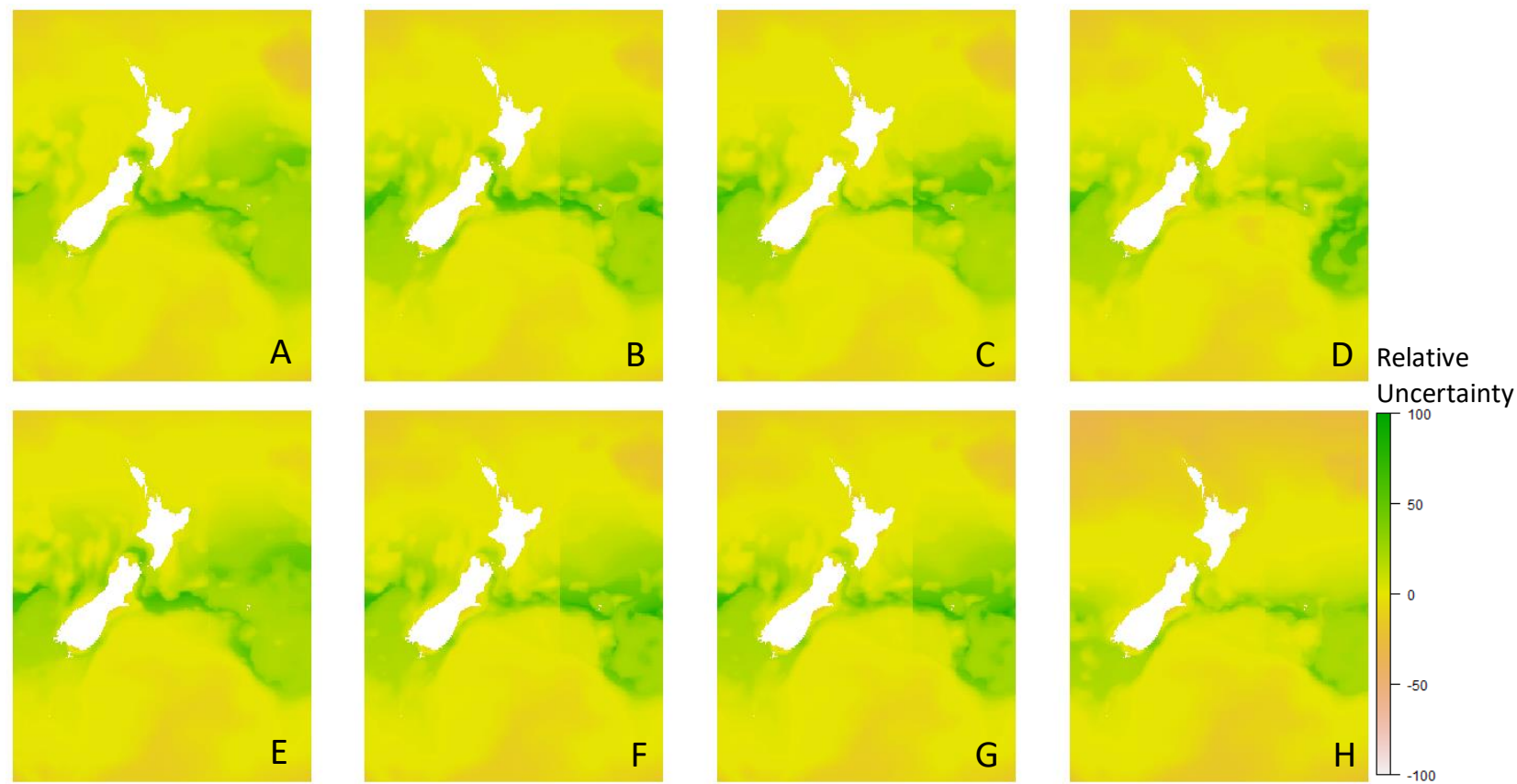

Appendix D.6: Orange roughy; MESS Maps of scenarios RCP2.6 (A, E), RCP4.5 (B, F), RCP6.0 (C, G), RCP8.5 (D, H) in 2050 (A-D), and 2100 (E-H).
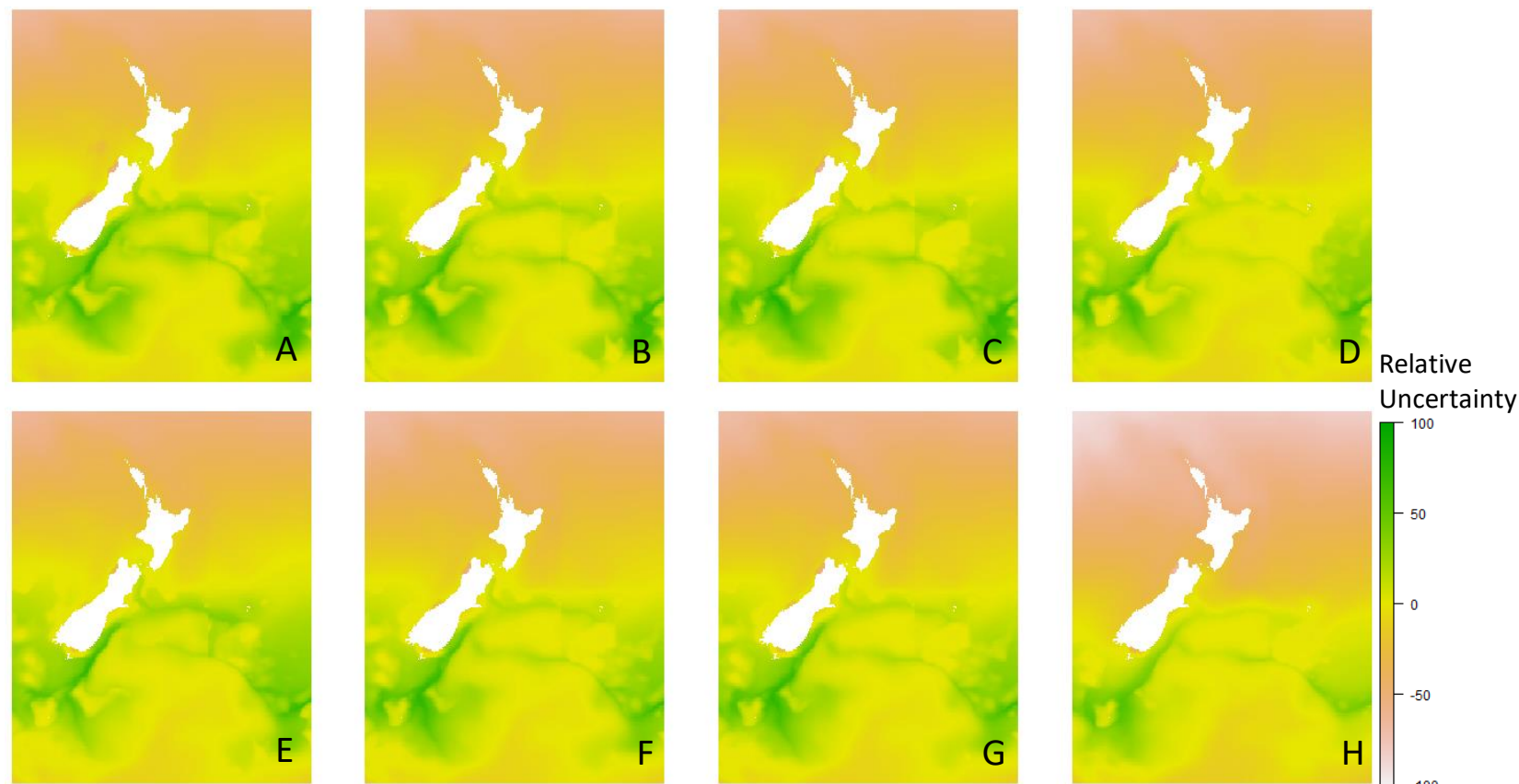
Uncertainty

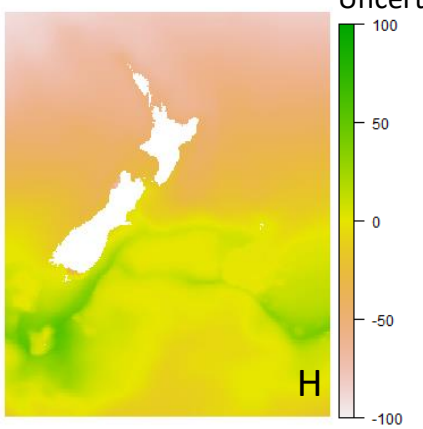

Appendix D.7: Southern blue whiting; MESS Maps of scenarios RCP2.6 (A, E), RCP4.5 (B, F), RCP6.0 (C, G), RCP8.5 (D, H) in 2050 (A-D), and $2100(E-H)$. 

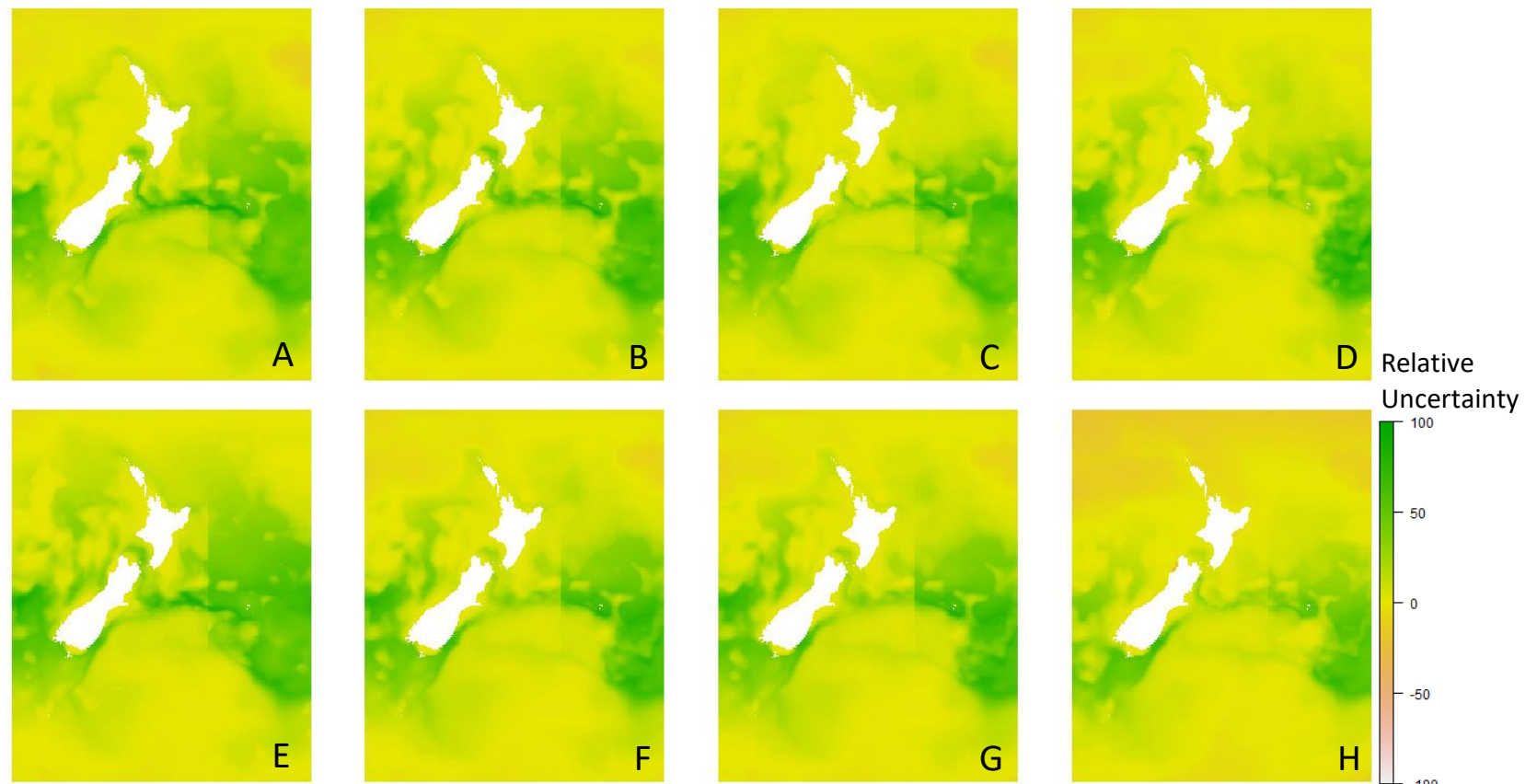

Appendix D.8: Hoki; MESS Maps of scenarios RCP2.6 (A, E), RCP4.5 (B, F), RCP6.0 (C, G), RCP8.5 (D, H) in 2050 (A-D), and $2100(E-H)$.
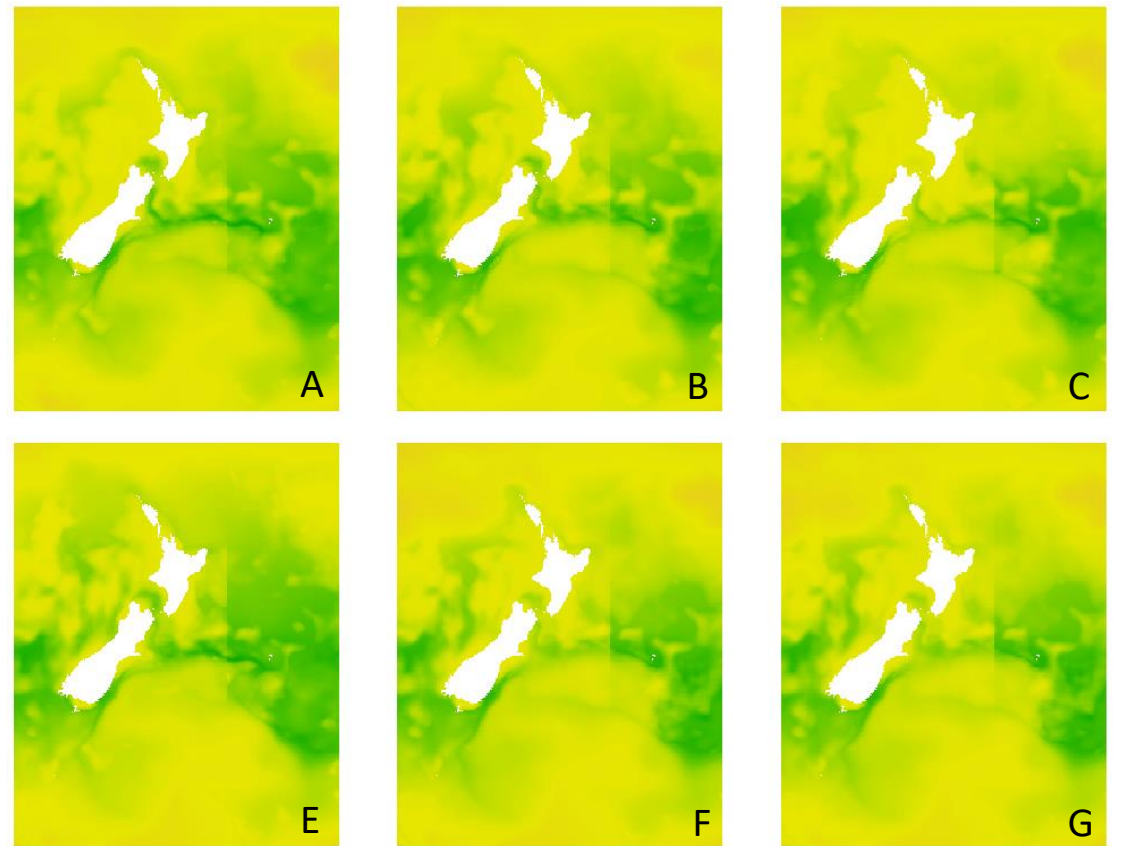

Appendix D.9: Ling; MESS Maps of scenarios RCP2.6 (A, E), RCP4.5 (B, F), RCP6.0 (C, G), RCP8.5 (D, H) in 2050 $(A-D)$, and $2100(E-H)$. 

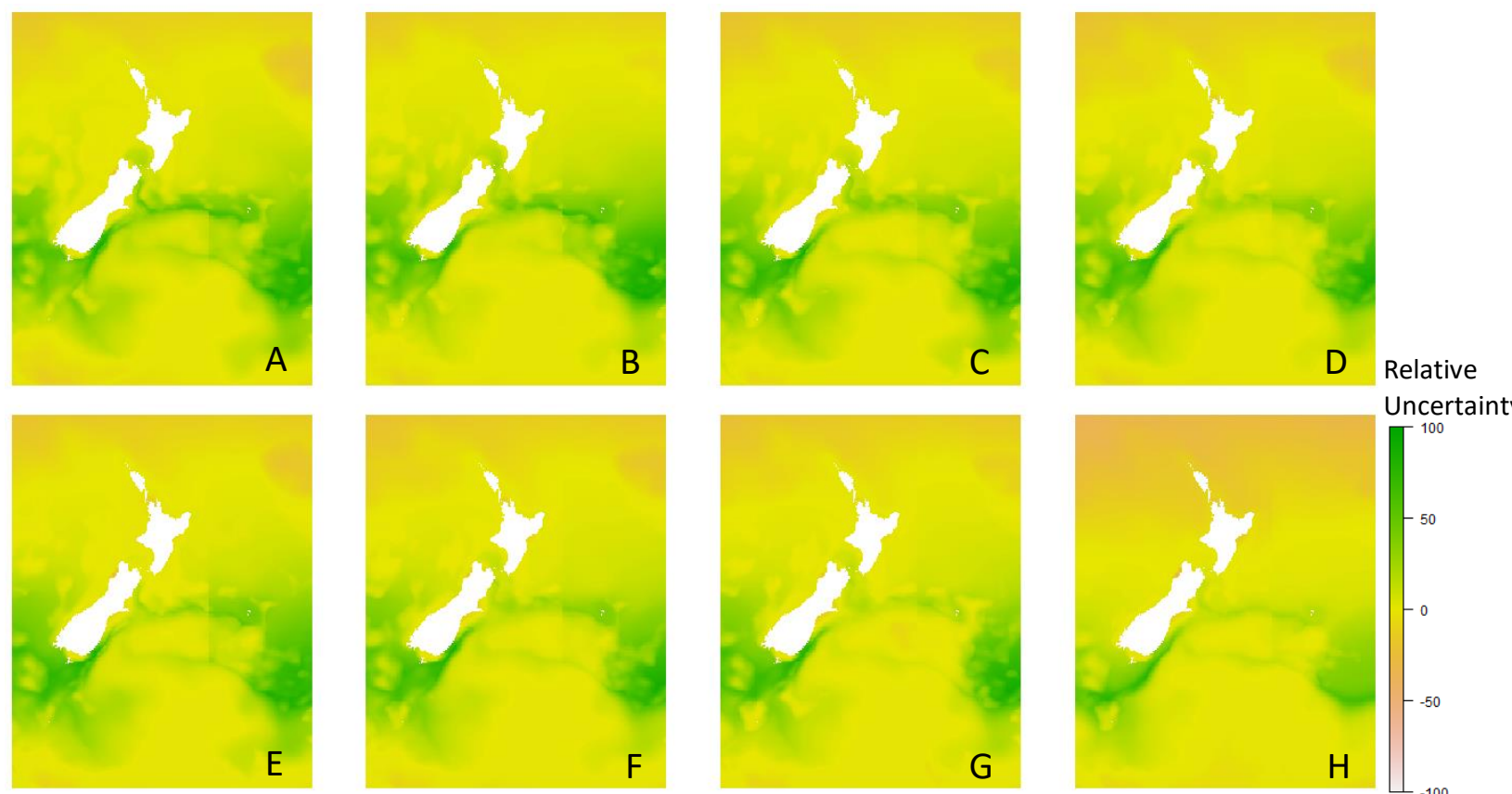
Uncertainty

Appendix D.10: Arrow squid; MESS Maps of scenarios RCP2.6 (A, E), RCP4.5 (B, F), RCP6.0 (C, G), RCP8.5 (D, H) in 2050 (A-D), and 2100 (E-H). 